\title{
Intracellular glycation and endothelial dysfunction : role of methylglyoxal
}

Citation for published version (APA):

Brouwers, O. (2011). Intracellular glycation and endothelial dysfunction : role of methylglyoxal. [Doctoral Thesis, Maastricht University]. Maastricht University. https://doi.org/10.26481/dis.20110518ob

Document status and date:

Published: 01/01/2011

DOI:

10.26481/dis.20110518ob

Document Version:

Publisher's PDF, also known as Version of record

\section{Please check the document version of this publication:}

- A submitted manuscript is the version of the article upon submission and before peer-review. There can be important differences between the submitted version and the official published version of record.

People interested in the research are advised to contact the author for the final version of the publication, or visit the DOI to the publisher's website.

- The final author version and the galley proof are versions of the publication after peer review.

- The final published version features the final layout of the paper including the volume, issue and page numbers.

Link to publication

\footnotetext{
General rights rights.

- You may freely distribute the URL identifying the publication in the public portal. please follow below link for the End User Agreement:

www.umlib.nl/taverne-license

Take down policy

If you believe that this document breaches copyright please contact us at:

repository@maastrichtuniversity.nl

providing details and we will investigate your claim.
}

Copyright and moral rights for the publications made accessible in the public portal are retained by the authors and/or other copyright owners and it is a condition of accessing publications that users recognise and abide by the legal requirements associated with these

- Users may download and print one copy of any publication from the public portal for the purpose of private study or research.

- You may not further distribute the material or use it for any profit-making activity or commercial gain

If the publication is distributed under the terms of Article $25 \mathrm{fa}$ of the Dutch Copyright Act, indicated by the "Taverne" license above, 


\section{INTRACELLULAR GLYCATION AND \\ ENDOTHELIAL DYSFUNCTION; ROLE OF METHYLGLYOXAL}


(C) Olaf Brouwers, Maastricht 2011

Cover design by Jean Scheijen | www.vierdrie.nl

Printed by GVO drukkers \& vormgevers B.V. | Ponsen \& Looijen

ISBN 978-90-6464-466-5 


\title{
INTRACELLULAR GLYCATION AND ENDOTHELIAL DYSFUNCTION; ROLE OF METHYLGLYOXAL
}

\author{
PROEFSCHRIFT \\ Ter verkrijging van de graad van doctor \\ aan de Universiteit Maastricht, \\ op gezag van de Rector Magnificus, \\ Prof. Mr. G.P.M.F. Mols \\ volgens het besluit van het College van Decanen, \\ in het openbaar te verdedigen \\ op woensdag 18 mei 2011 om 14.00 uur \\ door \\ Olaf Brouwers
}

Geboren te Heerlen op 12 juni 1982 


\section{Promotores:}

Prof. Dr. C.D.A. Stehouwer

Prof. Dr. J.G.R. De Mey

\section{Copromotor:}

Dr. C.G. Schalkwijk

\section{Beoordelingscommissie:}

Prof. Dr. H.A.J. Struijker-Boudier (voorzitter)

Prof. Dr. H.P. Hammes (University Hospital Mannheim - Mannheim)

Prof. Dr. V.W.M. van Hinsbergh (VUMC - Amsterdam)

Prof. Dr. N.C. Schaper

Prof. Dr. H.H.H. Schmidt

Financial support by the Netherlands Heart Foundation and Dutch Diabetes Research Foundation for the publication of this thesis is gratefully acknowledged.

Danish Myo Technology and Grace Davison Discovery Sciences are also gratefully acknowledged for their financial support. 


\section{CONTENTS}

$\begin{array}{lll}\text { Chapter } 1 & \text { General introduction } & 7\end{array}$

Chapter 2 Methylglyoxal and methylglyoxal-arginine adducts do not 37

directly inhibit endothelial nitric oxide synthase

Chapter 3 Hyperglycaemia-induced impaired endothelium- 45

dependent vasorelaxation in rat mesenteric arteries is

mediated by intracellular methylglyoxal levels in a

pathway dependent on oxidative stress

Chapter 4 Overexpression of glyoxalase-I reduces hyperglycaemia-

induced levels of advanced glycation endproducts and oxidative stress in diabetic rats

Chapter 5 Glyoxalase-I overexpression improves endothelial dysfunction and attenuates renal impairment in diabetic rats

Chapter 6 Glyoxalase-I overexpression improves blood pressure independently of cardiac function in a rat model of diabetes

Chapter 7 General discussion

Samenvatting

Dankwoord 147

Curriculum Vitae 151

List of publications 153

Abbreviations 155

Colour figures 157 

General introduction

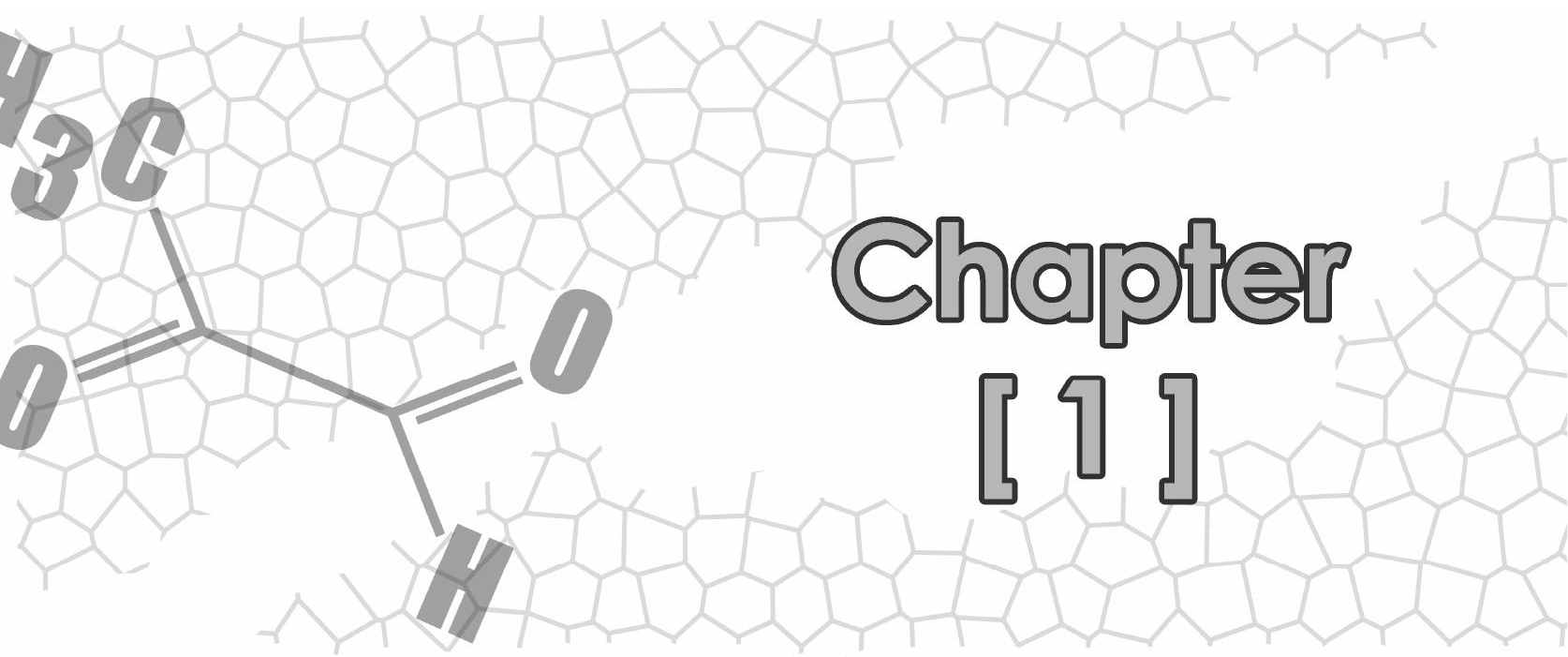




\section{Diabetes and the development of diabetic complications}

Diabetes mellitus is a chronic metabolic disorder characterised by a deficiency in the production (type 1 diabetes; T1DM) or the effect of insulin (type 2 diabetes; T2DM). As a result, this defect increases blood glucose levels, which consequently may damage the vasculature and important target organs, such as the retina, the kidneys, the heart and the nervous system (1). Worldwide 171 million people have diabetes and the largest and fastest growing group, consisting of approximately 90\%, have T2DM. Mainly due to the age- and obesity-related increase in T2DM, the total number of people with diabetes is believed to increase to approximately 366 million people in 2025 (2). Both types of diabetes are characterised by chronic hyperglycaemia and prolonged exposure to hyperglycaemia is the primary causal factor in the development of diabetes-related complications. Diabetic complications can be roughly divided in two major categories, namely micro- and macrovascular complications. These complications are the principal course of the high morbidity and mortality in patients with diabetes (3; 4). Macrovascular diseases affect the larger arteries which supply the heart, brain and lower extremities. Therefore, diabetic individuals have a much higher risk for myocardial infarction, stroke and limb amputation (5). The damage to the small arteries is believed to be of even more importance, because the effects on blood flow and vascular permeability already occur very early in the course of diabetes. Retinal and renal microangiopathy cause diabetic retinopathy and nephropathy, and damage to the vasa nervorum is important in the development of diabetic neuropathy (6-8).

\section{Molecular mechanisms underlying hyperglycaemia-induced damage}

Cardiovascular diseases are multifactorial and besides genetic factors also a non-healthy diet, lack of exercise and habits like high alcohol consumption and smoking increase the risk on developing cardiovascular problems (9). Diabetic individuals have a four-fold increased risk of developing cardiovascular complications. Various mechanisms have been described to explain how hyperglycaemia directly causes vascular complications. These pathways are: increased flux through the polyol pathway, activation of the protein kinase $C$ (PKC) pathway, increased hexosamine pathway (HSP) flux and increased formation of advanced glycation endproducts (AGE) (see figure 1.1). The polyol pathway, PKC pathway and the HSP pathway will be briefly described. The AGE pathway is the focus of this thesis and will be described in more detail.

\section{The polyol pathway}

The enzyme aldose reductase is the first enzyme in the polyol pathway, in which glucose and other carbonyl-containing compounds are reduced to sorbitol. Aldose reductase is 
dependent on nicotinamide adenine dinucleotide phosphate (NADPH), and in circumstances of hyperglycaemia the affinity of the enzyme for glucose increases and an excess of NADPH is used in this pathway. Subsequently sorbitol is oxidised to fructose, which increases the NADH:NAD ${ }^{+}$ratio.

The first proposed mechanism by which the polyol pathway could exercise its detrimental effects was by sorbitol-induced osmotic damage to microvascular cells. However other pathways are more likely to be involved, because the sorbitol levels measured in diabetic vessels are probably too low to cause osmotic damage. The decrease in NADPH, due to overactivity of the polyol pathway, can lead to a deficiency in reduced glutathione (GSH) (11). Furthermore, the decrease in $\mathrm{NAD}^{+}$, by the increase in sorbitol oxidation, inhibits the activity of the enzyme glyceraldehyde-3-phosphate dehydrogenase (GAPDH), which results in increasing amounts of reactive triose phosphates (12). As a result, both pathways increase oxidative stress and the formation of AGEs (which will be discussed later). Pharmacological inhibition of the aldose reductase enzyme has been shown to give significant benefit against the development of various diabetic complications in both animal models (13-15) and diabetic subjects (16-18).

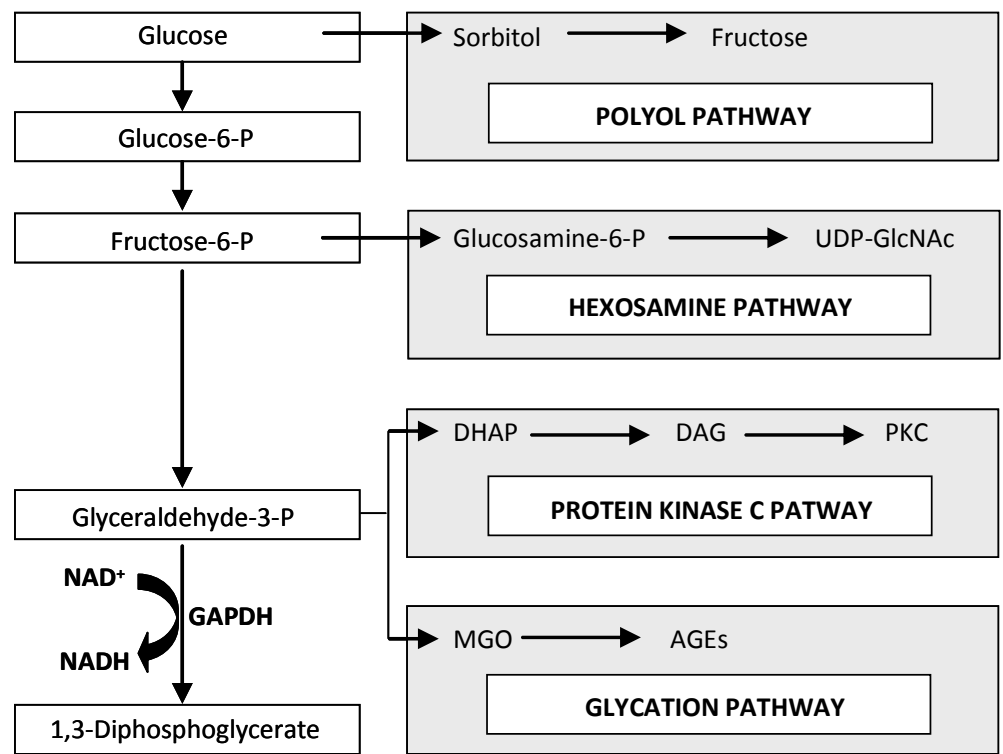

Figure 1.1. Molecular mechanisms underlying hyperglycaemia-induced damage

Increased glucose levels activate four different pathways which contribute to hyperglycaemic-induced damage. These pathways include increased flux through the polyol pathway, increased modification of proteins by $\mathrm{O}$ linked $\mathrm{N}$-acetylglucosamine via the hexosamine pathway, activation of protein kinase $\mathrm{C}$ via diacylglycerol, and formation of advanced glycation endproducts via the main intracellular precursor methylglyoxal (figure modified from Brownlee (10)). 


\section{The protein kinase $\mathrm{C}$ pathway}

PKC is a large family of serine-threonine kinases with closely related structures but different properties. Activation of PKC occurs through increased levels of the PKC activator diacylglycerol (DAG). DAG is synthesised from the glycolytic intermediates dihydroxyaceton phosphate (DHAP) and glyceraldehyde-3-phosphate (G3P) (19). Several studies have suggested the importance of the activation of specific PKC isoforms, mainly the $\alpha, \beta 1 / 2$ and $\delta$ isoforms, in causing cardiac or microvascular pathologies in diabetic animals $(20 ; 21)$. PKC activation results in downregulation of endothelial nitric oxide synthase (eNOS), as well as a variety of other deleterious vascular events like abnormal coagulation, enhanced production of the vasoconstrictor endothelin-1, and an increase in vascular permeability $(22 ; 23)$.

Due to the large variety of PKC subtypes, and the only few available selective PKC isoform inhibitors, the exact contribution of the specific PKC isoforms in hyperglycaemia-induced damage is still largely unknown. However, the use of a specific PKC $\beta$ inhibitor prevented accelerated glomerular mesangial expansion in a mouse model of T2DM (24).

\section{The hexosamine pathway}

The first and rate-limiting enzyme in the HSP is glutamine:fructose-6-phosphate aminotransferase that utilizes glutamine and fructose-6-phosphate for the formation of glucosamine-6-phosphate and glutamate. Following subsequent enzymatic reactions, the pathway eventually leads to the formation of uridine diphosphate $\mathrm{N}$-acetylglucosamine (UDP-GICNAc). This nucleotide sugar can be either converted into other types of nucleotide sugars or be directly incorporated into a variety of glycosyl-containing macromolecules, via a process which resembles phosphorylation (25). Two enzymes carry out the cycling of UDP-GICNAc; O-linked N-acetylglucosamine transferases, which catalyzes the O-linkage to serine and threonine residues and meningioma expressed antigen 5 -hyaluronidase which removes the hexosamine moiety $(26 ; 27)$. In addition to serving as a precursor for a diverse set of glycoconjugates, UDP-GIcNAc also inhibits glutamine:fructose-6-phosphate aminotransferase activity through a negative feedback mechanism to reduce the flux through the HSP (28).

Increased flux through the hexosamine pathway, due to hyperglycaemia, impairs activation of the insulin / protein kinase B pathway, resulting in dysregulation of eNOS activity (29). Hyperglycaemia-associated inhibition of eNOS is induced by a two-fold increase in GIcNAcylation of eNOS with a reciprocal decrease in phosphorylation at Serine1177 (30). Consistent with this, carotid plaques from diabetic patients showed increased GlcNAcylation in endothelial cells compared to non-diabetic patients, with a six-fold increase in O-GIcNAc modification of the cap plaques (29). High glucose, but also hyperinsulinaemia, increases the GlcNAcylation of many proteins in myotubes including 
heat-shock protein 70, $\alpha$-tubulin, and the transcription factor sp1 (31). Sp1 contains multiple O-GIcNAc residues and GlcNAcylation of sp1 activates the expression of several genes associated with diabetes, probably by blocking sp1 interaction with suppressor proteins (32).

\section{Advanced glycation endproduct formation}

The nonenzymatic reaction of an amino group with a reducing sugar, resulting in the formation of complex brown pigments and protein-protein crosslinks, was first described in the early 1900 s by Louis-Camille Maillard (33). The reaction, which became known as the Maillard reaction, was soon recognized by food chemists as explanation for brown colour formation and loss of protein quality in cooked and stored food. However, since the discovery that the haemoglobin glycosylated isoform $\mathrm{HbA} 1 \mathrm{c}$ is present in subjects with diabetes, most interest was shifted to Maillard-like reactions in vivo (34). Throughout the 1980s and 1990s, a large body of evidence has accumulated that the stable endproducts of the Maillard reaction, the AGEs, are mediators of various complications in diabetes and aging (35-37). The formation of these AGEs can originate from various pathways. In addition to the classical Maillard reaction, AGEs can also be formed intracellularly from glucose- or lipid-derived dicarbonyl precursors (see figure 1.2).

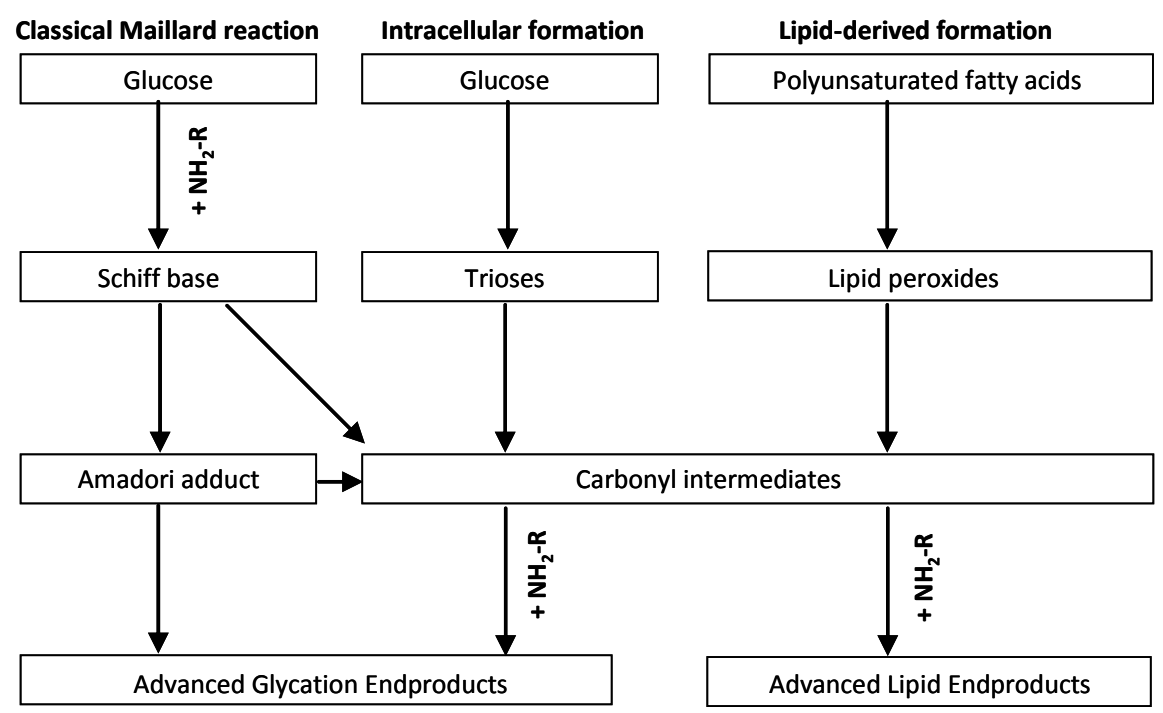

Figure 1.2. The three major pathways of advanced glycation endproduct formation

AGEs are endogenously formed mainly by three different pathways: the classical Maillard pathway, intracellular AGE formation, and the AGE formation which originates from lipids. 
The Maillard reaction is subdivided into three main stages; the early, intermediate and late stage. In the early stage of the Maillard reaction, a carbonyl group (aldehyde or keton) of glucose (or other reduced sugars such as fructose and pentose) reacts with free amino groups of proteins, but also nucleic acids and lipids, to form a Schiff base. In the intermediate stage, rearrangements of these Schiff bases gives rise to a stable ketoamine, known as the Amadori product. Finally, in the late stage these Amadori products undergo further rearrangement, dehydration, and condensation to from irreversible AGEs (38). Because of their slow formation, it was long believed that AGEs accumulate exclusively on long-lived extracellular proteins, such as skin collagen and lens proteins. Baynes et al. showed that AGE accumulation on these proteins was strongly associated with age and was increased in diabetes (39).

In addition to the classical pathway of AGE formation, a more rapid intracellular AGE formation on short-lived proteins is believed to be of even more importance in vivo. Glucose has a very slow glycation rate. In contrast, glucose-derived glycolytic intermediates or intracellular sugars form not only many more glycated proteins, but also more rapidly. The glucose-derived glycolytic intermediates glucose-6-phosphate, G3P, DHAP, and the dicarbonyl compounds glyoxal (GO), methylglyoxal (MGO) and 3deoxyglucosone (3-DG) play an important role in this fast intracellular Maillard reaction (40). Furthermore, the sorbitol pathway generates reactive intermediates such as fructose, fructose-3-phosphate and 3-DG, and these intermediates may also react with proteins and substantially contribute to fast intracellular AGE formation. Therefore, it seems likely that intracellular hyperglycaemia is the primary initiating event in the formation of AGEs and also contributes to the pool of circulating AGEs (10).

The third major route of AGE formation originates from lipids. Lipid peroxidation of polyunsaturated fatty acids leads first to the formation of lipid peroxides, which then decompose in a large variety of reactive carbonyl compounds (41). Some of them are identical to those formed from glucose-derived glycolytic intermediates, such as GO and MGO, and others are characteristic lipid peroxidation products, such as malondialdehyde (MDA) and hydroxynonenal (HNE) (42). These reactive intermediates then further react with cysteine, histidine and lysine residues, resulting in glycated proteins. The resulting lipid-derived modifications are called advanced lipid endproducts.

\section{Oxidative stress as a common pathway in hyperglycaemia-induced damage}

Many studies have shown that, besides activation of the four previously described pathways, diabetes also increases the production of reactive oxygen species (ROS) (43). The observation that inhibition of the overproduction of ROS by the mitochondrial electron-transport chain also decreases the activity of the polyol, PKC and hexosamine pathways and prevents the formation of AGEs indicates that oxidative stress is a common 
link in all these four pathways $(32 ; 44)$. In this regard, an important effect of the hyperglycaemia-induced overproduction of mitochondrial superoxide is the prominent decrease in GAPDH activity. The nuclear enzymes poly(ADP-ribose) polymerases (PARP enzymes), play an important role therein. This DNA repair enzyme can, upon overactivation by extensive nitrosative and oxidative stress, modify GAPDH, thereby decreasing its activity and promoting accumulation of glycolytic metabolites upstream of GAPDH (45). In this paragraph the formation of ROS will be discussed and also the main mechanisms of action of hyperglycaemia-induced ROS.

Oxidative stress results from either an excessive production of ROS, loss of antioxidant defences, or both (46). The major sources of ROS are either mitochondrial or cytosolic. Mitochondria contain five main enzymatic and electron transporting complexes: complex-I (NADH dehydrogenase), complex-II (succinate dehydrogenase), complex-III (ubiquinone cytochrome c oxidoreductase), complex-IV (cytochrome oxidase) and complex V (F1FOATP synthase). The specific dehydrogenases use the energy electrons derived from glucose or free fatty acids metabolism to catalyze the conversion of ADP to ATP by ATP synthase. However not every energy electron is used for ATP synthesis. During passages through the electron chain, energy is gradually released from electrons, but also transferred to $\mathrm{O}_{2}$, thereby generating ROS. The principal mitochondrial sites of oxidative stress generation are complex I and III (47). The mitochondrial protein gradient is partly modulated by uncoupling proteins (UCPs), which are localized into the inner-membrane and function as protein carriers, which bypass ATP synthesis and can use energy to sustain thermogenesis. Upregulation of UCPs by oxidative stress indicates that this mechanism is important in the regulatory decrease of mitochondrial ROS production (48).

In the cytosol, NAD(P)H oxidases catalyze the transfer of electrons from NADPH to molecular oxygen via their catalytic subunits to generate superoxide $\left(\mathrm{O}_{2}{ }^{-}\right)$and, as shown in some studies, hydrogen peroxide $\left(\mathrm{H}_{2} \mathrm{O}_{2}\right)$. Another important cytosolic source of ROS, especially in the vasculature, is uncoupled eNOS. When increased levels of $\mathrm{O}_{2}{ }^{-}$react with nitric oxide (NO), peroxynitrite $\left(\mathrm{ONOO}^{-}\right)$is formed. This ROS can consequently oxidize tetrahydrobiopterin $\left(\mathrm{H}_{4} \mathrm{~B}\right.$, a cofactor for eNOS), resulting in the dysfunction of eNOS and production of even more $\mathrm{O}_{2}{ }^{-}$instead of $\mathrm{NO}$ (49).

To neutralize these ROS, all cells require antioxidant enzymes. The most important antioxidant enzymes in the human body are superoxide dismutase (SOD), catalase, and glutathione peroxidase.

SODs are metal-containing enzymes that depend on a bound manganese, copper or zinc for their antioxidant activity. In mammals, the manganese-containing enzyme (MnSOD) is most abundant in mitochondria, while the zinc or copper forms (Cu/Zn SOD) are predominant in cytosol (50). $\mathrm{O}_{2}^{-}$is enzymatically degraded to $\mathrm{H}_{2} \mathrm{O}_{2}$ by SOD. Catalase is the major enzyme to remove $\mathrm{H}_{2} \mathrm{O}_{2}(51)$. It is found in peroxisomes of eukaryotic cells. Catalase 
degrades $\mathrm{H}_{2} \mathrm{O}_{2}$ to water and oxygen, and hence finishes the detoxification reaction started by SOD. In addition, also glutathione peroxidase converts $\mathrm{H}_{2} \mathrm{O}_{2}$ to water with the addition of GSH, which is catalyzed to oxidized glutathione (GSSG). In addition to these enzymes, some compounds like vitamin $\mathrm{E}$, vitamin $\mathrm{C}$ and uric acid may participate in the elimination of oxygen radicals (52).

In diabetes, hyperglycaemia-induced ROS in endothelial cells overwhelms the cellular antioxidant capacity and impairs cellular defences. Hyperglycaemia results in an overdrive of the electron-transport chain, resulting in overproduction of superoxide anions. One of the main effects of ROS in endothelial cells is an impaired endothelium-dependent vasorelaxation. Besides the induction of apoptosis and therefore loss of endothelial cells, ROS can also inactivate $\mathrm{NO}$ via the production of $\mathrm{ONOO}^{-}(53 ; 54)$. Formation of $\mathrm{ONOO}^{-}$is also referred to as nitrosative stress which can alter structures of proteins (55). Besides this, ROS also induces upregulation of adhesion molecules (56), disruption of intercellular junctions (57), and plasminogen activator inhibitor-1 (PAI-1) release (58), thereby promoting vascular dysfunction.

Oxidative stress activates the four molecular mechanism of hyperglycaemia-induced damage, but these pathways can also be an underlying mechanism contributing to the detrimental effects of ROS. Activation of the polyol pathway enhances the oxidative stress response by depleting endogenous GSH levels (59), an important source of antioxidant capacity, and by inhibiting GAPDH activity (12). The PKC pathway has been linked to oxidative stress generation by activating NADPH oxidases (60) and in the hexosamine pathway glucosamine is known to increase $\mathrm{H}_{2} \mathrm{O}_{2}$ levels (61). Finally the AGE pathway is known to trigger receptor-mediated processes and protein modification which can generate ROS (62-64).

The glycolic intermediates MGO, GO and 3-DG can be formed in all stages of the glycation process, and are also known to induce oxidative stress (40). The focus in this thesis is mainly on the involvement of the most reactive AGE precursor MGO in the development of vascular complications (65).

\section{The major advanced glycation endproduct precursor methylglyoxal}

MGO is a reactive $\alpha$-oxoaldehyde and a physiological metabolite of glucose. It is a small molecule with a molecular weight of $72 \mathrm{Da}$ and contains a ketone group and an aldehyde moiety of which the aldehyde group is more reactive (66). It has three forms in an aqueous solution: unhydrated (1\%), monohydrate $(71 \%)$ and dihydrate $(28 \%)$, which are in rapid equilibrium (67). MGO can be formed from various pathways, modifies amino acids and is detoxified by several enzymatic pathways (see figure 1.3). 


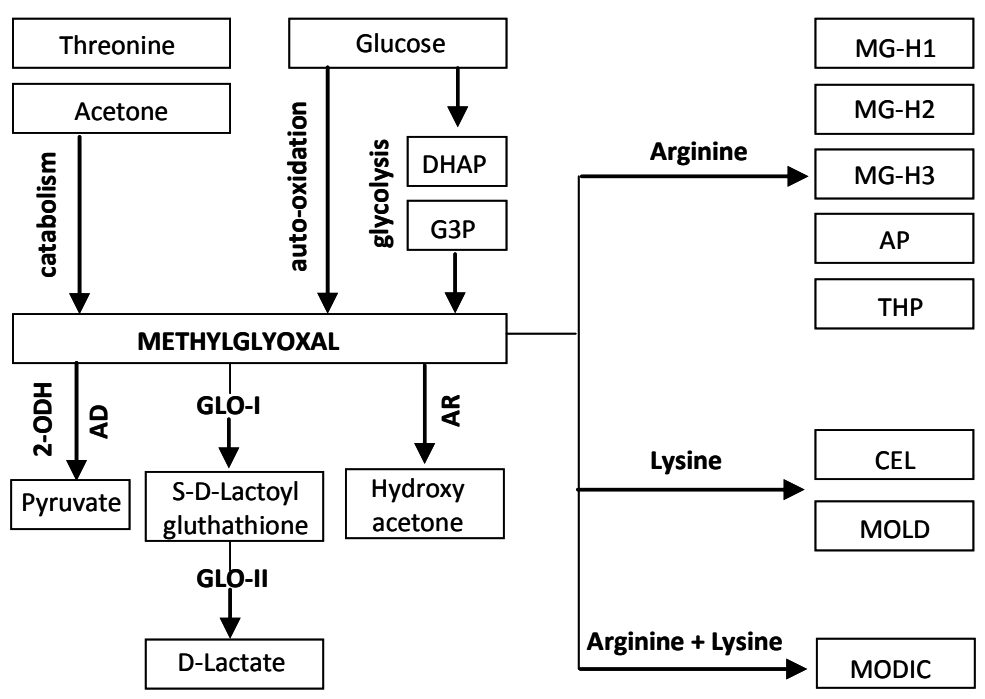

Figure 1.3. Formation, detoxification, glycation of/by methylglyoxal

MGO is mainly formed as a by-product of glycolysis and from auto-oxidation of glucose. Other sources of MGO formation are from the catabolism of threonine and acetone. Increased levels of MGO can be detoxified by predominantly the glyoxalase (GLO) pathway. Also aldose reductase (AR) decreases levels of MGO; aldehyde dehydrogenase $(A D)$ and 2-oxoaldehyde dehydrogenase (2-ODH) are minor pathways of detoxification. If production exceeds its elimination, MGO can modify arginine residues to form the AGEs, MG-H1, MG-H2, MGH3, AP and THP. With lysine MGO reacts to CEL and the lysine dimer MOLD. MGO can also form a dimer crosslink with arginine and lysine: MODIC.

\section{Major and minor pathways of methylglyoxal formation}

Endogenous MGO is formed from metabolic intermediates of carbohydrates, proteins and fatty acids. The majority of MGO is derived from the metabolites of carbohydrate, such as glucose and fructose. It can be formed non-enzymatically from G3P and DHAP (68; 69), and enzymatically from DHAP by MGO synthase in some mammalian tissues (70). In addition triosephosphate isomerase hydrolyses G3P and DHAP and removes phosphate to yield MGO (71). A minor source of $M G O$ is from aminoacetone during catabolism of threonine and from hydroxyacetone in the metabolism of acetone $(72 ; 73)$. These pathways are especially important in ketosis and diabetic acidosis. It has also been suggested that MGO can be formed via the process of lipid peroxidation (74).

\section{Enzymatic detoxification of methylglyoxal}

The rate of MGO formation is approximately $120 \mu \mathrm{M} /$ day or $0.1 \%$ of the glycolytic flux under normoglycaemic conditions $(68 ; 75)$. However, this small fraction of glucotriose flux is important because of the high reactivity of MGO with nucleic acids and proteins. Detrimental effects of MGO are usually avoided by enzymatic detoxification. 


\section{Major pathways of methylglyoxal detoxification}

The most important enzymatic MGO detoxification pathway is the glyoxalase pathway, in which D-lactate is the end-product. During the first step in this pathway a hemithioacetal is formed non-enzymatically by the reaction of MGO with GSH. Consequently, the rate limiting enzyme glyoxalase-I (GLO-I) facilitates the formation of S-D-lactoylglutathione. Finally glyoxalase-II catalyses the hydrolysis of S-D-lactoylglutathione to D-lactate, thereby reforming the GSH (76). Also GO is detoxified by the glyoxalase system, resulting in the formation of S-glycolylglutathione and consequently glycolate as the endproduct (77).

Van der Jagt et al. described that the enzyme aldose reductase is able to convert MGO to hydroxyacetone (78). However, tissue MGO levels can also be increased by the influence of aldose reductase enzyme activity. Aldose reductase catalyzes the NADPH-dependent reduction of MGO into lactaldehyde in the presence of GSH and then to 1,2-propanediol. However, acetol is formed during the first step, when GSH is absent. Therefore, GSH depletion can lead to increased levels of acetol, which can spontaneously form MGO (79).

\section{Minor pathways of methylglyoxal detoxification}

The family of aldehyde dehydrogenase (AD) consists of three isoforms, which are grouped dependent on their intracellular locations (80). Aldehyde dehydrogenase 1 (cytosolic) and aldehyde dehydrogenase 2 (mitochondrial) are the predominant isozymes (81), while aldehyde dehydrogenase 3 (cytosolic) is the least abundant (82). All the aldehyde dehydrogenases are able to catalyze the NAD-dependent oxidation of MGO into pyruvate (83). However most aldehydes which are detoxified by this pathway are unhydrated at physiological $\mathrm{pH}$. Oxoaldehydes such as MGO however are mainly present in the hydrated form, which may explain why MGO is only a poor substrate of AD (80). The enzyme 2oxoaldehyde dehydrogenase was first purified from sheep liver, and found to catalyze the oxidation of MGO to pyruvate in the presence of an amino-alcohol as enzyme activator (84). During this reaction, which is specific for the metabolism of $\alpha$-oxoaldehydes, NAD or NADPH is needed as a cofactor. However, due to its low abundance and its unknown activator in vivo there is only limited literature about this enzyme reaction and its contribution to the detoxification of MGO therefore stays largely unknown (85).

\section{Modification of proteins and nucleic acids by methylglyoxal}

MGO can react with lysine and arginine residues in proteins. It has been reported that MGO primarily reacts with arginine residues to form imidazolones: $\mathrm{N}_{\delta}$ - 5 -hydro-5-methyl4-imidazolon-2-yl)-L-ornithine (MG-H1), 2-amino-5-(2-amino-5-hydro-5-methyl-4imidazolon-1-yl)-pentanoic acid (MG-H2) and 2-amino-5-(2-amino-4-hydro-4-methyl-5imidazolon-1-yl)-pentanoic acid (MG-H3) (86-88). With arginine, MGO also forms $\mathrm{N}_{\delta}-(5-$ 
hydroxy-4,6-dimethylpyramidine-2-yl)-L-ornithine (arginine-pyrimidine, AP) (89), and $\mathrm{N}_{\delta^{-}}$ (4-carboxy-4,6-dimethyl-5,6-dihydroxy-1,4,5,6-tetrahydropyrimidine-2-yl)-L-ornithine

(tetrahydropyrimidine, THP) (90). MGO reacts with lysine residues to $\mathrm{N}_{\varepsilon}-(1-$ carboxyethyl)lysine (CEL) (91), and the MGO derived dimer 1,3-di( $\mathrm{N}_{\varepsilon}$-lysino)-4-methylimidazolium (MOLD) (92). In addition it also can react with one lysine and one arginine to the adduct 2-ammonia-6-(\{2-[4-ammonio-5-oxido-5-oxopentyl)amino]-4methyl-4,5dihydro-1H-imidazol-5-ylidene\}ino)hexanoate (MODIC) (93).

Besides amino acids, MGO can also modify nucleic acids. The first evidence of the binding of MGO to DNA was obtained in an in vitro experiment in which DNA was incubated with radioactive labelled MGO (94). With the use of ${ }^{32} \mathrm{P}$-postlabelling technique, the cyclic adducts of MGO and 2'-deoxyguanosine have been determined in human lymphocytes and human epithelial cells (95). In vivo, DNA glycation products have first been described by Pischetsrieder who identified N2-carboxyethyl-2'-deoxyguanosin with an antibody in human urine (96). More recently, more sensitive assays like quantitative liquid chromatography electrospray ionization tandem mass spectrometry have been used to screen DNA glycation products in vivo (97). With the use of this tool, the importance and use of these DNA biomarkers can be further elucidated. Furthermore, in the nucleus, DNA can also be considered as an important carbonyl precursor. Oxidation reactions release sugar degradation products from the DNA backbone, which then, may directly glycate the DNA (98).

\section{Oxidative stress formation by methylglyoxal}

Besides a major precursor in the formation of AGEs, MGO is probably also a key factor in the acceleration of oxidative stress. Additional free radicals can be generated from MGO via various ways. Studies have shown that during the conversion of aminoacetone and acetol to $\mathrm{MGO}, \mathrm{H}_{2} \mathrm{O}_{2}$ can be formed. In addition the breakdown of MGO also produces free radicals. Similarly to monosaccharides, MGO is subjected to auto-oxidation (99-101) and can thereby generate ROS. However, these MGO-dependent pathways of ROS formation have only a minor contribution to the overall status of oxidative stress. The major route by which MGO generates ROS is by the direct modification of proteins, like mitochondrial and antioxidant enzymes, and by activating pathways which have not been completely elucidated yet. When cultured cells are incubated with MGO, ROS formation can be detected by measuring the rise of oxidation sensitive fluorescence of 5-carboxy$2^{\prime}, 7^{\prime}$-dichlorodihydrofluorescin-diacetate (DCFH-DA). This phenomenon has been observed in several cell cultures (102-104). Additional proof that MGO and ROS are two closely related and important regulators of hyperglycaemia-induced damage is shown by a recent study of Schlotterer et al. performed in the nematode C. elegans (105). This study shows that detoxification of MGO by GLO-I overexpression prevents hyperglycaemia- 
induced oxidative stress, and thereby improves lifespan of this nematode model. Overall, these data show that MGO is also an important source of ROS, besides that its formation is dependent on ROS, thereby creating a strong vicious circle.

\section{Mechanisms of action}

Despite the various mechanisms of detoxification of $\mathrm{MGO}$, some diseases still lead to elevated levels of MGO and thus MGO-derived AGEs. AGEs are then able to exert detrimental effects, and there are mainly three different pathways proposed by which they act (see figure 1.4). These pathways, with a focus on the precursor MGO, will now be described.

\section{Extracellular matrix modification}

The formation of AGEs in the extracellular matrix (ECM) occurs predominantly on proteins with a slow turnover rate. The major matrix proteins collagen, elastin, vitronectin and laminin are targets to be glycated via the formation of cross-links (48-50). This crosslinking increases the ECM area, which results in increased stiffness of the vasculature (5153). AGE formation can also alter the binding function of the ECM. A study of Dobler et al. showed increased modification of vascular basement membrane type IV collagen by MGO, causing endothelial cell detachment, anoikis, and inhibition of angiogenesis. The hotspot sites of modification were in RGD and GFOGER integrin-binding sites of collagen (106). In addition, a study in diabetic rats showed that neurotrophin-stimulated neurite outgrowth and preconditioned neurite outgrowth was also impaired due to glycation of ECM proteins laminin and fibronectin, thereby linking ECM modification to neuropathy (107).

\section{Receptor-mediated signalling}

The presence of a receptor for AGEs was first described by Esposito and colleagues in a study in endothelial cells, although they did not elucidate the specific cellular interaction sites (108). Nowadays, several receptors have been identified which are able to bind AGEs, including the AGE receptor complex (OST-48, 80K-H, and galectin-3) (109), macrophage scavenger receptors type I and II (110), CD-36 (111) and LOX-I (112). The best characterised receptor for AGE is the receptor for AGE (RAGE) (113).

RAGE is as a signal transduction receptor, which stimulates secondary messenger pathways such as protein kinase C. After this activation, the key target NF-kB is translocated to the nucleus were it increases transcription of various proteins including intracellular adhesion molecule-1 (ICAM-1), E-selectin, tissue factor, vascular endothelial growth factor (VEGF), proinflammatory cytokines and also RAGE itself (114). Despite the fact that the precise AGE ligands for RAGE are still largely unknown, several studies show that RAGE activation is an important step in the development of cardiovascular diseases. 
Prevention of RAGE activation by infusion with the soluble form of RAGE (SRAGE) or knocking out the RAGE gene prevents atherosclerosis in apolipoprotein E-null mice (115; 116), improves diabetic wound healing (117), and prevents nephropathy (118), and early retinopathy (119).

RAGE is not only selective for AGEs. Also other ligands like S100, high-mobility group protein B1, macrophage-1 antigen, amphoterin and $\beta$-amyloid can bind to RAGE and activate similar signalling pathways (120). Within the heterogeneous group of AGEs, not all AGEs are important ligands for RAGE. Nevertheless, the major AGE NE-(1carboxymethyl)lysine (CML) has been identified as a RAGE binding modification, resulting in an increased expression of vascular adhesion molecule-1 (VCAM-1) and ICAM-1, thereby facilitating the binding and extravasation of monocytes (62; 121-123).Interaction of MGO-modified albumin with monocytes or macrophages results in activation and subsequent synthesis and secretion of tumour necrose factor alpha (TNF- $\alpha$ ), interleukin$1 \beta$, and monocyte colony stimulating factor (124-126). This can indirectly result in endothelial activation and chemotaxis of leucocytes. Also a study by Lieuw-a-Fa et al. shows an interaction of MGO-modified albumin with macrophages, however binding to endothelial cells could not been observed (127). Therefore the existence of a direct effect of MGO-derived AGEs on endothelial cells is still debated.

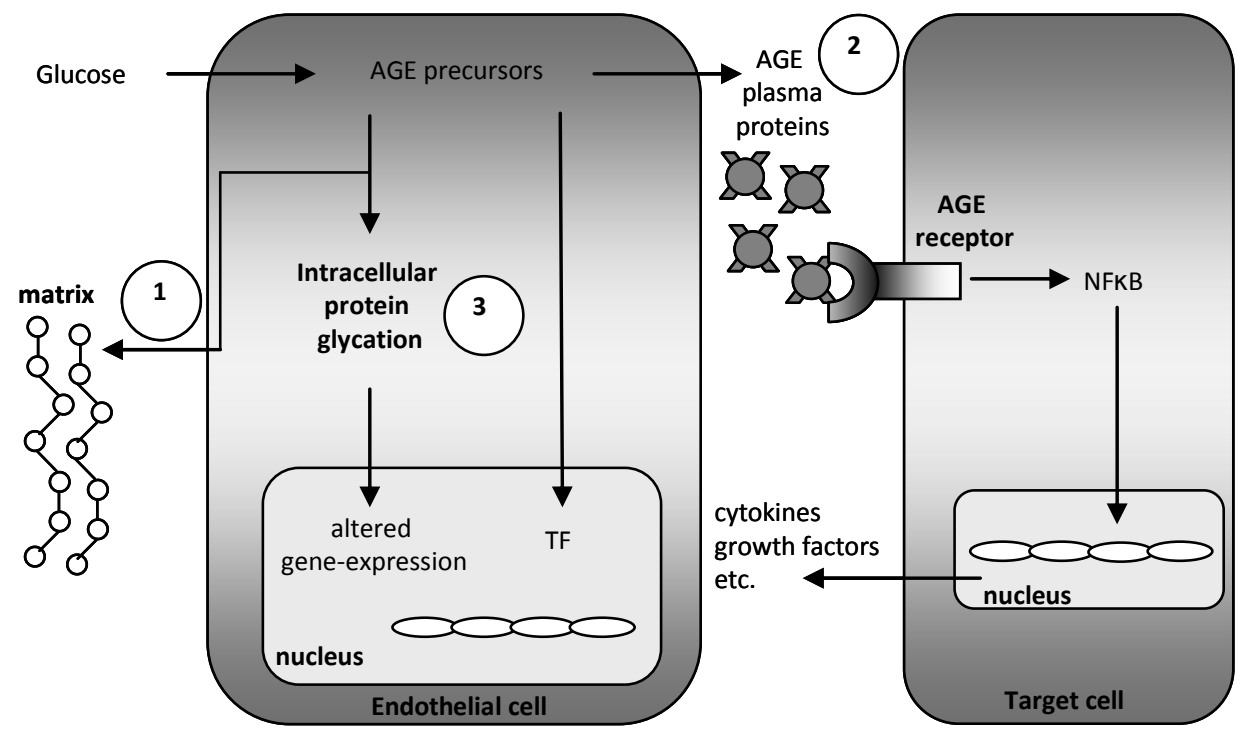

Figure 1.4. Mechanisms thought to mediate advanced glycation endproduct-induced damage

One pathway via which advanced glycation endproduct can lead to damage is by glycation of extracellular matrix components [1]. Furthermore AGEs in the plasma can also activate the receptor for AGE and thereby induce changes in gene expression of target cells like, among others, smooth muscle, mesangial and retinal cells [2]. In addition AGE precursors can damage vascular cells by modification of intracellular proteins, thereby altering their function and even initiate altered gene expression [3]. (figure modified from Brownlee (10)). 


\section{Intracellular modification}

As a result of their generation from dicarbonyls derived from glycolysis, AGEs can often accumulate intracellularly (128). It is plausible that besides modifying the function of intracellular proteins, these AGEs can also induce a cascade of intracellular signalling pathways. From the three dicarbonyl compounds MGO, GO and 3-DG, MGO is believed to be the most important intracellular precursor of glycation (65).

The effect of intracellular sugars on protein function was first shown by a study of Giardino and co-workers. They showed that basic fibroblast growthfactor is one of the main AGE-modified proteins in endothelial cells, which markedly decreases its mitogenic activity (128). Since then several proteins have been shown to be glycated, with MGO as the major precursor in this process. In both tumour cells and endothelial cells heat-shock protein 27 has been identified as a major MGO-modified protein (129; 130). Other important proteins modified by MGO are mitochondrial membrane proteins (63) and antioxidant enzymes (64), resulting in increased oxidative stress.

An interesting new concept about how MGO can influence cell metabolism is by the modification of transcription factors. Yao et al. demonstrated that in mouse kidney endothelial cells, high glucose causes increased MGO modification of the co-repressor $\mathrm{mSin} 3 \mathrm{~A}$. This modification results in increased angiopoietin-2 expression and subsequently expression of ICAM-1 and VCAM-1 (131). Similarly hyperglycaemia-induced elevated MGO levels modify co-activator p300, which causes impaired hypoxia-induced factor (HIF)-1 alpha binding resulting in decreased VEGF expression in cases of hypoxia (132). In addition to the altered gene expression pathways, intracellular AGE accumulation can also directly influence other signalling pathways. Bucala et al. showed that the bioavailability of NO in the vascular bed can be significantly decreased by a direct chemical reaction with AGE modified proteins, thereby blocking its antiproliferative and vasorelaxing effects (133; 134). In addition, a recent paper by Queisser et al. demonstrates that hyperglycaemiainduced formation of MGO covalently modifies the $20 \mathrm{~S}$ proteasome, thereby decreasing its activity in the diabetic kidney (135).

\section{Scavengers of methylglyoxal}

Besides the neutralization of MGO by endogenous detoxification enzymes pharmacological tools are also available to prevent AGE accumulation and MGO-induced damage. Three of the most used and well known scavengers of MGO will therefore be discussed.

\section{Aminoguanidine}

Aminoguanidine ( $A G)$ is a $\alpha, \beta$-dicarbonyl scavenging agent with two key reaction centres: the nucleophilic hydrazine group $\left(-\mathrm{NHNH}_{2}\right)$ and the dicarbonyl directing guanidino group (- 
$\mathrm{NH}-\mathrm{C}$ ) $=\mathrm{NH}) \mathrm{NH}_{2}$ ). The AGE-lowering characteristics of these groups were first described by Brownlee et al. (136). In experimental diabetes, AG has been proven to be very effective in lowering AGEs and preventing diabetic complications like nephropathy (36; 137), neuropathy (138), and retinopathy (35).

However it should be noted that $A G$ is not a specific MGO scavenger since it also reacts with other carbonyl metabolites, such as 3-DG, MDA, HNE and formaldehyde (136; 139). In addition, AG is a well known nitric oxide synthase (NOS) inhibitor. NOS catalyze the production of NO from L-arginine (140). Thus, AG would inhibit NO formation when used to scavenge MGO. Furthermore AG also inhibits semicarbazide-sensitive amine oxidase activity, thereby preventing MGO production, although formation from aminoacetone is thought to be just a minor source of MGO (141). Besides this, AG also acts as an antioxidant, which is possible independently of the prevention of AGE formation (142). Therefore, results obtained from experiments performed with AG should be interpreted with care and cannot be attributed exclusively to inhibition of AGEs.

The use of $A G$ in clinical trials resulted in disappointing effects. Clinical trials of $A G$ were performed in patients with overt diabetic nephropathy (ACTION); ACTION I was conducted in patients with T1DM and ACTION II in patients with T2DM. The primary endpoint was, in both studies, the time from randomization (in placebo, low- and high-dose AG) to the doubling of baseline serum creatinine. In ACTION I, the combined AG dose group showed a tendency towards slower doubling of serum creatinine, but this was not statistically significant (143). The ACTION II study was terminated earlier due to safety concerns and lack of efficacy. The reported side effects in this study were gastrointestinal disturbance, abnormal liver function, flu-like symptoms and a rare vasculitis (144).

\section{Alagebrium}

Alagebrium (formerly known as ALT-711) is an AGEs cross-link breaker. It reduces collagen cross-linking in diabetic animals by cleaving the bonds between adjacent carbonyl groups of cross-linked proteins (145). Alagebrium is the only AGE cross-link breaker which is being tested in human clinical trials (146). Moreover, evidence suggests that alagebrium directly scavenges MGO. In a study conducted by Nobecourt et al. alagebrium did not reverse MGO-mediated cross-linking of apolipoprotein (Apo) A-I, the main apolipoprotein of high density lipoprotein. However, alagebrium prevented MGO-mediated modification of ApoA-I (147). This indicates that alagebrium is a MGO scavenger, although the mechanism is still unknown.

\section{Metformin}

The biguanide metformin was already introduced into clinical practice in 1957 as an oral antihyperglycaemic agent for the management of T2DM. The blood glucose-lowering 
actions of metformin result primarily from reducing appetite, decreasing gastrointestinal absorption of carbohydrates, inhibiting hepatic gluconeogenesis, and increasing cellular uptake of glucose (148). The potential additional benefit expected from its antihyperglycaemic effect was recognized more recently (149). One of these additional beneficial effects is that metformin was observed to trap reactive carbonyls like MGO and GO (150). Moreover, administration of metformin in patients with T2DM not only reduced MGO production but also enhanced MGO degradation by increasing the activity of the MGO-detoxifying enzyme glyoxalase (151).

\section{The involvement of advanced glycation endproducts in endothelial dysfunction and consequently vascular complications}

\section{Endothelial dysfunction}

Endothelial cells form a continuous layer on the intimal surface of the entire cardiovascular system. Under physiological circumstances endothelial cells efficiently regulate basal vascular tone and vascular reactivity by responding to mechanical forces and neuro-humoral mediators by the release of a variety of relaxing and contracting factors (152). The endothelium-derived relaxing factors include NO, prostacyclins and one or several, as yet elusive, endothelium-derived hyperpolarizing factors (EDHF) (153). Besides the vascular tone the endothelium also regulates permeability, the composition of the sub-endothelial matrix, the adhesion and extravasations of leucocytes and the balance between coagulation and fibrinolysis (154). This regulation is accomplished by a strong interplay between the endothelial cells and other cell types, like smooth muscle cells and leukocytes. The term endothelial dysfunction describes the situation in which the endothelium is no longer capable in regulating these functions in either the basal state or after stimulation.

There are several tools to measure endothelial function in vitro and in vivo. In vitro cultured endothelial cells can be very useful for in-depth investigation of cell signalling under pathological conditions. However it is unclear how closely they truly resemble human arterial endothelial cells in vivo. Therefore the ex vivo use of whole arterial preparations in a organ bath is probably more accurate. The major advantage of this system is that actually the function of the endothelium can be measured by monitoring relaxation of the artery in response to agonists. In vivo vascular relaxation can be measured by several techniques, although no gold standard exists (155). The most popular technique is brachial artery imaging coupled with reactive hyperaemia. Hyperaemia results in increased blood flow, shear stress and NO-mediated vasodilatation, which is known as flow-mediated dilatation (156). Furthermore, endothelial activation, i.e. the 
acquisition of inflammatory properties by the endothelium, can be evaluated by measuring circulating levels of soluble ICAM-1, VCAM-1, and E-selectin; von Willebrand factor; tissue plasminogen activator; PAI-1; and circulating mature endothelial cells and endothelial progenitor cells (157).

Indeed, measurement of endothelial function points out that endothelium-dependent vasorelaxation is most often impaired in diabetic patients $(158 ; 159)$ and also in animals models of diabetes $(160 ; 161)$. In addition circulating markers of endothelial dysfunction are increased in people with diabetes (162). Furthermore also characteristics of the vascular wall, like increased stiffness (163) and intima-media thickness (164) correlate with diabetes. Loss of proper endothelial function is believed to be a critical initiating factor in the development of vascular complications. Indeed a large body of evidence indicates that endothelial dysfunction is closely associated with the development of diabetic retinopathy, nephropathy and atherosclerosis in both T1DM and T2DM (165-167). Moreover, some studies show that signs of endothelial dysfunction can already be present before the onset of diabetic nephropathy (168-170).

\section{Vascular complications}

Several studies showed that glycation is strongly involved in hyperglycaemia-induced endothelial dysfunction and consequently the development of vascular complications. Therefore the following section of this introduction will describe the role of AGEs and endothelial dysfunction in macro- and microvascular complications, including atherosclerosis, nephropathy, retinopathy and neuropathy.

\section{Cardiovascular disease}

AGEs are elevated in the serum of T2DM with coronary heart disease (171). Furthermore they have been localised in atherosclerotic lesions, fatty streaks, lipid-containing smooth muscle cells and macrophages in people with diabetes, and the levels of tissue AGEs correlate with the severity of the lesions (172).

The formation of AGEs can be linked to atherosclerosis in multiple ways, such as via the increase of low density lipoprotein (LDL) levels, destabilisation of plaques via effects on matrix metalloproteinases, and inhibition of vascular repair. Furthermore, also the development of endothelial dysfunction plays an important role due to stiffening and narrowing of the vasculature. Indeed, soluble plasma proteins, such as LDL and immunoglobulins (Ig)G, are entrapped and covalently cross-linked by AGEs on collagen $(173 ; 174)$. The glycation process occurs both on the apoprotein $B(a p o B)$ and on phospholipid components of LDL, leading to both functional alterations in LDL clearance and increased susceptibility to oxidative modifications (175). Further, the uptake of glycated LDL by human monocyte-derived macrophages occurs to a greater extent than 
for native LDL by a low-affinity nonspecific ("scavenger") receptor with the resulting stimulation of "foam" cell formation, characteristic of the early atherosclerotic lesion (176). In addition, transforming growth factor- $\beta$ (TGF- $\beta$ ) might act as an intermediate factor in AGE-induced fibronectin production by smooth muscle cells (177).

Several animal studies show the involvement of RAGE in the pathogenesis of atherosclerosis. Research in diabetic mice with a deficiency in apoE, which thereby develop spontaneous atherosclerosis, showed that the administration of sRAGE suppressed accelerated diabetic atherosclerosis. Interestingly, euglycaemic animals receiving SRAGE also demonstrated a trend towards diminished atherosclerosis compared to vehicle-treated animals $(115 ; 178)$. In addition, also diabetic double knock out mice for RAGE and apoE show less plaque accumulation than diabetic apoE(-/-) mice (116). The role of the AGE precursor MGO in atherosclerosis is still largely unknown.

\section{Diabetic nephropathy}

The kidney is a major target for the development of hyperglycaemia-induced damage. In diabetic nephropathy, injury to both the glomerulus and the tubulointerstitium is accompanied by increased protein excretion and consequently a decline in renal function (179). It is likely that diabetic nephropathy occurs as a result of an interplay of haemodynamic and metabolic factors (180). Because of the important role of the kidneys in the clearance of AGEs, there is a high amount of circulating AGEs at this site. Besides the significant increase in urinary excretion of AGEs, diabetic animals models have also shown that renal AGEs are elevated. Indeed the characteristic structural changes of diabetic nephropathy, thickened glomerular basement membrane and mesangial expansion, are accompanied by accumulation of AGEs, leading to glomerulosclerosis and interstitial fibrosis (181). Furthermore administration of AGE-albumin resulted in characteristics of diabetic nephropathy (182), and a study in diabetic mice shows that sRAGE injection prevents diabetic nephropathy (183).

In streptozotocin-induced diabetes in rats, treatment with the AGE-lowering agent aminoguanidine is known to prevent nephropathy, indicating a role of glycation in the development of this pathogenesis (137). In addition, Beisswenger et al. showed that progression of diabetic nephropathy is significantly related to elevation in dicarbonyl stress (184).

\section{Diabetic retinopathy}

The prevalence of diabetic retinopathy in patients with T1DM with a duration of 10 years is around $80 \%$, and this increases to approximately $95 \%$ by 20 years of diabetes $(185 ; 186)$. Although diabetic retinopathy is slightly less common in T2DM patients, it is still a frequent microvascular complication in this group (185). The failure to regulate blood 
glucose levels leads to a range of pathogenic lesions in the retina and other vascular beds which consequently results in macular edema, ischaemia and neovascularisation (187). Research in animals models showed that good glycaemic control, after an initial phase of poor control, is not protective against the development of retinopathy (188; 189). One explanation for this irreversible early effect is the formation of AGEs. Indeed several studies showed an increase of AGEs in retinal vessels and neuroglia of diabetic patients (190-192). Furthermore infusion of AGEs shows an upregulation of retinal VEGF, which is important in the development of neovascularisation and retinal barrier dysfunction (193; 194). Retinal vascular endothelial cells exposed to AGEs show abnormal eNOS expression, which may account for some of the vasoregulatory abnormalities seen in the retinal microcirculation in diabetes (195).

The inhibition of AGE formation with aminoguanidine showed that retinopathy can be prevented, which provides evidence for a role of AGEs in the development of diabetic retinopathy $(35 ; 196 ; 197)$.

\section{Diabetic neuropathy}

Several studies have shown that glycation may play a role in the development of neuropathy. A study by Brownlee et al. demonstrated that AGEs on myelin can quench immunoglobulins and elicit immunological responses that may lead to demyelisation (198). This was supported by recent studies that demonstrated pronounced AGE immunoreactivity on axons and myelin sheaths in $90 \%$ of type 2 diabetic patients with neuropathy (199). In addition, AGE accumulation on laminin and collagen type IV was shown to be involved in the impaired regeneration of dorsal root ganglia (200). Furthermore accumulation of skin AGEs correlates with both clinical and pre-clinical signs of autonomic and sensory diabetic neuropathy (201). A study by Duran-Jimenez et al. shows that ECM modification by MGO is the main glycation pathway involved in a reduction in neurotrophin-stimulated neurite outgrowth and preconditioned neurite outgrowth (107). In agreement, scavenging of oxo-aldehydes with aminoguanidine has been shown to prevent the decrease in, both motor and sensory nerve conduction velocity that is associated with experimental diabetes (202).

There is also evidence that RAGE is involved in nerve dysfunction. Bierhaus et al. have demonstrated that ligands of RAGE activated NF-KB, and increased the expression of interleukin- 6 in the microvasculature of sural nerves from individuals with diabetic neuropathy (203). Another study has shown that dorsal root ganglia neurons express functional RAGE and respond to ligands for RAGE with downstream signalling, increased oxidative stress and cellular injury (204). 


\section{Thesis objectives and outline}

Since their discovery in early 1900, AGEs have been the focus of much research. Although there is no doubt about a role of AGEs in the development of diabetic vascular complications, the precise modifications and pathways involved still remain to be elucidated. Therefore the central aim of this thesis was to investigate the role of intracellular MGO and MGO-derived AGEs in the development of endothelial dysfunction and consequently vascular complications. In this thesis Chapter $\mathbf{2}$ and Chapter $\mathbf{3}$ describe the work that has been done to understand how MGO or MGO derived-AGEs can interfere with vascular function in vitro.

The development of diabetic vascular complications in vivo can be investigated in a commonly used animal model of T1DM, the streptozotocin-induced diabetic rat. Researchers who have focused on the role of the main precursor MGO and MGO-derived AGEs in the development of diabetic vascular complications have mainly conducted research by making use of the rather non-selective $\alpha$-oxo-aldehyde scavenger aminoguanidine in animal models. However, with the availability of a novel in vivo GLO-I overexpression model in rats it becomes possible to specifically address the involvement of MGO in the development of vascular complications. Chapter 4 describes the phenotype of this in vivo animal model with and without diabetes. The effect of MGO lowering in these diabetic animals on hyperglycaemia-induced vascular and renal dysfunction is described in Chapter 5. Chapter 6 reports on improved haemodynamics by GLO-I overexpression. The general discussion, conclusions and possibilities for further research are given in Chapter 7. 


\section{References}

1. Clark CM, Jr., Lee DA: Prevention and treatment of the complications of diabetes mellitus. N Engl J Med 332:1210-1217, 1995

2. Wild S, Roglic G, Green A, Sicree R, King H: Global prevalence of diabetes: estimates for the year 2000 and projections for 2030. Diabetes Care 27:1047-1053, 2004

3. The effect of intensive treatment of diabetes on the development and progression of long-term complications in insulin-dependent diabetes mellitus. The Diabetes Control and Complications Trial Research Group. N Engl J Med 329:977-986, 1993

4. Intensive blood-glucose control with sulphonylureas or insulin compared with conventional treatment and risk of complications in patients with type 2 diabetes (UKPDS 33). UK Prospective Diabetes Study (UKPDS) Group. Lancet 352:837-853, 1998

5. Mitchell GF, Hwang SJ, Vasan RS, Larson MG, Pencina MJ, Hamburg NM, Vita JA, Levy D, Benjamin EJ: Arterial stiffness and cardiovascular events: the Framingham Heart Study. Circulation 121:505-511

6. Cameron NE, Eaton SE, Cotter MA, Tesfaye S: Vascular factors and metabolic interactions in the pathogenesis of diabetic neuropathy. Diabetologia 44:1973-1988, 2001

7. Fong DS, Sharza M, Chen W, Paschal JF, Ariyasu RG, Lee PP: Vision loss among diabetics in a group model Health Maintenance Organization (HMO). Am J Ophthalmol 133:236-241, 2002

8. Schrijvers BF, De Vriese AS, Flyvbjerg A: From hyperglycaemia to diabetic kidney disease: the role of metabolic, haemodynamic, intracellular factors and growth factors/cytokines. Endocr Rev 25:971-1010, 2004

9. Malik VS, Popkin BM, Bray GA, Despres JP, Hu FB: Sugar-sweetened beverages, obesity, type 2 diabetes mellitus, and cardiovascular disease risk. Circulation 121:1356-1364

10. Brownlee M: Biochemistry and molecular cell biology of diabetic complications. Nature 414:813-820, 2001

11. Bravi MC, Pietrangeli P, Laurenti O, Basili S, Cassone-Faldetta M, Ferri C, De Mattia G: Polyol pathway activation and glutathione redox status in non-insulin-dependent diabetic patients. Metabolism 46:1194-1198, 1997

12. Williamson JR, Chang K, Frangos M, Hasan KS, Ido Y, Kawamura T, Nyengaard JR, van den Enden M, Kilo C, Tilton RG: Hyperglycemic pseudohypoxia and diabetic complications. Diabetes 42:801-813, 1993

13. Altan VM: The pharmacology of diabetic complications. Curr Med Chem 10:1317-1327, 2003

14. Drel VR, Pacher P, Ali TK, Shin J, Julius U, El-Remessy AB, Obrosova IG: Aldose reductase inhibitor fidarestat counteracts diabetes-associated cataract formation, retinal oxidative-nitrosative stress, glial activation, and apoptosis. Int J Mol Med 21:667-676, 2008

15. Oates PJ, Mylari BL: Aldose reductase inhibitors: therapeutic implications for diabetic complications. Expert Opin Investig Drugs 8:2095-2119, 1999

16. Iso K, Tada H, Kuboki K, Inokuchi T: Long-term effect of epalrestat, an aldose reductase inhibitor, on the development of incipient diabetic nephropathy in Type 2 diabetic patients. J Diabetes Complications 15:241-244, 2001

17. Johnson BF, Nesto RW, Pfeifer MA, Slater WR, Vinik Al, Chyun DA, Law G, Wackers FJ, Young LH: Cardiac abnormalities in diabetic patients with neuropathy: effects of aldose reductase inhibitor administration. Diabetes Care 27:448-454, 2004

18. Passariello N, Sepe J, Marrazzo G, De Cicco A, Peluso A, Pisano MC, Sgambato S, Tesauro P, D'Onofrio F: Effect of aldose reductase inhibitor (tolrestat) on urinary albumin excretion rate and glomerular filtration rate in IDDM subjects with nephropathy. Diabetes Care 16:789-795, 1993

19. Lee TS, MacGregor LC, Fluharty SJ, King GL: Differential regulation of protein kinase C and (Na,K)-adenosine triphosphatase activities by elevated glucose levels in retinal capillary endothelial cells. J Clin Invest 83:90-94, 1989

20. Ishii H, Jirousek MR, Koya D, Takagi C, Xia P, Clermont A, Bursell SE, Kern TS, Ballas LM, Heath WF, Stramm LE, Feener EP, King GL: Amelioration of vascular dysfunctions in diabetic rats by an oral PKC beta inhibitor. Science 272:728-731, 1996

21. Koya D, King GL: Protein kinase C activation and the development of diabetic complications. Diabetes 47:859866,1998

22. Lee TS, Saltsman KA, Ohashi H, King GL: Activation of protein kinase $C$ by elevation of glucose concentration: proposal for a mechanism in the development of diabetic vascular complications. Proc Natl Acad Sci U S A 86:5141-5145, 1989 
23. Sheetz MJ, King GL: Molecular understanding of hyperglycaemia's adverse effects for diabetic complications. JAMA 288:2579-2588, 2002

24. Koya D, Haneda M, Nakagawa $H$, Isshiki K, Sato $H$, Maeda S, Sugimoto T, Yasuda H, Kashiwagi A, Ways DK, King GL, Kikkawa R: Amelioration of accelerated diabetic mesangial expansion by treatment with a PKC beta inhibitor in diabetic $\mathrm{db} / \mathrm{db}$ mice, a rodent model for type 2 diabetes. FASEB J 14:439-447, 2000

25. Zachara NE, Hart GW: Cell signaling, the essential role of O-GlcNAc! Biochim Biophys Acta 1761:599-617, 2006

26. Kreppel LK, Blomberg MA, Hart GW: Dynamic glycosylation of nuclear and cytosolic proteins. Cloning and characterization of a unique O-GIcNAc transferase with multiple tetratricopeptide repeats. J Biol Chem 272:93089315, 1997

27. Lubas WA, Frank DW, Krause M, Hanover JA: O-Linked GlcNAc transferase is a conserved nucleocytoplasmic protein containing tetratricopeptide repeats. J Biol Chem 272:9316-9324, 1997

28. Kornfeld S, Kornfeld R, Neufeld EF, O'Brien PJ: The Feedback Control of Sugar Nucleotide Biosynthesis in Liver. Proc Natl Acad Sci U S A 52:371-379, 1964

29. Federici M, Menghini R, Mauriello A, Hribal ML, Ferrelli F, Lauro D, Sbraccia P, Spagnoli LG, Sesti G, Lauro R: Insulin-dependent activation of endothelial nitric oxide synthase is impaired by O-linked glycosylation modification of signaling proteins in human coronary endothelial cells. Circulation 106:466-472, 2002

30. Du XL, Edelstein D, Dimmeler S, Ju Q, Sui C, Brownlee M: Hyperglycaemia inhibits endothelial nitric oxide synthase activity by posttranslational modification at the Akt site. J Clin Invest 108:1341-1348, 2001

31. Walgren JL, Vincent TS, Schey KL, Buse MG: High glucose and insulin promote O-GlcNAc modification of proteins, including alpha-tubulin. Am J Physiol Endocrinol Metab 284:E424-434, 2003

32. Du XL, Edelstein D, Rossetti L, Fantus IG, Goldberg H, Ziyadeh F, Wu J, Brownlee M: Hyperglycaemia-induced mitochondrial superoxide overproduction activates the hexosamine pathway and induces plasminogen activator inhibitor-1 expression by increasing Sp1 glycosylation. Proc Natl Acad Sci U S A 97:12222-12226, 2000

33. Maillard LC: Action des acides aminés sur les sucres: formation des melanoidines par voie méthodique. C.R. Hebd. Seances Acad. Sci. 154:66-68., 1912

34. Rahbar S, Blumenfeld O, Ranney HM: Studies of an unusual hemoglobin in patients with diabetes mellitus. Biochem Biophys Res Commun 36:838-843, 1969

35. Hammes HP, Martin S, Federlin K, Geisen K, Brownlee M: Aminoguanidine treatment inhibits the development of experimental diabetic retinopathy. Proc Natl Acad Sci U S A 88:11555-11558, 1991

36. Soulis-Liparota T, Cooper M, Papazoglou D, Clarke B, Jerums G: Retardation by aminoguanidine of development of albuminuria, mesangial expansion, and tissue fluorescence in streptozocin-induced diabetic rat. Diabetes 40:1328-1334, 1991

37. Nakamura S, Makita Z, Ishikawa S, Yasumura K, Fujii W, Yanagisawa K, Kawata T, Koike T: Progression of nephropathy in spontaneous diabetic rats is prevented by OPB-9195, a novel inhibitor of advanced glycation. Diabetes 46:895-899, 1997

38. Singh R, Barden A, Mori T, Beilin L: Advanced glycation end-products: a review. Diabetologia 44:129-146, 2001

39. Verzijl N, DeGroot J, Oldehinkel E, Bank RA, Thorpe SR, Baynes JW, Bayliss MT, Bijlsma JW, Lafeber FP, Tekoppele JM: Age-related accumulation of Maillard reaction products in human articular cartilage collagen. Biochem J 350 Pt 2:381-387, 2000

40. Thornalley PJ, Langborg A, Minhas HS: Formation of glyoxal, methylglyoxal and 3-deoxyglucosone in the glycation of proteins by glucose. Biochem J 344 Pt 1:109-116, 1999

41. Esterbauer H, Gebicki J, Puhl H, Jurgens G: The role of lipid peroxidation and antioxidants in oxidative modification of LDL. Free Radic Biol Med 13:341-390, 1992

42. Esterbauer H, Schaur RJ, Zollner H: Chemistry and biochemistry of 4-hydroxynonenal, malonaldehyde and related aldehydes. Free Radic Biol Med 11:81-128, 1991

43. Ceriello A: New insights on oxidative stress and diabetic complications may lead to a "causal" antioxidant therapy. Diabetes Care 26:1589-1596, 2003

44. Nishikawa T, Edelstein D, Du XL, Yamagishi S, Matsumura T, Kaneda Y, Yorek MA, Beebe D, Oates PJ, Hammes HP, Giardino I, Brownlee M: Normalizing mitochondrial superoxide production blocks three pathways of hyperglycaemic damage. Nature 404:787-790, 2000

45. Du X, Matsumura T, Edelstein D, Rossetti L, Zsengeller Z, Szabo C, Brownlee M: Inhibition of GAPDH activity by poly(ADP-ribose) polymerase activates three major pathways of hyperglycemic damage in endothelial cells. J Clin Invest 112:1049-1057, 2003 
46. Giugliano D, Ceriello A, Paolisso G: Oxidative stress and diabetic vascular complications. Diabetes Care 19:257-267, 1996

47. Miwa S, St-Pierre J, Partridge L, Brand MD: Superoxide and hydrogen peroxide production by Drosophila mitochondria. Free Radic Biol Med 35:938-948, 2003

48. Mokini Z, Marcovecchio ML, Chiarelli F: Molecular pathology of oxidative stress in diabetic angiopathy: role of mitochondrial and cellular pathways. Diabetes Res Clin Pract 87:313-321

49. Laursen JB, Somers M, Kurz S, McCann L, Warnholtz A, Freeman BA, Tarpey M, Fukai T, Harrison DG: Endothelial regulation of vasomotion in apoE-deficient mice: implications for interactions between peroxynitrite and tetrahydrobiopterin. Circulation 103:1282-1288, 2001

50. Fridovich I: Superoxide radical and superoxide dismutases. Annu Rev Biochem 64:97-112, 1995

51. Muzykantov VR: Targeting of superoxide dismutase and catalase to vascular endothelium. J Control Release 71:1-21, 2001

52. Seifried HE, Anderson DE, Fisher EI, Milner JA: A review of the interaction among dietary antioxidants and reactive oxygen species. J Nutr Biochem 18:567-579, 2007

53. Cai H, Harrison DG: Endothelial dysfunction in cardiovascular diseases: the role of oxidant stress. Circ Res 87:840-844, 2000

54. Cho KS, Lee EH, Choi JS, Joo CK: Reactive oxygen species-induced apoptosis and necrosis in bovine corneal endothelial cells. Invest Ophthalmol Vis Sci 40:911-919, 1999

55. Szabo C: Role of nitrosative stress in the pathogenesis of diabetic vascular dysfunction. $\mathrm{Br} \mathrm{J} \mathrm{Pharmacol}$ 156:713-727, 2009

56. Marui N, Offermann MK, Swerlick R, Kunsch C, Rosen CA, Ahmad M, Alexander RW, Medford RM: Vascular cell adhesion molecule-1 (VCAM-1) gene transcription and expression are regulated through an antioxidantsensitive mechanism in human vascular endothelial cells. J Clin Invest 92:1866-1874, 1993

57. Uemura S, Matsushita H, Li W, Glassford AJ, Asagami T, Lee KH, Harrison DG, Tsao PS: Diabetes mellitus enhances vascular matrix metalloproteinase activity: role of oxidative stress. Circ Res 88:1291-1298, 2001

58. Cheng JJ, Chao YJ, Wung BS, Wang DL: Cyclic strain-induced plasminogen activator inhibitor-1 (PAI-1) release from endothelial cells involves reactive oxygen species. Biochem Biophys Res Commun 225:100-105, 1996

59. Ciuchi E, Odetti P, Prando R: Relationship between glutathione and sorbitol concentrations in erythrocytes from diabetic patients. Metabolism 45:611-613, 1996

60. Inoguchi T, Li P, Umeda F, Yu HY, Kakimoto M, Imamura M, Aoki T, Etoh T, Hashimoto T, Naruse M, Sano H, Utsumi $\mathrm{H}$, Nawata $\mathrm{H}$ : High glucose level and free fatty acid stimulate reactive oxygen species production through protein kinase C--dependent activation of NAD(P)H oxidase in cultured vascular cells. Diabetes 49:1939-1945, 2000

61. Kaneto H, Xu G, Song KH, Suzuma K, Bonner-Weir S, Sharma A, Weir GC: Activation of the hexosamine pathway leads to deterioration of pancreatic beta-cell function through the induction of oxidative stress. J Biol Chem 276:31099-31104, 2001

62. Basta G, Lazzerini G, Massaro M, Simoncini T, Tanganelli P, Fu C, Kislinger T, Stern DM, Schmidt AM, De Caterina R: Advanced glycation end products activate endothelium through signal-transduction receptor RAGE: a mechanism for amplification of inflammatory responses. Circulation 105:816-822, 2002

63. Rosca MG, Monnier VM, Szweda LI, Weiss MF: Alterations in renal mitochondrial respiration in response to the reactive oxoaldehyde methylglyoxal. Am J Physiol Renal Physiol 283:F52-59, 2002

64. Wu L, Juurlink BH: Increased methylglyoxal and oxidative stress in hypertensive rat vascular smooth muscle cells. Hypertension 39:809-814, 2002

65. Shinohara M, Thornalley PJ, Giardino I, Beisswenger P, Thorpe SR, Onorato J, Brownlee M: Overexpression of glyoxalase-I in bovine endothelial cells inhibits intracellular advanced glycation endproduct formation and prevents hyperglycaemia-induced increases in macromolecular endocytosis. J Clin Invest 101:1142-1147, 1998

66. Thornalley PJ: The glyoxalase system in health and disease. Mol Aspects Med 14:287-371, 1993

67. Rae C, Berners-Price SJ, Bulliman BT, Kuchel PW: Kinetic analysis of the human erythrocyte glyoxalase system using $1 \mathrm{H}$ NMR and a computer model. Eur J Biochem 193:83-90, 1990

68. Phillips SA, Thornalley PJ: The formation of methylglyoxal from triose phosphates. Investigation using a specific assay for methylglyoxal. Eur J Biochem 212:101-105, 1993

69. Richard JP: Kinetic parameters for the elimination reaction catalyzed by triosephosphate isomerase and an estimation of the reaction's physiological significance. Biochemistry 30:4581-4585, 1991

70. Ray S, Ray M: Formation of methylglyoxal from aminoacetone by amine oxidase from goat plasma. J Biol Chem 258:3461-3462, 1983 
71. Pompliano DL, Peyman A, Knowles JR: Stabilization of a reaction intermediate as a catalytic device: definition of the functional role of the flexible loop in triosephosphate isomerase. Biochemistry 29:3186-3194, 1990

72. Reichard GA, Jr., Skutches CL, Hoeldtke RD, Owen OE: Acetone metabolism in humans during diabetic ketoacidosis. Diabetes 35:668-674, 1986

73. Ray M, Ray S: Aminoacetone oxidase from goat liver. Formation of methylglyoxal from aminoacetone. J Biol Chem 262:5974-5977, 1987

74. Baynes JW, Thorpe SR: Glycoxidation and lipoxidation in atherogenesis. Free Radic Biol Med 28:1708-1716, 2000

75. Thornalley PJ: Modification of the glyoxalase system in human red blood cells by glucose in vitro. Biochem $\mathrm{J}$ 254:751-755, 1988

76. Thornalley PJ: Glyoxalase l--structure, function and a critical role in the enzymatic defence against glycation. Biochem Soc Trans 31:1343-1348, 2003

77. Thornalley PJ: Glutathione-dependent detoxification of alpha-oxoaldehydes by the glyoxalase system: involvement in disease mechanisms and antiproliferative activity of glyoxalase I inhibitors. Chem Biol Interact 111-112:137-151, 1998

78. Vander Jagt DL, Robinson B, Taylor KK, Hunsaker LA: Reduction of trioses by NADPH-dependent aldo-keto reductases. Aldose reductase, methylglyoxal, and diabetic complications. J Biol Chem 267:4364-4369, 1992

79. Vander Jagt DL, Hassebrook RK, Hunsaker LA, Brown WM, Royer RE: Metabolism of the 2-oxoaldehyde methylglyoxal by aldose reductase and by glyoxalase-I: roles for glutathione in both enzymes and implications for diabetic complications. Chem Biol Interact 130-132:549-562, 2001

80. Izaguirre G, Kikonyogo A, Pietruszko R: Methylglyoxal as substrate and inhibitor of human aldehyde dehydrogenase: comparison of kinetic properties among the three isozymes. Comp Biochem Physiol B Biochem Mol Biol 119:747-754, 1998

81. Yoshida A, Ikawa M, Hsu LC, Tani K: Molecular abnormality and cDNA cloning of human aldehyde dehydrogenases. Alcohol 2:103-106, 1985

82. Kurys G, Ambroziak W, Pietruszko R: Human aldehyde dehydrogenase. Purification and characterization of a third isozyme with low Km for gamma-aminobutyraldehyde. J Biol Chem 264:4715-4721, 1989

83. Nemet I, Varga-Defterdarovic L, Turk Z: Methylglyoxal in food and living organisms. Mol Nutr Food Res 50:1105-1117, 2006

84. Monder C: Oxidation of methylglyoxal to pyruvic acid by a sheep-liver enzyme. Biochim Biophys Acta 99:573575,1965

85. Vander Jagt DL, Hunsaker LA: Methylglyoxal metabolism and diabetic complications: roles of aldose reductase, glyoxalase-I, betaine aldehyde dehydrogenase and 2-oxoaldehyde dehydrogenase. Chem Biol Interact 143-144:341-351, 2003

86. Ahmed N, Argirov OK, Minhas HS, Cordeiro CA, Thornalley PJ: Assay of advanced glycation endproducts (AGEs): surveying AGEs by chromatographic assay with derivatization by 6-aminoquinolyl-N-hydroxysuccinimidylcarbamate and application to Nepsilon-carboxymethyl-lysine- and Nepsilon-(1-carboxyethyl)lysine-modified albumin. Biochem J 364:1-14, 2002

87. Ahmed N, Thornalley PJ: Chromatographic assay of glycation adducts in human serum albumin glycated in vitro by derivatization with 6-aminoquinolyl-N-hydroxysuccinimidyl-carbamate and intrinsic fluorescence. Biochem J 364:15-24, 2002

88. Westwood ME, Thornalley PJ: Molecular characteristics of methylglyoxal-modified bovine and human serum albumins. Comparison with glucose-derived advanced glycation endproduct-modified serum albumins. J Protein Chem 14:359-372, 1995

89. Shipanova IN, Glomb MA, Nagaraj RH: Protein modification by methylglyoxal: chemical nature and synthetic mechanism of a major fluorescent adduct. Arch Biochem Biophys 344:29-36, 1997

90. Oya T, Hattori N, Mizuno Y, Miyata S, Maeda S, Osawa T, Uchida K: Methylglyoxal modification of protein. Chemical and immunochemical characterization of methylglyoxal-arginine adducts. J Biol Chem 274:1849218502, 1999

91. Ahmed MU, Brinkmann Frye E, Degenhardt TP, Thorpe SR, Baynes JW: N-epsilon-(carboxyethyl)lysine, a product of the chemical modification of proteins by methylglyoxal, increases with age in human lens proteins. Biochem J 324 ( Pt 2):565-570, 1997

92. Brinkmann E, Wells-Knecht, K.J., Thorpe, S.R. and Baynes, J.W. : Characterization of an imidazolium compound formed by reaction of methylglyoxal and Na-hippuryllysine. J. Chem. Soc. Perkin Trans. 22:28172818, 1995 
93. Lederer MO, Klaiber RG: Cross-linking of proteins by Maillard processes: characterization and detection of lysine-arginine cross-links derived from glyoxal and methylglyoxal. Bioorg Med Chem 7:2499-2507, 1999

94. Krymkiewicz N: Reactions of methylglyoxal with nucleic acids. FEBS Lett 29:51-54, 1973

95. Vaca CE, Fang JL, Conradi M, Hou SM: Development of a 32P-postlabelling method for the analysis of 2'deoxyguanosine-3'-monophosphate and DNA adducts of methylglyoxal. Carcinogenesis 15:1887-1894, 1994

96. Schneider M, Thoss G, Hubner-Parajsz C, Kientsch-Engel R, Stahl P, Pischetsrieder M: Determination of glycated nucleobases in human urine by a new monoclonal antibody specific for N2-carboxyethyl-2'deoxyguanosine. Chem Res Toxicol 17:1385-1390, 2004

97. Synold T, Xi B, Wuenschell GE, Tamae D, Figarola JL, Rahbar S, Termini J: Advanced glycation end products of DNA: quantification of N2-(1-Carboxyethyl)-2'-deoxyguanosine in biological samples by liquid chromatography electrospray ionization tandem mass spectrometry. Chem Res Toxicol 21:2148-2155, 2008

98. Murata-Kamiya N, Kamiya H, Kaji H, Kasai H: Glyoxal, a major product of DNA oxidation, induces mutations at G:C sites on a shuttle vector plasmid replicated in mammalian cells. Nucleic Acids Res 25:1897-1902, 1997

99. Yamaguchi T, Fujita Y, Kuroki S, Ohtsuka K, Kimoto E: A study on the reaction of human erythrocytes with hydrogen peroxide. J Biochem 94:379-386, 1983

100. Thornalley P, Wolff S, Crabbe J, Stern A: The autoxidation of glyceraldehyde and other simple monosaccharides under physiological conditions catalysed by buffer ions. Biochim Biophys Acta 797:276-287, 1984

101. Thornalley PJ, Stern A: The production of free radicals during the autoxidation of monosaccharides by buffer ions. Carbohydr Res 134:191-204, 1984

102. Okado A, Kawasaki Y, Hasuike Y, Takahashi M, Teshima T, Fujii J, Taniguchi N: Induction of apoptotic cell death by methylglyoxal and 3-deoxyglucosone in macrophage-derived cell lines. Biochem Biophys Res Commun 225:219-224, 1996

103. Chang T, Wang R, Wu L: Methylglyoxal-induced nitric oxide and peroxynitrite production in vascular smooth muscle cells. Free Radic Biol Med 38:286-293, 2005

104. Kikuchi S, Shinpo K, Moriwaka F, Makita Z, Miyata T, Tashiro K: Neurotoxicity of methylglyoxal and 3deoxyglucosone on cultured cortical neurons: synergism between glycation and oxidative stress, possibly involved in neurodegenerative diseases. J Neurosci Res 57:280-289, 1999

105. Schlotterer A, Kukudov G, Bozorgmehr F, Hutter H, Du X, Oikonomou D, Ibrahim Y, Pfisterer F, Rabbani N, Thornalley P, Sayed A, Fleming T, Humpert P, Schwenger V, Zeier M, Hamann A, Stern D, Brownlee M, Bierhaus A, Nawroth $\mathrm{P}$, Morcos M: C. elegans as model for the study of high glucose- mediated life span reduction. Diabetes 58:2450-2456, 2009

106. Dobler D, Ahmed N, Song L, Eboigbodin KE, Thornalley PJ: Increased dicarbonyl metabolism in endothelial cells in hyperglycaemia induces anoikis and impairs angiogenesis by RGD and GFOGER motif modification. Diabetes 55:1961-1969, 2006

107. Duran-Jimenez B, Dobler D, Moffatt S, Rabbani N, Streuli CH, Thornalley PJ, Tomlinson DR, Gardiner NJ: Advanced glycation end products in extracellular matrix proteins contribute to the failure of sensory nerve regeneration in diabetes. Diabetes 58:2893-2903, 2009

108. Esposito C, Gerlach H, Brett J, Stern D, Vlassara H: Endothelial receptor-mediated binding of glucosemodified albumin is associated with increased monolayer permeability and modulation of cell surface coagulant properties. J Exp Med 170:1387-1407, 1989

109. Li YM, Mitsuhashi T, Wojciechowicz D, Shimizu N, Li J, Stitt A, He C, Banerjee D, Vlassara H: Molecular identity and cellular distribution of advanced glycation endproduct receptors: relationship of p60 to OST-48 and p90 to 80K-H membrane proteins. Proc Natl Acad Sci U S A 93:11047-11052, 1996

110. Horiuchi S, Sakamoto Y, Sakai M: Scavenger receptors for oxidized and glycated proteins. Amino Acids 25:283-292, 2003

111. Ohgami N, Nagai R, Ikemoto M, Arai H, Miyazaki A, Hakamata H, Horiuchi S, Nakayama H: CD36, serves as a receptor for advanced glycation endproducts (AGE). J Diabetes Complications 16:56-59, 2002

112. Jono T, Miyazaki A, Nagai R, Sawamura T, Kitamura T, Horiuchi S: Lectin-like oxidized low density lipoprotein receptor-1 (LOX-1) serves as an endothelial receptor for advanced glycation end products (AGE). FEBS Lett 511:170-174, 2002

113. Neeper M, Schmidt AM, Brett J, Yan SD, Wang F, Pan YC, Elliston K, Stern D, Shaw A: Cloning and expression of a cell surface receptor for advanced glycosylation end products of proteins. J Biol Chem 267:14998-15004, 1992 
114. Yan SF, Ramasamy R, Schmidt AM: Receptor for AGE (RAGE) and its ligands-cast into leading roles in diabetes and the inflammatory response. J Mol Med 87:235-247, 2009

115. Bucciarelli LG, Wendt T, Qu W, Lu Y, Lalla E, Rong LL, Goova MT, Moser B, Kislinger T, Lee DC, Kashyap Y, Stern DM, Schmidt AM: RAGE blockade stabilizes established atherosclerosis in diabetic apolipoprotein E-null mice. Circulation 106:2827-2835, 2002

116. Soro-Paavonen A, Watson AM, Li J, Paavonen K, Koitka A, Calkin AC, Barit D, Coughlan MT, Drew BG, Lancaster GI, Thomas M, Forbes JM, Nawroth PP, Bierhaus A, Cooper ME, Jandeleit-Dahm KA: Receptor for advanced glycation end products (RAGE) deficiency attenuates the development of atherosclerosis in diabetes. Diabetes 57:2461-2469, 2008

117. Goova MT, Li J, Kislinger T, Qu W, Lu Y, Bucciarelli LG, Nowygrod S, Wolf BM, Caliste X, Yan SF, Stern DM, Schmidt AM: Blockade of receptor for advanced glycation end-products restores effective wound healing in diabetic mice. Am J Pathol 159:513-525, 2001

118. Wendt TM, Tanji N, Guo J, Kislinger TR, Qu W, Lu Y, Bucciarelli LG, Rong LL, Moser B, Markowitz GS, Stein G, Bierhaus A, Liliensiek B, Arnold B, Nawroth PP, Stern DM, D'Agati VD, Schmidt AM: RAGE drives the development of glomerulosclerosis and implicates podocyte activation in the pathogenesis of diabetic nephropathy. Am $J$ Pathol 162:1123-1137, 2003

119. Barile GR, Pachydaki SI, Tari SR, Lee SE, Donmoyer CM, Ma W, Rong LL, Buciarelli LG, Wendt T, Horig H, Hudson BI, Qu W, Weinberg AD, Yan SF, Schmidt AM: The RAGE axis in early diabetic retinopathy. Invest Ophthalmol Vis Sci 46:2916-2924, 2005

120. Yan SF, Ramasamy R, Schmidt AM: The RAGE axis: a fundamental mechanism signaling danger to the vulnerable vasculature. Circ Res 106:842-853, 2010

121. Boulanger E, Wautier MP, Wautier JL, Boval B, Panis Y, Wernert N, Danze PM, Dequiedt P: AGEs bind to mesothelial cells via RAGE and stimulate VCAM-1 expression. Kidney Int 61:148-156, 2002

122. Kislinger T, Fu C, Huber B, Qu W, Taguchi A, Du Yan S, Hofmann M, Yan SF, Pischetsrieder M, Stern D, Schmidt AM: N(epsilon)-(carboxymethyl)lysine adducts of proteins are ligands for receptor for advanced glycation end products that activate cell signaling pathways and modulate gene expression. J Biol Chem 274:31740-31749, 1999

123. Kunt T, Forst T, Harzer O, Buchert G, Pfutzner A, Lobig M, Zschabitz A, Stofft E, Engelbach M, Beyer J: The influence of advanced glycation endproducts (AGE) on the expression of human endothelial adhesion molecules. Exp Clin Endocrinol Diabetes 106:183-188, 1998

124. Westwood ME, Thornalley PJ: Induction of synthesis and secretion of interleukin 1 beta in the human monocytic THP-1 cells by human serum albumins modified with methylglyoxal and advanced glycation endproducts. Immunol Lett 50:17-21, 1996

125. Abordo EA, Thornalley PJ: Synthesis and secretion of tumour necrosis factor-alpha by human monocytic THP-1 cells and chemotaxis induced by human serum albumin derivatives modified with methylglyoxal and glucose-derived advanced glycation endproducts. Immunol Lett 58:139-147, 1997

126. Abordo EA, Westwood ME, Thornalley PJ: Synthesis and secretion of macrophage colony stimulating factor by mature human monocytes and human monocytic THP-1 cells induced by human serum albumin derivatives modified with methylglyoxal and glucose-derived advanced glycation endproducts. Immunol Lett 53:7-13, 1996

127. Lieuw-a-Fa ML, Schalkwijk CG, Engelse M, van Hinsbergh VW: Interaction of Nepsilon(carboxymethyl)lysineand methylglyoxal-modified albumin with endothelial cells and macrophages. Splice variants of RAGE may limit the responsiveness of human endothelial cells to AGEs. Thromb Haemost 95:320-328, 2006

128. Giardino I, Edelstein D, Brownlee M: Nonenzymatic glycosylation in vitro and in bovine endothelial cells alters basic fibroblast growth factor activity. A model for intracellular glycosylation in diabetes. J Clin Invest 94:110-117, 1994

129. Schalkwijk CG, van Bezu J, van der Schors RC, Uchida K, Stehouwer CD, van Hinsbergh VW: Heat-shock protein 27 is a major methylglyoxal-modified protein in endothelial cells. FEBS Lett 580:1565-1570, 2006

130. Sakamoto H, Mashima T, Yamamoto K, Tsuruo T: Modulation of heat-shock protein 27 (Hsp27) antiapoptotic activity by methylglyoxal modification. J Biol Chem 277:45770-45775, 2002

131. Yao D, Taguchi T, Matsumura T, Pestell R, Edelstein D, Giardino I, Suske G, Rabbani N, Thornalley PJ, Sarthy VP, Hammes HP, Brownlee M: High glucose increases angiopoietin-2 transcription in microvascular endothelial cells through methylglyoxal modification of mSin3A. J Biol Chem 282:31038-31045, 2007

132. Thangarajah H, Yao D, Chang El, Shi Y, Jazayeri L, Vial IN, Galiano RD, Du XL, Grogan R, Galvez MG, Januszyk M, Brownlee M, Gurtner GC: The molecular basis for impaired hypoxia-induced VEGF expression in diabetic tissues. Proc Natl Acad Sci U S A 106:13505-13510, 2009 
133. Bucala R, Tracey KJ, Cerami A: Advanced glycosylation products quench nitric oxide and mediate defective endothelium-dependent vasodilatation in experimental diabetes. J Clin Invest 87:432-438, 1991

134. Hogan M, Cerami A, Bucala R: Advanced glycosylation endproducts block the antiproliferative effect of nitric oxide. Role in the vascular and renal complications of diabetes mellitus. J Clin Invest 90:1110-1115, 1992

135. Queisser MA, Yao D, Geisler S, Hammes HP, Lochnit G, Schleicher ED, Brownlee M, Preissner KT: Hyperglycaemia impairs proteasome function by methylglyoxal. Diabetes 59:670-678, 2009

136. Brownlee M, Vlassara H, Kooney A, Ulrich P, Cerami A: Aminoguanidine prevents diabetes-induced arterial wall protein cross-linking. Science 232:1629-1632, 1986

137. Soulis T, Cooper ME, Vranes D, Bucala R, Jerums G: Effects of aminoguanidine in preventing experimental diabetic nephropathy are related to the duration of treatment. Kidney Int 50:627-634, 1996

138. Kihara M, Schmelzer JD, Poduslo JF, Curran GL, Nickander KK, Low PA: Aminoguanidine effects on nerve blood flow, vascular permeability, electrophysiology, and oxygen free radicals. Proc Natl Acad Sci U S A 88:61076111, 1991

139. Kazachkov M, Chen K, Babiy S, Yu PH: Evidence for in vivo scavenging by aminoguanidine of formaldehyde produced via semicarbazide-sensitive amine oxidase-mediated deamination. J Pharmacol Exp Ther 322:12011207, 2007

140. Corbett JA, Tilton RG, Chang K, Hasan KS, Ido Y, Wang JL, Sweetland MA, Lancaster JR, Jr., Williamson JR, McDaniel ML: Aminoguanidine, a novel inhibitor of nitric oxide formation, prevents diabetic vascular dysfunction. Diabetes 41:552-556, 1992

141. Yu PH, Zuo DM: Aminoguanidine inhibits semicarbazide-sensitive amine oxidase activity: implications for advanced glycation and diabetic complications. Diabetologia 40:1243-1250, 1997

142. Giardino I, Fard AK, Hatchell DL, Brownlee M: Aminoguanidine inhibits reactive oxygen species formation, lipid peroxidation, and oxidant-induced apoptosis. Diabetes 47:1114-1120, 1998

143. Bolton WK, Cattran DC, Williams ME, Adler SG, Appel GB, Cartwright K, Foiles PG, Freedman BI, Raskin P, Ratner RE, Spinowitz BS, Whittier FC, Wuerth JP: Randomized trial of an inhibitor of formation of advanced glycation end products in diabetic nephropathy. Am J Nephrol 24:32-40, 2004

144. Freedman BI, Wuerth JP, Cartwright K, Bain RP, Dippe S, Hershon K, Mooradian AD, Spinowitz BS: Design and baseline characteristics for the aminoguanidine Clinical Trial in Overt Type 2 Diabetic Nephropathy (ACTION II). Control Clin Trials 20:493-510, 1999

145. Vasan S, Zhang X, Kapurniotu A, Bernhagen J, Teichberg S, Basgen J, Wagle D, Shih D, Terlecky I, Bucala R, Cerami A, Egan J, Ulrich P: An agent cleaving glucose-derived protein crosslinks in vitro and in vivo. Nature 382:275-278, 1996

146. Zieman SJ, Melenovsky V, Clattenburg L, Corretti MC, Capriotti A, Gerstenblith G, Kass DA: Advanced glycation endproduct crosslink breaker (alagebrium) improves endothelial function in patients with isolated systolic hypertension. J Hypertens 25:577-583, 2007

147. Nobecourt E, Zeng J, Davies MJ, Brown BE, Yadav S, Barter PJ, Rye KA: Effects of cross-link breakers, glycation inhibitors and insulin sensitisers on HDL function and the non-enzymatic glycation of apolipoprotein A-I. Diabetologia 51:1008-1017, 2008

148. Giannarelli R, Aragona M, Coppelli A, Del Prato S: Reducing insulin resistance with metformin: the evidence today. Diabetes Metab 29:6S28-35, 2003

149. Effect of intensive blood-glucose control with metformin on complications in overweight patients with type 2 diabetes (UKPDS 34). UK Prospective Diabetes Study (UKPDS) Group. Lancet 352:854-865, 1998

150. Ruggiero-Lopez D, Lecomte M, Moinet G, Patereau G, Lagarde M, Wiernsperger N: Reaction of metformin with dicarbonyl compounds. Possible implication in the inhibition of advanced glycation end product formation. Biochem Pharmacol 58:1765-1773, 1999

151. Beisswenger PJ, Howell SK, Touchette AD, Lal S, Szwergold BS: Metformin reduces systemic methylglyoxal levels in type 2 diabetes. Diabetes 48:198-202, 1999

152. Furchgott RF, Vanhoutte PM: Endothelium-derived relaxing and contracting factors. FASEB J 3:2007-2018, 1989

153. Vanhoutte PM: How We Learned to Say NO. Arterioscler Thromb Vasc Biol 29:1156-1160, 2009

154. Goldin A, Beckman JA, Schmidt AM, Creager MA: Advanced glycation end products: sparking the development of diabetic vascular injury. Circulation 114:597-605, 2006

155. Le Brocq M, Leslie SJ, Milliken P, Megson IL: Endothelial dysfunction: from molecular mechanisms to measurement, clinical implications, and therapeutic opportunities. Antioxid Redox Signal 10:1631-1674, 2008 
156. Patel S, Celermajer DS: Assessment of vascular disease using arterial flow mediated dilatation. Pharmacol Rep 58 Suppl:3-7, 2006

157. Deanfield JE, Halcox JP, Rabelink TJ: Endothelial function and dysfunction: testing and clinical relevance. Circulation 115:1285-1295, 2007

158. Steinberg HO, Chaker H, Leaming R, Johnson A, Brechtel G, Baron AD: Obesity/insulin resistance is associated with endothelial dysfunction. Implications for the syndrome of insulin resistance. J Clin Invest 97:2601-2610, 1996

159. Makimattila S, Virkamaki A, Groop PH, Cockcroft J, Utriainen T, Fagerudd J, Yki-Jarvinen H: Chronic hyperglycaemia impairs endothelial function and insulin sensitivity via different mechanisms in insulindependent diabetes mellitus. Circulation 94:1276-1282, 1996

160. Diederich D, Skopec J, Diederich A, Dai FX: Endothelial dysfunction in mesenteric resistance arteries of diabetic rats: role of free radicals. Am J Physiol 266:H1153-1161, 1994

161. Pflueger AC, Osswald H, Knox FG: Adenosine-induced renal vasoconstriction in diabetes mellitus rats: role of nitric oxide. Am J Physiol 276:F340-346, 1999

162. Constans J, Conri C: Circulating markers of endothelial function in cardiovascular disease. Clin Chim Acta 368:33-47, 2006

163. Cernes R, Zimlichman R, Shargorodsky M: Arterial elasticity in cardiovascular disease: focus on hypertension, metabolic syndrome and diabetes. Adv Cardiol 45:65-81, 2008

164. Simon A, Gariepy J, Chironi G, Megnien JL, Levenson J: Intima-media thickness: a new tool for diagnosis and treatment of cardiovascular risk. J Hypertens 20:159-169, 2002

165. Cines DB, Pollak ES, Buck CA, Loscalzo J, Zimmerman GA, McEver RP, Pober JS, Wick TM, Konkle BA, Schwartz BS, Barnathan ES, McCrae KR, Hug BA, Schmidt AM, Stern DM: Endothelial cells in physiology and in the pathophysiology of vascular disorders. Blood 91:3527-3561, 1998

166. Schalkwijk CG, Stehouwer CD: Vascular complications in diabetes mellitus: the role of endothelial dysfunction. Clin Sci (Lond) 109:143-159, 2005

167. Stehouwer CD, Lambert J, Donker AJ, van Hinsbergh VW: Endothelial dysfunction and pathogenesis of diabetic angiopathy. Cardiovasc Res 34:55-68, 1997

168. Vervoort G, Lutterman JA, Smits P, Berden JH, Wetzels JF: Transcapillary escape rate of albumin is increased and related to haemodynamic changes in normo-albuminuric type 1 diabetic patients. J Hypertens 17:1911-1916, 1999

169. Schalkwijk CG, Poland DC, van Dijk W, Kok A, Emeis JJ, Drager AM, Doni A, van Hinsbergh VW, Stehouwer $\mathrm{CD}$ : Plasma concentration of C-reactive protein is increased in type I diabetic patients without clinical macroangiopathy and correlates with markers of endothelial dysfunction: evidence for chronic inflammation. Diabetologia 42:351-357, 1999

170. van Ittersum FJ, Spek JJ, Praet IJ, Lambert J, RG IJ, Fischer HR, Nikkels RE, Van Bortel LM, Donker AJ, Stehouwer CD: Ambulatory blood pressures and autonomic nervous function in normoalbuminuric type I diabetic patients. Nephrol Dial Transplant 13:326-332, 1998

171. Kilhovd BK, Berg TJ, Birkeland KI, Thorsby P, Hanssen KF: Serum levels of advanced glycation end products are increased in patients with type 2 diabetes and coronary heart disease. Diabetes Care 22:1543-1548, 1999

172. Stitt AW, He C, Friedman S, Scher L, Rossi P, Ong L, Founds H, Li YM, Bucala R, Vlassara H: Elevated AGEmodified ApoB in sera of euglycemic, normolipidemic patients with atherosclerosis: relationship to tissue AGEs. Mol Med 3:617-627, 1997

173. Vlassara H: Advanced glycation end-products and atherosclerosis. Ann Med 28:419-426, 1996

174. Meng J, Sakata N, Takebayashi S, Asano T, Futata T, Nagai R, Ikeda K, Horiuchi S, Myint T, Taniguchi N: Glycoxidation in aortic collagen from STZ-induced diabetic rats and its relevance to vascular damage. Atherosclerosis 136:355-365, 1998

175. Bucala R, Mitchell R, Arnold K, Innerarity T, Vlassara H, Cerami A: Identification of the major site of apolipoprotein $\mathrm{B}$ modification by advanced glycosylation end products blocking uptake by the low density lipoprotein receptor. J Biol Chem 270:10828-10832, 1995

176. Klein RL, Laimins M, Lopes-Virella MF: Isolation, characterization, and metabolism of the glycated and nonglycated subfractions of low-density lipoproteins isolated from type I diabetic patients and nondiabetic subjects. Diabetes 44:1093-1098, 1995

177. Sakata N, Meng J, Takebayashi S: Effects of advanced glycation end products on the proliferation and fibronectin production of smooth muscle cells. J Atheroscler Thromb 7:169-176, 2000 
178. Park L, Raman KG, Lee KJ, Lu Y, Ferran L, Jr., Chow WS, Stern D, Schmidt AM: Suppression of accelerated diabetic atherosclerosis by the soluble receptor for advanced glycation endproducts. Nat Med 4:1025-1031, 1998 179. Mauer SM, Steffes MW, Ellis EN, Sutherland DE, Brown DM, Goetz FC: Structural-functional relationships in diabetic nephropathy. J Clin Invest 74:1143-1155, 1984

180. Cooper ME: Interaction of metabolic and haemodynamic factors in mediating experimental diabetic nephropathy. Diabetologia 44:1957-1972, 2001

181. Forbes JM, Cooper ME, Oldfield MD, Thomas MC: Role of advanced glycation end products in diabetic nephropathy. J Am Soc Nephrol 14:S254-258, 2003

182. Vlassara H, Striker L, Teichberg S, Fuh H, Li YM, Steffes M: Advanced glycation end products induce glomerular sclerosis and albuminuria in normal rats. Proc Natl Acad Sci U S A 91:11704-11708, 1994

183. Jensen L, Denner L, Schrijvers BF, Tilton RG, Rasch R, Flyvbjerg A: Renal effects of a neutralising RAGEantibody in long-term streptozotocin-diabetic mice. J Endocrinol 188:493-501, 2006

184. Beisswenger PJ, Drummond KS, Nelson RG, Howell SK, Szwergold BS, Mauer M: Susceptibility to diabetic nephropathy is related to dicarbonyl and oxidative stress. Diabetes 54:3274-3281, 2005

185. Klein R, Klein BE, Moss SE: Epidemiology of proliferative diabetic retinopathy. Diabetes Care 15:1875-1891, 1992

186. Klein R, Klein BE, Moss SE, Linton KL: The Beaver Dam Eye Study. Retinopathy in adults with newly discovered and previously diagnosed diabetes mellitus. Ophthalmology 99:58-62, 1992

187. Aiello LP, Gardner TW, King GL, Blankenship G, Cavallerano JD, Ferris FL, 3rd, Klein R: Diabetic retinopathy. Diabetes Care 21:143-156, 1998

188. Engerman RL, Kern TS: Progression of incipient diabetic retinopathy during good glycemic control. Diabetes 36:808-812, 1987

189. Su EN, Alder VA, Yu DY, Yu PK, Cringle SJ, Yogesan K: Continued progression of retinopathy despite spontaneous recovery to normoglycemia in a long-term study of streptozotocin-induced diabetes in rats. Graefes Arch Clin Exp Ophthalmol 238:163-173, 2000

190. Hammes HP, Alt A, Niwa T, Clausen JT, Bretzel RG, Brownlee M, Schleicher ED: Differential accumulation of advanced glycation end products in the course of diabetic retinopathy. Diabetologia 42:728-736, 1999

191. Murata T, Nagai R, Ishibashi T, Inomuta H, Ikeda K, Horiuchi S: The relationship between accumulation of advanced glycation end products and expression of vascular endothelial growth factor in human diabetic retinas. Diabetologia 40:764-769, 1997

192. Schalkwijk CG, Ligtvoet N, Twaalfhoven H, Jager A, Blaauwgeers HG, Schlingemann RO, Tarnow L, Parving $\mathrm{HH}$, Stehouwer CD, van Hinsbergh VW: Amadori albumin in type 1 diabetic patients: correlation with markers of endothelial function, association with diabetic nephropathy, and localization in retinal capillaries. Diabetes 48:2446-2453, 1999

193. Lu M, Kuroki M, Amano S, Tolentino M, Keough K, Kim I, Bucala R, Adamis AP: Advanced glycation end products increase retinal vascular endothelial growth factor expression. J Clin Invest 101:1219-1224, 1998

194. Stitt AW, Bhaduri T, McMullen CB, Gardiner TA, Archer DB: Advanced glycation end products induce bloodretinal barrier dysfunction in normoglycemic rats. Mol Cell Biol Res Commun 3:380-388, 2000

195. Chakravarthy U, Hayes RG, Stitt AW, McAuley E, Archer DB: Constitutive nitric oxide synthase expression in retinal vascular endothelial cells is suppressed by high glucose and advanced glycation end products. Diabetes 47:945-952, 1998

196. Agardh E, Hultberg B, Agardh C: Effects of inhibition of glycation and oxidative stress on the development of cataract and retinal vessel abnormalities in diabetic rats. Curr Eye Res 21:543-549, 2000

197. Gardiner TA, Anderson HR, Stitt AW: Inhibition of advanced glycation end-products protects against retinal capillary basement membrane expansion during long-term diabetes. J Pathol 201:328-333, 2003

198. Brownlee M, Vlassara H, Cerami A: Trapped immunoglobulins on peripheral nerve myelin from patients with diabetes mellitus. Diabetes 35:999-1003, 1986

199. Misur I, Zarkovic K, Barada A, Batelja L, Milicevic Z, Turk Z: Advanced glycation endproducts in peripheral nerve in type 2 diabetes with neuropathy. Acta Diabetol 41:158-166, 2004

200. Ozturk G, Sekeroglu MR, Erdogan E, Ozturk M: The effect of non-enzymatic glycation of extracellular matrix proteins on axonal regeneration in vitro. Acta Neuropathol 112:627-632, 2006

201. Meerwaldt R, Links TP, Graaff R, Hoogenberg K, Lefrandt JD, Baynes JW, Gans RO, Smit AJ: Increased accumulation of skin advanced glycation end-products precedes and correlates with clinical manifestation of diabetic neuropathy. Diabetologia 48:1637-1644, 2005 
202. Cameron NE, Cotter MA, Archibald V, Dines KC, Maxfield EK: Anti-oxidant and pro-oxidant effects on nerve conduction velocity, endoneurial blood flow and oxygen tension in non-diabetic and streptozotocin-diabetic rats. Diabetologia 37:449-459, 1994

203. Bierhaus A, Haslbeck KM, Humpert PM, Liliensiek B, Dehmer T, Morcos M, Sayed AA, Andrassy M, Schiekofer S, Schneider JG, Schulz JB, Heuss D, Neundorfer B, Dierl S, Huber J, Tritschler H, Schmidt AM, Schwaninger M, Haering HU, Schleicher E, Kasper M, Stern DM, Arnold B, Nawroth PP: Loss of pain perception in diabetes is dependent on a receptor of the immunoglobulin superfamily. J Clin Invest 114:1741-1751, 2004

204. Vincent AM, Perrone L, Sullivan KA, Backus C, Sastry AM, Lastoskie C, Feldman EL: Receptor for advanced glycation end products activation injures primary sensory neurons via oxidative stress. Endocrinology 148:548558,2007 
Methylglyoxal and methylglyoxal-arginine adducts do not directly inhibit endothelial nitric oxide synthase

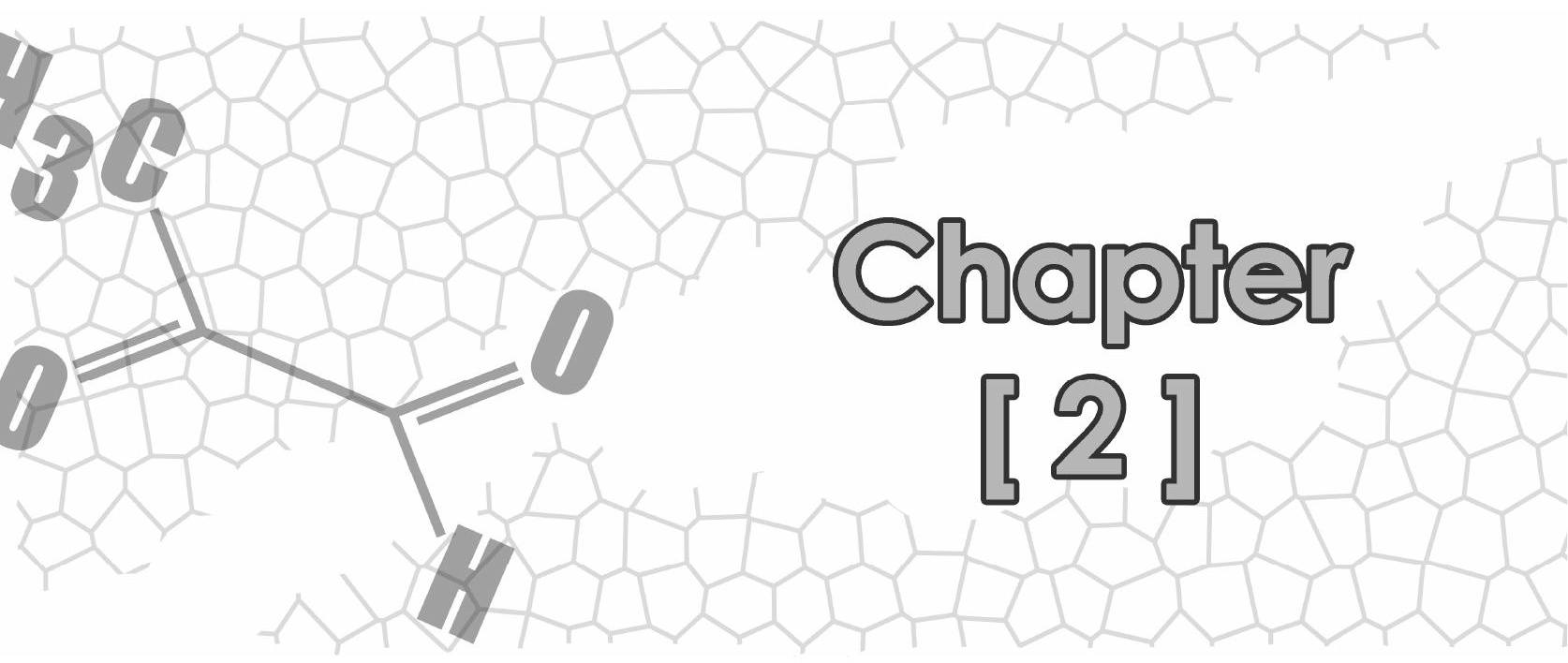

Olaf Brouwers, Tom Teerlink, Jan van Bezu, Rob Barto, Coen Stehouwer and Casper Schalkwijk Ann N Y Acad Sci. 2008 Apr; 1126:231-234. 


\section{Summary}

Increased formation of the reactive dicarbonyl compound methylglyoxal (MGO) and MGOderived advanced glycation end products (AGEs) seems to be implicated in endothelial dysfunction and the development of diabetic vascular complications. MGO reacts with arginine residues in proteins to generate the major glycated adducts $\mathrm{N}_{\delta}$ - $(5$-hydro-5methyl-4-imidazolon-2-yl)-L-ornithine (MG-H1) and arginine-pyrimidine (AP). We investigated whether the free forms of these adducts contribute to vascular cell dysfunction by inhibition of endothelial nitric oxide synthase (eNOS). MG-H1 and AP were synthesized and purified by reversed-phase chromatography, and the conversion of labelled L-arginine to L-citrulline was used to monitor eNOS activity. In contrast to the endogenous eNOS inhibitor asymmetric dimethylarginine (half maximal inhibitory concentration, approximately $5 \mu \mathrm{M}$ ), pathophysiological concentrations of MGO and MG$\mathrm{H} 1$ and AP did not inhibit eNOS activity. Although MGO-derived AGEs are implicated in the development of diabetic vascular complications, this study indicates that this is not mediated via direct inhibition of eNOS activity. 


\section{Introduction}

Vascular disease is the foremost complication of diabetes mellitus, accounting for much excess morbidity and mortality. Endothelial dysfunction is an early event in the pathogenesis of vascular disease, but the mechanism whereby diabetes mellitus leads to endothelial dysfunction is incompletely understood. The accumulation of advanced glycation end products (AGEs) and impaired nitric oxide (NO) metabolism are proposed mechanisms to explain the link between hyperglycaemia and the development of diabetic vascular complications (1). The increase in AGEs and the decrease in NO are closely related to the development and progression of diabetic complications (2).

AGEs are formed from the reaction of reducing sugars with amino acid groups of proteins. AGEs can be formed extracellularly, but it is now apparent that intracellular sugars also participate in AGE formation. In endothelial cells, the highly reactive dicarbonyl compound methylglyoxal (MGO) has been identified as a major precursor in the formation of AGEs (3). MGO is formed non-enzymatically by dephosphorylation of triose phosphates and is efficiently catabolised to D-lactate by the glyoxalase pathway. We recently demonstrated in human endothelial cells that hyperglycaemia produced higher levels of MGO (4). MGO reacts primarily with arginine residues to form arginine-pyrimidine (AP) and $\mathrm{N}_{\delta}$-(5-hydro-5methyl-4-imidazolon-2-yl)-L-ornithine (MG-H1), the latter representing a major AGE in mammalian cells (5). Disturbances in NO metabolism are probably of critical importance in the development of vascular complications in diabetes. The NO synthase reaction involves oxidation of a guanidino nitrogen of L-arginine to NO (6). The synthesis of NO is selectively inhibited by guanidino-substituted analogues of arginine, including endogenously produced asymmetric dimethylarginine (ADMA) (7). Because of the structural homology between NO inhibitors and MGO-arginine adducts (see figure 2.1), we hypothesized that these AGEs are endogenous inhibitors of endothelial nitric oxide synthase (eNOS) activity.

\section{Methods}

\section{Transduction of Human Umbilical Vein Endothelial Cells with eNOS}

Human umbilical vein endothelial cells (HUVECS) were isolated and characterized as described previously (4). For infection with eNOS, $5 \times 10^{5}$ cells, between passages 2-3, were seeded in six-well plates. Twenty-four hours later, cells were washed twice and subsequently infected for 2 hours with increasing amounts of recombinant adenovirus. E1-deleted, replication-deficient, adenoviral vector (Ad5.eNOS) containing $1 \times 10^{12}$ virus particles per $\mathrm{mL}$ were generated on PER.C6 cells as described earlier (8). 


\section{Measurement of eNOS activity}

The kinetics of NO production were assessed in total cell homogenates from Ad5.eNOS transfected cells by measuring the conversion of $\mathrm{L}-\left[{ }^{14} \mathrm{C}\right]$-arginine to $\mathrm{L}-\left[{ }^{14} \mathrm{C}\right]$-citrulline in the presence of the necessary cofactors as previously reported (8). Briefly, cell homogenates of transfected cells were incubated for $60 \mathrm{~min}$ at $37^{\circ} \mathrm{C}$ in $0.1 \mathrm{~mL} 25 \mathrm{mM}$ Tris- $\mathrm{HCl}, \mathrm{pH} 7.4$, supplemented with $3 \mu \mathrm{M}$ tetrahydrobiopterine, $1 \mu \mathrm{M}$ flavin adenine dinucleotide, $100 \mathrm{nM}$ calmoduline, $1 \mu \mathrm{M}$ flavin mono-nucleotide, $1 \mathrm{mM} \mathrm{NADPH}$, and $0.1 \mu \mathrm{Ci} / \mathrm{ml} \mathrm{L}-\left[{ }^{14} \mathrm{C}\right]$-arginine and non-labelled L-arginine. The final concentration of labelled and non-labelled Larginine was $10 \mu \mathrm{M}$. The reaction was started by the addition of $75 \mu \mathrm{M}$ calcium chloride.

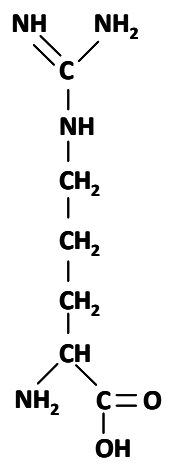

Arginine

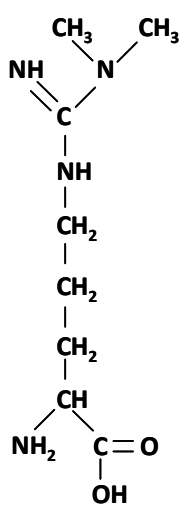

ADMA

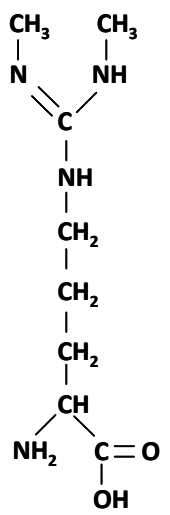

SDMA

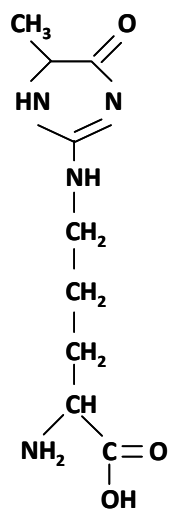

MG-H1

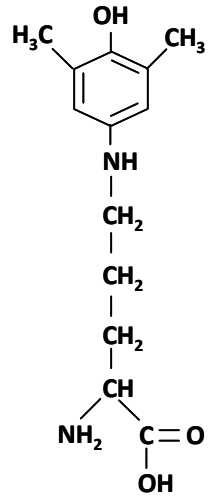

AP

Figure 2.1. Structure of L-Arginine, asymmetric dimethylarginine (ADMA), symmetric dimethylarginine (SDMA), $\mathrm{N}_{\delta}$-(5-hydro-5-methyl-4-imidazolon-2-yl)-L-ornithine (MG-H1), and arginine-pyrimidine (AP). ADMA is known as a nitric oxide synthase inhibitor.

\section{Preparation of methylglyoxal adducts}

AP and MG-H1 were synthesized as described earlier $(5 ; 9)$.

\section{Results}

Under our experimental conditions, the citrulline assay was not sensitive enough for NO measurements in homogenates of non-transfected endothelial cells. We therefore transfected cells with eNOS. Western blot analysis demonstrated a virus-concentrationdependent increase in expression of eNOS protein in Ad5.eNOS-transduced HUVEC that correlated well with activity and reached a plateau value of $\approx 40 \mathrm{pmol}$ citrulline $\min ^{-1} \mathrm{mg}$ protein ${ }^{-1}$ after exposure to a virus concentration of $2 \times 10^{8} \mathrm{pfu} / \mathrm{mL}$ (data not shown). In Ad5.empty-infected cells, NOS activity remained below the detection limit of the assay. The NO production of Ad5.eNOS-transduced cells was calcium-dependent and 
was inhibited by omission of the cofactor $\beta$-NADPH and by L-NG-nitroarginine. These results demonstrate that transduced eNOS is functionally active and can be used to study the effects of inhibitors on eNOS activity.

The ability of free MGO and MGO adducts to inhibit eNOS was investigated using the citrulline formation assay under similar conditions. As shown in figure 2.2, incubation of eNOS with increasing concentrations of MG-H1 led to a slight decrease in eNOS activity at the highest concentration. Also, AP had a mild effect on eNOS activity, but this effect was not dose dependent. Preincubation of eNOS with MGO or MGO adducts did not change the activity of eNOS. Under the same experimental conditions, ADMA, but not symmetric dimethylarginine (SDMA), exhibited a strong concentration-dependent inhibition of eNOS with a half maximal inhibitory concentration $\left(\mathrm{IC}_{50}\right)$ of approximately $5 \mu \mathrm{M}$.

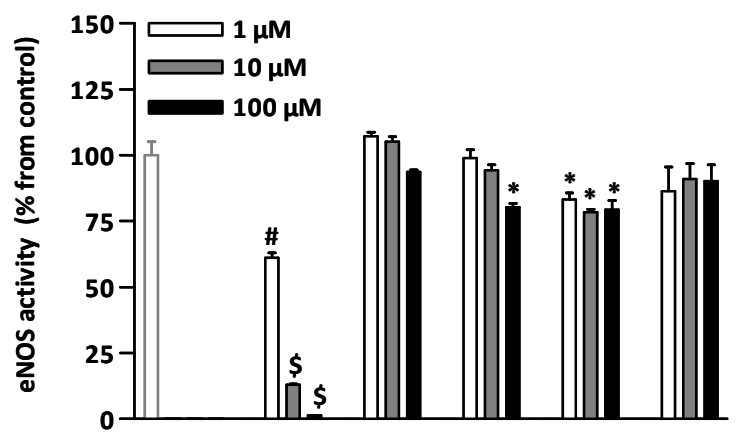

Figure 2.2. The effect of 1,10 and $100 \mu M$ of ADMA, SDMA, MG-H1, AP and free methylglyoxal (MGO) on endothelial nitric oxide synthase (eNOS) activity. The symbols *, \# and $\$$ indicate $p$-value $<0.05, p<0.005$ and $p<$ 0.0005 respectively. Data are expressed as mean \pm standard error of the mean $(n=3)$.

\section{Discussion}

MGO, a reactive dicarbonyl metabolite of glucose, has received considerable attention as the most reactive AGE precursor in endothelial cells (3). Although MGO is involved in a variety of detrimental processes, we found that pathophysiological concentrations of MGO or MGO-arginine adducts do not directly inhibit eNOS activity.

The plasma concentrations of free MGO adducts in the healthy population are around $0.1 \mu \mathrm{M}$ for MG-H1 and $0.05 \mu \mathrm{M}$ for AP. In patients with renal failure these adducts can accumulate to $5 \mu \mathrm{M}$ and $0.1 \mu \mathrm{M}$ respectively (10). In our in vitro system we tested 1$100 \mu \mathrm{M}$ of MG-H1 and AP, but we only saw a mild inhibition of eNOS activity with a 20fold higher concentration $(100 \mu \mathrm{M}$ MG-H1 and $>1 \mu \mathrm{M}$ AP) than pathophysiological concentrations.

Plasma MGO levels are estimated to be around $0.5 \mu \mathrm{M}$ in healthy individuals and can increase to approximately $1 \mu \mathrm{M}$ in diabetic individuals (11). It should be taken into 
account that intracellular levels of MGO are probably higher but are difficult to measure. Nevertheless, in our experiments, even the highest concentration of MGO $(100 \mu \mathrm{M})$ did not show any effect on eNOS activity.

The physiological range of ADMA concentrations in plasma has been determined to be 0.39-0.63 $\mu \mathrm{M}$ and in several pathophysiological situations the ADMA levels can increase two- to sevenfold $(12 ; 13)$. In our study, the $I_{50}$ of ADMA was approximately $5 \mu \mathrm{M}$, which is in the same range as previously reported (14).

MGO and AGEs are also a source of oxidative stress, which can lead to ADMA accumulation by inhibition of dimethylaminohydrolases (DDAH), enzymes that selectively degrade ADMA. It is known that hyperglycaemia leads to lower DDAH activity and thereby to ADMA accumulation (15). Indeed, ADMA was shown to be elevated in streptozotocininduced diabetic rats and could be inhibited by glycaemic control (16). Furthermore, Yin et al. reported that glycated albumin is able to inhibit DDAH activity, thereby impairing NO synthesis. This impairment could be restored by pravastatin and the antioxidant PDTC (17). In line with this, we cannot exclude that the MGO adducts indirectly cause inhibition of eNOS.

We demonstrated that pathophysiological levels of free MGO and the MGO adduct MG$\mathrm{H} 1$ and AP do not directly inhibit eNOS activity like ADMA does. We conclude that MGO and MGO-derived AGEs are implicated in the development of diabetic vascular complications, but that this is not mediated via direct inhibition of eNOS activity. 


\section{References}

1. Bucala R, Tracey KJ, Cerami A: Advanced glycosylation products quench nitric oxide and mediate defective endothelium-dependent vasodilatation in experimental diabetes. J Clin Invest 87:432-438, 1991

2. Tan KC, Chow WS, Ai VH, Metz C, Bucala R, Lam KS: Advanced glycation end products and endothelial dysfunction in type 2 diabetes. Diabetes Care 25:1055-1059, 2002

3. Shinohara M, Thornalley PJ, Giardino I, Beisswenger P, Thorpe SR, Onorato J, Brownlee M: Overexpression of glyoxalase-I in bovine endothelial cells inhibits intracellular advanced glycation endproduct formation and prevents hyperglycaemia-induced increases in macromolecular endocytosis. J Clin Invest 101:1142-1147, 1998

4. Schalkwijk CG, van Bezu J, van der Schors RC, Uchida K, Stehouwer CD, van Hinsbergh VW: Heat-shock protein 27 is a major methylglyoxal-modified protein in endothelial cells. FEBS Lett 580:1565-1570, 2006

5. Ahmed N, Argirov OK, Minhas HS, Cordeiro CA, Thornalley PJ: Assay of advanced glycation endproducts (AGEs): surveying AGEs by chromatographic assay with derivatization by 6 -aminoquinolyl-N-hydroxysuccinimidylcarbamate and application to Nepsilon-carboxymethyl-lysine- and Nepsilon-(1-carboxyethyl)lysine-modified albumin. Biochem J 364:1-14, 2002

6. Huang PL, Huang Z, Mashimo H, Bloch KD, Moskowitz MA, Bevan JA, Fishman MC: Hypertension in mice lacking the gene for endothelial nitric oxide synthase. Nature 377:239-242, 1995

7. Vallance P, Leone A, Calver A, Collier J, Moncada S: Accumulation of an endogenous inhibitor of nitric oxide synthesis in chronic renal failure. Lancet 339:572-575, 1992

8. Havenga MJ, van Dam B, Groot BS, Grimbergen JM, Valerio D, Bout A, Quax PH: Simultaneous detection of NOS-3 protein expression and nitric oxide production using a flow cytometer. Anal Biochem 290:283-291, 2001

9. Oya T, Hattori N, Mizuno Y, Miyata S, Maeda S, Osawa T, Uchida K: Methylglyoxal modification of protein. Chemical and immunochemical characterization of methylglyoxal-arginine adducts. J Biol Chem 274:1849218502, 1999

10. Thornalley PJ, Battah S, Ahmed N, Karachalias N, Agalou S, Babaei-Jadidi R, Dawnay A: Quantitative screening of advanced glycation endproducts in cellular and extracellular proteins by tandem mass spectrometry. Biochem J 375:581-592, 2003

11. Lapolla A, Flamini R, Dalla Vedova A, Senesi A, Reitano R, Fedele D, Basso E, Seraglia R, Traldi P: Glyoxal and methylglyoxal levels in diabetic patients: quantitative determination by a new GC/MS method. Clin Chem Lab Med 41:1166-1173, 2003

12. Horowitz JD, Heresztyn T: An overview of plasma concentrations of asymmetric dimethylarginine (ADMA) in health and disease and in clinical studies: methodological considerations. J Chromatogr B Analyt Technol Biomed Life Sci 851:42-50, 2007

13. Teerlink T: HPLC analysis of ADMA and other methylated L-arginine analogs in biological fluids. J Chromatogr B Analyt Technol Biomed Life Sci 851:21-29, 2007

14. Tsikas D, Boger RH, Sandmann J, Bode-Boger SM, Frolich JC: Endogenous nitric oxide synthase inhibitors are responsible for the L-arginine paradox. FEBS Lett 478:1-3, 2000

15. Noyman I, Marikovsky M, Sasson S, Stark AH, Bernath K, Seger R, Madar Z: Hyperglycaemia reduces nitric oxide synthase and glycogen synthase activity in endothelial cells. Nitric Oxide 7:187-193, 2002

16. Xiong Y, Fu YF, Fu SH, Zhou HH: Elevated levels of the serum endogenous inhibitor of nitric oxide synthase and metabolic control in rats with streptozotocin-induced diabetes. J Cardiovasc Pharmacol 42:191-196, 2003

17. Yin QF, Xiong Y: Pravastatin restores DDAH activity and endothelium-dependent relaxation of rat aorta after exposure to glycated protein. J Cardiovasc Pharmacol 45:525-532, 2005 
Chapter 2 
Hyperglycaemia-induced impaired endothelium-dependent vasorelaxation in rat mesenteric arteries is mediated by intracellular methylglyoxal levels in a pathway dependent on oxidative stress

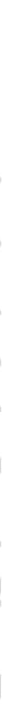

Olaf Brouwers, Petra Niessen, Guido Haenen, Toshio Miyata, Michael Brownlee, Coen Stehouwer, Jo De Mey and Casper Schalkwijk Diabetologia. 2010 May; 53(5):989-1000. 


\section{Summary}

Impaired nitric oxide (NO)-dependent vasorelaxation plays a key role in the development of diabetic vascular complications. We now investigated the effect of hyperglycaemia on impaired vasoreactivity and a putative role of the advanced glycation endproducts (AGE) precursor methylglyoxal (MGO) therein.

The effects of high glucose and MGO on NO-dependent vasorelaxation in isolated rat mesenteric arteries from wild type and transgenic glyoxalase-I (GLO-I) rats, i.e. the enzyme detoxifying MGO, were recorded in a wire myograph. AGE formation of the major MGOadduct 5-hydro-5-methylimidazolone (MG-H1) was detected with an antibody against MG$\mathrm{H} 1$ and quantified with LC-MSMS. Reactive oxygen species (ROS) formation was measured with a $\mathrm{CM}-\mathrm{H}_{2}$ DCFDA probe and by immunohistochemistry with an antibody against nitrotyrosine.

High glucose and MGO exposure of mesenteric arteries significantly reduced the efficacy of NO-dependent vasorelaxation $(p<0.05)$. This impairment was not observed in mesenteric arteries of GLO-I transgenic rats indicating a specific intracellular MGO effect. The diabetes-induced impaired potency $\left(\mathrm{pD}_{2}\right)$ in mesenteric arteries of wild type rats was significantly improved by GLO-I overexpression ( $p<0.05$ ). MGO-modified albumin did not affect NO-dependent vasorelaxation, while under the same conditions the receptor for AGE ligand S100b did ( $p<0.05)$. MGO treatment of arteries increased intracellular staining of MG-H1 in endothelial cells and adventitia by 5 -fold accompanied by an increase in the oxidative stress marker nitrotyrosine by 8 -fold. Antioxidant pre-incubation prevented MGO-induced impaired vasoreactivity.

These data show that hyperglycaemia-induced impaired endothelium-dependent vasorelaxation is mediated by increased intracellular MGO levels in an oxidative stressdependent pathway. 


\section{Introduction}

Diabetes mellitus is associated with an increased risk for cardiovascular disease. Endothelial dysfunction is considered to be the primary cause in the pathogenesis of vascular disease in diabetes. Although the mechanism whereby diabetes leads to endothelial dysfunction is incompletely understood, a common feature of endothelial dysfunction is a decreased bioavailability of endothelium-derived nitric oxide (NO) and, as a consequence, impaired endothelium-dependent vasorelaxation. Several studies demonstrated impaired endothelium-dependent vasorelaxation in diabetic patients $(1 ; 2)$ and in animal models of diabetes $(3 ; 4)$.

One hypothesis about how hyperglycaemia leads to endothelial dysfunction and vascular complications is the formation of advanced glycation endproducts (AGEs) (5). AGEs are a heterogeneous family of non-enzymatically modified proteins formed by carbohydrates, which are increased in patients with diabetes (6-8). The mechanisms by which AGEs can contribute to vascular complications are multiple, including the interaction of AGEs with the receptor for AGE (RAGE), the formation of cross-links in basal membranes and intracellular accumulation of glycated proteins (5). Although it has been demonstrated that AGEs are associated with an impaired endothelium-dependent vasorelaxation in type 2 diabetes (9) and can reduce endothelium-dependent vasorelaxation (10), it is not known which specific AGEs are the culprits or which pathways are involved.

It is now apparent that especially intracellular sugars and their derivatives can participate in glycation and AGE formation (11). The reactive dicarbonyl methylglyoxal (MGO) has received considerable attention as the most reactive AGE precursor in endothelial cells (12). MGO is formed non-enzymatically by dephosphorylation of triose phosphates and is efficiently catabolised to D-lactate by the glyoxalase pathway, consisting of glyoxalase-I (GLO-I), glyoxalase II and the co-factor glutathione (13). MGO primarily reacts with arginine residues to form the major adduct $\mathrm{N}_{\delta}$-(5-hydro-5-methyl-4-imidazolon-2-yl)-Lornithine (MG-H1), and with lysine to form $\mathrm{N}_{\varepsilon}$-(1-carboxyethyl)lysine (CEL) $(14 ; 15)$. Plasma MGO levels are significantly increased in diabetic patients (16) and in endothelial cells by hyperglycaemia (17). The importance of MGO and MGO-derived AGEs for the development of diabetic vascular complications and hypertension has only recently been recognized. Recent studies demonstrate that increased MGO-derived AGE levels in diabetic patients are associated with diabetic complications such as nephropathy (18) and retinopathy (19) and with diabetes-related vascular disorders such as hypertension (2024). As a major precursor of AGE formation, MGO can influence multiple aspects of cellular biology in diabetes (25). MGO targets specific mitochondrial proteins accompanied by an increase in the formation of reactive oxygen species (ROS) (26). Since ROS production by mitochondria is responsible for major mechanisms involved in diabetic 
complications the MGO-induced ROS production may be an initial event in the pathogenesis of vascular complications.

We hypothesize that accumulation of MGO can lead to endothelial dysfunction by ROS formation. Therefore the present study evaluated the effect of hyperglycaemia, MGO and MGO-modified albumin on endothelium-dependent NO-mediated vasorelaxation, a measure of endothelial function, and tested the involvement of oxidative stress therein.

\section{Methods}

\section{Animals}

All animal studies were carried out in accordance with the guide for the care and use of laboratory animals of the national institutes of health. All experiments involving rats were reviewed and approved by the ethics committee for animal care and use of the Maastricht University, the Netherlands.

GLO-I transgenic rats were obtained from Prof. Dr. T. Miyata (27). Rat genomic DNA extracted from tail tissue was used to detect the transgene by polymerase chain reaction (PCR) using specific primers for GLO-I or pBsCAG-2 vector. Primers for cytomegalovirus enhancer sense (5'-GTC GAC ATT GAT TAT TGA CTA G-3') and antisense (5'-CCA TAA GGT CAT GTA CTG-3'), amplified a $350 \mathrm{bp}$ fragment. Primers for the fragment containing human GLO-I gene and 3' junction of vector, sense (5'-GTA GTG TGG GTG ACT CCT CCG TTC CTT GGG-3') and antisense (5'-TCG AGG GAT CTT CAT AAG AGA AGA G-3'), amplified a $1200 \mathrm{bp}$ fragment. PCR amplification was carried out with an initial denaturation at $95^{\circ} \mathrm{C}$ for five minutes, followed by 35 cycles of $95^{\circ} \mathrm{C}$ for 30 seconds, $55^{\circ} \mathrm{C}$ or $58^{\circ} \mathrm{C}$ for 30 seconds, and $72^{\circ} \mathrm{C}$ for 30 or 75 seconds.

Wild-type rats $(n=9)$ and transgenic GLO-I rats $(n=8)$ were made diabetic by intravenous injection of streptozotocin (STZ, $65 \mathrm{mg} / \mathrm{kg}$ body weight) in the tail vein. Weight and agematched control rats $(n=9)$ and transgenic GLO-I rats $(n=8)$ were not injected. STZ resulted in a 5-fold increase of blood glucose levels, irrespective of the GLO-I overexpression.

\section{MGO modified albumin preparation and characterization}

MGO was purchased from Sigma Aldrich (St. Louis, USA) and MGO-modified albumin was prepared by incubation of human albumin (HSA, Cealb ${ }^{\circledR}$, Sanquin, The Netherlands) in a phosphate buffer $(0.2 \mathrm{M})$, pH 7.8, with 0.5 or $10 \mathrm{mM} \mathrm{MGO}$ for 2, 4 or 8 days. After the incubations, the preparations were extensively dialyzed against PBS at $4^{\circ} \mathrm{C}$ with three changes of solution. The reagents were divided in aliquots and stored at $-20^{\circ} \mathrm{C} . \mathrm{CML}, \mathrm{CEL}$ and MG-H1 were measured by liquid chromatography (LC)-mass spectrometry (MS)/MS as previously described (28). 


\section{Isometric tension measurements}

Healthy adult male wild type Wistar rats, or adult Wistar rats with a human GLO-I overexpression (GLO-I TG) (300 - 450 gram) were sacrificed by $\mathrm{CO}_{2}$ inhalation. Second order mesenteric arteries were isolated and then mounted in a myograph organ bath model (610M Danish Myotechnology by J.P. Trading, Denmark) with two steel $40-\mu \mathrm{m}$ wires inserted through the lumen of the segments. NO-mediated effects were selectively investigated using ACh, (0.01 - $10 \mu \mathrm{M})$ and sodium nitroprusside (SNP, $0.01-10 \mu \mathrm{M}$ ) during contraction with $65 \mathrm{mM} \mathrm{K}^{+}$, which blocks Endothelium Derived Hyperpolarizing Factors (EDHFs), and after preincubation with the prostaglandin blocker indomethacin (10 $\mu \mathrm{M})$ (for detailed methodology see electronic supplementary material, page 63).

\section{Endothelial cell culture}

In the in vitro experiments we used ECRF24 cells which are derived from Human Umbilical Vein Endothelial Cells, immortalized using an amphotrophic, replication-deficient retrovirus containing the E6/E7 genes of human papilloma virus (29). Most of the experiments were also performed with HUVEC and showed similar results as for ECRF24. ECRF24 cells were cultured on fibronectine-coated $75 \mathrm{~cm}^{2}$ flasks and maintained at $37^{\circ} \mathrm{C}$ in $5 \% \mathrm{CO}_{2}$. Culture medium consisted of $\mathrm{M} 199$ (with $\mathrm{Na}_{2} \mathrm{HCO}_{3}$ and glutamine) (Lonza, Basel, Switzerland) containing $20 \%(\mathrm{v} / \mathrm{v}$ ) heat-inactivated fetal calf serum (HyClone, Logan, Utah, USA), $2 \mathrm{mM}$ glutamine (Invitrogen), $100 \mathrm{U} / \mathrm{ml}$ penicillin (Invitrogen, San Diego, USA), 100 $\mu \mathrm{g} / \mathrm{ml}$ streptomycin (Invitrogen), $50 \mu \mathrm{g} / \mathrm{ml}$ endothelial cell growth factor (Sigma Aldrich) and $5 \mathrm{U} / \mathrm{ml}$ heparin (LEO Pharma, The Netherlands).

\section{Immunohistochemistry}

For immunohistochemistry, arteries were fixed with phosphate-buffered $(\mathrm{pH} 7.4)$ formaldehyde (4\%) for 30 minutes at $37^{\circ} \mathrm{C}$ at the end of the myograph experiments. Subsequently, arteries were transferred to $70 \%$ ethanol embedded in paraffin and processed for histological examination. An anti MG-H1 antibody was used for the detection of the major MGO-adduct MG-H1 and a polyclonal nitrotyrosine antibody (Calbiochem, San Diego, USA) was used for the detection of peroxynitrite formation.

\section{Immunofluorescense}

ECRF24 cells were seeded on chamber slides (Lab-Tek II, Nunc, Rochester, USA) fixed with para-formaldehyde (4\%), and permeabilised with triton-X100 (0.1\%). Subsequently mouse anti-MG-H1 IgG (1:5000) was used as the primary antibody and FITC-conjugated antimouse IgG (Dako, Glostrup, Denmark, 1:30) was used as the secondary antibody. Localization was visualized with a Bio-Rad MRC600 confocal microscope (Bio-Rad Laboratories Ltd, Hemel Hempstead, UK) equipped with an air-cooled Argon-Krypton 
mixed gas laser and mounted onto an Axiophote microscope (Zeiss), using oil-immersion objectives (40x, NA=3D1.3 or $63 x, N A=3 D 1.4)$. Cells incubated without the primary antibodies were used to determine non-specific background fluorescence.

\section{Oxidative stress measurement}

Reactive oxygen species were measured with the 5-(and-6-)-chloromethyl-2' $7^{\prime}$ dichlorodihydro-fluorescein diacetate acetyl ester (CM- $\mathrm{H}_{2}$ DCFDA) assay (Invitrogen). In brief, endothelial cells cultured in black 96 wells (Cellstar, Greiner, Monroe, USA) were loaded with $50 \mu \mathrm{M} \mathrm{CM}-\mathrm{H}_{2}$ DCFDA for 45 minutes. Subsequently, cells were incubated with $1.0 \mathrm{mM}$ MGO (with or without $100 \mu \mathrm{M}$ NAC, $10 \mathrm{nM}$ EUK-134 and $10 \mathrm{nM} \mathrm{Mn(III)TMP} \mathrm{pre-}$ treatment) and simultaneously fluorescent signal (485 nm / $516 \mathrm{~nm}$ ) was measured with a micro-plate reader (Synergy, Biotek, Vermont, USA) during 60 minutes. Results are shown as arbitrary units (normalized fluorescent signal produced during 60 minutes) \pm S.E.M.

\section{Glyoxalase-I assay}

Glyoxalase-I activity was assayed by spectrophotometry according to the method of McLellan and Thornalley, monitoring the increase in absorbance at $240 \mathrm{~nm}$ due to the formation of S-D-lactoylglutathione for ten minutes at $25^{\circ} \mathrm{C}(30)$.

\section{Cell viability assay}

To assess cell viability, a 3-[4,5-dimethylthiazol-2-yl]-2,5-diphenyl tetrazolium bromide (MTT) (Invitrogen) assay was used. Briefly, endothelial cells were seeded at a density of $1 \times 10^{4}$ cells per well into 96-well culture plates. Confluent monolayers were then exposed to various MGO concentrations $(0.1 ; 0.33$; and $1.0 \mathrm{mM})$ for 60 minutes. Afterwards cells were incubated with MTT for 3 hours and lysed with hydrogen chloride/isopropanol. Subsequently absorbance was measured at $570 \mathrm{~nm}$ and at $690 \mathrm{~nm}$ for background subtraction.

\section{NO measurement}

Deoxygenated water was spiked with $\bullet$ NO gas and the $\bullet$ NO concentration was monitored with an Iso-NO meter (world Precision Instruments, Sarasota FL, USA) at $37^{\circ} \mathrm{C}$ and the decrease in $\bullet \mathrm{NO}$ concentration was followed in time in the presence or absence of the test compound in solution (for details see electronic supplementary material, page 63).

\section{Statistical analysis}

All values are expressed as mean \pm S.E.M. (shown as vertical bars in the figures). Sensitivity $\left(\mathrm{pD}_{2}\right)$ and maximum response (Emax) was calculated from the concentration-response curves to ACh. pD2 is defined as the negative logarithm to base 10 of the $E_{50}$ values and 
Emax is defined as the maximal vasorelaxation to ACh at the highest concentration used and expressed as 100 minus percentage relative contractions to $65 \mathrm{mM} \mathrm{K}^{+}$. The statistical differences in potency and maximum response between groups was tested using OneWay ANOVA and the shift of the dose response curve was tested with Two-Way ANOVA with a post-hoc Bonferroni correction. A p-value of less than 0.05 was considered statistically significant.

\section{Results}

Hyperglycaemia-induced impaired NO-mediated relaxation in rat mesenteric arteries is normalized by GLO-I overexpression

To investigate the effects of high glucose on vascular reactivity, isolated mesenteric arteries were mounted in a myograph and exposed to 30 and $40 \mathrm{mM}$ glucose for 2 hours. Endothelium-dependent NO-mediated relaxation was tested in these arteries during precontraction with high potassium (65 mM) in the presence of $10 \mu \mathrm{M}$ indomethacin. High glucose did not lead to any alterations in precontraction (active wall tension in response to $65 \mathrm{mM}$ potassium was $2.8 \pm 0.6 \mathrm{~N} / \mathrm{m}$ ). A pre-incubation with 30 or $40 \mathrm{mM}$ glucose reduced ACh-induced vasorelaxation (figure 3.1A). The efficacy significantly decreased (Emax in presence of 5,30 and $40 \mathrm{mM}$ glucose was $67 \pm 2 ; 56 \pm 3$; and $50 \pm 3 \%$ vasorelaxation, respectively; $p<0.01)$, but the potency of $A C h$ was not altered $\left(\mathrm{pD}_{2}\right.$ in presence of 5,30 and $40 \mathrm{mM}$ glucose was $6.6 \pm 0.1 ; 6.7 \pm 0.1$; and $6.5 \pm 0.1$, respectively). This effect of glucose was not due to changes in osmotic values as indicated by normal relaxing responses to the osmotic control mannitol (Emax was $65 \pm 3$ and $\mathrm{pD}_{2} 6.7 \pm 0.1$ ). The effect of high glucose was strictly endothelium-dependent, because there were no alterations in endotheliumindependent relaxation provoked with the NO-donor SNP (figure 3.1B).

To study whether an intracellular accumulation of MGO is involved in high glucoseinduced impaired ACh-induced vasorelaxation, mesenteric arteries of rats with a transgenic overexpression of the cytosolic GLO-I enzyme were used. In these transgenic rats we measured a significant increase $(p<0.001)$ in GLO-I activity in the mesenteric arteries compared with their wild-type littermates (figure 3.1C). GLO-I overexpression prevented high glucose induced impairment of endothelium-dependent NO-mediated relaxation (figure 3.1D), indicating that $M G O$ is involved in high glucose induced impaired vasorelaxation. To test if $\mathrm{MGO}$ is also involved in hyperglycaemia-induced impaired vascular reactivity in vivo, we tested $\mathrm{ACh}$-induced vasorelaxation in the mesenteric arteries of wild type and transgenic GLO-I rats with or without diabetes. Wild type diabetic rats had a decreased vascular reactivity to ACh, (and not to SNP; data not shown) which could be improved by GLO-I overexpression (figure 3.1E). The potency of ACh in the mesenteric arteries was markedly decreased in the wild type diabetic rats, and could be significantly improved by GLO-I overexpression in the transgenic rats (figure 3.1F). 

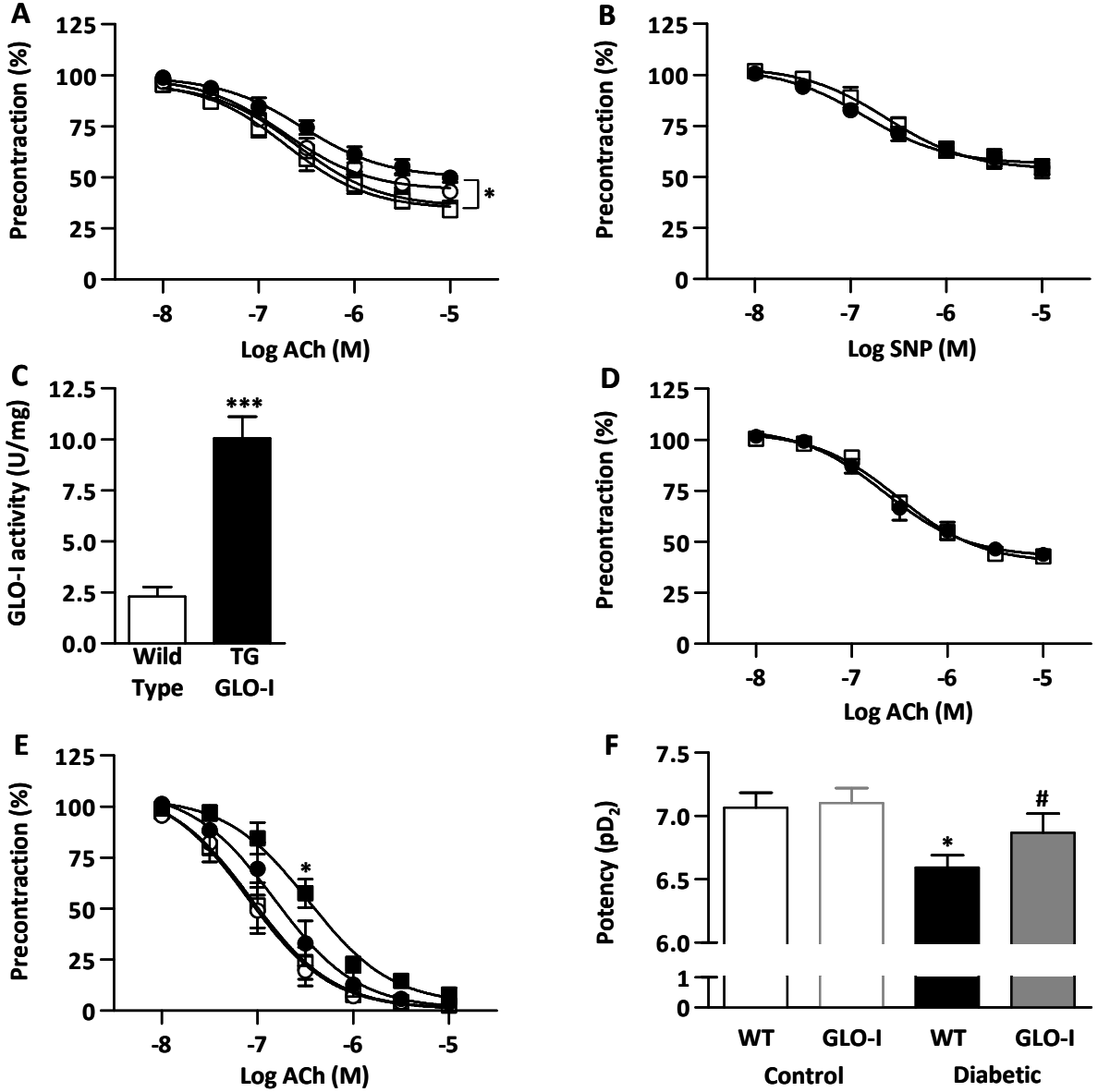

Figure 3.1. Effect of high glucose concentration on acetylcholine-induced NO-mediated endotheliumdependent relaxation in isolated rat mesenteric arteries

A. Rat mesenteric arteries were mounted in a myograph and incubated with or without high glucose concentrations for 2 hours. ACh-induced NO-mediated endothelium-dependent relaxation was impaired in a concentration dependent manner during incubation with $30 \mathrm{mM}$ glucose (white circles) or $40 \mathrm{mM}$ glucose (black circles) compared with control (i.e. $5 \mathrm{mM}$ glucose, white squares). The $40 \mathrm{mM}$ glucose incubation resulted in a statistically significant shift of the dose response curve ( $\# p<0.05$ vs. control group). Incubation with $35 \mathrm{mM}$ mannitol (black squares), as an osmotic control, did not impair NO-mediated endothelium-dependent relaxation $(n=6)$. B. SNP induced NO-mediated endothelium-independent vasorelaxation during incubation without (white squares) or with $40 \mathrm{mM}$ glucose (black circles) for 2 hours did not differ $(n=6)$. C. GLO-I activity in mesenteric arteries homogenates of GLO-I transgenic rats is significantly elevated compared with mesenteric arteries homogenates of wild type rats $(n=4, * * * p<0.001)$. D. In mesenteric arteries of GLO-I transgenic rats, AChinduced NO-mediated endothelium-dependent relaxation is not impaired during incubation with $40 \mathrm{mM}$ glucose (black circles) for 2 hours compared with control incubations (i.e. $5 \mathrm{mM}$ glucose, white squares, $n=5$ ). $E$. AChinduced vasorelaxation was significantly impaired in wild type diabetic rats (black squares; $* p<0.05$ ) as compared with the controls (wild type control; white squares and transgenic control; white circles). GLO-I overexpression improved this relaxation (black circles) $(n=8)$. F. The decreased potency in wild type diabetic rats $(* * \mathrm{p}<0.01)$ was significantly improved by GLO-I overexpression $(\# \mathrm{p}<0.05)$. 


\section{Extracellularly added methylglyoxal-derived AGEs do not lead to impaired vasoreactivity} To test a possible extracellular contribution of MGO-derived AGEs on vascular reactivity, MGO-modified albumin was added to the mesenteric arteries. Minimally- and highlymodified MGO-albumin was prepared by incubating albumin with 0.5 and $10 \mathrm{mM}$ MGO respectively for 2, 4 or 8 days. Concentrations of the AGES CML, CEL and MG-H1 in the 2 days incubated albumin preparations are shown in figure 3.2A. None of these MGOalbumin preparations modified contractile responses or ACh-induced vasorelaxation in rat mesenteric resistance arteries, demonstrating that there is no functional impairment of vasoreactivity due to extracellular MGO-modified albumin adducts (figure 3.2B). Also albumin which was modified with MGO for 4 or 8 days did not affect reactivity compared with control albumin (data not shown). In contrast, under the same experimental conditions, the RAGE ligand S100b significantly $(p<0.01)$ reduced ACh-induced NOmediated vasorelaxation in a dose-dependent manner (figure 3.2C).
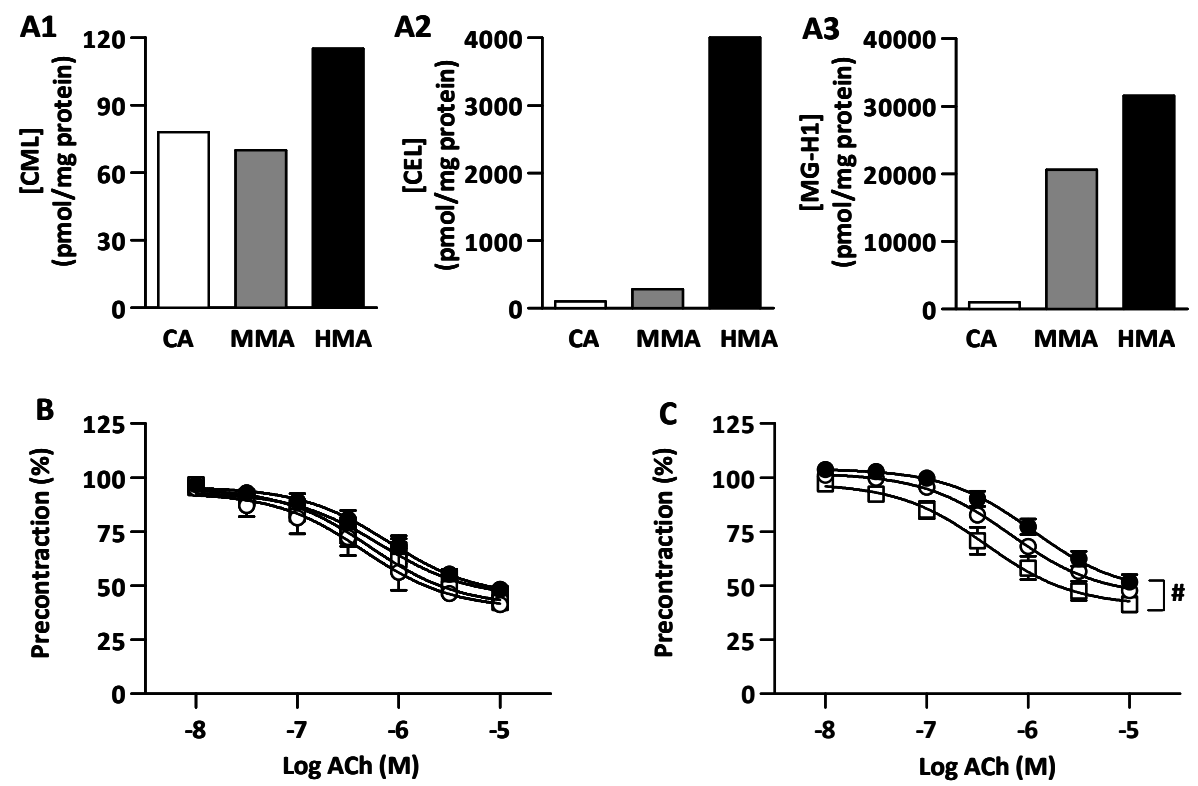

Figure 3.2. Effect of methylglyoxal-modified albumin and S100b on acetylcholine-induced NO-mediated endothelium-dependent relaxation in isolated rat mesenteric arteries

A. Characteristics of MGO-modified albumin. Control albumin (CA) was exposed to MGO (0.5 and 10mM MGO) for 2 days to prepare minimally-modified MGO-albumin (MMA) and highly-modified MGO-albumin (HMA). The amount of $\mathrm{N}_{\varepsilon}$-(carboxymethyl)lysine (CML, A1), $\mathrm{N}_{\varepsilon}$-(carboxyethyl)lysine (CEL, A2) and 5-hydro-5methylimidizalone (MG-H1, A3) was determined with UPLC-MSMS analysis. B. ACh-induced NO-mediated endothelium-dependent relaxation during incubation with albumin (black squares), minimally-modified MGOalbumin (white circles) and highly modified MGO-albumin (black circles) did not statistically differ from the control group (white squares, ( $\mathrm{n=4}$ ). C. Incubation of mesenteric arteries with 20 (white circles) or $40 \mu \mathrm{g} / \mathrm{mL}$ (black circles) of the RAGE ligand S100b resulted in a concentration-dependent impairment of ACh-induced NOmediated endothelium-dependent relaxation. The $40 \mu \mathrm{g} / \mathrm{mL}$ incubation resulted in a statistically significant shift of the dose-response curve ( $\#<<0.05$ vs. control group, $n=4$ ). 


\section{Methylglyoxal leads to impaired endothelium-dependent vasorelaxation}

To determine the direct effect of MGO on vasoreactivity, rat mesenteric resistance arteries were exposed to MGO. The average isometric tension generated by the vessels during precontraction with high potassium (65 mM) was $3.1 \pm 0.5 \mathrm{~N} / \mathrm{m}$, and did not significantly differ between the various incubations. Preincubation of the arteries with MGO significantly reduced ACh-induced vasorelaxation in the presence of $10 \mu \mathrm{M}$ indomethacin in a concentration-dependent manner (figure 3.3A), as indicated by a significantly decreased efficacy (Emax in presence of $0,0.1,0.33$ and $1.0 \mathrm{mM}$ MGO was $62 \pm 3 ; 57 \pm 1 ; 48 \pm 3$ and $39 \pm 3 \%$ vasorelaxation, respectively; $p<0.001$ ). In contrast, the potency of $\mathrm{ACh}$ was not altered $\left(\mathrm{pD}_{2}\right.$ in presence of $0,0.1,0.33$ and $1.0 \mathrm{mM}$ MGO was $6.5 \pm 0.1 ; 6.3 \pm 0.1 ; 6.2 \pm 0.1$ and $6.3 \pm 0.2$, respectively). Longer incubations with lower amounts of MGO (10 and $50 \mu \mathrm{M}$ for 2 hours) also impaired NO-mediated vasorelaxation indicated by decreased efficacy (Emax in presence of 0,10 , and $50 \mu \mathrm{M} \mathrm{MGO}$ was 58 \pm 2 ; $55 \pm 2$ and $46 \pm 3 \%$ vasorelaxation, respectively; $p<0.01$ ) (figure $3.3 \mathrm{~B}$ ). The impairment of ACh-induced vasorelaxation by MGO was endothelium-dependent, as indicated by normal relaxing responses of MGO-pre-incubated arteries to the endothelium-independent NO donor SNP (figure 3.3C).

Exposure of the mesenteric arteries to MGO leads to accumulation of the major MGOderived AGE MG-H1, which was predominantly localized in the adventitia and the endothelium (figure 3.3D, panel a-d).

To confirm the accumulation of MG-H1 by MGO treatment and to further address the localization of MG-H1 in endothelial cells, we incubated endothelial cells with MGO and showed an intracellular localization of MG-H1 in predominantly the cytoplasm and the nucleus of these endothelial cells (figure 3.3D, panel e and f). The MG-H1 accumulation of MGO in mesenteric arteries was concentration-dependent as indicated by quantification of immunostaining of MG-H1 (figure 3.3E) and by UPLC-MSMS (figure 3.3F). These experiments demonstrated that extracellularly added MGO results in intracellular accumulation of MG-H1. 
A

12
100
75
5

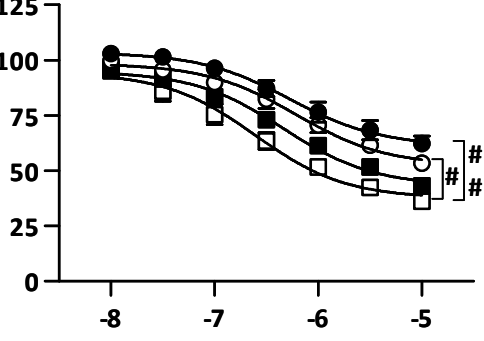

$\log$ ACh (M)
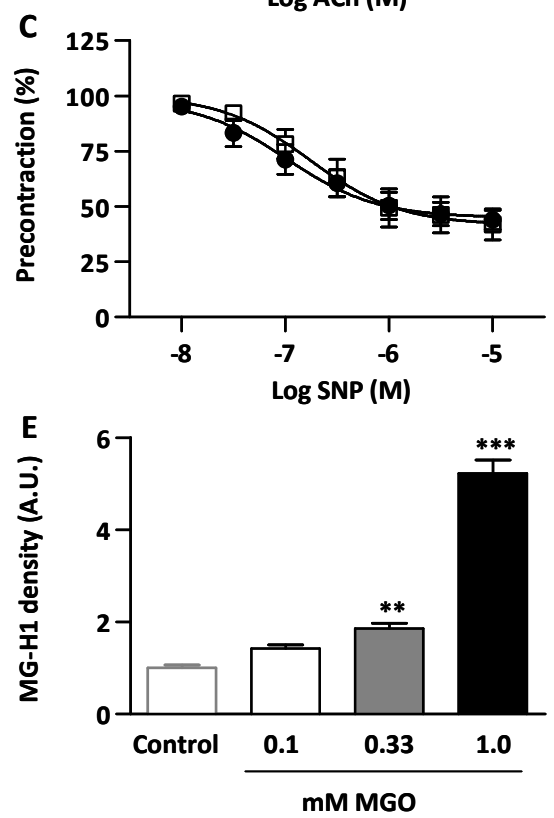

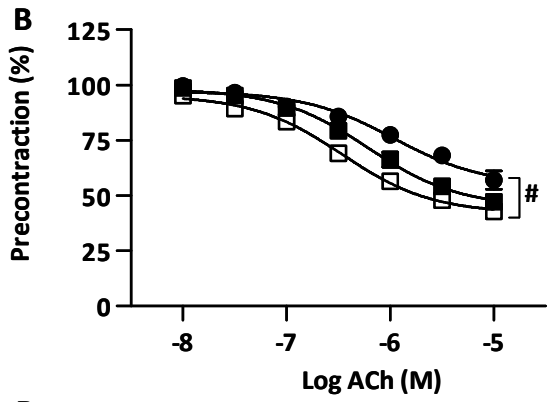

D
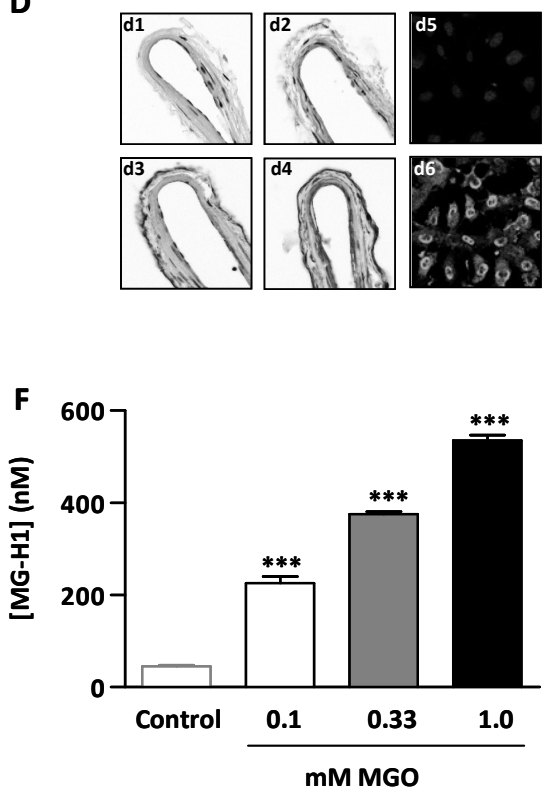

Figure 3.3. Effect of methylglyoxal on vascular reactivity and intracellular MG-H1 formation.

A. Incubation of isolated mesenteric arteries for 1 hour with $0.1 \mathrm{mM}$ MGO (black squares), $0.33 \mathrm{mM}$ MGO (white circles) and $1.0 \mathrm{mM} \mathrm{MGO}$ (black circles) results in impaired ACh-induced NO-mediated endothelium-dependent relaxation compared with the control group (white squares) ( $n=5$ for all conditions). Incubation with $0.33 \mathrm{mM}$ (\# $p<0.05$ vs. control group) and $1.0 \mathrm{mM} \mathrm{MGO}$ (\#\# $\mathrm{p}<0.001$ vs. control group) resulted in a statistically significant shift of the dose-response curve $(n=5)$. B. Incubation of isolated mesenteric arteries for 2 hours with $10 \mu \mathrm{M}$ MGO (black squares) and $50 \mu \mathrm{M} \mathrm{MGO}$ (black circles) results in impaired ACh-induced NO-mediated endotheliumdependent relaxation compared with the control group (white squares) ( $n=4$ for all conditions). Incubation with $50 \mu \mathrm{M}$ MGO resulted in a statistically significant shift of the dose-response curve ( $n=4$ ( $\# p<0.01$ vs. control group)). C. SNP induced NO-mediated endothelium-independent vasorelaxation during incubation without (white squares) or with $1.0 \mathrm{mM} \mathrm{MGO}$ (black circles) for 1 hour did not differ $(n=4)$. D. Localization of MG-H1 in adventitia and endothelial cells of mesenteric arteries not incubated (panel $\mathrm{d} 1$ ) or incubated with $0.1 \mathrm{mM}$ MGO (panel d2), $0.33 \mathrm{mM}$ MGO (panel d3) or $1.0 \mathrm{mM} \mathrm{MGO} \mathrm{(panel} \mathrm{d4)} \mathrm{for} 1$ hour. Furthermore intracellular localization of MG-H1 was determined in cultured endothelial cells (ECRF24) incubated without (panel d5) or with $1.0 \mathrm{mM} \mathrm{MGO}$ (panel d6) for 1 hour by confocal laser scanning microscopy (colour figures on page 158). E. Quantification of MG-H1 staining in MGO exposed arteries ( $n=4)$. F. Concentration-dependent increase of MG-H1 levels in protein lysates of mesenteric arteries not incubated or incubated with $0.1,0.33$ or $1.0 \mathrm{mM}$ MGO for 1 hour as determined by UPLC-MSMS $(n=3) .{ }^{* *} p<0.01$ vs. control group. ${ }^{* * *} p<0.001$ vs. control group. 
Intracellular effects of methylglyoxal contribute to methylglyoxal-induced impaired endothelium-dependent vasorelaxation

To study whether the intracellular accumulation of MGO and/or MG-H1 is involved in MGO-induced impaired ACh-induced vasorelaxation, arteries of GLO-I transgenic rats were incubated with $1.0 \mathrm{mM}$ MGO. These arteries did not show elevated MG-H1 levels while MGO-incubated arteries of wild type rats did (figure 3.4A). Furthermore the impaired vasoreactivity of the mesenteric arteries of MGO-incubated rats was normalized by GLO-I overexpression (figure 3.4B), indicating that extracellularly added MGO leads to intracellular processes which contribute to MGO-impaired endothelium-dependent vasorelaxation.

A

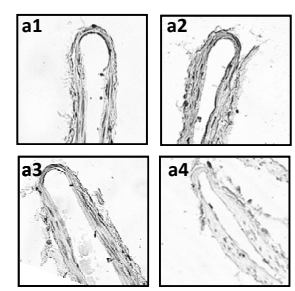

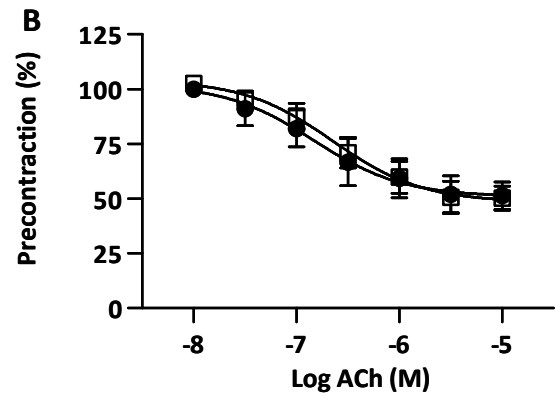

Figure 3.4. Effect of glyoxalase-I overexpression on the methylglyoxal-induced formation of the major methylglyoxal-adduct, 5-hydro-5-methylimidazolone (MG-H1) and on vascular reactivity.

A. Compared with MG-H1 staining in mesenteric arteries of wild type rats incubated without (panel a1) and with 1.0 mM MGO (panel a2), incubations for 1 hour with $1.0 \mathrm{mM} \mathrm{MGO}$ did not lead to intracellular MG-H1 formation in GLO-I transgenic rats incubated without (panel a3), and with $1.0 \mathrm{mM} \mathrm{MGO}$ (panel a4) (colour figures on page 158). B. In mesenteric arteries of GLO-I transgenic rats, ACh-induced NO-mediated endothelium-dependent relaxation is not impaired during incubation with $1.0 \mathrm{mM} \mathrm{MGO}$ (black circles) for 1 hour compared with control incubations (white squares) $(n=4)$.

\section{Methylglyoxal incubation leads to increased peroxynitrite via the formation of reactive oxygen species}

To investigate a possible role of ROS in the impairment of ACh-induced vasorelaxation by MGO, we stained rat mesenteric arteries for nitrotyrosine after exposure to the endothelial nitric oxide synthase (eNOS) stimulus ACh (figure 3.5A). Nitrosylation of tyrosine residues is a well-known marker for peroxynitrite formation, which is an oxidant product of NO. In parallel to the MG-H1 accumulation, we observed that MGO resulted in an increase in nitrotyrosine staining in the same dose-dependent manner (figure 3.5B). This staining was not limited to specific regions of the vessel wall. MGO induced peroxynitrite formation could be inhibited by treatment of the arteries with the NOS blocker L-NAME (data not shown). 
To confirm that MGO contributes to ROS production in the endothelium, we used a fluorescent probe in endothelial cells to monitor ROS production. In $\mathrm{CM}-\mathrm{H}_{2}$ DCFDA-loaded endothelial cells, MGO treatment resulted in a concentration-dependent increase in oxidative stress formation (figure 3.5C). Direct incubation of the DCF probe with MGO did not lead to any increase in fluorescent signal, thereby excluding a non-specific MGO effect (data not shown). To exclude possible cytotoxic effects of MGO on the endothelial cells, we performed a cell viability assay. The MTT assay showed no significant levels of cytotoxicity after the 1 hour treatment with $0.1,0.33$, and $1.0 \mathrm{mM} \mathrm{MGO}(100 \pm 4 ; 98 \pm 3$; and $97 \pm 5$ percent viability compared to control cells, respectively). In addition, also eNOS staining of the mesenteric arteries showed that the endothelial cell layer remained intact after an exposure to $1.0 \mathrm{mM} \mathrm{MGO}$. Furthermore there were no signs of apoptosis as indicated by the absence in staining of the apoptotic marker cleaved caspase-3 (data not shown).

A
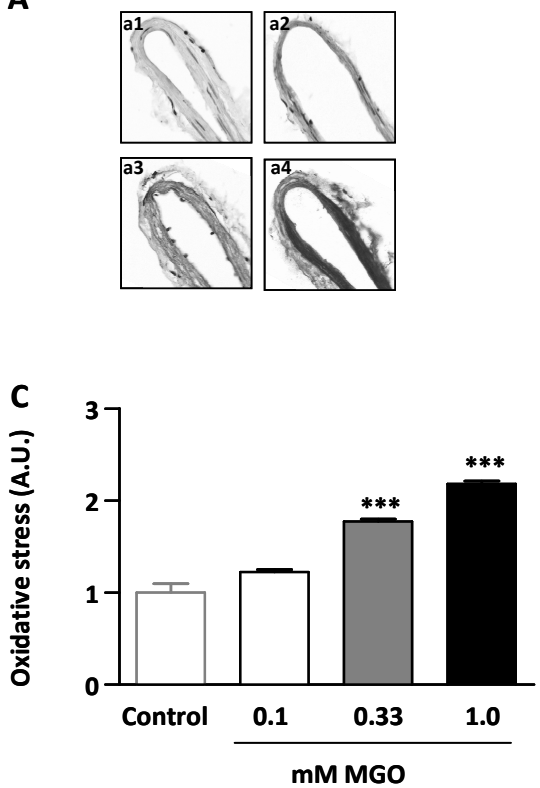

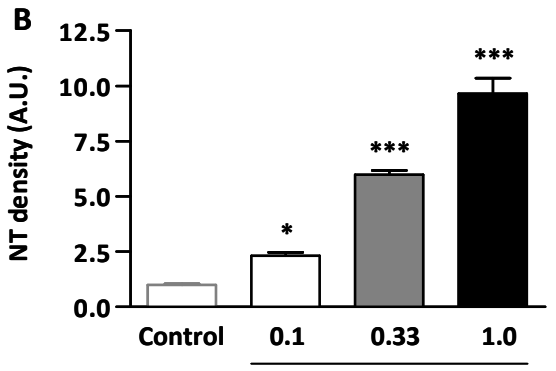

mM MGO

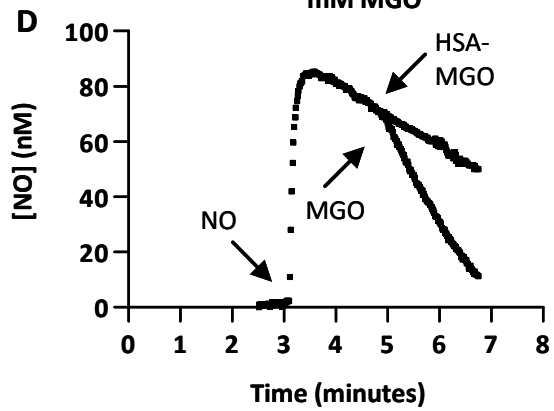

Figure 3.5. Methylglyoxal induced oxidative stress formation in isolated mesenteric arteries and cultured endothelial cells and direct quenching of nitric oxide.

A. Representive staining of nitrotyrosine (NT) in mesenteric resistance arteries not incubated (panel a1) or incubated for 1 hour with $0.1 \mathrm{mM} \mathrm{MGO}$ (panel a2), $0.33 \mathrm{mM} \mathrm{MGO} \mathrm{(panel} \mathrm{a3)} \mathrm{or} 1.0 \mathrm{mM} \mathrm{MGO} \mathrm{(panel} \mathrm{a4)} \mathrm{(colour}$ figures on page 158). B. Quantification of NT staining in MGO-incubated arteries $(n=4)$. C. Oxidative stress

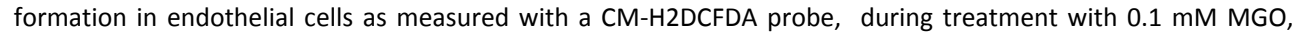
$0.33 \mathrm{mM} \mathrm{MGO}$, and $1.0 \mathrm{mM} \mathrm{MGO}(\mathrm{n}=3)$ for 1 hour. D. Direct effect of methylglyoxal and methylglyoxal-modified human albumin on NO as measured with an Iso-NO meter in deoxygenated buffer. ${ }^{*} \mathrm{p}<0.05$ vs. control group. $* * * p<0.001$ vs. control group. 
We also investigated whether free MGO or MGO-derived AGEs have a direct effect on NO. Free MGO, but not highly modified MGO-albumin, leads to increased breakdown of NO (figure 3.5D). Under the conditions used, the reaction between MGO and NO could be fitted according to a (pseudo) first-order reaction ( $\log k=1.05 \pm 0.13 \mathrm{M}^{-1} \mathrm{~s}^{-1}$ ). The results show that MGO reduced the half-life of $\mathrm{NO}$ in buffer from $4.3 \pm 0.1$ minutes to approximately $0.8 \pm 0.2$ minutes. In comparison, under the same experimental conditions the rate constant found for the potent NO quenching molecule haemoglobin was $1.0 \times 10^{4}$ $\mathrm{M}^{-1} \mathrm{~s}^{-1}(31)$.

\section{Antioxidant treatment prevents impaired vasorelaxation by methylglyoxal}

The results so far suggest a reduction of NO bioavailability by MGO via the induction of ROS formation. In line with this, pre-incubation of the mesenteric arteries with the antioxidants EUK-134, Mn(III)TMP and N-acetyl-cysteine prevented the MGO-impaired AChinduced vasorelaxation (figure 3.6A). In addition also preincubation with the $\mathrm{NAD}(\mathrm{P}) \mathrm{H}$ oxidase inhibitor apocynin prevented the decreased vascular relaxation (see figure 3.7 in the electronic supplementary material, page 64). Vessels incubated with antioxidants showed less nitrotyrosine staining upon incubation with $1.0 \mathrm{mM} \mathrm{MGO}$ (figure 3.6B and C), but MG-H1 levels were not altered (figure 3.6D). In accordance, in cultured endothelial cells the antioxidants also led to reduction of MGO-induced oxidative stress as measured with the $\mathrm{CM}-\mathrm{H}_{2}$ DCFDA probe (figure $3.6 \mathrm{E}$ ).

\section{Discussion}

In this study we have shown that hyperglycaemia-induced impaired endotheliumdependent NO-mediated vasorelaxation of mesenteric arteries is mediated by the major AGE precursor MGO. The effect of MGO occurs intracellularly and is most likely caused by a decreased bioavailability of NO by an MGO-induced increase of oxidative stress. These data provide a mechanistic link between hyperglycaemia, the formation of the AGE precursor MGO and vascular complications.

Endothelial dysfunction is characterized by an impaired vascular response to endotheliumderived NO and is associated with the development of micro- and macro-vascular complications in diabetes (32). To gain insight into the mechanism by which hyperglycaemia alters endothelial function, we used ACh, which stimulates the release of NO from endothelial cells, leading to relaxation of the underlying smooth muscle cells. Experiments were performed in the presence of indomethacin and elevated extracellular $\mathrm{K}^{+}$concentration, to rule out involvements of endothelium-derived prostaglandins and hyperpolarizing factors, and to thereby focus on NO-mediated relaxation. 


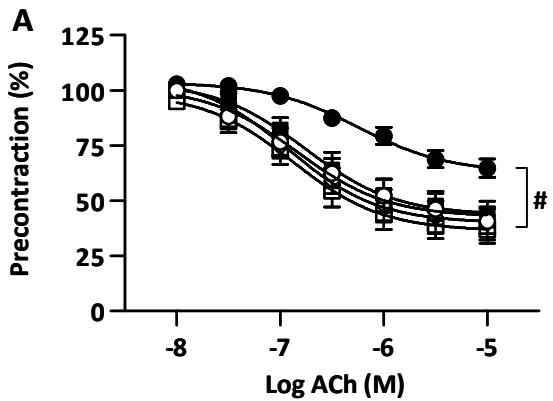

B
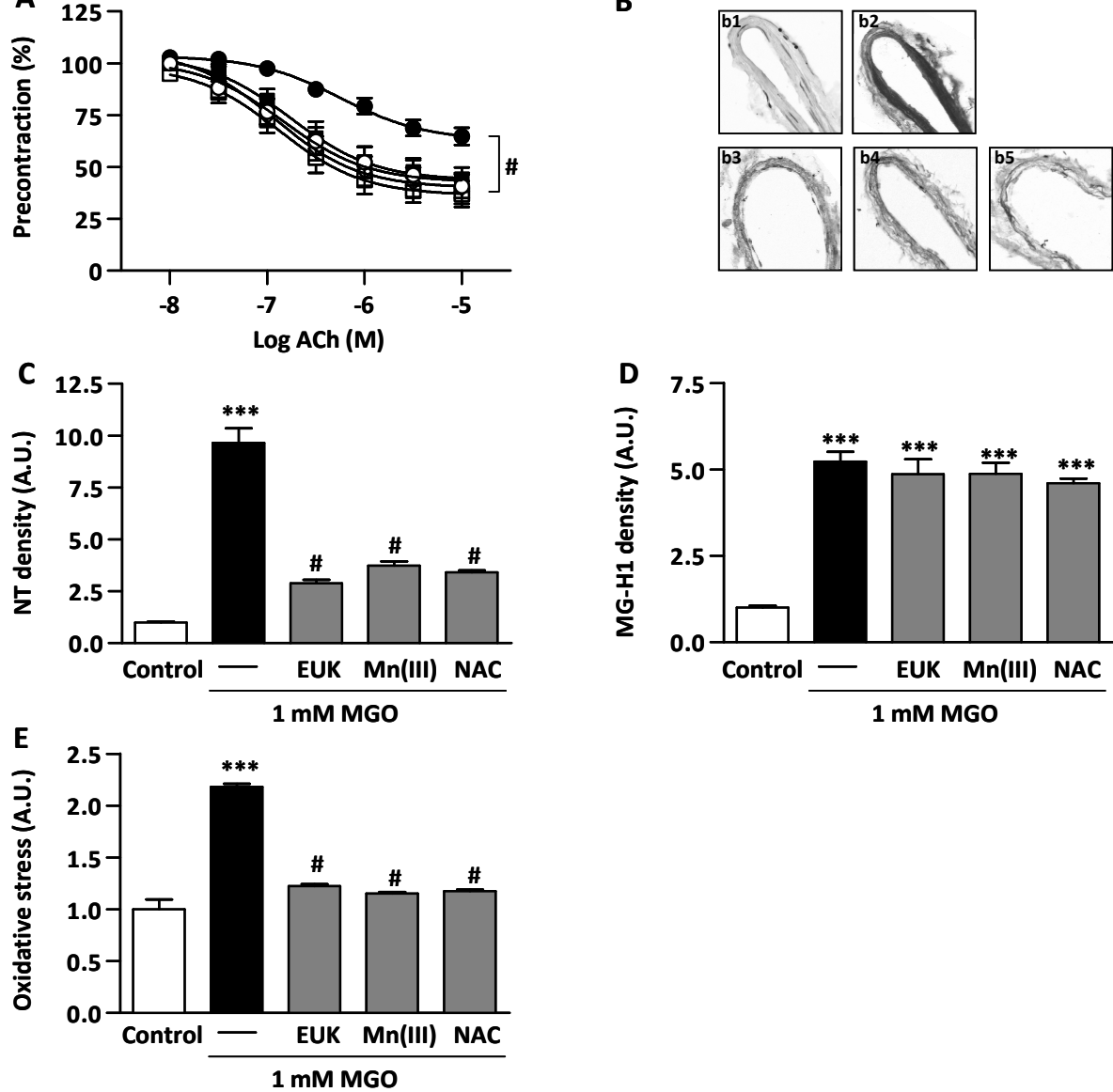

Figure 3.6. Effect of anti-oxidants on the effect of methylglyoxal on vascular reactivity and oxidative stress formation.

A. ACh-induced NO-mediated endothelium-dependent relaxation during incubation without (white squares) or with $1.0 \mathrm{mM} \mathrm{MGO}$ (black circles) for 1 hour with or without pre-incubation with the anti-oxidants $1.0 \mathrm{mM}$ NAC (white diamonds), $1 \mu \mathrm{M}$ EUK-134 (black squares), or $1 \mu \mathrm{M}$ Mn(III)TMP (white circles) for 20 minutes. Treatment of mesenteric arteries with $1.0 \mathrm{mM} \mathrm{MGO}$ in the absence of anti-oxidant results in a significant shift in the doseresponse curve $(\# p<0.001)$ compared with the control group. All anti-oxidant pretreatments prevented MGOinduced impaired reactivity $(n=5)$. B. Nitrotyrosine staining of arteries incubated without (panel b1) or with 1.0 mM MGO (panel b2) and after a pre-incubation with the same concentrations of NAC (panel b3), EUK-134 (panel b4) or Mn(III)TMP (panel b5) (colour figures on page 158). C. Quantification of NT staining in mesenteric arteries after treatment with $1.0 \mathrm{mM} \mathrm{MGO}$ without or with the same concentrations of NAC, EUK-134, or Mn(III)TMP preincubation $(n=4)$. D. Quantification of MG-H1 staining in mesenteric arteries after treatment with 1.0 mM MGO without or with the same concentrations of NAC, EUK-134, or Mn(III)TMP pre-incubation. E. Oxidative stress formation measured with a CM- $\mathrm{H}_{2}$ DCFDA probe in ECRF24 cells after treatment with $1.0 \mathrm{mM}$ MGO without or with $100 \mu \mathrm{M}$ NAC, $10 \mathrm{nM}$ EUK-134 and $10 \mathrm{nM}$ Mn(III)TMP pre-incubation $(\mathrm{n}=3)$. ${ }^{* * *} \mathrm{p}<0.001$ vs. control group. \# $p<0.001$ vs. MGO group. 
We found that treatment of arteries with high concentrations of glucose significantly decreased ACh-induced NO-mediated vasorelaxation (Figure 3.1), which is in agreement with a previous study performed in mesenteric arteries (33). We also found that the effect of high glucose was prevented in arteries with a GLO-I overexpression and moreover that the impaired relaxation in diabetic rats could be improved by GLO-I overexpression. In addition incubation with exogenous MGO resulted in a dose dependent impairment of the ACh-induced vasorelaxation (Figure 3.3).

These data from both ex vivo and in vivo experiments, provide strong evidence that the impaired endothelium-dependent NO-mediated vasorelaxation in diabetes is mediated by the major AGE precursor MGO.

Since the arterial contractile responses to $\mathrm{K}^{+}$and the relaxing responses to the NO-donor SNP were not modified by MGO, our data indicate that a short exposure to MGO leads to an endothelium-dependent effect on vasorelaxation without a direct effect of MGO on arterial smooth muscle function. In accordance with our observations, Mukohda et al. showed that there were no effects of MGO on high potassium-induced contraction (34). Although not tested in our study, it is possible that chronic exposure to exogenous MGO may also lead to impaired endothelium-independent vasorelaxation. In fact, this has been demonstrated in rats with a prolonged exposure to MGO (35).

In contrast to the effect of exogenous MGO, we could not find any effects of extracellularly added MGO-modified albumin on vascular reactivity (Figure 3.2). In accordance with this result, we have previously demonstrated that there is also no binding and biological effect of MGO-modified albumin on endothelial cells (36). In contrast to MGO-albumin, Xu et al. showed that other extracellularly formed AGEs can impair endothelium-dependent vasorelaxation (10). It should be emphasized, however, that these data were obtained by AGE-albumin which was prepared in a degree of modification which is much higher than ever seen in diabetes and the effect seems to be mediated by the AGE-R1 receptor. A recent paper by Gao et al. described the importance of S100b induced RAGE activation in endothelial dysfunction and impaired vasoreactivity (37). We confirmed the finding that the RAGE ligand S100b reduces ACh-induced NO-mediated vasorelaxation (Figure $3.2 \mathrm{C}$ ). Combined, these data provide strong evidence that extracellular MGO-derived AGEs are not involved in diabetic arterial dysfunction and that other ligands for RAGE, such as CML and S100b, possibly impair endothelium-dependent vasorelaxation.

The important finding of our study that the effect of hyperglycaemia and MGO on NOmediated vasorelaxation in rat mesenteric arteries is completely abolished in rat mesenteric arteries with an overexpression of GLO-I, indicates that the effect of hyperglycaemia and exogenous MGO is due to intracellular effects of MGO in endothelial cells. We recently found that endothelial cells incubated with high glucose concentrations 
display an increase in MGO (17) and we now demonstrate that MGO leads to an intracellular accumulation of the major MGO-induced AGE, MG-H1. This is in agreement with a study showing that MGO, which was given intraperitoneally to rats for 7 weeks, induces an increase in vascular AGE content (35). In our study, MG-H1 appeared to be localized in the cytoplasm and in the nucleus of endothelial cells. The latter is in accordance with data of two recent papers about MGO modification of nuclear proteins $(38 ; 39)$. Furthermore Schlotterer et al. recently described that hyperglycaemia leads to MGO modified mitochondrial proteins and reduced lifespan in C. elegans. This oxidative stress dependent process could be inhibited by GLO-I overexpression, thereby linking MGO to the formation of ROS (40). Also other research shows that chronic hyperglycaemia is associated with increased intracellular levels of MGO $(17 ; 41)$, oxidative stress $(42 ; 43)$ and even an impairment of NO synthesis pathways (44). Our results are in line with these studies by showing an oxidative-stress-dependent effect on NO bioavailability by the AGE precursor MGO. This impairment could be prevented by the superoxide dismutase mimetics EUK-134 and Mn(III)TMP and by the antioxidant N-acetyl cysteine (Figure 6). Furthermore the impaired relaxation could also be prevented by the use of apocynin, but due the lack of specificity of this $\mathrm{NAD}(\mathrm{P}) \mathrm{H}$ inhibitor (45), the exact mechanism by which MGO induces the formation of reactive oxygen species is still not completely clear. In addition, although not addressed in this study, the modification of mitochondrial membrane proteins (26), antioxidant enzymes (46) and GAPDH (47) by MGO can increase oxidative stress and thereby impair endothelium-dependent vasorelaxation.

Because AGEs are known to inactivate NO by a direct chemical reaction, the impaired endothelium-dependent vasodilatation in diabetes may be mediated by NO quenching and NO depletion by MGO-derived AGE accumulation (48). Indeed, we observed quenching of NO by MGO, but not by MGO-derived AGEs, and MGO reduced the half-life of NO in buffer from 4 minutes to approximately 1 minute. However, in vivo the very fast reaction of NO with numerous bio-molecules, such as haemoglobin, is one of the reasons why the half-life of NO in biological systems is very short, i.e. less than a second (49), and this half life will not be shortened by MGO. Therefore, the physiological relevance of quenching of $\mathrm{NO}$ by $\mathrm{MGO}$, for example in the MGO-induced impaired endotheliumdependent vasodilatation, is very limited. MGO and MGO-derived AGEs may also decrease NO availability by decreasing NO synthase activity, but in our previous study we already excluded that free MGO and the MGO-derived AGEs arginine-pyrimidine and MG-H1, which have some structural homology with the NOS inhibitor asymmetric dimethylarginine, directly inhibit eNOS activity (50). Together, these findings indicate that the effects of MGO on vasoreactivity are thus caused by MGO-induced oxidative stress and the quenching of NO by oxidative stress, rather than direct interaction of MGO- 
adducts with NO or eNOS activity. Whether MGO or MGO-induced AGEs are involved in impaired eNOS activation by oxidative-stress-related interference with the phosphorylation of eNOS needs further investigated.

For the ex vivo and in vitro experiments we used MGO in a range of $10-1000 \mu \mathrm{M}$ and these concentrations of MGO caused effects in a concentration-dependent manner. The in vivo concentration of MGO is under debate; plasma MGO levels are estimated to be around $0.5 \mu \mathrm{M}$ in healthy individuals and can increase twofold in diabetes (51), while others demonstrated that plasma MGO concentration in poorly controlled human diabetic patients is about $400 \mu \mathrm{M}$ (52). Notwithstanding, cells produce large amounts of MGO (53) and therefore intracellular levels are probably much higher than plasma levels (54). Although the maximum concentration of MGO used in the present study appears to be higher than levels observed in vivo, this concentration was also used to increase the intracellular concentrations and to mimic the life time exposure to elevated levels of MGO such as in diabetes and in hypertension. Under our experimental conditions, the exposure to elevated MGO levels did not lead to cytotoxic effects as indicated by the viability assay, eNOS and cleaved Caspase-3 staining.

In summary, MGO decreased NO-mediated vasorelaxation due to an increase in oxidative stress. Higher plasma levels of MGO have been demonstrated under both postprandial (35) and basal hyperglycaemic conditions $(16,17,42)$, therefore we propose here that MGO is crucial in impaired endothelium-dependent vasorelaxation under these conditions and, in the long term, for the development of vascular complications. We conclude that hyperglycaemia-induced impairment of endothelium-dependent vasorelaxation is mediated by intracellular MGO levels in an oxidative stress-dependent pathway. These data provide a new mechanistic link between hyperglycaemia, the formation of MGOderived AGEs and vascular complications.

\section{Acknowledgements}

This study was partially supported by the Dutch Diabetes Foundation (Grant number 2005.11.013). 


\section{Electronic supplementary material}

\section{Cont'd Methods}

\section{Isometric tension measurements}

Healthy adult male Wistar rats, or adult Wistar rats with a human GLO-I overexpression (GLO-I TG) (300 - 450 gram) were sacrificed by $\mathrm{CO}_{2}$ inhalation. The viscera were exposed in order to remove the superior mesenteric vascular arcade which was then pinned onto a dissecting dish containing cold Krebs-Ringer bicarbonate solution (KRB): $118 \mathrm{mM} \mathrm{NaCl} ; 4.7$ $\mathrm{mM} \mathrm{KCl} ; 1.2 \mathrm{mM} \mathrm{KH}_{2} \mathrm{PO}_{4} ; 25 \mathrm{mM} \mathrm{NaHCO}_{3} ; 1.1 \mathrm{mM} \mathrm{MgSO}_{4} ; 2.5 \mathrm{mM} \mathrm{CaCl}_{2}$ and $5.0 \mathrm{mM}$ glucose. A second order branch of the superior mesenteric artery was cleaned of fat and connective tissue, and a segment of approximately $8.0 \mathrm{~mm}$ in length was removed and divided in 4 equal segments. These segments were then mounted in a myograph organ bath (model 610M Danish Myotechnology by J.P. Trading, Denmark) with two steel $40-\mu \mathrm{m}$ wires inserted through the lumen of the segments. After mounting the tissues, the organ bath solution was changed to fresh Krebs maintained at $37^{\circ} \mathrm{C}$ and gassed continuously with $95 \% \mathrm{O}_{2}$ and $5 \% \mathrm{CO}_{2}$ (pH 7.4). Prior to the normalization procedure, the arteries were rested for 15 minutes without tension. The internal diameter of each vessel was normalized as described by Halpern and Mulvany (55). This was performed by stretching the vessel to a diameter that yielded a wall tension equivalent to $90 \%$ of that given by a transmural pressure of $100 \mathrm{mmHg}$ (this was $326 \pm 37 \mu \mathrm{m}$ for wild type rats and $336 \pm 25 \mu \mathrm{m}$ for GLO-I transgenic rats). The isometric tension generated by the vessels was recorded using Powerlab 4/25 (ADInstruments) connected to the Myo-Interface. Endothelial viability was assessed by application of $10 \mu \mathrm{M}$ acetylcholine (ACh) during contraction with $10 \mu \mathrm{M}$ phenylephrine. Viable endothelium resulted in $94 \pm 6 \%$ relaxation. NO-mediated effects were investigated using $A C h,(0.01-10 \mu \mathrm{M})$ and sodium nitroprusside (SNP, $0.01-$ $10 \mu \mathrm{M})$ during contraction with $65 \mathrm{mM} \mathrm{K}^{+}$which blocks Endothelium Derived Hyperpolarizing Factors (EDHFs). NO-mediated vasorelaxation was measured after incubation with or without MGO after pre-treatment with or without the antioxidant $\mathrm{N}$ acetyl cysteine (NAC) (Sigma Aldrich) or the superoxide dismutase mimetics EUK-134 and MnIIITMP (Invitrogen, San Diego, USA). During some experiments arteries were exposed to additional high glucose (25 and $35 \mathrm{mM}$, Sigma Aldrich) or mannitol ( $35 \mathrm{mM}$, Sigma Aldrich). During the experiments, $10 \mu \mathrm{M}$ indomethacin was used to block the synthesis of prostaglandins. Iso-osmotic buffer containing $65 \mathrm{mM} \mathrm{K}^{+}$was prepared by mixing appropriate volumes of $\mathrm{KRB}$ with a $\mathrm{KRB}$ in which all $\mathrm{NaCl}$ was replaced by $\mathrm{KCl}$.

\section{NO measurement}

Deoxygenated 50mM phosphate buffer ( $\mathrm{pH} 7.4$ ) was prepared under a N2 atmosphere. Deoxygenated water was spiked with $\bullet N O$ gas for about 1 minute, and $2 \mu$ of the $\bullet$ NO 
spiked water was added to $20 \mathrm{~mL} 50 \mathrm{mM}$ phosphate buffer ( $\mathrm{pH} 7.4)$ in a thermostatted test tube $\left(37^{\circ} \mathrm{C}\right)$. During measurements the test tube was kept under a N2 atmosphere. The $\bullet N O$ concentration was monitored with an Iso-NO meter (world Precision Instruments, Sarasota FL, USA), which was coupled to a MacLab ${ }^{\text {TM }}$ interface (ML020 MacLab/8, ADInstruments, London, UK) and recorded with 'Chart' software (Kipp, Delft, The Netherlands). The decrease in •NO concentration was followed in time in the presence or absence of the test compound in solution. The stock solution of the test compound was incubated at $37{ }^{\circ} \mathrm{C}$ for $10 \mathrm{~min}$ before use. During this procedure, the solution in the test vessel was mixed using a magnetic stirrer. The natural logarithm of the -NO concentration was plotted versus time. For the determination of the rate constant, the $\bullet N O$ concentration was expressed in arbitrary units, since the reaction showed (pseudo) first-order reaction kinetics.

\section{Results ESM}

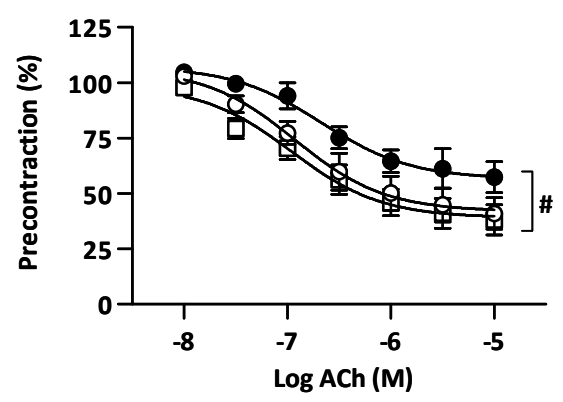

Figure 3.7. Inhibition of $\mathrm{NAD}(\mathrm{P}) \mathrm{H}$ oxidase normalises methylglyoxal-induced impaired NO-mediated relaxation. ACh-induced NO-mediated endothelium-dependent relaxation during incubation without (white squares) or with $1.0 \mathrm{mM} \mathrm{MGO}$ (black circles) and with $1.0 \mathrm{mM} \mathrm{MGO}$ after 20 minutes pre-treatment with $100 \mu \mathrm{M}$ apocynin (white circles). Preincubation with apocynin prevents the impaired NO-mediated relaxation induced by $1.0 \mathrm{mM}$ MGO for 1 hour $(\# p<0.05)(n=5)$.

\section{Reference ESM}

[1] Halpern W, Mulvany MJ (1977) Tension responses to small length changes of vascular smooth muscle cells [proceedings]. J Physiol 265: 21P-23P 


\section{References}

1. Steinberg HO, Chaker H, Leaming R, Johnson A, Brechtel G, Baron AD: Obesity/insulin resistance is associated with endothelial dysfunction. Implications for the syndrome of insulin resistance. J Clin Invest 97:2601-2610, 1996

2. Makimattila S, Virkamaki A, Groop PH, Cockcroft J, Utriainen T, Fagerudd J, Yki-Jarvinen H: Chronic hyperglycaemia impairs endothelial function and insulin sensitivity via different mechanisms in insulindependent diabetes mellitus. Circulation 94:1276-1282, 1996

3. Diederich D, Skopec J, Diederich A, Dai FX: Endothelial dysfunction in mesenteric resistance arteries of diabetic rats: role of free radicals. Am J Physiol 266:H1153-1161, 1994

4. Pflueger AC, Osswald H, Knox FG: Adenosine-induced renal vasoconstriction in diabetes mellitus rats: role of nitric oxide. Am J Physiol 276:F340-346, 1999

5. Brownlee M: Biochemistry and molecular cell biology of diabetic complications. Nature 414:813-820, 2001

6. Ono Y, Aoki S, Ohnishi K, Yasuda T, Kawano K, Tsukada Y: Increased serum levels of advanced glycation end products in NIDDM patients with diabetic complications. Diabetes Care 21:1027, 1998

7. Hammes HP, Brownlee M, Lin J, Schleicher E, Bretzel RG: Diabetic retinopathy risk correlates with intracellular concentrations of the glycoxidation product Nepsilon-(carboxymethyl) lysine independently of glycohaemoglobin concentrations. Diabetologia 42:603-607, 1999

8. Kilhovd BK, Berg TJ, Birkeland KI, Thorsby P, Hanssen KF: Serum levels of advanced glycation end products are increased in patients with type 2 diabetes and coronary heart disease. Diabetes Care 22:1543-1548, 1999

9. Tan KC, Chow WS, Ai VH, Metz C, Bucala R, Lam KS: Advanced glycation end products and endothelial dysfunction in type 2 diabetes. Diabetes Care 25:1055-1059, 2002

10. Xu B, Chibber R, Ruggiero D, Kohner E, Ritter J, Ferro A: Impairment of vascular endothelial nitric oxide synthase activity by advanced glycation end products. Faseb J 17:1289-1291, 2003

11. Beisswenger PJ, Howell SK, Nelson RG, Mauer M, Szwergold BS: Alpha-oxoaldehyde metabolism and diabetic complications. Biochem Soc Trans 31:1358-1363, 2003

12. Shinohara M, Thornalley PJ, Giardino I, Beisswenger P, Thorpe SR, Onorato J, Brownlee M: Overexpression of glyoxalase-I in bovine endothelial cells inhibits intracellular advanced glycation endproduct formation and prevents hyperglycaemia-induced increases in macromolecular endocytosis. J Clin Invest 101:1142-1147, 1998

13. Thornalley PJ: Glyoxalase l--structure, function and a critical role in the enzymatic defence against glycation. Biochem Soc Trans 31:1343-1348, 2003

14. Oya T, Hattori N, Mizuno Y, Miyata S, Maeda S, Osawa T, Uchida K: Methylglyoxal modification of protein. Chemical and immunochemical characterization of methylglyoxal-arginine adducts. J Biol Chem 274:1849218502, 1999

15. Ahmed N, Thornalley PJ: Chromatographic assay of glycation adducts in human serum albumin glycated in vitro by derivatization with 6-aminoquinolyl-N-hydroxysuccinimidyl-carbamate and intrinsic fluorescence. Biochem J 364:15-24, 2002

16. McLellan AC, Thornalley PJ, Benn J, Sonksen PH: Glyoxalase system in clinical diabetes mellitus and correlation with diabetic complications. Clin Sci (Lond) 87:21-29, 1994

17. Schalkwijk CG, van Bezu J, van der Schors RC, Uchida K, Stehouwer CD, van Hinsbergh VW: Heat-shock protein 27 is a major methylglyoxal-modified protein in endothelial cells. FEBS Lett 580:1565-1570, 2006

18. Mostafa AA, Randell EW, Vasdev SC, Gill VD, Han Y, Gadag V, Raouf AA, El Said H: Plasma protein advanced glycation end products, carboxymethyl cysteine, and carboxyethyl cysteine, are elevated and related to nephropathy in patients with diabetes. Mol Cell Biochem 302:35-42, 2007

19. Fosmark DS, Torjesen PA, Kilhovd BK, Berg TJ, Sandvik L, Hanssen KF, Agardh CD, Agardh E: Increased serum levels of the specific advanced glycation end product methylglyoxal-derived Nס-(5-hydro-5-methyl-4-imidazolon2-yl)-L-ornithine are associated with retinopathy in patients with type 2 diabetes mellitus. Metabolism 55:232236, 2006

20. Wang $X$, Chang T, Jiang B, Desai K, Wu L: Attenuation of hypertension development by aminoguanidine in spontaneously hypertensive rats: role of methylglyoxal. Am J Hypertens 20:629-636, 2007

21. Wang X, Desai K, Chang T, Wu L: Vascular methylglyoxal metabolism and the development of hypertension. J Hypertens 23:1565-1573, 2005

22. Wang X, Desai K, Clausen JT, Wu L: Increased methylglyoxal and advanced glycation end products in kidney from spontaneously hypertensive rats. Kidney Int 66:2315-2321, 2004 
23. Wang X, Jia X, Chang T, Desai K, Wu L: Attenuation of hypertension development by scavenging methylglyoxal in fructose-treated rats. J Hypertens 26:765-772, 2008

24. Wu L, Juurlink BH: Increased methylglyoxal and oxidative stress in hypertensive rat vascular smooth muscle cells. Hypertension 39:809-814, 2002

25. Nemet I, Varga-Defterdarovic L, Turk Z: Methylglyoxal in food and living organisms. Mol Nutr Food Res 50:1105-1117, 2006

26. Rosca MG, Mustata TG, Kinter MT, Ozdemir AM, Kern TS, Szweda LI, Brownlee M, Monnier VM, Weiss MF: Glycation of mitochondrial proteins from diabetic rat kidney is associated with excess superoxide formation. Am J Physiol Renal Physiol 289:F420-430, 2005

27. Inagi R, Miyata T, Ueda Y, Yoshino A, Nangaku M, van Ypersele de Strihou C, Kurokawa K: Efficient in vitro lowering of carbonyl stress by the glyoxalase system in conventional glucose peritoneal dialysis fluid. Kidney Int 62:679-687, 2002

28. Scheijen JL, van de Waarenburg MP, Stehouwer CD, Schalkwijk CG: Measurement of pentosidine in human plasma protein by a single-column high-performance liquid chromatography method with fluorescence detection. J Chromatogr B Analyt Technol Biomed Life Sci 877:610-614, 2009

29. Fontijn R, Hop C, Brinkman HJ, Slater R, Westerveld A, van Mourik JA, Pannekoek H: Maintenance of vascular endothelial cell-specific properties after immortalization with an amphotrophic replication-deficient retrovirus containing human papilloma virus 16 E6/E7 DNA. Exp Cell Res 216:199-207, 1995

30. McLellan AC, Phillips SA, Thornalley PJ: The assay of S-D-lactoylglutathione in biological systems. Anal Biochem 211:37-43, 1993

31. Vriesman MF, Haenen GR, Westerveld GJ, Paquay JB, Voss HP, Bast A: A method for measuring nitric oxide radical scavenging activity. Scavenging properties of sulfur-containing compounds. Pharm World Sci 19:283-286, 1997

32. Cooke JP, Dzau VJ: Nitric oxide synthase: role in the genesis of vascular disease. Annu Rev Med 48:489-509, 1997

33. Taylor PD, Poston L: The effect of hyperglycaemia on function of rat isolated mesenteric resistance artery. $\mathrm{Br}$ J Pharmacol 113:801-808, 1994

34. Mukohda M, Yamawaki H, Nomura H, Okada M, Hara Y: Methylglyoxal inhibits smooth muscle contraction in isolated blood vessels. J Pharmacol Sci 109:305-310, 2009

35. Berlanga J, Cibrian D, Guillen I, Freyre F, Alba JS, Lopez-Saura P, Merino N, Aldama A, Quintela AM, Triana ME, Montequin JF, Ajamieh H, Urquiza D, Ahmed N, Thornalley PJ: Methylglyoxal administration induces diabetes-like microvascular changes and perturbs the healing process of cutaneous wounds. Clin Sci (Lond) 109:83-95, 2005

36. Lieuw-a-Fa ML, Schalkwijk CG, Engelse M, van Hinsbergh VW: Interaction of Nepsilon(carboxymethyl)lysineand methylglyoxal-modified albumin with endothelial cells and macrophages. Splice variants of RAGE may limit the responsiveness of human endothelial cells to AGEs. Thromb Haemost 95:320-328, 2006

37. Gao X, Zhang H, Schmidt AM, Zhang C: AGE/RAGE produces endothelial dysfunction in coronary arterioles in type 2 diabetic mice. Am J Physiol Heart Circ Physiol 295:H491-498, 2008

38. Ceradini DJ, Yao D, Grogan RH, Callaghan MJ, Edelstein D, Brownlee M, Gurtner GC: Decreasing intracellular superoxide corrects defective ischemia-induced new vessel formation in diabetic mice. J Biol Chem 283:1093010938, 2008

39. Yao D, Taguchi T, Matsumura T, Pestell R, Edelstein D, Giardino I, Suske G, Rabbani N, Thornalley PJ, Sarthy VP, Hammes HP, Brownlee M: High glucose increases angiopoietin-2 transcription in microvascular endothelial cells through methylglyoxal modification of mSin3A. J Biol Chem 282:31038-31045, 2007

40. Schlotterer A, Kukudov G, Bozorgmehr F, Hutter H, Du X, Oikonomou D, Ibrahim Y, Pfisterer F, Rabbani N, Thornalley P, Sayed A, Fleming T, Humpert P, Schwenger V, Zeier M, Hamann A, Stern D, Brownlee M, Bierhaus A, Nawroth P, Morcos M: C. elegans as model for the study of high glucose mediated lifespan reduction. Diabetes, 2009

41. Thornalley PJ, Battah S, Ahmed N, Karachalias N, Agalou S, Babaei-Jadidi R, Dawnay A: Quantitative screening of advanced glycation endproducts in cellular and extracellular proteins by tandem mass spectrometry. Biochem J 375:581-592, 2003

42. Forbes JM, Coughlan MT, Cooper ME: Oxidative stress as a major culprit in kidney disease in diabetes. Diabetes 57:1446-1454, 2008

43. Vincent AM, Brownlee M, Russell JW: Oxidative stress and programmed cell death in diabetic neuropathy. Ann N Y Acad Sci 959:368-383, 2002 
44. Su J, Lucchesi PA, Gonzalez-Villalobos RA, Palen DI, Rezk BM, Suzuki Y, Boulares HA, Matrougui K: Role of Advanced Glycation End Products With Oxidative Stress in Resistance Artery Dysfunction in Type 2 Diabetic Mice. Arterioscler Thromb Vasc Biol, 2008

45. Heumuller S, Wind S, Barbosa-Sicard E, Schmidt HH, Busse R, Schroder K, Brandes RP: Apocynin is not an inhibitor of vascular NADPH oxidases but an antioxidant. Hypertension 51:211-217, 2008

46. Choudhary D, Chandra D, Kale RK: Influence of methylglyoxal on antioxidant enzymes and oxidative damage. Toxicol Lett 93:141-152, 1997

47. Lee HJ, Howell SK, Sanford RJ, Beisswenger PJ: Methylglyoxal can modify GAPDH activity and structure. Ann N Y Acad Sci 1043:135-145, 2005

48. Bucala R, Tracey KJ, Cerami A: Advanced glycosylation products quench nitric oxide and mediate defective endothelium-dependent vasodilatation in experimental diabetes. J Clin Invest 87:432-438, 1991

49. Liu X, Miller MJ, Joshi MS, Sadowska-Krowicka H, Clark DA, Lancaster JR, Jr.: Diffusion-limited reaction of free nitric oxide with erythrocytes. J Biol Chem 273:18709-18713, 1998

50. Brouwers O, Teerlink T, van Bezu J, Barto R, Stehouwer C, Schalkwijk C: Methylglyoxal and methylglyoxalarginine adducts do not directly inhibit endothelial nitric oxide synthase. Ann N Y Acad Sci, 2007

51. Lapolla A, Flamini R, Dalla Vedova A, Senesi A, Reitano R, Fedele D, Basso E, Seraglia R, Traldi P: Glyoxal and methylglyoxal levels in diabetic patients: quantitative determination by a new GC/MS method. Clin Chem Lab Med 41:1166-1173, 2003

52. Lapolla A, Reitano R, Seraglia R, Sartore G, Ragazzi E, Traldi P: Evaluation of advanced glycation end products and carbonyl compounds in patients with different conditions of oxidative stress. Mol Nutr Food Res 49:685-690, 2005

53. Chaplen FW, Fahl WE, Cameron DC: Evidence of high levels of methylglyoxal in cultured Chinese hamster ovary cells. Proc Natl Acad Sci U S A 95:5533-5538, 1998

54. Randell EW, Vasdev S, Gill V: Measurement of methylglyoxal in rat tissues by electrospray ionization mass spectrometry and liquid chromatography. J Pharmacol Toxicol Methods 51:153-157, 2005

55. Halpern W, Mulvany MJ: Tension responses to small length changes of vascular smooth muscle cells [proceedings]. J Physiol 265:21P-23P, 1977 
Chapter 3 
Overexpression of glyoxalase-I reduces hyperglycaemiainduced levels of advanced glycation endproducts and oxidative stress in diabetic rats

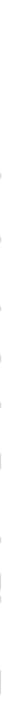

Olaf Brouwers, Petra Niessen, Isabel Ferreira, Toshio Miyata, Peter Scheffer, Tom Teerlink, Patrick Schrauwen, Michael Brownlee, Coen Stehouwer and Casper Schalkwijk J Biol Chem. 2011 Jan; 286(2): 1374-1380. 


\section{Summary}

The reactive advanced glycation endproduct ( $A G E$ ) precursor methylglyoxal (MGO) and MGO-derived AGEs are associated with diabetic vascular complications and also with an increase in oxidative stress. Glyoxalase-I (GLO-I) transgenic rats were used to explore whether overexpression of this MGO detoxifying enzyme reduces levels of AGEs and oxidative stress in a rat model of diabetes.

Rats were made diabetic with streptozotocin and after 12 weeks, plasma and multiple tissues were isolated for analysis of AGEs, carbonyl stress and oxidative stress. GLO-I activity was significantly elevated in multiple tissues of all transgenic rats compared with wild type (WT) littermates. Streptozotocin treatment resulted in a fivefold increase in blood glucose concentrations irrespective of GLO-I overexpression. Levels of MGO, glyoxal (GO), 3-deoxyglucosone, AGEs and oxidative stress markers nitrotyrosine, malondialdehyde and F2-isoprostane were elevated in the diabetic WT rats. In diabetic GLO-I rats, GO and MGO composite scores were significantly decreased by $81 \%$, and plasma AGEs and oxidative stress markers scores by approximately $50 \%$. Hyperglycaemia induced a decrease in protein levels of the mitochondrial oxidative phosphorylation complex in the gastrocnemius muscle, which was accompanied by an increase in the lipid peroxidation product 4-hydroxy-2-nonenal, and this was counteracted by GLO-I overexpression.

This study shows for the first time, in an in vivo model of diabetes, that GLO-I overexpression reduces hyperglycaemia-induced levels of carbonyl stress, AGEs and oxidative stress. The reduction of oxidative stress by GLO-I overexpression directly demonstrates the link between glycation and oxidative stress. 


\section{Introduction}

Prolonged exposure to hyperglycaemia has detrimental effects on various cellular functions and is believed to be the most important factor in the development of vascular complications in diabetes. One of the hypotheses about how hyperglycaemia leads to complications is the formation of advanced glycation endproducts (AGEs) (1). In addition to the formation of AGEs by the classical Maillard reaction $(2 ; 3)$, dicarbonyls such as methylglyoxal (MGO), glyoxal (GO) and 3-deoxyglucocone (3-DG) are also known to form AGEs. MGO is probably the most important precursor in this formation of AGEs (4). This highly reactive oxo-aldehyde is formed mainly by the non-enzymatic and enzymatic fragmentation of the triose phosphates glyceraldehyde-3-phosphate and di-hydroxyacetone-phosphate. MGO reacts primarily with arginine residues to form the major AGEs, $\mathrm{N}_{\delta}$-(5-hydro-5-methyl-4-imidazolon-2-yl)-L-ornithine (MG-H1), arginine-pyrimidine (AP) and tetrahydropyrimidine (THP), and with lysine residues to minor AGEs such as $\mathrm{N}_{\varepsilon}-(1-$ carboxyethyl)lysine (CEL). MGO is efficiently detoxified by the glyoxalase system. In this pathway MGO reacts with reduced glutathione $(\mathrm{GSH})$ to a hemithioacetal adduct, and then to S-d-lactoyl-gluthathione which is catalysed by glyoxalase-I (GLO-I). This product is converted into D-lactate by glyoxalase-II, thereby reforming the consumed GSH. A similar reaction with glyoxal can take place via the intermediate S-glycolylglutathione (5). Overexpression of GLO-I prevents the accumulation of MGO and AGEs in endothelial cells exposed to high glucose concentration in vitro, indicating that GLO-I plays an important role in the suppression of AGE formation under hyperglycaemic condition (6).

Among other adverse effects, MGO induces dysfunction of mitochondrial respiration (7) and increases the production of reactive oxygen species (ROS) (8; 9). Also the decrease of GLO-I activity by an inhibitor results in altered cellular function (10-12). Recently Schlotterer et al. showed that GLO-I overexpression in C. elegans decreased hyperglycaemia-induced accumulation of AGEs and oxidative stress and enhanced lifespan (13). Nevertheless, in higher organisms the in vivo effect of GLO-I overexpression on hyperglycaemia induced AGE formation and ROS production has not yet been studied. Therefore in this study we investigated the phenotype of diabetic transgenic rats with a GLO-I overexpression. We hypothesized that these rats will have less MGO and MGOderived adducts in comparison with their wild-type diabetic littermates, and that this would have beneficial effects on the formation of hyperglycaemia-induced ROS production.

\section{Methods}

\section{Glyoxalase-I transgenic rats}

To generate the human GLO-I transgene construct, the entire coding sequence of human GLO-I cDNA was cloned into the EcoR I site of the pBsCAG-2. 
The GLO-I transgene, isolated by digestion of pBsCAG-2 containing GLO I-cDNA with Kpn I and Sac I, was microinjected into one pronucleus of fertilized Wistar eggs, followed by transfer into the oviducts of pseudopregnant rats. The procedure used was comparable with the generation of glyoxalase-I overexpressing mice (14). Rat genomic DNA extracted from tail tissue was used to detect the transgene by polymerase chain reaction (PCR) using specific primers for GLO-I or pBSCAG-2 vector. Primers for cytomegalovirus enhancer sense (5'-GTC GAC ATT GAT TAT TGA CTA G-3') and antisense (5'-CCA TAA GGT CAT GTA CTG-3') amplified a $350 \mathrm{bp}$ fragment. Primers for the fragment containing human GLO I gene and 3' junction of vector, sense (5'-GTA GTG TGG GTG ACT CCT CCG TTC CTT GGG-3') and antisense (5'-TCG AGG GAT CTT CAT AAG AGA AGA G-3'), amplified a 1200 bp fragment. PCR amplification was carried out with an initial denaturation at $94^{\circ} \mathrm{C}$ for two minutes, followed by 35 cycles of $95^{\circ} \mathrm{C}$ for 30 seconds, $55^{\circ} \mathrm{C}$ or $58^{\circ} \mathrm{C}$ for 30 seconds, and $72^{\circ} \mathrm{C}$ for 30 or 75 seconds.

\section{Induction of diabetes with streptozotocin}

All animal studies were carried out in accordance with the Guide for the Care and Use of Laboratory Animals of the National Institutes of Health. All experiments involving rats were reviewed and approved by the ethics committee for animal care and use of the Maastricht University, the Netherlands.

Wild-type rats (WtD, $n=9)$ and transgenic GLO-I rats (TgD, $n=8)$ were made diabetic by intravenous injection of streptozotocin (STZ, $65 \mathrm{mg} / \mathrm{kg}$ body weight). Weight- and agematched control rats (WtC, $n=9)$ and transgenic $\mathrm{GLO}-\mathrm{I}$ rats $(\mathrm{TgC}, \mathrm{n}=8)$ were not injected. Blood glucose levels were monitored at baseline and after 1 and 12 weeks. Only STZinjected rats with a fasting blood glucose level above $15 \mathrm{mM}$ after 1 and 12 weeks were included in the study as diabetic. Body weight, food and fluid intake, urine production, and urinary creatinine levels were measured at baseline and after 12 weeks. Three months after the induction of diabetes, rats were sacrificed and multiple tissues were processed for analysis. Samples from various organs were fixed in $4 \%$ neutral buffered formaldehyde, embedded in paraffin, and sectioned at $4 \mu \mathrm{m}$ thickness. Blood samples were collected at the time of sacrifice for haematological and biochemical analyses. Tissues (50 $\mathrm{mg}$ ) from wild-type or GLO-I transgenic rats were homogenized in $1 \mathrm{~mL}$ of $0.1 \mathrm{M}$ sodium phosphate buffer, $\mathrm{pH}$ 7.0, containing $0.02 \%$ TritonX-100 and centrifuged at 20,000 xg for 20 minutes at $4^{\circ} \mathrm{C}$. The supernatant was used as a tissue lysate to assess GLO-I protein expression with Western blotting and GLO-I activity.

\section{Preparation of anti-MGO antibodies}

MGO-keyhole limpet hemocyanin (KLH) was prepared by the reaction of MGO (10 mM) with $\mathrm{KLH}$ for 7 days at $37^{\circ} \mathrm{C}$ and was used as antigen for the immunization of mice. MGO- 
$\mathrm{KLH}$ was emulsified in an equal volume of Freund's complete adjuvant and three mice were intradermally injected at multiple sites. The mice were boosted with the same amount of MGO-KLH emulsified in Freund's incomplete adjuvant 21 days later and antisera obtained 14 days after the booster were tested. The booster was repeated twice. Ten days after the final booster, antisera were tested with MGO-albumin and the mouse with the highest titre was used for fusion. We obtained 40 positive clones as tested with MGO-albumin and one of them was further characterized. This monoclonal antibody was from the IgG1 subclass, and has an at least 1000 -fold preference for tetrahydropyrimidine as compared to arginine-pyrimidine or 5-hydro-5-methyl-4-imidazolone.

\section{Glyoxalase-I activity measurement}

GLO-I activity was assayed by spectrophotometry by monitoring the increase in absorbance at $240 \mathrm{~nm}$ due to the formation of S-D-lactoylglutathione for ten minutes at $37^{\circ} \mathrm{C}$ according to the method by McLellan and Thornalley (15).

\section{Preparation of MGO-albumin}

Human serum albumin $(6.8 \mathrm{mg})$ was dialyzed against phosphate buffer $(0.1 \mathrm{M})$ and filtrated through a $0.45 \mu \mathrm{m}$ filter followed by incubation in a phosphate buffer $(0.2 \mathrm{M}), \mathrm{pH}$ 7.8 , with different concentrations of MGO (0.5-10 mM).

\section{MGO-albumin Elisa}

In a competitive Elisa, performed at room temperature, each well was coated with $100 \mu \mathrm{l}$ of $0.1 \mathrm{mg} / \mathrm{ml} \mathrm{MGO-albumin}$, as prepared by the incubation of albumin with $0.5 \mathrm{mM} \mathrm{MGO}$ for 2 days, in PBS for 1 hour at room temperature or overnight at $4^{\circ} \mathrm{C}$. This MGO-albumin preparation was also used as standard in the competitive Elisa. To each well were added $50 \mu \mathrm{l}$ of the antibody (anti-THP (1:2000) or anti MG-H1 (1:100.000)), and $50 \mu \mathrm{l}$ of standard or plasma sample to be tested. After incubation for 1 hour and three washes with PBSTween, the wells were incubated with $100 \mu \mathrm{l}$ peroxidase-labeled goat-anti-mouse (1:5000, Dako, Glostrup, Denmark) for 1 hour. Finally, the wells were washed five times with PBSTween and the substrate was developed with $100 \mu \mathrm{l}$ of tetramethylbenzidine. The reaction was stopped with $100 \mu \mathrm{l} 0.5 \mathrm{M} \mathrm{H}_{2} \mathrm{SO}_{4}$. The extinction at $450 \mathrm{~nm}$ was measured with a multichannel spectrophotometer (SLT Microplate reader, Wilten Bioteknika, EttenLeur, the Netherlands). Plasma levels were expressed as arbitrary units, and one unit was defined as the antibody-reactive material equivalent to $1 \mu \mathrm{g}$ of the MGO-albumin standard. 


\section{Measurement of oxo-aldehydes and AGEs}

Blood samples were collected in $0.8 \mathrm{M}$ perchloric acid (PCA) and concentrations of the dicarbonyls MGO and GO were determined by reversed-phase high-performance liquid chromatography (HPLC) with fluorescence detection after derivatization to their respective dimethoxyquinoxaline derivatives (16). Concentrations of the dicarbonyl 3-DG were measured with HPLC tandem mass spectrometry after derivatization with dinitrophenylhydrazine (17).

In addition, protein-bound CML and CEL were measured in plasma by HPLC tandem mass spectrometry (18). Protein-bound Arginine-pyrimidine was measured by HPLC with fluorescent detection with the same sample preparation as described by Scheijen et al. (19) for pentosidine.

\section{Measurement of D-lactate and markers of oxidative stress}

D-lactate was measured in blood and urine by an enzymatic assay with spectrophotometric quantification (20).

8-Isoprostane (21) and malondialdehyde (MDA) (22) levels were measured in rat urine by HPLC with MS-MS or fluorescence detection, respectively.

\section{Western blot analysis of mitochondrial proteins and oxidative stress markers}

Western blot analyses were performed in muscle homogenates of the gastrocnemius muscle using an antibody cocktail (MS601; MitoSciences, Eugene, OR) against structural components of four of the five oxidative phosphorylation complexes (i.e., to the ND6 subunit of complex I, the $30 \mathrm{kDa}$ Ip subunit of complex II, the $47 \mathrm{kDa}$ core protein 2 of complex III, and the $\alpha$-subunit of the F1F0 ATP synthase of complex V).

In the whole muscle homogenates, we also analyzed uncoupling protein-3 (UCP-3), adenine nucleotide translocase-1 (ANT-1) (MSA02; MitoSciences) and 4-hydroxy-2nonenal (4-HNE) (JAICA; Fukuroi, Japan) protein content by western blotting according to Hoeks et al. (23). The nitric oxide oxidation product, peroxynitrite (polyclonal nitrotyrosine antibody from, Calbiochem, San Diego, USA), was detected in kidney lysates. In brief, upon homogenization in lysis buffer, containing 1\% NP40, 0.5\% sodium dodecyl sulfate, $1 \mathrm{mM}$ phenylmethylsulfonyl fluoride, and $4 \%$ complete inhibitor cocktail, lysates were processed for western blotting. After incubation with the appropriate secondary antibodies, proteinspecific bands were detected and quantified with the Odyssey Infrared Imager (LI-COR; Westburg, Leusden, the Netherlands) or the Chemidoc Imager (Biorad; Veenendaal, the Netherlands) and expressed as fold change to the wild-type control group. 


\section{Statistical analysis}

Differences between groups in levels of each marker examined were tested using one-way ANOVA. Post-hoc Bonferroni correction was used for the comparison of the three groups of interest ( $\mathrm{WtC}$ versus $\mathrm{WtD}, \mathrm{WtD}$ versus $\mathrm{TgD}$ and $\mathrm{WtC}$ versus TgD). We also calculated, and compared between groups, a composite score of GLO-I sensitive oxo-aldehydes (CO and MGO), GO and MGO derived AGEs (CML, CEL, MH-H1, THP and AP) and ROS (MDA, 8isoprostane and nitrotyrosine) by averaging the z-scores of each of the respective markers. Each z-score represents the distance between the raw score from the total mean in units of the standard deviation (assuming negative values when the raw score is below the mean, and positive when above), thus enabling the combination of several markers originally expressed as different units. The three composite scores thus obtained represent a good integral measure of the processes/mechanisms they represent and have the advantage of reducing the influence of the biological variability when each of their constituent markers are tested separately. To enable a meaningful interpretation of the magnitude of the differences in these composite scores, comparisons between the TG diabetic group and the referent group (WT) were also expressed relative to the levels in this group (i.e. as percentage). A p-value of less than 0.05 was considered statistically significant. Data are expressed as the mean \pm SEM.

\section{Results}

\section{Glyoxalase-I overexpression in the transgenic GLO-I rats}

GLO-I activity was significantly elevated in multiple tissues of the GLO-I transgenic rats including heart, kidney, eye, brain, aorta and gastrocnemius muscle as compared with their wild type (WT) littermates (figure 4.1A). In these tissues, human GLO-I protein expression was confirmed by immunoblotting and by immunohistochemical staining as shown for the heart and kidney (figure 4.1B-C). These data demonstrated a functionally active overexpression of GLO-I in the GLO-I transgenic rats.

\section{Baseline and endpoint characteristics}

Table 4.1 presents biochemical data and characteristics of both the non-diabetic and diabetic WT and GLO-I transgenic rats (male rats are depicted in table 4.1a and female rats in 4.1b). Diabetes in both WT and GLO-I transgenic rats, as induced by STZ, resulted in an approximately five-fold increase in blood glucose levels, decreased weight gain and, increased food and fluid intake and urine production without a difference between the WT and the GLO-I transgenic rats. 

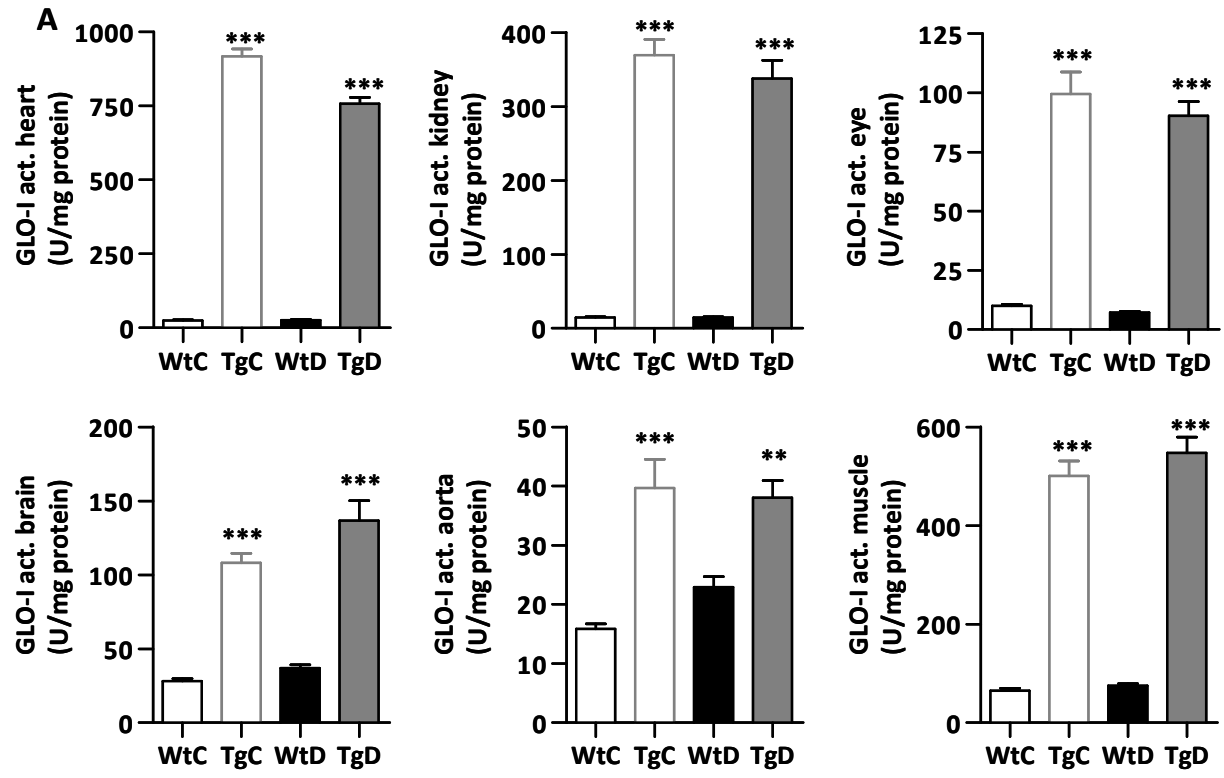

B

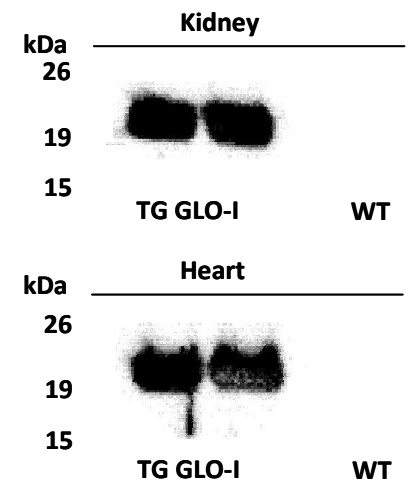

C
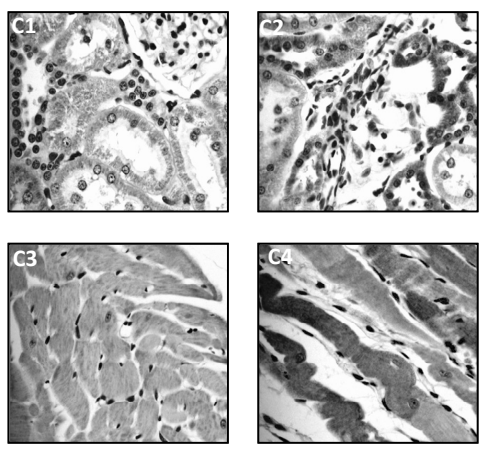

Figure 4.1. GLO-I activity and human protein expression in tissue of wild-type and transgenic GLO-I rats A. GLO-I enzyme activity in lysates of heart, kidney, eye, brain, aorta and gastrocnemius muscle tissue of wildtype control (WtC), transgenic control ( $\mathrm{TgC})$, wild-type diabetic (WtD) and transgenic diabetic (TgD) rats. B. Western blot of human GLO-I protein overexpression in transgenic heart and kidney compared with wild-type heart and kidney lysates. C. Immunohistochemistry of GLO-I protein overexpression in transgenic heart (c2) and kidney (c4) compared with wild-type (c1, c3) (colour figures on page 158). ${ }^{* *} p<0.01$ compared with control, $* * * \mathrm{p}<0.001$ compared with control. 
Table 4.1a. Baseline and endpoint characteristics of the wild type control, GLO-I control, wild type diabetic and GLO-I diabetic male rats.

\begin{tabular}{lccccc}
\hline & & WtC & TgC & WtD & TgD \\
\hline Glucose (mM) & BL & $3.9 \pm 0.5$ & $3.8 \pm 0.4$ & $3.9 \pm 0.2$ & $4.0 \pm 0.4$ \\
& EP & $4.9 \pm 1.2$ & $4.3 \pm 0.6$ & $20.2 \pm 2.1^{* \#}$ & $19.2 \pm 1.5^{* \#}$ \\
\hline Weight (gram) & BL & $308 \pm 35$ & $327 \pm 40$ & $341 \pm 35$ & $310 \pm 27$ \\
& EP & $475 \pm 15^{*}$ & $486 \pm 15^{*}$ & $318 \pm 48^{\#}$ & $318 \pm 23^{\#}$ \\
\hline Food (gram) & BL & $29 \pm 0.9$ & $26 \pm 5.3$ & $27 \pm 2.4$ & $25 \pm 4.0$ \\
& EP & $20 \pm 3.8$ & $18 \pm 1.7$ & $47 \pm 6.3^{* \#}$ & $48 \pm 5.1^{* \#}$ \\
\hline Fluid (mL) & BL & $32 \pm 4.6$ & $30 \pm 5.2$ & $36 \pm 2.7$ & $35 \pm 4.9$ \\
& EP & $27 \pm 6.1$ & $27 \pm 3.5$ & $169 \pm 21.4^{* \#}$ & $246 \pm 66.9^{* \#}$ \\
\hline Urine (mL) & BL & $12 \pm 1.3$ & $13 \pm 3.9$ & $13 \pm 1.1$ & $11 \pm 1.1$ \\
& EP & $19 \pm 4.4$ & $20 \pm 7.6$ & $165 \pm 24.9^{* \#}$ & $205 \pm 19.6^{* \#}$ \\
\hline
\end{tabular}

$* \mathrm{p}<0.05$ compared with baseline. $\# \mathrm{p}<0.05$ compared with control. $\mathrm{BL}=$ Baseline EP = Endpoint

Table 4.1b. Baseline and endpoint characteristics of the wild type control, GLO-I control, wild type diabetic and GLO-I diabetic female rats.

\begin{tabular}{lccccc}
\hline & & WtC & TgC & WtD & TgD \\
\hline Glucose (mM) & BL & $3.9 \pm 0.3$ & $3.9 \pm 0.4$ & $3.8 \pm 0.3$ & $4.0 \pm 0.2$ \\
& EP & $4.5 \pm 0.7$ & $3.8 \pm 0.5$ & $20.3 \pm 3.0^{* \#}$ & $21.9 \pm 4.3^{* \#}$ \\
\hline Weight (gram) & BL & $198 \pm 29$ & $205 \pm 20$ & $203 \pm 17$ & $195 \pm 24$ \\
& EP & $256 \pm 9^{*}$ & $260 \pm 13^{*}$ & $216 \pm 32$ & $212 \pm 8^{\#}$ \\
\hline Food (gram) & BL & $23 \pm 7.4$ & $17 \pm 4.4$ & $17 \pm 1.8$ & $18 \pm 3.0$ \\
& EP & $20 \pm 3.0$ & $23 \pm 2.0$ & $35 \pm 8.1^{* \#}$ & $40 \pm 3.5^{* \#}$ \\
\hline Fluid (mL) & BL & $27 \pm 9.9$ & $27 \pm 2.8$ & $24 \pm 1.8$ & $25 \pm 2.0$ \\
& EP & $28 \pm 4.7$ & $30 \pm 8.0$ & $196 \pm 57.7^{* \#}$ & $163 \pm 12.8^{* \#}$ \\
\hline Urine (mL) & BL & $10 \pm 1.9$ & $11 \pm 1.3$ & $11 \pm 1.0$ & $9 \pm 2.3$ \\
& EP & $18 \pm 2.1$ & $24 \pm 14.2$ & $176 \pm 104.7^{* \#}$ & $114 \pm 35.3^{* \#}$ \\
\hline
\end{tabular}

$* p<0.05$ compared with baseline. $\# p<0.05$ compared with control. $B L=$ Baseline EP = Endpoint

\section{GLO-I overexpression reduces elevated levels of MGO and GO in diabetic rats}

The AGE precursors, MGO, GO and 3-DG, were significantly elevated in the diabetic WT rats as compared with non-diabetic WT rats (figure 4.2 A-C). Levels of the GLO-I- sensitive GO and MGO were normalized in the GLO-I transgenic diabetic rats compared with the WT rats, while 3-DG levels were not influenced by the GLO-I overexpression. The composite score of MGO and GO differed between the WT diabetic and transgenic diabetic group by 1.04. This difference corresponds to a significant $81 \%$ decrease in the composite score of GO and MGO in the GLO-I transgenic diabetic rats compared with the WT diabetic rats $((p<0.05)$; figure 4.2D). In addition, the endproduct of the GLO system, i.e D-lactate, was determined. The plasma levels of D-lactate were elevated in the diabetic rats compared with the non-diabetic rats (figure 4.2E). Also the urine samples showed an increase of Dlactate in the diabetic rats, with a higher concentration of D-lactate in the diabetic GLO-I transgenic rats, indicating a high flux of MGO through the GLO-I pathway (figure 4.2F). 
A
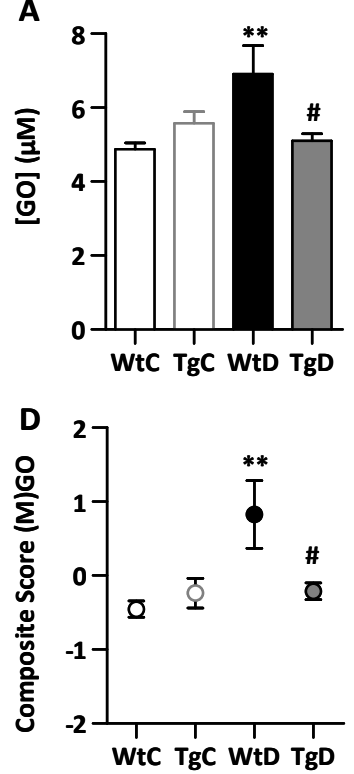

B
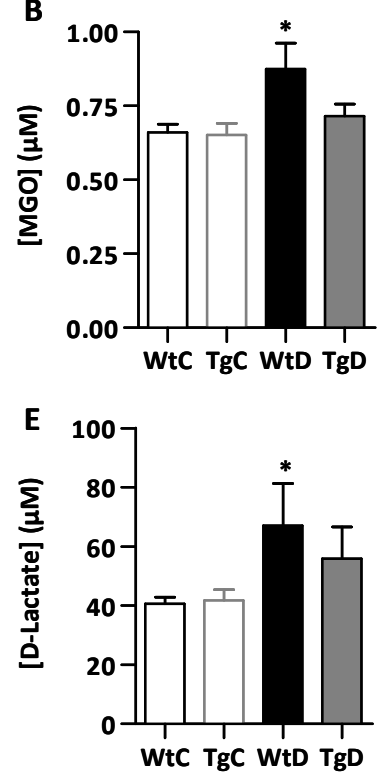

C
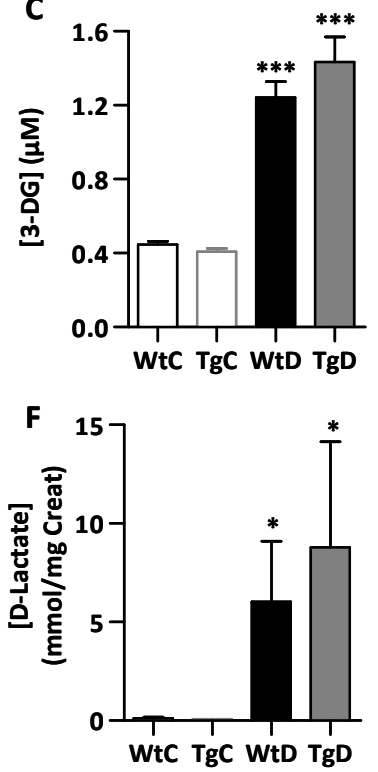

Figure 4.2. Oxo-aldehydes formation and detoxification of (M)GO in the blood of wild-type and transgenic GLO-I rats. Glyoxal (A), MGO (B) and 3DG (C) levels in the blood of wild-type control (WtC), transgenic control $(\mathrm{TgC})$, wild-type diabetic (WtD) and transgenic diabetic (TgD) rats. A composite score was calculated for the GLO-I sensitive oxo-aldehydes $\mathrm{GO}$ and MGO (D). D-lactate levels in the plasma (E) and urine (F) of the wild-type control (WtC), transgenic control (TgC), wild-type diabetic (WtD) and transgenic diabetic (TgD) rats. ${ }^{*} p<0.05$ compared with control, ${ }^{* *} p<0.01$ compared with control, ${ }^{* * *} p<0.001$ compared with control, $\# p<0.05$ compared with wild-type diabetic.

\section{GLO-I overexpression reduces elevated levels of AGEs in diabetic rats}

Next, we evaluated if the overexpression of GLO-I also led to a decreased formation of AGEs. We measured protein-bound levels of CML, CEL, MG-H1, THP and AP in the plasma of the four groups. All AGE adducts were elevated in the plasma of diabetic WT rats compared with non-diabetic WT rats, and this was statistically significant for CML, MG-H1 and AP. There was a trend that the diabetes-induced increase of all AGEs was partially reduced in the diabetic GLO-I transgenic rats as compared with the diabetic WT rats (figure 4.3A-E), although the reduction of the individual AGEs did not reach statistical significance. The absolute difference in the overall composite protein bound AGEs score in the plasma of WT diabetic rats vs. the transgenic diabetic rats was 0.73 and this corresponds to a significant $54 \%$ reduction in the composite score of plasma AGEs in diabetic GLO-I transgenic rats compared with the diabetic WT rats ( $(p<0.05)$; figure 4.3F). 
A
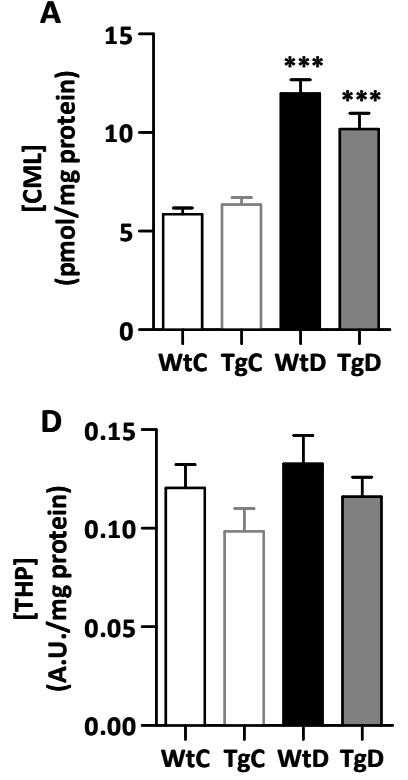

B
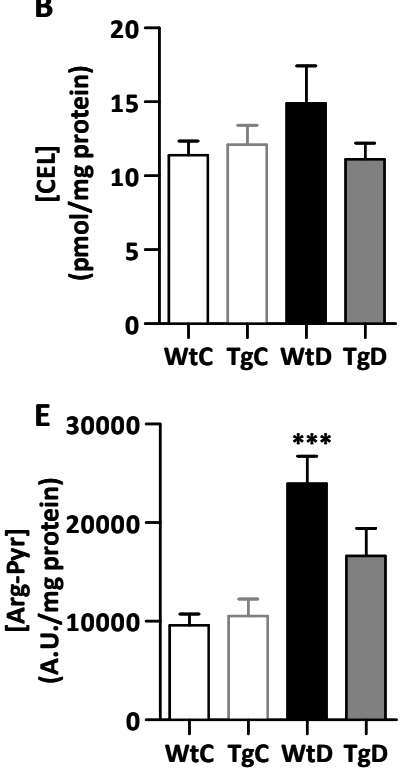

C
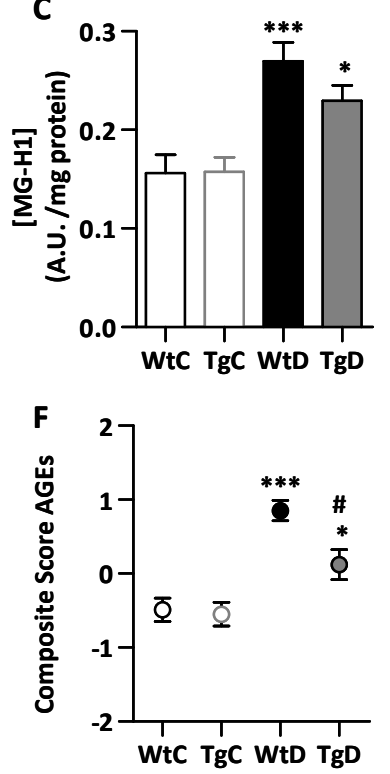

Figure 4.3. Formation of AGEs measured in plasma from wild-type and transgenic GLO-I rats.

CML (A), CEL (B), MG-H1 (C), THP (D) and AP (E) levels were measured with HPLC-MSMS and competitive ELISA in the plasma of the wild-type control $(\mathrm{WtC})$, transgenic control $(\mathrm{TgC})$, wild-type diabetic $(\mathrm{WtD})$ and transgenic diabetic (TgD) rats and in addition a composite score was calculated for the five AGEs (F). ${ }^{*} p<0.05$ compared with control, ${ }^{* * *} \mathrm{p}<0.001$ compared with control, $\# \mathrm{p}<0.05$ compared with wild-type diabetic.

GLO-I overexpression reduces elevated oxidative stress markers in urine and tissue in diabetic rats

Furthermore we examined the effect of GLO-I overexpression on oxidative stress. The urinary excretion of MDA and 8-isoprostane were significantly increased in the diabetic WT rats after 12 weeks of diabetes (figure 4.4A-B). This increase in estimates of oxidative stress was partially prevented in the diabetic GLO-I transgenic rats, although it did not reach statistically significance. In addition, kidney tissue levels of the peroxynitrite marker nitrotyrosine were elevated in the diabetic WT rats, and significantly $(p<0.05)$ attenuated in the diabetic GLO-I transgenic rats (figure 4.4C). Difference in the composite score of these oxidative stress markers between the WT diabetic rats and the transgenic diabetic rats was 0.84 , which corresponds to a significant $51 \%$ decrease in the composite ROS score in the diabetic GLO-I transgenic rats as compared with the diabetic control rats ( $p<0.05$; figure 4.4D). 

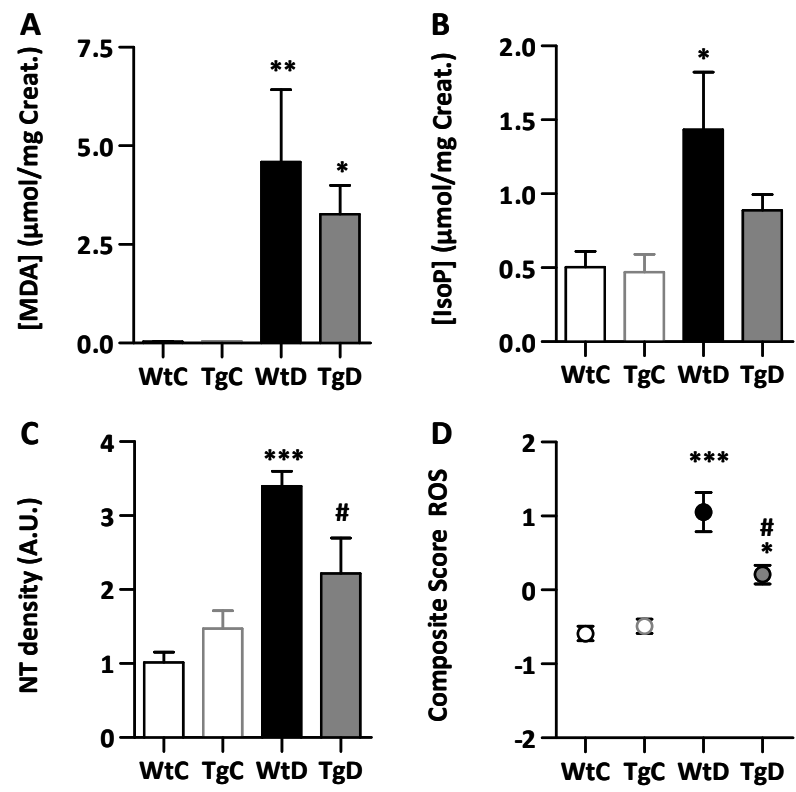

Figure 4.4. Formation of ROS markers in urine and tissue from wild-type and transgenic GLO-I rats.

MDA (A), 8-isoprostane (B) and kidney nitrotyrosine (C) levels in wild-type control (WtC), transgenic control $(\mathrm{TgC})$, wild-type diabetic (WtD) and transgenic diabetic (TgD) rats, were measured with HPLC and westernblotting and a composite score was calculated for the three ROS markers (D).

$* \mathrm{p}<0.05$ compared with control, $* * \mathrm{p}<0.01$ compared with control, $* * * \mathrm{p}<0.001$ compared with control, \# $\mathrm{p}<$ 0.05 compared with wild-type diabetic

\section{Protein levels of mitochondrial oxidative phosphorylation complexes}

To gain more information about the source of the oxidative stress we measured the levels of 4 structural proteins of the complexes of the mitochondrial respiration chain. Complex I, II, III and V of the mitochondrial respiration chain in gastrocnemius muscle lysates were all significantly decreased in the wild type diabetic rats. In the transgenic diabetic rats this decrease was partially counteracted by the GLO-I overexpression. The mitochondrial proteins adenine nucleotide translocator-1 (ANT-1) and uncoupling protein-3 (UCP-3) were not altered by diabetes or GLO-I overexpression, indicating a specific effect on the oxidative phosphorylation complexes (see figure 4.5A-F). The decrease in phosphorylation complexes was accompanied by an increase in the lipid oxidation marker 4-HNE in the muscle from the wild type diabetic rats (figure 4.5G), which was normalized by GLO-I overexpression. 

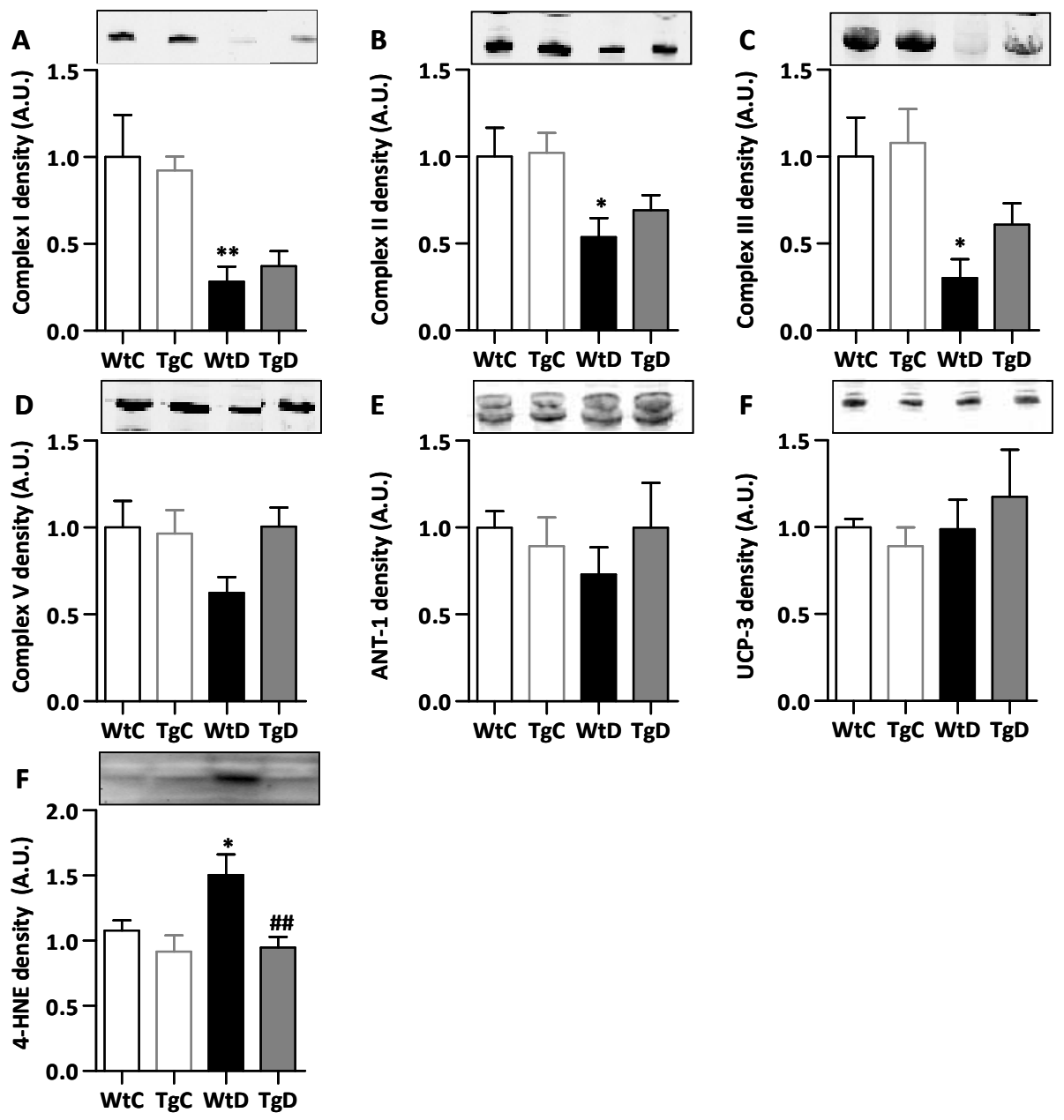

Figure 4.5. Protein levels of mitochondrial proteins and formation of 4-HNE in the muscle of wild type and transgenic GLO-I rats

The mitochondrial proteins Complex I (A), II (B), III (C), V (D), ANT (E), UCP-3 (F) and lipid oxidation marker 4-HNE (G) were measured by Western blotting in gastrocnemius muscle lysates of the wild-type control (WtC), transgenic control (TgC), wild-type diabetic (WtD) and transgenic diabetic (TgD) rats. * $\mathrm{p}<0.05$ compared with control, ${ }^{* *} \mathrm{p}<0.01$ compared with control, \#\# $\mathrm{p}<0.01$ compared with wild-type diabetic.

\section{Discussion}

We report here that GLO-I overexpression in diabetic rats prevents the hyperglycaemiainduced formation of MGO, GO and AGEs, as well as oxidative stress in diabetes.

To demonstrate the importance of GLO-I in the formation of AGEs, transgenic GLO-I rats were generated and increased GLO-I protein levels and activity was confirmed by Western blotting, immunohistochemistry and enzyme activity measurements, respectively. The 
GLO-I activity was at least ten- to fifty-fold higher in GLO-I transgenic rats compared with WT rats in several highly vascularised tissues i.e. heart, kidney, eye, brain, aorta and gastrocnemius muscle.

Our findings are consistent with previous reports. Shinohara et al. transfected endothelial cells with GLO-I, and the hyperglycaemia-induced formation of MGO could be completely normalized by the overexpression of GLO-I, resulting in lower intracellular MGO-derived AGE formation, but not of CML and CEL (6). Consistently, Miyata et al. demonstrated that a deficiency of GLO-I is associated with increased levels of AGEs, further underlining the importance of glyoxalase detoxification system, in particular GLO-I, for the actual level of AGEs (24).

In our study diabetes, as induced by STZ treatment, resulted in increased MGO, GO and 3DG levels. The fact that in our study only the GLO-I substrates MGO and GO are reduced by GLO-I and not 3-DG, which cannot be degraded by GLO-I, underlines the specificity of the GLO-I transgenic model. In addition to a decrease of MGO and GO, we found that the MGO- and/or GO-derived AGEs, MG-H1, THP, AP, CML and CEL were decreased in the plasma of the diabetic GLO-I overexpressing model as compared with WT diabetic rats. Despite the trend towards reduction in all markers, this decrease was only statistically significant when all these markers were comprised into a more robust overall score of AGEs. Therefore interpretations about the effect of GLO-I overexpression on each of the separate AGE adducts should be taken with care. Furthermore, the composite score decrease in total AGE levels was on average only $54 \%$, despite the $81 \%$ reduction of GO and MGO. This only partial reduction in the formation of AGEs in the diabetic transgenic rats might be due to alternative pathways in the formation of AGEs, independent of the precursors MGO and GO and independent of GLO-I expression.

Since the glucose levels were elevated fivefold in the diabetic rats compared with controls, with no effect of GLO-I overexpression, it might be that a significant part of the formation of AGEs is formed via the classical Maillard reaction. In addition, some AGEs, such as CML, can also be formed directly by lipid oxidation and via the myeoloperoxidase reaction, which can explain the GLO-I independent formation of AGEs in diabetes (25).

In addition to the effect on carbonyl stress and AGEs, GLO-I overexpressing also reduced hyperglycaemia-evoked ROS generation, as estimated by three markers of oxidative stress i.e. 8-isoprostane, MDA and nitrotyrosine. 8-Isoprostane is a prostaglandin-like compound generated in vivo by the free-radical-catalyzed peroxidation of arachidonic acid (26). MDA is a bioactive aldehyde generated by extensive oxidation of polyunsaturated fatty acids (27), and nitrotyrosine is formed when the oxidant product of nitric oxide, peroxynitrite, nitrosates tyrosine. Our study shows that these markers of oxidative stress are increased in diabetes and that GLO-I overexpression is capable of lowering these three markers of 
oxidative stress. These data strongly indicate that MGO plays a relevant role in modulating diabetes-induced ROS production, which is in agreement with earlier studies with aminoguanidine and aldose reductase inhibitors (28-30).

Oxidative stress contributes significantly to the development and progression of diabetes and related vascular complications (31). The main source of ROS in diabetes is most probably due to altered mitochondrial metabolism, which results in overproduction of superoxide by the electron-transport chain (32). Rosca and co-workers described that several mitochondrial proteins are susceptible to MGO-dependent glycation, and consequently excessive superoxide is produced due to a decreased electron-transport chain activity (9). In accordance with this study, hyperglycaemia also leads to mitochondrial glycation in C. elegans (13), which was decreased by overexpression of GLO$\mathrm{I}$, indicating the importance of glyoxalase sensitive oxo-aldehydes in the formation of oxidative stress. To further explore the potential source of the oxidative stress in our model, we determined the protein levels of four structural proteins of the mitochondrial electron transport chain complexes. All these complexes were decreased in the diabetic rats, and increased by GLO-I overexpression. This decrease in electron transport chain proteins could lead to deficient mitochondrial function and structure and may contribute to the abnormality of energy metabolism. A consequence of defective mitochondrial function is the increased generation of free radicals which are normally produced as byproducts of oxidative metabolism (33). These free radicals are able to oxidize lipids, proteins, nucleic acids, and other molecules, altering their structure and function (34). Indeed, lipid peroxidation was also measured in the muscle of our diabetic rats, by the elevation of the oxidative stress marker 4-HNE. In the GLO-I overexpression diabetic rats lipid peroxidation was completely prevented, paralleled by an increase in markers of mitochondrial function, suggesting that MGO may affect mitochondrial ROS production via an effect on mitochondrial function.

Pharmaceutical quenching of MGO in vivo is frequently used in the prevention of diabetic complications in animal models. MGO can be trapped by guanidine compounds such as aminoguanidine and metformin, and by vitamin B analogs like pyridoxamine, and can be reduced by the activation of transketolase by thiamine and benfothiamine. Indeed, these compounds lower MGO levels (35). However, since these guanidine compounds are far from specific, the precise contribution of MGO and GO in diabetic complications is still largely unknown. Therefore this new transgenic rat model, which specifically decreases GLO-I sensitive aldehydes such as MGO and GO and their derived AGEs, can provide important knowledge about the contribution of GO, MGO and (M)GO-derived AGEs in the development of diabetic (vascular) complications. Indeed, we recently demonstrated in this model that hyperglycaemia-induced impairment of endothelium-dependent 
vasorelaxation in mesenteric arteries is mediated by intracellular MGO levels (36), demonstrating the usefulness of this model.

In conclusion, our study shows for the first time that GLO-I overexpression decreased GO, MGO, AGEs and ROS levels under in vivo hyperglycaemic conditions. These data are consistent with the link between glycation and oxidative stress. Excessive formation of AGEs and oxidative stress has been implicated in the development of vascular complications in diabetes, and therefore activation of the GLO-I pathway could be an important target in the prevention of diabetes-induced vascular damage.

\section{Acknowledgments}

This study was partially supported by the Dutch Diabetes Foundation (Grant number 2005.11.013). Dr. I Ferreira is supported by a post-doctoral research grant (\#2006T050) from the Netherlands Heart Foundation. Furthermore the authors want to thank Rob Barto (Department of Clinical Chemistry, VU University Medical Center, Amsterdam, the Netherlands), Jean Scheijen and Marjo van de Waarenburg (Department of Internal Medicine, Division of General Internal Medicine, Laboratory for Metabolism and Vascular Medicine, Maastricht University, Maastricht, the Netherlands) for their excellent technical assistance. 


\section{References}

1. Brownlee M: Biochemistry and molecular cell biology of diabetic complications. Nature 414:813-820, 2001

2. Monnier VM, Cerami A: Nonenzymatic browning in vivo: possible process for aging of long-lived proteins. Science 211:491-493, 1981

3. Yaylayan VA, Huyghues-Despointes A: Chemistry of Amadori rearrangement products: analysis, synthesis, kinetics, reactions, and spectroscopic properties. Crit Rev Food Sci Nutr 34:321-369, 1994

4. Beisswenger PJ, Howell SK, Nelson RG, Mauer M, Szwergold BS: Alpha-oxoaldehyde metabolism and diabetic complications. Biochem Soc Trans 31:1358-1363, 2003

5. Thornalley PJ: Glyoxalase l--structure, function and a critical role in the enzymatic defence against glycation. Biochem Soc Trans 31:1343-1348, 2003

6. Shinohara M, Thornalley PJ, Giardino I, Beisswenger P, Thorpe SR, Onorato J, Brownlee M: Overexpression of glyoxalase-I in bovine endothelial cells inhibits intracellular advanced glycation endproduct formation and prevents hyperglycaemia-induced increases in macromolecular endocytosis. J Clin Invest 101:1142-1147, 1998

7. Biswas S, Ray M, Misra S, Dutta DP, Ray S: Selective inhibition of mitochondrial respiration and glycolysis in human leukaemic leucocytes by methylglyoxal. Biochem J 323 ( Pt 2):343-348, 1997

8. Chang T, Wang R, Wu L: Methylglyoxal-induced nitric oxide and peroxynitrite production in vascular smooth muscle cells. Free Radic Biol Med 38:286-293, 2005

9. Rosca MG, Mustata TG, Kinter MT, Ozdemir AM, Kern TS, Szweda LI, Brownlee M, Monnier VM, Weiss MF: Glycation of mitochondrial proteins from diabetic rat kidney is associated with excess superoxide formation. Am J Physiol Renal Physiol 289:F420-430, 2005

10. Miller AG, Smith DG, Bhat M, Nagaraj RH: Glyoxalase I is critical for human retinal capillary pericyte survival under hyperglycemic conditions. J Biol Chem 281:11864-11871, 2006

11. Sakamoto H, Mashima T, Kizaki A, Dan S, Hashimoto Y, Naito M, Tsuruo T: Glyoxalase I is involved in resistance of human leukemia cells to antitumor agent-induced apoptosis. Blood 95:3214-3218, 2000

12. Thornalley PJ, Edwards LG, Kang Y, Wyatt C, Davies N, Ladan MJ, Double J: Antitumour activity of S-pbromobenzylglutathione cyclopentyl diester in vitro and in vivo. Inhibition of glyoxalase I and induction of apoptosis. Biochem Pharmacol 51:1365-1372, 1996

13. Schlotterer A, Kukudov G, Bozorgmehr F, Hutter H, Du X, Oikonomou D, Ibrahim Y, Pfisterer F, Rabbani N, Thornalley P, Sayed A, Fleming T, Humpert P, Schwenger V, Zeier M, Hamann A, Stern D, Brownlee M, Bierhaus A, Nawroth P, Morcos M: C. elegans as model for the study of high glucose mediated lifespan reduction. Diabetes, 2009

14. Inagi R, Miyata T, Ueda Y, Yoshino A, Nangaku M, van Ypersele de Strihou C, Kurokawa K: Efficient in vitro lowering of carbonyl stress by the glyoxalase system in conventional glucose peritoneal dialysis fluid. Kidney Int 62:679-687, 2002

15. McLellan AC, Phillips SA, Thornalley PJ: The assay of S-D-lactoylglutathione in biological systems. Anal Biochem 211:37-43, 1993

16. Schalkwijk CG, Posthuma N, ten Brink HJ, ter Wee PM, Teerlink T: Induction of 1,2-dicarbonyl compounds, intermediates in the formation of advanced glycation end-products, during heat-sterilization of glucose-based peritoneal dialysis fluids. Perit Dial Int 19:325-333, 1999

17. Schindhelm RK, Alssema M, Scheffer PG, Diamant M, Dekker JM, Barto R, Nijpels G, Kostense PJ, Heine RJ, Schalkwijk CG, Teerlink T: Fasting and postprandial glycoxidative and lipoxidative stress are increased in women with type 2 diabetes. Diabetes Care 30:1789-1794, 2007

18. Teerlink T, Barto R, Ten Brink HJ, Schalkwijk CG: Measurement of Nepsilon-(carboxymethyl)lysine and Nepsilon-(carboxyethyl)lysine in human plasma protein by stable-isotope-dilution tandem mass spectrometry. Clin Chem 50:1222-1228, 2004

19. Scheijen JL, van de Waarenburg MP, Stehouwer CD, Schalkwijk CG: Measurement of pentosidine in human plasma protein by a single-column high-performance liquid chromatography method with fluorescence detection. J Chromatogr B Analyt Technol Biomed Life Sci 877:610-614, 2009

20. Marti R, Varela E, Segura RM, Alegre J, Surinach JM, Pascual C: Determination of D-lactate by enzymatic methods in biological fluids: study of interferences. Clin Chem 43:1010-1015, 1997

21. Roest M, Voorbij HA, Van der Schouw YT, Peeters PH, Teerlink T, PG. S: High levels of urinary F2-isoprostanes predict cardiovascular mortality in postmenopausal women J Clin Lipidol 2:298-303 2008

22. van de Kerkhof J, Schalkwijk CG, Konings CJ, Cheriex EC, van der Sande FM, Scheffer PG, ter Wee PM, Leunissen KM, Kooman JP: Nepsilon-(carboxymethyl)lysine, Nepsilon-(carboxyethyl)lysine and vascular cell 
adhesion molecule-1 (VCAM-1) in relation to peritoneal glucose prescription and residual renal function; a study in peritoneal dialysis patients. Nephrol Dial Transplant 19:910-916, 2004

23. Hoeks J, Briede JJ, de Vogel J, Schaart G, Nabben M, Moonen-Kornips E, Hesselink MK, Schrauwen P: Mitochondrial function, content and ROS production in rat skeletal muscle: effect of high-fat feeding. FEBS Lett 582:510-516, 2008

24. Miyata T, van Ypersele de Strihou C, Imasawa T, Yoshino A, Ueda Y, Ogura H, Kominami K, Onogi H, Inagi R, Nangaku M, Kurokawa K: Glyoxalase I deficiency is associated with an unusual level of advanced glycation end products in a hemodialysis patient. Kidney Int 60:2351-2359, 2001

25. Fu MX, Requena JR, Jenkins AJ, Lyons TJ, Baynes JW, Thorpe SR: The advanced glycation end product, Nepsilon-(carboxymethyl)lysine, is a product of both lipid peroxidation and glycoxidation reactions. J Biol Chem 271:9982-9986, 1996

26. Morrow JD, Hill KE, Burk RF, Nammour TM, Badr KF, Roberts LJ, 2nd: A series of prostaglandin F2-like compounds are produced in vivo in humans by a non-cyclooxygenase, free radical-catalyzed mechanism. Proc Natl Acad Sci U S A 87:9383-9387, 1990

27. Requena JR, Fu MX, Ahmed MU, Jenkins AJ, Lyons TJ, Thorpe SR: Lipoxidation products as biomarkers of oxidative damage to proteins during lipid peroxidation reactions. Nephrol Dial Transplant 11 Suppl 5:48-53, 1996

28. Ihm SH, Yoo HJ, Park SW, Ihm J: Effect of aminoguanidine on lipid peroxidation in streptozotocin-induced diabetic rats. Metabolism 48:1141-1145, 1999

29. Kedziora-Kornatowska K, Luciak M: Effect of aminoguanidine on lipid peroxidation and activities of antioxidant enzymes in the diabetic kidney. Biochem Mol Biol Int 46:577-583, 1998

30. Drel VR, Pacher P, Stevens MJ, Obrosova IG: Aldose reductase inhibition counteracts nitrosative stress and poly(ADP-ribose) polymerase activation in diabetic rat kidney and high-glucose-exposed human mesangial cells. Free Radic Biol Med 40:1454-1465, 2006

31. Ceriello A: Controlling oxidative stress as a novel molecular approach to protecting the vascular wall in diabetes. Curr Opin Lipidol 17:510-518, 2006

32. Nishikawa T, Edelstein D, Du XL, Yamagishi S, Matsumura T, Kaneda Y, Yorek MA, Beebe D, Oates PJ, Hammes HP, Giardino I, Brownlee M: Normalizing mitochondrial superoxide production blocks three pathways of hyperglycaemic damage. Nature 404:787-790, 2000

33. Rabbani N, Thornalley PJ: Dicarbonyls linked to damage in the powerhouse: glycation of mitochondrial proteins and oxidative stress. Biochem Soc Trans 36:1045-1050, 2008

34. Kakkar P, Singh BK: Mitochondria: a hub of redox activities and cellular distress control. Mol Cell Biochem 305:235-253, 2007

35. Reddy VP, Beyaz A: Inhibitors of the Maillard reaction and AGE breakers as therapeutics for multiple diseases. Drug Discov Today 11:646-654, 2006

36. Brouwers O, Niessen PM, Haenen G, Miyata T, Brownlee M, Stehouwer CD, De Mey JG, Schalkwijk CG: Hyperglycaemia-induced impairment of endothelium-dependent vasorelaxation in rat mesenteric arteries is mediated by intracellular methylglyoxal levels in a pathway dependent on oxidative stress. Diabetologia 53:9891000,2010 
Glyoxalase-I overexpression improves endothelial dysfunction and attenuates renal impairment in diabetic rats

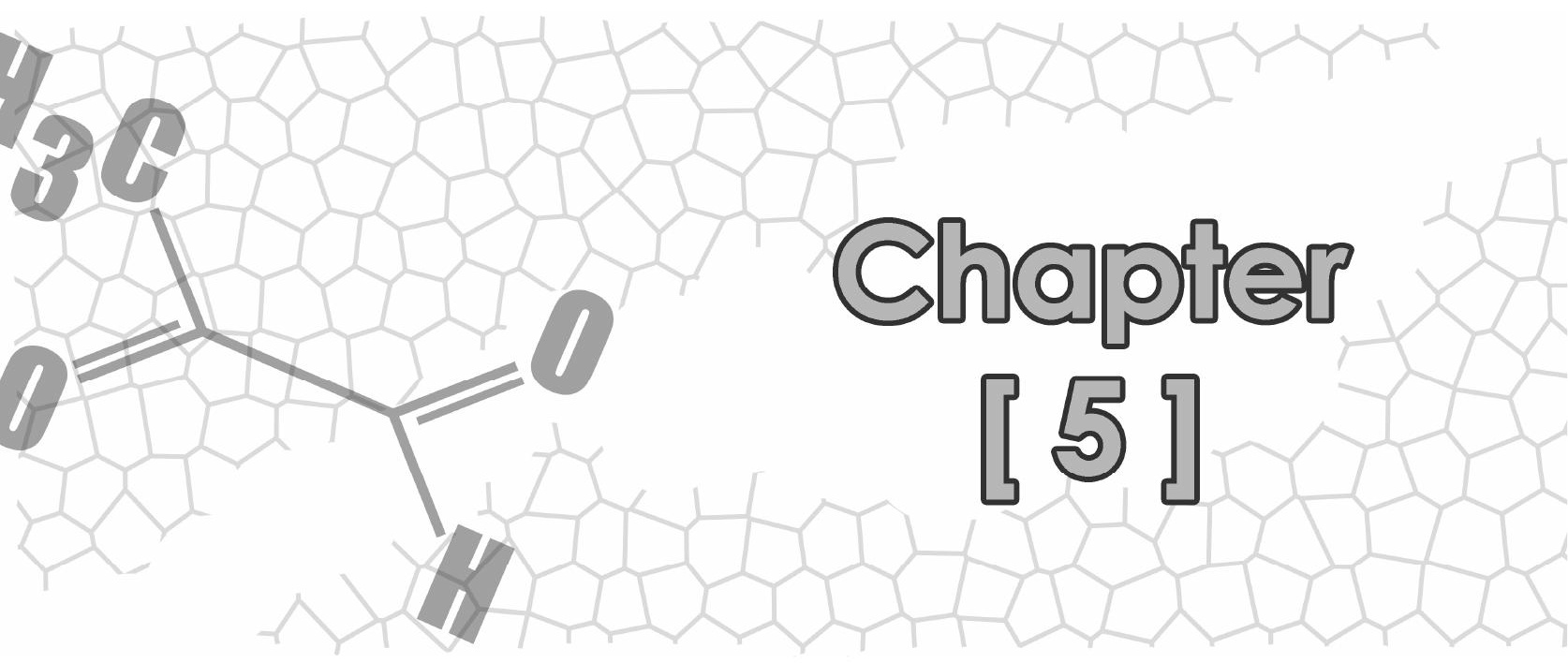

Olaf Brouwers, Petra Niessen, Toshio Miyata, Michael Brownlee, Jo De Mey, Coen Stehouwer and Casper Schalkwijk 


\section{Summary}

Elevated levels of advanced glycation endproducts (AGE) and AGE-precursor methylglyoxal (MGO) are associated with the development of diabetic microvascular complications. In this study glyoxalase-I (GLO-I) transgenic rats were used to explore whether overexpression of this MGO-detoxifying enzyme improves vascular function and prevents nephropathy in a rat model of diabetes.

Wild-type and transgenic rats were made diabetic by streptozocin (STZ) injection and sacrificed after 12 or 24 weeks. Mesenteric arteries were isolated and vascular reactivity was determined after mounting in a wire-myograph. In addition tissues were used for mRNA isolation, protein isolation and fixation in formaldehyde, to measure markers for endothelium dysfunction (VCAM-1, ICAM-1 and eNOS) and glycation (CML and MG-H1). Furthermore urinary nephropathy markers albumin (ALB), osteopontin (OST), lipocalin-2 (LIP) and kidney inflammation molecule-1 (KIM-1) were measured by ELISA.

STZ injection resulted in impaired endothelium-dependent relaxation of mesenteric arteries which was accompanied by an increase in markers of endothelial dysfunction (VCAM-1 and ICAM-1) and glycation (CML and MGH1). This could be prevented by overexpression of GLO-I. In addition urinary nephropathy markers OST, LIP and KIM-1 were increased after 12 weeks, without any effect of GLO-I overexpression. Further increase of the markers after 24 weeks of diabetes was attenuated by the GLO-I intervention. Excretion of ALB was attenuated after both 12 and 24 weeks of diabetes. This study shows that overexpression of GLO-I improves hyperglycaemia-induced endothelium dysfunction and attenuates the further increase in markers for nephropathy after 24 weeks of diabetes. 


\section{Introduction}

Comprehensive studies emphasized that hyperglycaemia is an independent risk factor for microvascular complications leading to excess morbidity and mortality observed in diabetic patients (1-3). Microangiopathy, damage to the small blood vessels and capillaries, is a direct result of chronic hyperglycaemia and the main course of major clinical microvascular complications, such as retinopathy and nephropathy $(4 ; 5)$. In the pathogenesis of microvascular complications, dysfunction of the vascular endothelium is considered not only as an important factor in its initiation, but also in the progression and clinical sequelae (6). One hypothesis about how hyperglycaemia leads to endothelial dysfunction and consequently vascular complications is the formation of advanced glycation endproducts (AGEs) (7). AGEs are a heterogeneous family of non-enzymatically modified proteins, which are increased in patients with diabetes. In addition to the formation of AGEs by carbohydrates in the classical Maillard reaction, intracellular glucose-derived glycolytic intermediates such as methylglyoxal (MGO), glyoxal (GO) and 3deoxyglucocone (3-DG) are also known to form AGEs (8; 9). Because these intracellular dicarbonyl compounds form much more glycated proteins and at a faster rate than do equimolar amounts of glucose, intracellular AGE formation has gain an important role in the link between AGEs and diabetic complications.

Indeed, it has been shown that intracellular AGEs are implicated in activating intracellular signalling pathways as well as in modifying the function of intracellular proteins, thereby contributing to diabetic vascular complications (10). The highly reactive dicarbonyl compound MGO has been identified as the major precursor in the formation of intracellular AGEs in endothelial cells (11). MGO is formed mainly by the non-enzymatic and enzymatic fragmentation of the triose phosphates glyceraldehyde-3-phosphate and dihydroxy-acetone-phosphate and is, under physiological circumstances, efficiently detoxified by the glyoxalase system, in which glyoxalase-I (GLO-I) is the rate limiting step (12). MGO is known to have detrimental effects on cellular function (13). It is also evident that elevated levels of MGO are responsible for renal oxidative stress as demonstrated in diabetic rats (14). Most importantly, also progression of hypertension and diabetic nephropathy in humans is significantly related to increased levels of MGO $(15 ; 16)$.

We previously showed that GLO-I overexpression in diabetic rats detoxifies MGO and GO, and thereby decreases AGEs and markers of oxidative stress (17). In addition, we recently demonstrated in an ex vivo model that hyperglycaemia-induced impairment of endothelium-dependent vasorelaxation in rat mesenteric arteries is mediated by intracellular MGO levels (18). However, the involvement of dicarbonyls and in particular the major AGE precursor MGO in endothelial dysfunction and the development of diabetic microvascular complications remains largely unknown. Therefore, the present study explores the effect of GLO-I overexpression on microvascular and renal function in 
streptozotocin (STZ)-induced diabetes. We hypothesize that elevated levels of MGO are involved in microvascular dysfunction and the development of nephropathy, and that GLO-I overexpression can prevent this.

\section{Methods}

\section{Animals}

Young adult wild-type and GLO-I transgenic male rats were made diabetic for a period of 12 weeks by a single intravenous injection of STZ (65 mg/ $\mathrm{kg}$ body weight), weight and agematched control wild-type rats were not injected. In addition also wild-type and GLO-I transgenic male rats were injected with STZ and sacrificed after 24 weeks (dose was 45 $\mathrm{mg} / \mathrm{kg} \mathrm{STZ}$ to prevent severe illness during this long period of hyperglycaemia). Only animals with a fasting blood glucose level above $15 \mathrm{mM}$ were included in the study. The animals were divided into 6 groups: Wild-type control group of 12 weeks ( $12 ; n=5)$, wildtype control group of 24 weeks (C24; $n=9$ ), wild-type STZ-induced diabetic group after 12 weeks of diabetes (D12; n=5), wild-type STZ-induced diabetic group after 24 weeks of diabetes (D24; $n=8)$, GLO-I transgenic STZ-induced diabetic group after 12 weeks of diabetes (DG12; $n=5$ ) and GLO-I transgenic STZ-induced diabetic group after 24 weeks of diabetes (DG24; $n=13$ ).

The rats were housed 2 to 3 per cage in a room with a $12 \mathrm{~h}: 12 \mathrm{~h}$ artificial light cycle (lights on $0700 \mathrm{~h}$ to $1900 \mathrm{~h}$ ), a temperature of $21 \pm 1{ }^{\circ} \mathrm{C}$, and a humidity of $55 \pm 5 \%$. The animals had free access to standard and tap water throughout the experiment. The study complied with Dutch regulations for the care and use of laboratory animals.

At baseline and just before termination, the rats were placed in metabolic cages to collect 24-h urine. Furthermore, also urine production and food and fluid consumption, was monitored at those two time points. Twelve or twenty-four weeks after the induction of diabetes, rats were sacrificed and kidney tissues and EDTA blood were processed for analysis. In all animals, the right and left kidneys were removed and weighed.

\section{Glyoxalase-I activity measurement}

GLO-I activity was assayed by spectrophotometry by monitoring the increase in absorbance at $240 \mathrm{~nm}$ due to the formation of S-D-lactoylglutathione for ten minutes at $37^{\circ} \mathrm{C}$ according to the method by McLellan and Thornalley (19).

\section{Measurement of vascular reactivity}

After sacrificing the rats by exsanguinations, viscera were exposed in order to remove the superior mesenteric vascular arcade which was then pinned onto a dissecting dish containing cold Krebs-Ringer bicarbonate solution (KRB): $118 \mathrm{mM} \mathrm{NaCl} ; 4.7 \mathrm{mM} \mathrm{KCl} ; 1.2$ $\mathrm{mM} \mathrm{KH} \mathrm{PO}_{4} ; 25 \mathrm{mM} \mathrm{NaHCO} ; 1.1 \mathrm{mM} \mathrm{MgSO}$; $2.5 \mathrm{mM} \mathrm{CaCl}$ and $5.0 \mathrm{mM}$ glucose. A 
second order branch of the superior mesenteric artery was cleaned of fat and connective tissue, and a segment of approximately $2.0 \mathrm{~mm}$ in length was removed and mounted in a myograph organ bath (model 610M Danish Myotechnology by J.P. Trading, Denmark) with two steel $40-\mu \mathrm{m}$ wires inserted through the lumen of the segments. After mounting the tissues, the organ bath solution was changed to fresh KRB maintained at $37^{\circ} \mathrm{C}$ and gassed continuously with $95 \% \mathrm{O}_{2}$ and $5 \% \mathrm{CO}_{2}(\mathrm{pH} 7.4)$. The internal diameter of each vessel was normalized as described by Halpern and Mulvany (20). Maximum contraction was measured by incubation with $125 \mathrm{mM} \mathrm{K}^{+}$and $10 \mu \mathrm{M}$ phenylephrine. Endotheliumdependent vasorelaxation was tested with cumulative doses of $A C h(0.001-10 \mu \mathrm{M})$ after precontraction with $10 \mu \mathrm{M}$ phenylephrine.

Specific nitric oxide-(NO)-dependent relaxation was tested after precontraction with 45 $\mathrm{mM} \mathrm{K}{ }^{+}$which blocks endothelium derived hyperpolarizing factors (EDHFs), and preincubation with $10 \mu \mathrm{M}$ indomethacin which inhibits prostacyclins production. In addition to this, endothelium-independent relaxation was tested with the NO-donor SNP $(0.001-10 \mu \mathrm{M})$ after phenylephrine precontraction $(10 \mu \mathrm{M})$.

On paraformaldehyde fixed mesenteric arteries, media cross sectional area, media thickness and lumen diameter were determined by semi-automated morphometry (JAVA 1.21, Jandel Scientific, Corte Madera, CA) on 4- $\mu$ m sections stained with Lawson's solution, a classic elastin stain.

\section{MAb against glyoxalase-I}

Two peptides (211 and 209) based on human GLO-I (Uniprot_ID Q04760) were chosen with Antigen Profiler Software (Thermo Scientific Open Biosystems) for immunization. After a pre-immune control bleed two specific pathogen-free rabbits per antigen were immunized with the synthesized peptide conjugated to keyhole limpet hemocyanin (KLH). Serum was collected from a bleeding on day 35 (after the primary injection and two boosters) and two pooled bleedings on day 56 (after a third booster) and the terminal bleeding at 72 days. Elisa titration confirmed positive immune responses against the two peptides. Western-blot experiments confirmed that the serum 211 recognized both human and rat GLO-I protein and this serum was used in this study.

\section{Immunohistochemistry}

For immunohistochemistry, mesenteric arteries were fixed with phosphate-buffered $(\mathrm{pH}$ 7.4) formaldehyde (4\%) overnight at room temperature. Subsequently, arteries were transferred to $70 \%$ ethanol embedded in paraffin and processed for histological examination.

Anti-GLO-I (1:1.000; homemade), $\mathrm{N}_{\varepsilon}$-(1-carboxymethyl)lysine (CML) (1:4.000; homemade) (21), $\mathrm{N}_{\delta}$-(5-hydro-5-methyl-4-imidazolon-2-yl)-L-ornithine (MG-H1) (1:100.000), and eNOS 
(1:5.000; BD transduction laboratories) were used as primary antibodies. After washing in phosphate buffered saline, pH 7.4 (PBS), sections were incubated for 30 minutes with rabbit anti-mouse biotin-labeled antibody (1:500) at room temperature and subsequently washed in PBS. After incubation with streptavidin-horseradish peroxidase (1:200, Glostrup, Denmark) for 60 minutes at room temperature, peroxidase was visualized with 3,3-diamino-benzidine-tetrahydrochloride $/ \mathrm{H}_{2} \mathrm{O}_{2}$ (Sigma Chemical Company, St. Louis, MO, USA) for 3 to 5 minutes.

Immunoscoring was performed and was scored for anatomical localization and its intensity. For the intensity scoring each positive vessel was given a score of: 0: absent, $1=$ weak positivity; 2 = moderate positivity or 3 = strong positivity. Each multiplication score was then added and the sum was divided by the number of vessels resulting in a immunohistochemical score.

\section{Determination creatinine clearance}

Serum and urinary creatinine concentrations were measured by an automated technique adapted from the method of Jaffé. The creatinine clearance was calculated by the following equation: creatinine clearance (in $\mathrm{mL} /$ minutes) $=$ (urinary creatinine $x$ urinary volume in 24 hours) / (plasma creatinine $\times 24$ hours $\times 60$ minutes).

\section{MSD kit kidney injury}

Rat kidney injury panel 1 was purchased from mesoscale diagnostics (MSD; K15162C-1). This assay detects lipocalin-2, osteopontin, and KIM-1 in a sandwich immunoassay and uses a competitive assay format to detect albumin. All reagents were provided with the MSD kit. Each 96-well plate had 4-carbon electrodes in the bottom of each well, each precoated with one of the 4-anti-kidney injury markers antibodies of interest. After the blocking step, urine samples, standards and controls (premixed with albumin tracer) were added at $50 \mu \mathrm{l}$ per well. The plate was sealed and incubated for $2 \mathrm{~h}$ at room temperature on an orbital shaker (600 rpm). At the end of the incubation the wells were washed three times using $200 \mu \mathrm{l}$ PBS $+0.05 \%$ Tween 20 , soaking for $30 \mathrm{~s}$ and then discarding. Detection antibody was added at $25 \mu \mathrm{l}$ per well, and the plate sealed and incubated for $2 \mathrm{~h}$ at room temperature on an orbital shaker $(600 \mathrm{rpm})$. At the end of the incubation the plate was washed three times as before. $150 \mu \mathrm{l}$ of the MSD Read Buffer was added to each well and the MSD plates were measured on the MSD Sector Imager 2400 plate reader. The raw data was measured as electrochemiluminescence signal (light) detected by photodetectors and analysed using the Discovery Workbench 3.0 software (MSD). A 4parameter logistic fit curve was generated for each analyte using the standards, and the concentration of each sample was calculated. 


\section{Quantitive Real-Time PCR (RT-PCR)}

After homogenisation of vascular tissue by the use of glass beads and a mini beatbeater total cellular RNA was extracted using TRIzol ${ }^{\circledR}$ isolation (Invitrogen). The amounts of RNA extracted were quantified by measuring the absorbance at $260 \mathrm{~nm}$ by spectrophotometry (Nanodrop). Reverse transcription from RNA to DNA was performed with a Reverse Transcriptase kit from Invitrogen under the following conditions: $25^{\circ} \mathrm{C}$ for $10 \mathrm{~min}, 48{ }^{\circ} \mathrm{C}$ for $30 \mathrm{~min}$ and $94{ }^{\circ} \mathrm{C}$ for $30 \mathrm{~s}$. The PCR was performed in a volume of $20 \mu \mathrm{l}$ in each well containing RNA, Quantas Universal PCR MasterMix and a primer of the target, i.e. VCAM1, ICAM-1, eNOS and a primer of the housekeeping gene, ß-actin, all purchased from Eurogentec. Each RT-PCR reaction ran at $50{ }^{\circ} \mathrm{C}$ for $2 \mathrm{~min}, 95^{\circ} \mathrm{C}$ for $10 \mathrm{~min}$ and in 40 cycles changing between $95^{\circ} \mathrm{C}$ for $15 \mathrm{~s}$ and $60{ }^{\circ} \mathrm{C}$ for $1.30 \mathrm{~min}$. Data were analysed with the $\mathrm{ABI}$ Prism 7000 Sequence Detector Software (Applied Biosystems). The output of amplification was measured in the exponential phase of the reaction as the threshold cycle/Ct-value, which is defined as the cycle number at which amplification products are detected, corresponding to the point where fluorescent intensity exceeds the background fluorescent intensity, which is 10 times the standard deviation of the baseline. The relative quantification of target gene was calculated using the formula: (1/2)Ct-target gene- Cthousekeeping gene.

\section{Measurement AGEs}

The AGEs $\mathrm{N}_{\varepsilon}$-(carboxymethyl)lysine (CML) and $\mathrm{N} \varepsilon$-(carboxyethyl)lysine (CEL) were measured in plasma by ultra performance liquid chromatography-tandem mass spectrometry as described previously (22).

\section{Statistics}

All values are expressed as mean \pm S.E.M. Potency $\left(\mathrm{pD}_{2}\right)$ and efficacy (Emax) were calculated from the concentration-response curves to acetylcholine. $\mathrm{pD}_{2}$ was defined as the negative logarithm to base 10 of the $\mathrm{EC}_{50}$ values and Emax was defined as the maximal vasorelaxation to acetylcholine at the highest concentration used and expressed as 100 minus percentage relative contractions to $\mathrm{K}^{+}$. The statistical differences between groups was tested using one-way ANOVA and the shift of the dose-response curve was tested with two-way ANOVA with a post hoc Bonferroni correction for the groups of interest. We also calculated a composite score of urinary nephropathy markers (albumin, lipocalin-2, osteopontin and KIM-1), and circulating AGEs (CML and CEL), by averaging the z-scores of each of the respective markers. Each z-score represents the distance between the raw score from the total mean in units of the standard deviation (assuming negative values when the raw score is below the mean, and positive when above), thus enabling the combination of several markers originally expressed as different units. The two composite 
scores thus obtained represent a good integral measure of the processes/mechanisms they represent and have the advantage of reducing the influence of the biological variability when each of their constituent markers are tested separately. Correlation was assessed by the Pearson correlation coefficient. A p-value of less than 0.05 was considered statistically significant.

\section{Results}

\section{General physical and biochemical characteristics}

The general physical and biochemical characteristics of all rats at termination are summarized in table 5.1 (the results after 12 weeks of diabetes are depicted in table $5.1 \mathrm{a}$ and after 24 weeks of diabetes in table 5.1b). Injection of rats with STZ resulted in characteristic symptoms of diabetes including; decreased body weight gain, hyperglycaemia, hyperphagia, polydipsia and polyuria (for all; $p<0.05$ ). After 24 weeks of diabetes, glucose levels were significantly higher then after 12 weeks of diabetes $(p<0.05)$. GLO-I overexpression in the transgenic diabetic rats did not influence the hyperglycaemic state and weight of the animals nor the, hyperphagia, polydipsia and polyuria after both periods of hyperglycaemia.

Table 5.1a. General physical and biochemical characteristics of the rats after 12 weeks of diabetes.

\begin{tabular}{lccc}
\hline & Control & Diabetic & Diabetic GLO-I \\
\hline Body weight $(\mathrm{g})$ & $486 \pm 8$ & $309 \pm 12^{*}$ & $321 \pm 13^{*}$ \\
Glucose $(\mathrm{mM})$ & $5 \pm 1$ & $21 \pm 2^{*}$ & $19 \pm 1^{*}$ \\
Food intake $(\mathrm{g})$ & $20 \pm 2$ & $47 \pm 4^{*}$ & $48 \pm 2^{*}$ \\
Fluid intake $(\mathrm{mL})$ & $27 \pm 3$ & $169 \pm 12^{*}$ & $226 \pm 14^{*}$ \\
Urine production $(\mathrm{mL})$ & $19 \pm 2$ & $165 \pm 14^{*}$ & $213 \pm 5^{*}$ \\
\hline
\end{tabular}

$\mathrm{p}<0.05$ compared with controls.

Table 5.1b. General physical and biochemical characteristics of the rats after 24 weeks of diabetes.

\begin{tabular}{lccc}
\hline & Control & Diabetic & Diabetic GLO-I \\
\hline Body weight $(\mathrm{g})$ & $475 \pm 12$ & $300 \pm 11^{*}$ & $300 \pm 12^{*}$ \\
Glucose $(\mathrm{mM})$ & $6 \pm 1$ & $32 \pm 1^{* \$}$ & $31 \pm 1^{* \$}$ \\
Food intake $(\mathrm{g})$ & $19 \pm 2$ & $43 \pm 2^{*}$ & $43 \pm 2^{*}$ \\
Fluid intake $(\mathrm{mL})$ & $19 \pm 2$ & $187 \pm 14^{*}$ & $200 \pm 13^{*}$ \\
Urine production $(\mathrm{mL})$ & $11 \pm 1$ & $183 \pm 15^{*}$ & $207 \pm 11^{*}$ \\
\hline
\end{tabular}

" $p<0.05$ compared with controls, ${ }^{\text {s }} \mathrm{p}<0.05$ compared with 12 weeks.

Morphological analyses of the mesenteric arteries showed that after 12 weeks of diabetes the mesenteric lumen diameter $(132 \pm 2 \mu \mathrm{m}$ vs. $108 \pm 5 \mu \mathrm{m} ; \mathrm{p}<0.05)$, media cross sectional area $\left(10122 \pm 993 \mu \mathrm{m}^{2}\right.$ vs. $\left.7354 \pm 983 \mu \mathrm{m}^{2} ; \mathrm{p}<0.05\right)$, and media thickness $(11.6 \pm 0.9 \mu \mathrm{m}$ vs. $10.2 \pm 0.9 \mu \mathrm{m} ; \mathrm{p}=0.16)$ significantly increased compared with the controls. This vascular hypertrophy was prevented in the diabetic GLO-I overexpression rats $(111 \pm 10 \mu \mathrm{m}$, $7397 \pm 853 \mu \mathrm{m}^{2}$ and $10.1 \pm 0.4 \mu \mathrm{m}$; all $\mathrm{p}<0.05$ compared with D12). However, after 24 weeks 
of hyperglycaemia no differences could be detected in these parameters (see supplement I).

Diabetes-induced AGE formation in rat mesenteric arteries is prevented by glyoxalase-I overexpression

The mesenteric arteries of the GLO-I overexpressing rats showed an increase in GLO-I expression and activity after both 12 and 24 weeks of diabetes as compared with control or wild-type diabetic rats (figure 5.1a). Next, we evaluated if this overexpression of GLO-I also led to a decreased formation of AGEs in mesenteric arteries. After 12 and 24 weeks of diabetes, the immunohistochemical staining of CML (figure 5.1b) and MG-H1 (figure 5.1c) in rat mesenteric arteries were both elevated in the wild-type diabetic group as compared with the control rats, which was statistical significant after 24 weeks of diabetes. Staining for CML and MG-H1 was predominantly localized at the endothelial site, while CML was also present in the smooth muscle cells and adventitia region. Overexpression of GLO-I prevented the diabetes-induced elevation of both $\mathrm{CML}$ and MG-H1 in the diabetic mesenteric arteries ( $p<0.05$ after 24 weeks of diabetes).

Diabetes-induced impairment of vasoreactivity in rat mesenteric arteries is improved by glyoxalase-I overexpression

To determine the function of small resistance arteries in diabetes and the effect of GLO-I overexpression, we measured endothelial-dependent and -independent vasorelaxation in isolated second order mesenteric arteries of the animals in a wire myograph. Maximum contraction did not differ between the groups after 12 weeks (active wall tension in C12, D12 and DG12 was $3.2 \pm 0.4 \mathrm{~N} / \mathrm{m}, 3.2 \pm 0.4 \mathrm{~N} / \mathrm{m}$, and $3.5 \pm 0.4 \mathrm{~N} / \mathrm{m}$ respectively), nor after 24 weeks of diabetes (active wall tension in C24, D24 and DG24 was $3.6 \pm 0.3 \mathrm{~N} / \mathrm{m}, 3.7 \pm 0.3$ $\mathrm{N} / \mathrm{m}$, and $3.3 \pm 0.3 \mathrm{~N} / \mathrm{m}$, respectively). Diabetes resulted in a decrease of both total acetylcholine-induced endothelial-dependent and nitric oxide-(NO)-mediated vasorelaxation after 12 weeks of diabetes, which was even more pronounced after 24 weeks (figure 5.2a and b). SNP-induced endothelium-independent vasorelaxation was not impaired at both time-points (figure 5.2c).

Overall, the decreased endothelium-dependent reactivities in mesenteric arteries of diabetic rats were significantly improved by GLO-I overexpression after both 12 and 24 weeks of diabetes (a table with all Emax and $\mathrm{pD}_{2}$ values can be found in supplement II). 
A

12 weeks
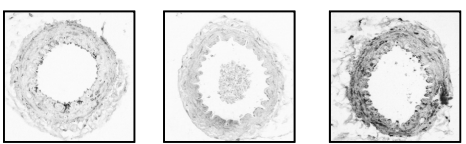

24 weeks

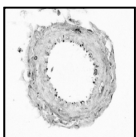

control

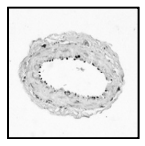

diabetic

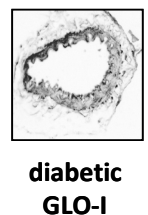

B

12 weeks
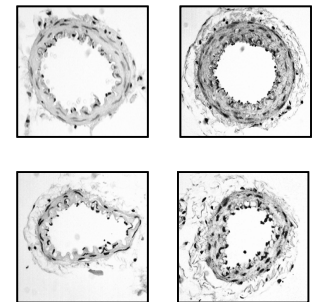

control

C

12 weeks
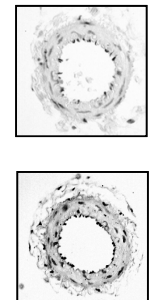

control

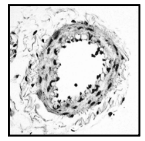

diabetic
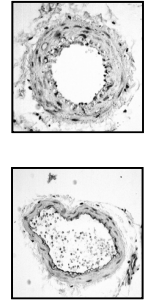

diabetic
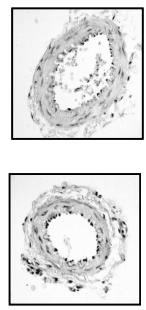

diabetic

GLO-I
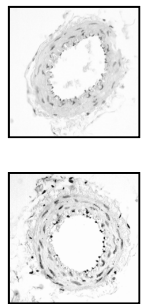

diabetic

GLO-I
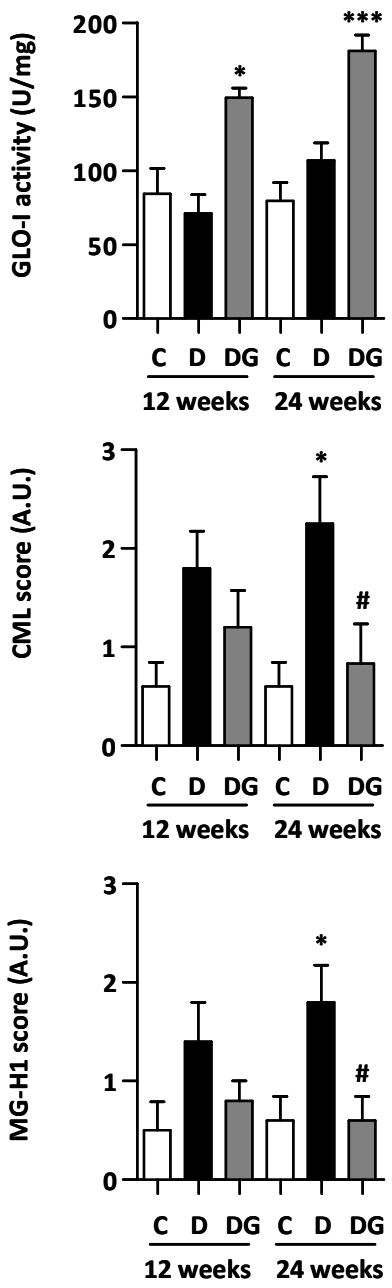

Figure 5.1. GLO-I overexpression prevents diabetes induced vascular glycation.

Vascular glyoxalase-I overexpression and glycation after 12 and 24 weeks of diabetes was addressed by staining isolated second order mesenteric arteries with antibodies against GLO-I, CML or MG-H1. A) Immunohistochemical staining of GLO-I (left) and the GLO-I enzyme activity (right) showed a functionally active overexpression of GLO-I in mesenteric arteries of GLO-I STZ rats (DG) compared with controls (C) $\left(^{*} p<0.05\right.$ compared with control) B). Diabetes (D) resulted in increased levels of CML staining (left) in isolated rat mesenteric arteries after both 12 and 24 weeks of diabetes. Quantification (right) of these stainings showed a statistical significant increase in $\mathrm{CML}$ after 24 weeks of diabetes ( ${ }^{*} \mathrm{p}<0.05$ compared with control), which could be normalized by GLO-I overexpression (\# p<0.05 compared with diabetic). C). Diabetes resulted in increased levels of MG-H1 staining (left) in isolated rat mesenteric arteries after both 12 and 24 weeks of diabetes. Quantification (right) of these stainings showed a statistical significant increase in MG-H1 after 24 weeks of diabetes ( ${ }^{*} p<0.05$ compared with control), which was normalized by GLO-I overexpression (\# $p<0.05$ compared with diabetic) (colour figures on page 159). 

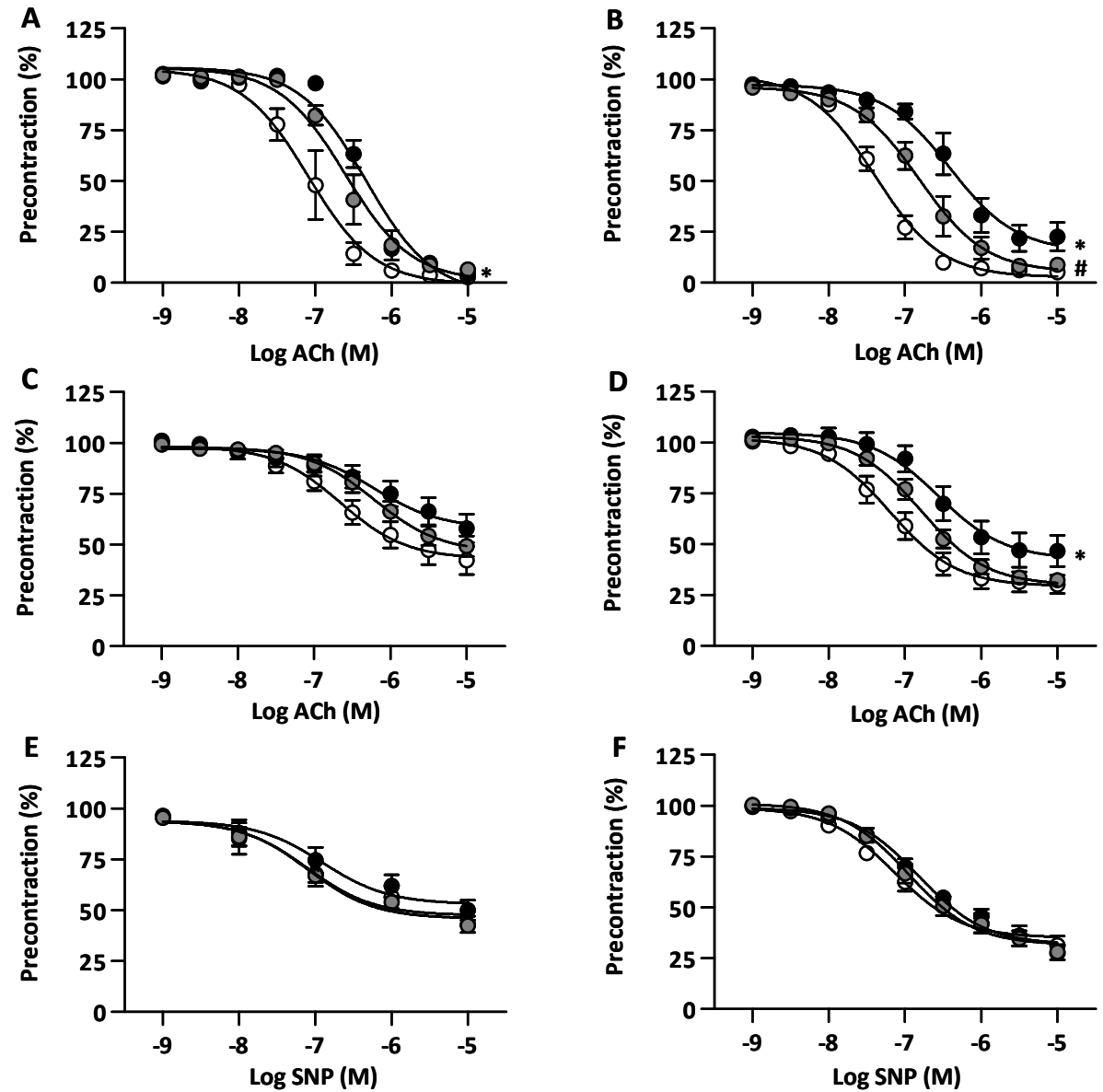

Figure 5.2. Impaired endothelium dependent relaxation is improved by GLO-I overexpression.

Vascular reactivity of isolated rat second order mesenteric arteries after 12 and 24 weeks of diabetes were measured in a wire myograph. In the figures the white, black and grey circles represent the control (C), diabetic (D), and GLO-I diabetic (DG) rats respectively. Both 12 (A) and 24 weeks (B) of diabetes resulted in a statistically significant shift of the dose-response curve to acetylcholine $\left({ }^{*} p<0.05\right.$ compared with control). Overexpression of GLO-I partially prevented this impairment, but the shift of the dose-response curve was only statistical significant after 24 weeks of diabetes (\# $p<0.05$ compared with diabetic). The dose-response curve of the NOmediated vasorelaxation was shifted after both periods ( $C$ and $D)$, but only statistically significant impaired after 24 weeks of diabetes (* $p<0.05$ compared with control). Overexpression of GLO-I resulted in a trend of improvement, but was only borderline significant after 24 weeks of diabetes $(p=0.08)$. Endotheliumindependent SNP-induced vasorelaxation was not altered by neither 12 or 24 weeks of diabetes (E and F). 
A
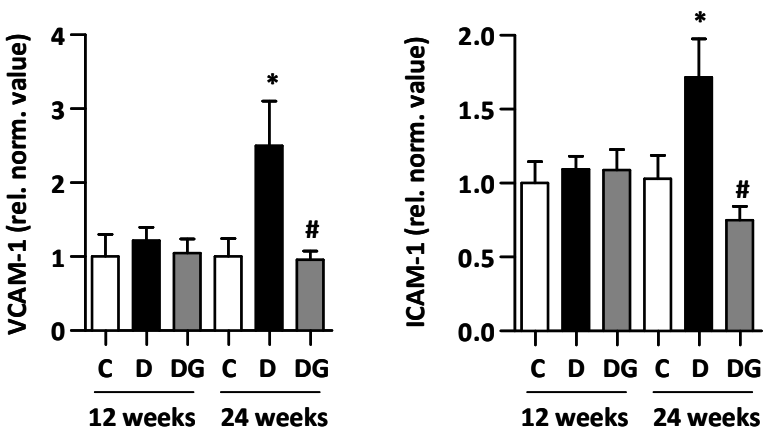

B
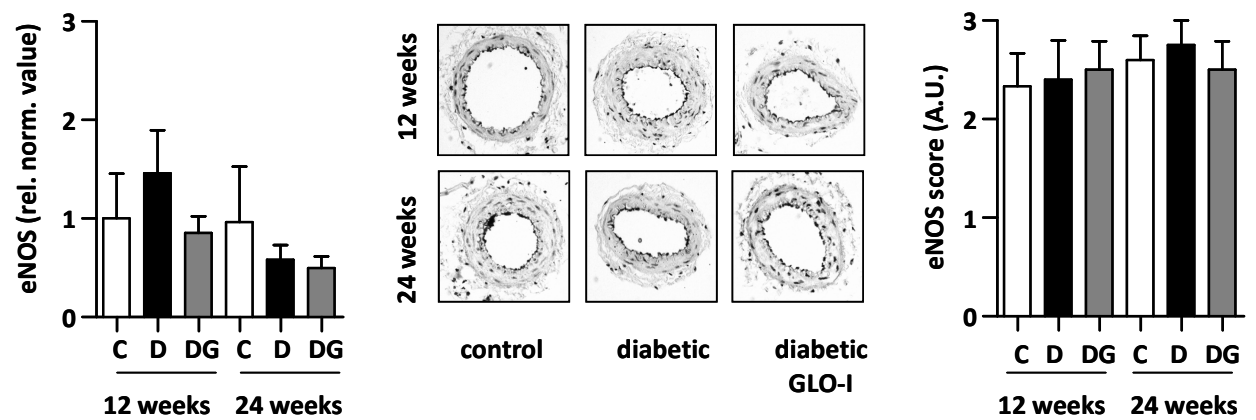

Figure 5.3. Diabetes-induced expression of markers of endothelial dysfunction is prevented by GLO-I overexpression.

Markers of endothelial dysfunction were measured in isolated mesenteric arteries after 12 and 24 weeks of diabetes with real time PCR. A) Both VCAM-1 (left) and ICAM-1 (right) mRNA expression levels were elevated after 24 weeks of diabetes ( ${ }^{*} p<0.05$ compared with control), and GLO-I overexpression prevented this (\# $p<0.05$ compared with diabetic). B). The mRNA expression levels of eNOS (left) were not altered by neither diabetes or GLO-I overexpression. Diabetes and GLO-I overexpression also did not result in altered eNOS protein levels as shown by the immunohistochemical staining (middle) and its quantification (right) (colour figures on page 159).

Diabetes-induced expression of markers of endothelial dysfunction in rat mesenteric arteries is prevented by glyoxalase-I overexpression

We next investigated the effect of diabetes and GLO-I overexpression on other markers of endothelial function. In parallel with the diabetes-induced impairment in vasoreactivity of rat mesenteric arteries, we found increased expression of the endothelial dysfunction markers VCAM-1 and ICAM-1, but only after 24 weeks of diabetes $(p<0.05)$. These increases of mRNA levels in the mesenteric arteries could be completely prevented by GLO-I overexpression $(p<0.05)$ (figure 5.3a). There were no significant effects of diabetes or GLO-I overexpression on eNOS mRNA expression nor protein levels (figure 5.3b). 
Urinary markers of diabetic nephropathy are attenuated after 24 weeks of diabetes by glyoxalase-I overexpression

Results so far demonstrated that diabetes-induced impairment of endothelial function is attenuated by GLO-I overexpression. Because endothelial dysfunction is considered as an important factor in the initiation and progression of vascular complications, we next determined the effect of GLO-I overexpression on diabetic nephropathy. Renal function declined after 12 and 24 weeks of diabetes, resulting in nephromegaly (table 5.2). Furthermore, plasma creatinine levels were increased in the diabetic rats, as a result of the decreased creatinine clearance. The GLO-I overexpression was effective in preventing this decrease in creatinine clearance after 12 weeks (table 5.2a), but not after 24 weeks of diabetes (table 5.2b).

Table 5.2a. Renal characteristics of the rats after 12 weeks of diabetes.

\begin{tabular}{llcc}
\hline & Control & Diabetic & Diabetic GLO-I \\
\hline Left kidney weight $(\mathrm{g})$ & $1.7 \pm 0.1$ & $2.1 \pm 0.1^{*}$ & $2.2 \pm 0.1^{*}$ \\
Right kidney weight $(\mathrm{g})$ & $1.7 \pm 0.1$ & $2.2 \pm 0.2^{*}$ & $2.3 \pm 0.1^{*}$ \\
Left kidney/BW ratio (mg/g) & $3.5 \pm 0.1$ & $6.9 \pm 0.2^{*}$ & $6.7 \pm 0.2^{*}$ \\
Right kidney/BW ratio (mg/g) & $3.5 \pm 0.1$ & $7.2 \pm 0.3^{*}$ & $7.1 \pm 0.1^{*}$ \\
Plasma creatinine (ng/mL) & $3.7 \pm 0.4$ & $4.0 \pm 0.6$ & $3.2 \pm 0.1$ \\
Creatinine clearance (mL/min) & $3.4 \pm 0.4$ & $2.3 \pm 0.3$ & $3.9 \pm 0.2^{\#}$ \\
\hline
\end{tabular}

" $p<0.05$ compared with controls, ${ }^{\#} p<0.05$ compared with diabetics.

Table 5.2b. Renal characteristics of the rats after 24 weeks of diabetes.

\begin{tabular}{|c|c|c|c|}
\hline & Control & Diabetic & Diabetic GLO-I \\
\hline Left kidney weight (g) & $1.4 \pm 0.1$ & $2.0 \pm 0.1^{*}$ & $1.8 \pm 0.1^{*}$ \\
\hline Right kidney weight (g) & $1.5 \pm 0.1$ & $2.0 \pm 0.1^{*}$ & $1.8 \pm 0.1^{*}$ \\
\hline Left kidney/BW ratio (mg/g) & $3.0 \pm 0.1$ & $6.6 \pm 0.2^{*}$ & $6.1 \pm 0.1^{*}$ \\
\hline Right kidney/BW ratio (mg/g) & $3.1 \pm 0.1$ & $6.5 \pm 0.2^{*}$ & $6.1 \pm 0.2^{*}$ \\
\hline Plasma creatinine (ng/mL) & $3.7 \pm 0.1$ & $4.4 \pm 0.2$ & $4.1 \pm 0.2$ \\
\hline Creatinine clearance $(\mathrm{mL} / \mathrm{min})$ & $3.2 \pm 0.1$ & $2.8 \pm 0.3$ & $2.7 \pm 0.2$ \\
\hline
\end{tabular}

p $<0.05$ compared with controls.

Morphological assessment of the kidneys after staining with PAS, Masson trichrome or Sirius red revealed no structural or sclerotic alterations after 24 weeks of diabetes (See supplement III). Therefore, we evaluated levels of early urinary markers of nephropathy in the diabetic animals. Urinary albumin $(p<0.05)$, lipocalin-2 $(p<0.05)$, osteopontin $(p<0.01)$ and KIM-1 $(p<0.01)$ were all elevated after 12 weeks of diabetes. While in the GLO-I diabetic rats this increase remained significant for lipocalin-2, osteopontin and KIM-1, urinary albumin levels were not statically different compared with the control rats (figure 5.4a). After 24 weeks of diabetes a further increase in all four markers of nephropathy was measured in the diabetic rats (for all; $p<0.05$ compared with control). This further increase in three of the four urinary nephropathy markers (albumin, osteopontin and KIM-1) after 24 weeks of diabetes was attenuated by overexpression of GLO-I ( $p<0.05$ ) (figure 5.4b). 

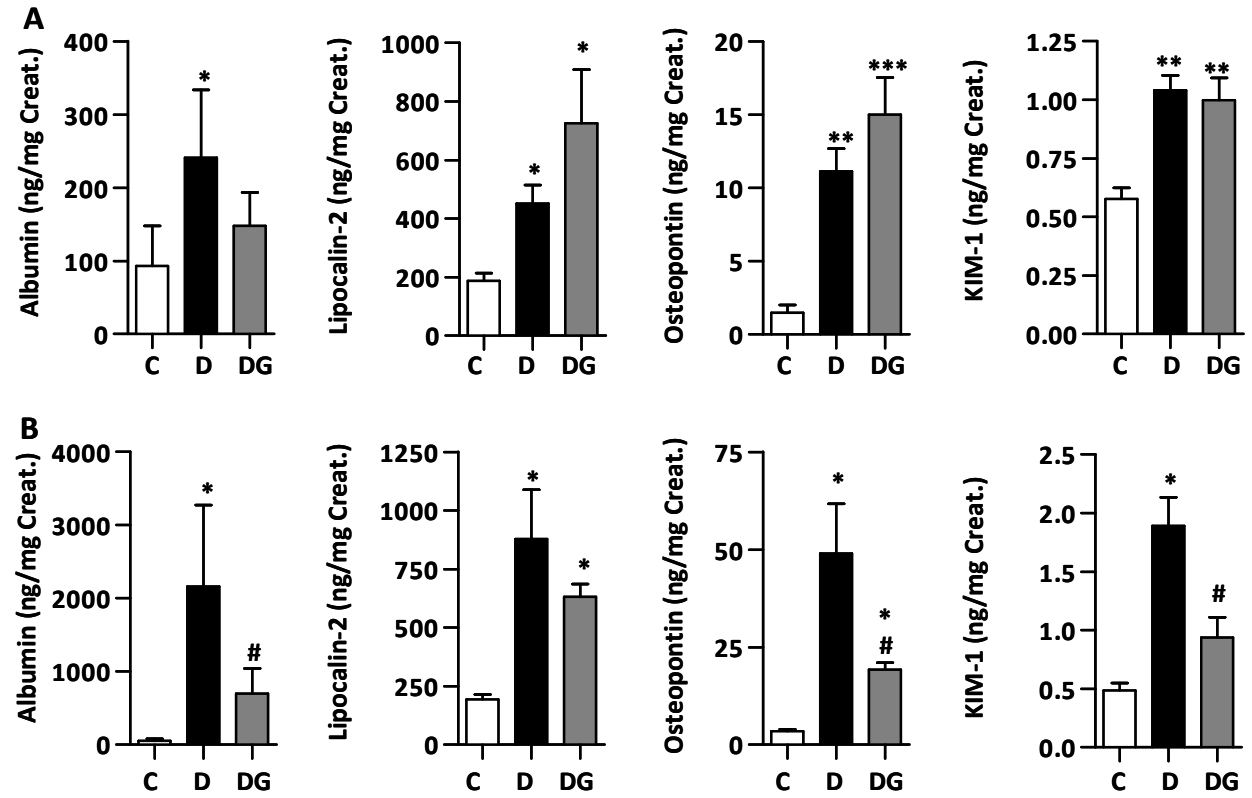

Figure 5.4. Urinary markers of diabetic nephropathy are attenuated by GLO-I overexpression.

Early nephropathy markers were measured in urine after 12 and 24 weeks of diabetes with an ELISA based detection. A). After 12 weeks of diabetes, urinary excretion of albumin (* $p<0.05$ compared with control), lipocalin-2 ( ${ }^{*} \mathrm{p}<0.05$ compared with control), osteopontin (** $\mathrm{p}<0.01$ compared with control) and KIM-1 (*** $\mathrm{p}<0.001$ compared with control) were increased. In the GLO-I STZ rats this increase remained significant except for the urinary albumin levels which were not statically different compared with the control rats. B). After 24 weeks hyperglycaemia further increased the levels of all four the markers (all * $p<0.05$ compared with control), but this elevation was attenuated by GLO-I overexpression for albumin, osteopontin and KIM-1 (all \# p<0.05 compared with diabetes).

\section{Urinary markers of diabetic nephropathy are positively correlated with plasma AGE levels after both 12 and 24 weeks of diabetes}

To determine if the early urinary nephropathy markers are associated with plasma AGE levels, we first calculated a composite score of the four markers of nephropathy. The composite score for albumin, lipocalin-2 osteopontin and KIM-1 was significantly higher in the wild-type diabetic group after both 12 (figure 5.5a) and 24 weeks (figure 5.5b) of diabetes (both $\mathrm{p}<0.001$ compared with control). GLO-I overexpression only significantly decreased ( $p<0.01$ compared with diabetes) this combined nephropathy markers score after 24 weeks of diabetes. Nevertheless after both 12 (figure 5.5c) and 24 weeks (figure $5.5 \mathrm{~d}$ ) of diabetes the nephropathy composite score was strongly and positively correlated (12 weeks, $r=0,561$ and $p<0.05 ; 24$ weeks $r=0,416$ and $p<0.05$ ) with the composite score of circulating CML and CEL measured in the plasma. 

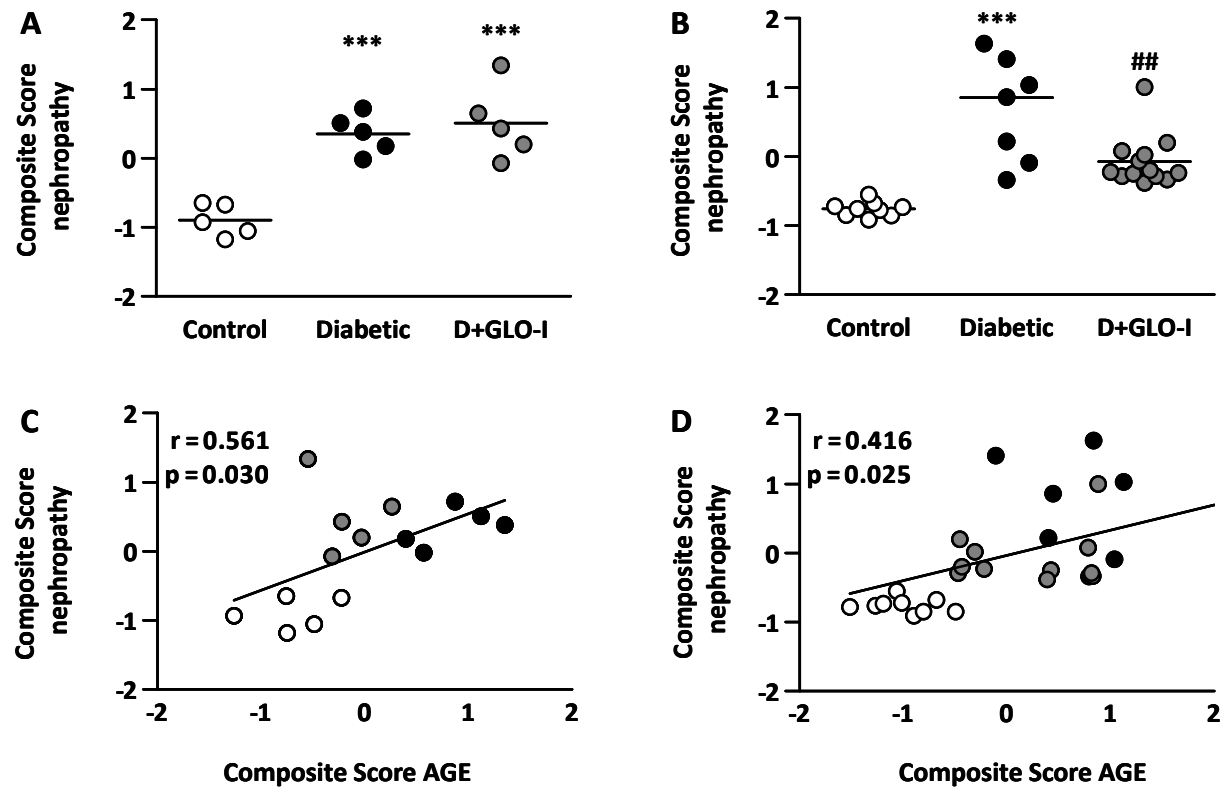

Figure 5.5 Urinary markers of diabetic nephropathy are positively correlated with plasma AGE levels.

Nephropathy makers (albumin, lipocalin-2, osteopontin and KIM-1) measured with the MSD assay kit were combined in a composite score and correlated to a composite score of plasma AGE levels (CML and CEL) measured with UPLC-MSMS. After 12 weeks (A) of diabetes and 24 weeks (B) of diabetes the nephropathy composite score was significantly increased (*** $p<0.001$ compared with control). GLO-I overexpression only decreased these levels after 24 weeks of diabetes (\#\# p<0.01 compared with diabetes). After 12 weeks (C) of diabetes $(r=0,561$ and $p<0.05)$ and 24 weeks $(D)$ of diabetes $(r=0,416$ and $p<0.05)$ the nephropathy composite score was strongly and positively correlated with the composite score of circulating CML and CEL measured in the plasma.

\section{Discussion}

In this study we have shown that the diabetes-induced impairment of vascular function, as measured by an impaired vascular relaxation and an increased expression of markers for endothelial dysfunction in mesenteric arteries of diabetic rats, is improved by overexpression of GLO-I. In addition, overexpression of GLO-I attenuated renal excretion of albumin and other early markers for diabetic nephropathy after 24 weeks of diabetes. These data demonstrated a direct link between increased levels of AGEs, endothelial dysfunction and the development of nephropathy.

Our results show that the diabetes-induced elevation of the major AGES CML and MG-H1 in vascular tissue is prevented by overexpression of GLO-I. This decrease in tissue levels of MGO and GO-derived AGEs nicely demonstrates the efficiency of GLO-I overexpression in the detoxification of both GO and MGO. This is in accordance with our previous study, in which we demonstrated that GLO-I overexpression in diabetic rats prevents elevated 
blood levels of GO and MGO, and thereby decreases plasma AGE levels by approximately $50 \%$ (17). We now demonstrated that also tissue levels of these AGEs are decreased by GLO-I overexpression.

The measurement of endothelium-dependent-relaxation in response to $\mathrm{ACh}$ is a frequently used method to functionally address the state of the endothelium. Several groups have shown that the response to ACh is impaired in diabetes (23-27). In agreement with this, we also observed a decrease in Ach-mediated relaxation, and not by the endothelium independent NO-donor SNP, revealing endothelium dysfunction after both 12 and 24 weeks of diabetes. In addition we also specifically addressed NO-mediated relaxation, by inhibiting prostacyclin and endothelium-dependent hyperpolarizing factor induced relaxation. This approach revealed that the decreased endothelium-dependent relaxation is mainly caused by impairment of the eNOS/NO pathway. Several studies suggest that eNOS is a major target of hyperglycaemia, characterised by a decrease in expression and activity of this enzyme $(28 ; 29)$. However in contrary with these and other studies, our results show that eNOS mRNA and protein levels were not altered by diabetes. This observation indicates a decrease in NO bioavailability rather than insufficient NO production, which is consistent with our earlier observation that MGO and also high glucose incubations of mesenteric arteries decreases NO bioavailability via a pathway dependent on oxidative stress (18). Most importantly, our current research shows that the hyperglycaemia-induced vascular impairment is partially prevented by GLO-I overexpression. However, despite the normalisation of AGE levels, GLO-I overexpression only partially improved endothelium-dependent vasorelaxation. The impaired function, which is still present compared with the control rats, might be explained by other hyperglycaemia-induced pathways like activation of protein kinase $C$, or the hexosamine or polyol pathways (30-32).

Elevated levels of cellular adhesion molecules are associated with an increase in leukocyte adhesion and inflammatory activation (33). Our results show that the STZ induced increases in both VCAM-1 and ICAM-1 gene expression can be prevented by GLO-I overexpression. This result confirms the link between markers of endothelial function and AGEs which is also found in other studies (34-36).

Diabetes is characterised by hypertrophy of several target tissues including the kidneys and the vasculature (37-41). Consistent with these finding, we detected an increase in kidney weight after both 12 and 24 weeks of diabetes. Similar to this, also vascular enlargement in the mesenteric vessels was measured after 12 weeks, but not after 24 weeks. Overexpression of GLO-I prevented the hypertrophy in the vasculature, but did not significantly improve the renal hypertrophy. In our study we did not see any differences in morphological lesions of the glomerulus even after 24 weeks of diabetes, which is in accordance with earlier studies in STZ rats. Hirose et al. described that basal membrane 
thickness is the only parameter for advanced diabetic glomerulopathy which increases significantly in the STZ rats, however only after more than 6 months of diabetes (42). Furthermore Gross et al. (43) highlighted the need of additional models for the study of long term diabetic nephropathy and Bidani et al. (44) speculated that blood pressure depression in STZ rats is one of the reasons for the only modest nephrotic damage observed in the STZ diabetes model.

In humans there is convincing evidence that endothelial dysfunction is closely associated with the development of diabetic nephropathy (45-47). In human type I diabetes, microalbuminuria and macroalbuminuria are accompanied by a variety of markers of endothelial dysfunction, such as increased plasma concentrations of VCAM-1, endothelin1 and plasminogen activator inhibitor-1 (PAI-1), and an impaired endothelium-dependent, nitric-oxide-mediated vasorelaxation (48). Consistent with these studies, we observed an increase in albuminuria after both 12 and 24 weeks. Just like the impaired vascular reactivity, albuminuria was improved by the GLO-I overexpression. In addition to albumin, also two other markers for nephropathy; KIM-1 and osteopontin were improved by GLO-I overexpression. KIM-1 is a tubular protein that was first described by Ichimura et al. (49). KIM-1 is not detectable in normal kidneys, but is one of the proteins that is upregulated, especially at the apical membrane of proximal tubular epithelial cells, in damaged regions after renal injury. Although the function of KIM-1 is unclear, its abundant tubular expression after damage points to a role in either tubular damage or repair. Also osteopontin, a large phosphoglycoprotein adhesion molecule, is upregulated in diabetic nephropathy. A recent study of Nicholas et al. suggests that osteopontin is a key profibrotic factor, which contributes to the development of glomerulosclerosis and albuminuria in diabetes (50).

Diabetic nephrotic injury of both the glomerulus and the tubulointerstitium is accompanied by increased protein excretion and consequently a decline in renal function (51). It is likely that diabetic nephropathy occurs as a result of an interplay of haemodynamic and metabolic factors (52). Thus, endothelial dysfunction, as an early phenomenon, may explain the typical association between albuminuria and extra-renal complications. It is reasonable to speculate that endothelial dysfunction, as we observed in the rat mesenteric arteries, is also present in the intra-renal vasculature $(53 ; 54)$, thereby inducing increases in blood flow and pressure in the glomeruli, which consequently leads to glomeruli dysfunction and urinary protein leakage. Our observations that GLO-I overexpression improves hyperglycaemia-induced vascular- and renal dysfunction, as well as data from other studies, support the involvement of AGEs therein. In STZ-induced diabetes in rats, treatment with the AGE-lowering agents aminoguanidine and pyridoxamine showed improved vascular function and prevented nephropathy, indicating a role of glycation in these pathogenesis $(39 ; 55)$. A study with the 
AGE breaker ALT-711 even showed that the breakdown of already existing AGEs prevents diabetic nephropathy (41). In addition a cohort study by Beisswenger et al. shows that progression of diabetic nephropathy is also significantly related to elevation in dicarbonyl stress in humans (15). Furthermore several cohort studies show that circulating levels of glycation are positively associated with levels of nephropathy, which was also observed in our animal study (56-58).

It is noteworthy to emphasize that we for the first time demonstrated the involvement of intracellular glycation in the development of endothelial dysfunction and diabetic nephropathy in an animal model of diabetes.

In summary, this study shows that overexpression of GLO-I reduces and improves diabetes-induced endothelium dysfunction and attenuates the increase in markers for nephropathy. These observations hint that inhibition of intracellular glycation leads to an improvement of vascular function and amelioration of the impaired kidney function. Activation of GLO-I may provide an approach in preventing endothelial dysfunction and vascular complications in diabetes.

\section{Acknowledgements}

The authors gratefully acknowledge Marjo van den Waarenburg and Jean Scheijen (both Department of Internal Medicine, Division of General Internal Medicine, Laboratory for Metabolism and Vascular Medicine, Maastricht University, Maastricht, the Netherlands) for the measurement of the urinary kidney injury markers and plasma AGE levels respectively.

O.B. researched data and wrote manuscript. P.N. researched data and contributed to discussion. T.M. contributed to discussion and reviewed/edited manuscript. M.B. contributed to discussion and reviewed/edited manuscript. J.M. contributed to discussion and reviewed/edited manuscript. C.D.S. contributed to discussion and reviewed/edited manuscript. C.G.S. wrote manuscript, contributed to discussion and reviewed/edited manuscript. 


\section{Electronic supplementary material}

\section{Supplement I.}

Table la. Morphological characteristics of the rat mesenteric arteries after 12 weeks of diabetes.

\begin{tabular}{lccc}
\hline & Control & Diabetic & Diabetic GLO-I \\
\hline Lumen radius $(\mu \mathrm{m})$ & $108 \pm 5$ & $132 \pm 2^{*}$ & $111 \pm 10^{\#}$ \\
CSA media $\left(\mu \mathrm{m}^{2}\right)$ & $7354 \pm 983$ & $10122 \pm 993^{*}$ & $7397 \pm 853^{\#}$ \\
Media thickness $(\mu \mathrm{m})$ & $10.2 \pm 0.9$ & $11.6 \pm 0.9$ & $10.1 \pm 0.4^{\#}$ \\
M/L ratio & $0.095 \pm 0.006$ & $0.088 \pm 0.006^{*}$ & $0.093 \pm 0.006^{\#}$ \\
\hline
\end{tabular}

" $p<0.05$ compared with controls, ${ }^{\#} p<0.05$ compared with diabetics.

Table Ib. Morphological characteristics of the rat mesenteric arteries after 24 weeks of diabetes.

\begin{tabular}{lccc}
\hline & Control & Diabetic & Diabetic GLO-I \\
\hline Lumen radius $(\mu \mathrm{m})$ & $96 \pm 5$ & $97 \pm 1$ & $107 \pm 7$ \\
CSA media $\left(\mu \mathrm{m}^{2}\right)$ & $5176 \pm 441$ & $5247 \pm 264$ & $6307 \pm 616$ \\
Media thickness $(\mu \mathrm{m})$ & $8.2 \pm 0.4$ & $8.2 \pm 0.4$ & $8.8 \pm 0.4$ \\
M/L ratio & $0.085 \pm 0.006$ & $0.084 \pm 0.004$ & $0.084 \pm 0.004$ \\
\hline
\end{tabular}

\section{Supplement II.}

Table Ila. Vascular reactivity of the rat mesenteric arteries after 12 weeks of diabetes.

\begin{tabular}{lccc}
\hline & Control & Diabetic & Diabetic GLO-I \\
\hline ACh mediated relaxation & & & \\
Potency $\left(\mathrm{pD}_{2}\right)$ & $7.18 \pm 0.15$ & $6.39 \pm 0.09^{*}$ & $6.67 \pm 0.11^{* \#}$ \\
Efficacy $\left(\mathrm{Emax}^{*}\right)(\%)$ & $100.0 \pm 4.4$ & $104.7 \pm 4.6$ & $98.6 \pm 4.5$ \\
\hline NO mediated relaxation & & & \\
Potency $\left(\mathrm{pD}_{2}\right)$ & $6.60 \pm 0.202$ & $6.02 \pm 0.295$ & $6.19 \pm 0.165$ \\
Efficacy $($ Emax) $(\%)$ & $57.5 \pm 4.3$ & $44.2 \pm 6.3$ & $54.0 \pm 4.2$ \\
\hline SNP mediated relaxation & & & $7.10 \pm 0.14$ \\
Potency $\left(\mathrm{pD}_{2}\right)$ & $7.10 \pm 0.27$ & $6.91 \pm 0.29$ & $54.1 \pm 2.4$ \\
\hline Efficacy $\left(\mathrm{Emax}^{*}\right)(\%)$ & $52.5 \pm 4.5$ & $47.0 \pm 4.6$ & \\
\hline
\end{tabular}

" $p<0.05$ compared with controls, " $p<0.05$ compared with diabetics.

Table Ilb. Vascular reactivity of the rat mesenteric arteries after 24 weeks of diabetes.

\begin{tabular}{lccc}
\hline & Control & Diabetic & Diabetic GLO-I \\
\hline ACh mediated relaxation & & & \\
Potency $\left(\mathrm{pD}_{2}\right)$ & $7.39 \pm 0.06$ & $6.41 \pm 0.12^{*}$ & $6.81 \pm 0.09^{* \#}$ \\
Efficacy $(\mathrm{Emax})(\%)$ & $97.3 \pm 1.9$ & $84.7 \pm 4.9$ & $95.8 \pm 3.4$ \\
\hline NO mediated relaxation & & & \\
Potency $\left(\mathrm{pD}_{2}\right)$ & $7.19 \pm 0.108$ & $6.56 \pm 0.176^{*}$ & $6.79 \pm 0.076$ \\
Efficacy (Emax) (\%) & $70.7 \pm 2.7$ & $57.3 \pm 5.0^{*}$ & $70.2 \pm 2.3^{\#}$ \\
\hline SNP mediated relaxation & & & $6.95 \pm 0.08$ \\
Potency $\left(\mathrm{pD}_{2}\right)$ & $7.14 \pm 0.10$ & $6.82 \pm 0.08$ & $68.5 \pm 2.2$ \\
Efficacy (Emax) (\%) & $65.0 \pm 2.5$ & $68.3 \pm 2.3$ &
\end{tabular}

" $p<0.05$ compared with controls, " $p<0.05$ compared with diabetics. 


\section{Supplement III.}

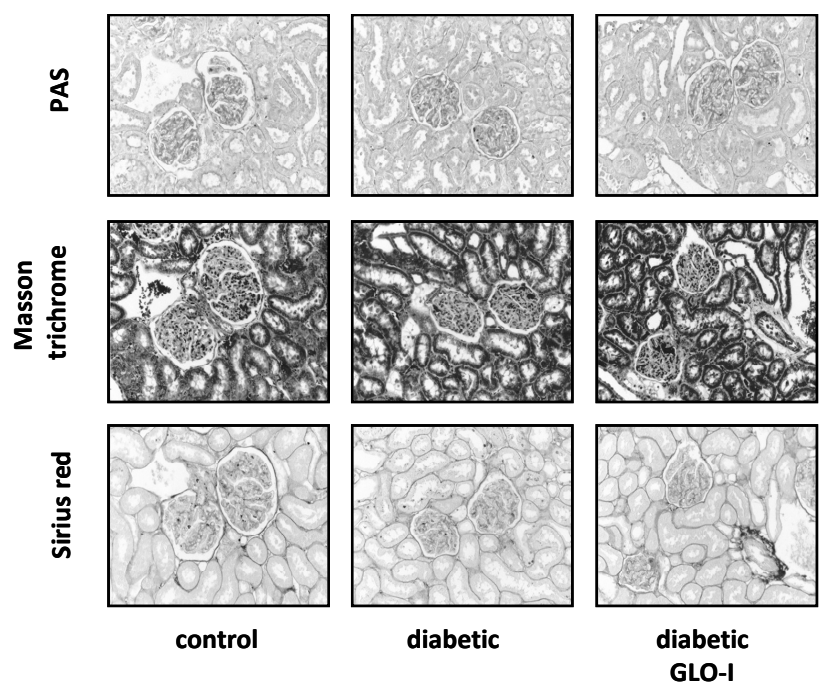

Figure 5.III 24 weeks of diabetes and GLO-I overexpression do not result in structural or sclerotic alterations of kidney morphology.

Structural morphology was assessed in PAS stained kidney sections. Sclerotic damage was visualized by the collagen stainings Masson Trichrome and Sirius Red. The figure shows representive sections of PAS stained, Masson trichrome and Sirius red staining in control, diabetic, and transgenic diabetic rat kidney (colour figures on page 159). 


\section{References}

1. The effect of intensive treatment of diabetes on the development and progression of long-term complications in insulin-dependent diabetes mellitus. The Diabetes Control and Complications Trial Research Group. N Engl J Med 329:977-986, 1993

2. Intensive blood-glucose control with sulphonylureas or insulin compared with conventional treatment and risk of complications in patients with type 2 diabetes (UKPDS 33). UK Prospective Diabetes Study (UKPDS) Group. Lancet 352:837-853, 1998

3. Skyler JS, Bergenstal R, Bonow RO, Buse J, Deedwania P, Gale EA, Howard BV, Kirkman MS, Kosiborod M, Reaven $P$, Sherwin RS: Intensive glycemic control and the prevention of cardiovascular events: implications of the ACCORD, ADVANCE, and VA diabetes trials: a position statement of the American Diabetes Association and a scientific statement of the American College of Cardiology Foundation and the American Heart Association. Circulation 119:351-357, 2009

4. Clark CM, Jr., Lee DA: Prevention and treatment of the complications of diabetes mellitus. N Engl J Med 332:1210-1217, 1995

5. Klein R: Hyperglycaemia and microvascular and macrovascular disease in diabetes. Diabetes Care 18:258-268, 1995

6. Cines DB, Pollak ES, Buck CA, Loscalzo J, Zimmerman GA, McEver RP, Pober JS, Wick TM, Konkle BA, Schwartz BS, Barnathan ES, McCrae KR, Hug BA, Schmidt AM, Stern DM: Endothelial cells in physiology and in the pathophysiology of vascular disorders. Blood 91:3527-3561, 1998

7. Brownlee M: Biochemistry and molecular cell biology of diabetic complications. Nature 414:813-820, 2001

8. Monnier VM, Cerami A: Nonenzymatic browning in vivo: possible process for aging of long-lived proteins. Science 211:491-493, 1981

9. Yaylayan VA, Huyghues-Despointes A: Chemistry of Amadori rearrangement products: analysis, synthesis, kinetics, reactions, and spectroscopic properties. Crit Rev Food Sci Nutr 34:321-369, 1994

10. Brownlee M: The pathobiology of diabetic complications: a unifying mechanism. Diabetes 54:1615-1625, 2005

11. Shinohara M, Thornalley PJ, Giardino I, Beisswenger P, Thorpe SR, Onorato J, Brownlee M: Overexpression of glyoxalase-I in bovine endothelial cells inhibits intracellular advanced glycation endproduct formation and prevents hyperglycaemia-induced increases in macromolecular endocytosis. J Clin Invest 101:1142-1147, 1998

12. Thornalley PJ: The glyoxalase system in health and disease. Mol Aspects Med 14:287-371, 1993

13. Liu BF, Miyata S, Hirota Y, Higo S, Miyazaki H, Fukunaga M, Hamada Y, Ueyama S, Muramoto O, Uriuhara A, Kasuga M: Methylglyoxal induces apoptosis through activation of p38 mitogen-activated protein kinase in rat mesangial cells. Kidney Int 63:947-957, 2003

14. Rosca MG, Mustata TG, Kinter MT, Ozdemir AM, Kern TS, Szweda LI, Brownlee M, Monnier VM, Weiss MF: Glycation of mitochondrial proteins from diabetic rat kidney is associated with excess superoxide formation. Am J Physiol Renal Physiol 289:F420-430, 2005

15. Beisswenger PJ, Drummond KS, Nelson RG, Howell SK, Szwergold BS, Mauer M: Susceptibility to diabetic nephropathy is related to dicarbonyl and oxidative stress. Diabetes 54:3274-3281, 2005

16. Ogawa S, Nakayama K, Nakayama M, Mori T, Matsushima M, Okamura M, Senda M, Nako K, Miyata T, Ito S: Methylglyoxal is a predictor in type 2 diabetic patients of intima-media thickening and elevation of blood pressure. Hypertension 56:471-476

17. Brouwers O, Niessen PM, Ferreira I, Miyata T, Scheffer PG, Teerlink T, Schrauwen P, Brownlee M, Stehouwer CD, Schalkwijk CG: Overexpression of glyoxalase-I reduces hyperglycaemia-induced levels of advanced glycation endproducts and oxidative stress in diabetic rats. the Journal of Biological Chemistry In Press, 2010

18. Brouwers O, Niessen PM, Haenen G, Miyata T, Brownlee M, Stehouwer CD, De Mey JG, Schalkwijk CG: Hyperglycaemia-induced impairment of endothelium-dependent vasorelaxation in rat mesenteric arteries is mediated by intracellular methylglyoxal levels in a pathway dependent on oxidative stress. Diabetologia 53:9891000, 2010

19. McLellan AC, Phillips SA, Thornalley PJ: The assay of S-D-lactoylglutathione in biological systems. Biochem Soc Trans 21:164S, 1993

20. Halpern W, Mulvany MJ: Tension responses to small length changes of vascular smooth muscle cells [proceedings]. J Physiol 265:21P-23P, 1977 
21. Schalkwijk CG, Baidoshvili A, Stehouwer CD, van Hinsbergh VW, Niessen HW: Increased accumulation of the glycoxidation product Nepsilon-(carboxymethyl)lysine in hearts of diabetic patients: generation and characterisation of a monoclonal anti-CML antibody. Biochim Biophys Acta 1636:82-89, 2004

22. Teerlink T, Barto R, Ten Brink HJ, Schalkwijk CG: Measurement of Nepsilon-(carboxymethyl)lysine and Nepsilon-(carboxyethyl)lysine in human plasma protein by stable-isotope-dilution tandem mass spectrometry. Clin Chem 50:1222-1228, 2004

23. Inkster ME, Cotter MA, Cameron NE: Effects of trientine, a metal chelator, on defective endotheliumdependent relaxation in the mesenteric vasculature of diabetic rats. Free Radic Res 36:1091-1099, 2002

24. Makino A, Ohuchi K, Kamata K: Mechanisms underlying the attenuation of endothelium-dependent vasodilatation in the mesenteric arterial bed of the streptozotocin-induced diabetic rat. Br J Pharmacol 130:549556, 2000

25. Ralevic V, Belai A, Burnstock G: Effects of streptozotocin-diabetes on sympathetic nerve, endothelial and smooth muscle function in the rat mesenteric arterial bed. Eur J Pharmacol 286:193-199, 1995

26. Taylor PD, Graves JE, Poston L: Selective impairment of acetylcholine-mediated endothelium-dependent relaxation in isolated resistance arteries of the streptozotocin-induced diabetic rat. Clin Sci (Lond) 88:519-524, 1995

27. Taylor PD, Poston L: The effect of hyperglycaemia on function of rat isolated mesenteric resistance artery. $\mathrm{Br}$ J Pharmacol 113:801-808, 1994

28. Nagareddy PR, Xia Z, MacLeod KM, McNeill JH: N-acetylcysteine prevents nitrosative stress-associated depression of blood pressure and heart rate in streptozotocin diabetic rats. J Cardiovasc Pharmacol 47:513-520, 2006

29. Hink U, Li H, Mollnau H, Oelze M, Matheis E, Hartmann M, Skatchkov M, Thaiss F, Stahl RA, Warnholtz A, Meinertz T, Griendling K, Harrison DG, Forstermann U, Munzel T: Mechanisms underlying endothelial dysfunction in diabetes mellitus. Circ Res 88:E14-22, 2001

30. Booth G, Stalker TJ, Lefer AM, Scalia R: Mechanisms of amelioration of glucose-induced endothelial dysfunction following inhibition of protein kinase $C$ in vivo. Diabetes 51:1556-1564, 2002

31. Musicki B, Kramer MF, Becker RE, Burnett AL: Inactivation of phosphorylated endothelial nitric oxide synthase (Ser-1177) by O-GIcNAc in diabetes-associated erectile dysfunction. Proc Natl Acad Sci U S A 102:1187011875,2005

32. Fortes ZB, Becker C, Oliveira MA, Scivoletto R: Influence of aldose reductase inhibition on the microvascular reactivity in experimental diabetes. Gen Pharmacol 27:917-921, 1996

33. Tailor A, Granger DN: Role of adhesion molecules in vascular regulation and damage. Curr Hypertens Rep 2:78-83, 2000

34. Vlassara H, Fuh H, Donnelly T, Cybulsky M: Advanced glycation endproducts promote adhesion molecule (VCAM-1, ICAM-1) expression and atheroma formation in normal rabbits. Mol Med 1:447-456, 1995

35. Schmidt AM, Hori O, Chen JX, Li JF, Crandall J, Zhang J, Cao R, Yan SD, Brett J, Stern D: Advanced glycation endproducts interacting with their endothelial receptor induce expression of vascular cell adhesion molecule-1 (VCAM-1) in cultured human endothelial cells and in mice. A potential mechanism for the accelerated vasculopathy of diabetes. J Clin Invest 96:1395-1403, 1995

36. Nakamura K, Yamagishi S, Adachi H, Matsui T, Kurita-Nakamura Y, Takeuchi M, Inoue H, Imaizumi T: Serum levels of soluble form of receptor for advanced glycation end products (sRAGE) are positively associated with circulating AGEs and soluble form of VCAM-1 in patients with type 2 diabetes. Microvasc Res 76:52-56, 2008

37. Chang KC, Hsu KL, Tseng CD, Lin YD, Cho YL, Tseng YZ: Aminoguanidine prevents arterial stiffening and cardiac hypertrophy in streptozotocin-induced diabetes in rats. Br J Pharmacol 147:944-950, 2006

38. Chang KC, Liang JT, Tsai PS, Wu MS, Hsu KL: Prevention of arterial stiffening by pyridoxamine in diabetes is associated with inhibition of the pathogenic glycation on aortic collagen. Br J Pharmacol 157:1419-1426, 2009

39. Soulis T, Sastra S, Thallas V, Mortensen SB, Wilken M, Clausen JT, Bjerrum OJ, Petersen H, Lau J, Jerums G, Boel E, Cooper ME: A novel inhibitor of advanced glycation end-product formation inhibits mesenteric vascular hypertrophy in experimental diabetes. Diabetologia 42:472-479, 1999

40. Flyvbjerg A, Landau D, Domene H, Hernandez L, Gronbaek H, LeRoith D: The role of growth hormone, insulinlike growth factors (IGFs), and IGF-binding proteins in experimental diabetic kidney disease. Metabolism 44:6771,1995

41. Forbes JM, Thallas V, Thomas MC, Founds HW, Burns WC, Jerums G, Cooper ME: The breakdown of preexisting advanced glycation end products is associated with reduced renal fibrosis in experimental diabetes. FASEB J 17:1762-1764, 2003 
42. Hirose K, Osterby R, Nozawa M, Gundersen HJ: Development of glomerular lesions in experimental long-term diabetes in the rat. Kidney Int 21:689-695, 1982

43. Gross ML, Ritz E, Schoof A, Adamczak M, Koch A, Tulp O, Parkman A, El-Shakmak A, Szabo A, Amann K: Comparison of renal morphology in the Streptozotocin and the SHR/N-cp models of diabetes. Lab Invest 84:452464, 2004

44. Bidani AK, Picken M, Hacioglu R, Williamson G, Griffin KA: Spontaneously reduced blood pressure load in the rat streptozotocin-induced diabetes model: potential pathogenetic relevance. Am J Physiol Renal Physiol 292:F647-654, 2007

45. Stehouwer CD, Stroes ES, Hackeng WH, Mulder PG, Den Ottolander GJ: von Willebrand factor and development of diabetic nephropathy in IDDM. Diabetes 40:971-976, 1991

46. Astrup AS, Tarnow L, Pietraszek L, Schalkwijk CG, Stehouwer CD, Parving HH, Rossing P: Markers of endothelial dysfunction and inflammation in type 1 diabetic patients with or without diabetic nephropathy followed for 10 years: association with mortality and decline of glomerular filtration rate. Diabetes Care 31:11701176, 2008

47. Stam F, van Guldener C, Schalkwijk CG, ter Wee PM, Donker AJ, Stehouwer CD: Impaired renal function is associated with markers of endothelial dysfunction and increased inflammatory activity. Nephrol Dial Transplant 18:892-898, 2003

48. Stehouwer CD, Lambert J, Donker AJ, van Hinsbergh VW: Endothelial dysfunction and pathogenesis of diabetic angiopathy. Cardiovasc Res 34:55-68, 1997

49. Ichimura T, Bonventre JV, Bailly V, Wei H, Hession CA, Cate RL, Sanicola M: Kidney injury molecule-1 (KIM-1), a putative epithelial cell adhesion molecule containing a novel immunoglobulin domain, is up-regulated in renal cells after injury. J Biol Chem 273:4135-4142, 1998

50. Nicholas SB, Liu J, Kim J, Ren Y, Collins AR, Nguyen L, Hsueh WA: Critical role for osteopontin in diabetic nephropathy. Kidney Int 77:588-600, 2010

51. Mauer SM, Steffes MW, Ellis EN, Sutherland DE, Brown DM, Goetz FC: Structural-functional relationships in diabetic nephropathy. J Clin Invest 74:1143-1155, 1984

52. Cooper ME: Interaction of metabolic and haemodynamic factors in mediating experimental diabetic nephropathy. Diabetologia 44:1957-1972, 2001

53. Dai FX, Diederich A, Skopec J, Diederich D: Diabetes-induced endothelial dysfunction in streptozotocintreated rats: role of prostaglandin endoperoxides and free radicals. J Am Soc Nephrol 4:1327-1336, 1993

54. De Vriese AS, Van de Voorde J, Blom HJ, Vanhoutte PM, Verbeke M, Lameire NH: The impaired renal vasodilator response attributed to endothelium-derived hyperpolarizing factor in streptozotocin--induced diabetic rats is restored by 5-methyltetrahydrofolate. Diabetologia 43:1116-1125, 2000

55. Soulis T, Cooper ME, Vranes D, Bucala R, Jerums G: Effects of aminoguanidine in preventing experimental diabetic nephropathy are related to the duration of treatment. Kidney Int 50:627-634, 1996

56. Lieuw AFML, van Hinsbergh VW, Teerlink T, Barto R, Twisk J, Stehouwer CD, Schalkwijk CG: Increased levels of $\mathrm{N}$ (epsilon)-(carboxymethyl)lysine and $\mathrm{N}$ (epsilon)-(carboxyethyl)lysine in type 1 diabetic patients with impaired renal function: correlation with markers of endothelial dysfunction. Nephrol Dial Transplant 19:631-636, 2004

57. Miura J, Yamagishi S, Uchigata Y, Takeuchi M, Yamamoto H, Makita Z, Iwamoto Y: Serum levels of noncarboxymethyllysine advanced glycation endproducts are correlated to severity of microvascular complications in patients with Type 1 diabetes. J Diabetes Complications 17:16-21, 2003

58. Nin J, Jorsal A, Ferreira I, Schalkwijk C, Prins M, Parving H, Tarnow L, Rossing P, Stehouwer C: Higher Plasma Levels of Advanced Glycation End Products Are Associated With Incident Cardiovascular Disease and All-Cause Mortality in Type 1 Diabetes: A 12-year follow-up study. Diabetes Care 34(2):442-7, 2011. 
Glyoxalase-I overexpression improves blood pressure independently of cardiac function

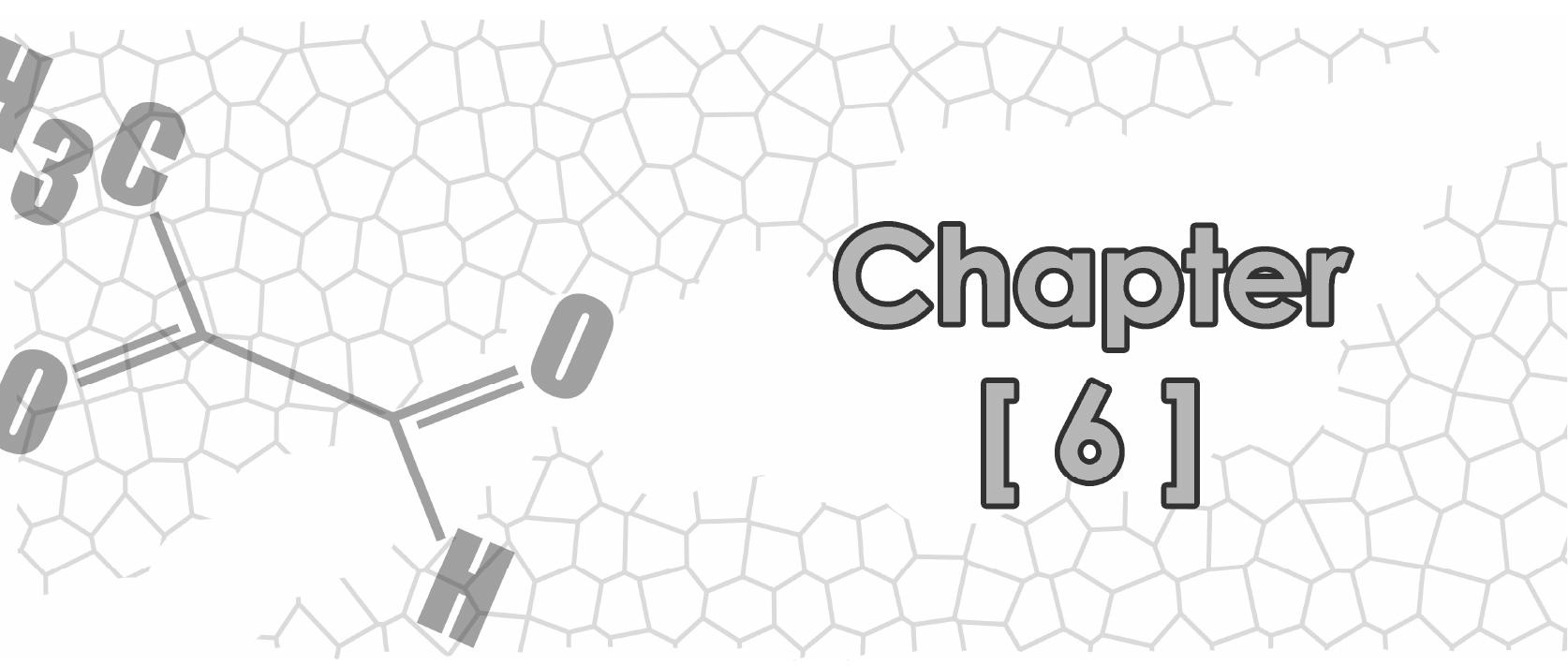

Olaf Brouwers, Petra Niessen, Toshio Miyata, Frans Van Nieuwenhoven, Tom Teerlink, Ben Janssen, Jo De Mey, Coen Stehouwer and Casper Schalkwijk 


\section{Summary}

The reactive advanced glycation endproduct ( $A G E$ ) precursor methylglyoxal (MGO) and MGO-derived AGEs are associated with diabetic vascular complications. In this study glyoxalase-I (GLO-I) transgenic rats were used to explore whether overexpression of this MGO-detoxifying enzyme reduces levels of AGEs, and thereby improves cardiovascular function in a rat model of diabetes.

Diabetes was induced in wild type (WT) and GLO-I transgenic animals by a single tail vein injection with streptozotocin (STZ). After 24 weeks of diabetes, before termination, cardiac function was monitored by ultrasound and mean arterial blood pressure was measured intra-arterially, under isoflurane anaesthesia. After termination, blood was drawn and multiple tissues were collected for further analysis. Urinary oxidative stress marker malondialdehyde (MDA) and circulating levels of glyoxal (GO), MGO, 3deoxyglucosone (3-DG), and the AGEs $\mathrm{N}_{\varepsilon}$-(1-carboxymethyl)lysine (CML), $\mathrm{N}_{\varepsilon}-(1-$ carboxyethyl)lysine (CEL) and $\mathrm{N}_{\delta}$ (5-hydro-5-methyl-4-imidazolon-2-yl)-L-ornithine (MG$\mathrm{H} 1$ ), were assessed by high performance liquid chromatography with fluorescence or tandem mass spectrometry detection. Vascular contraction in the mesenteric and renal bed was measured after mounting the isolated arteries in a wire myograph. STZ treatment resulted in a fivefold increase of blood glucose concentrations irrespective of GLO-I overexpression. Levels of GO, MGO, 3-DG and AGEs were elevated in the diabetic WT rats. Overexpression of GLO-I resulted in decreased levels of GO, MGO, CEL and MG-H1. In WT rats, STZ treatment significantly decreased mean arterial pressure ( $91 \pm 2$ versus $59 \pm 3$ $\mathrm{mmHg} ; \mathrm{p}<0.01)$ and cardiac output $(108 \pm 12$ versus $81 \pm 7 \mathrm{~mL} / \mathrm{min} ; \mathrm{p}<0.05)$. GLO-I overexpression in diabetic rats significantly blunted the fall in mean arterial blood pressure (76 $\pm 7 \mathrm{mmHg} ; \mathrm{p}<0.05$ compared with $\mathrm{WT}$ diabetic rats) without affecting the cardiac output $(80 \pm 7 \mathrm{~mL} / \mathrm{min})$. Vascular contraction was impaired in WT diabetic rats and normalized in the GLO-I transgenic diabetic rats. This study shows that overexpression of GLO-I detoxifies (methyl)glyoxal and thereby decreases (M)GO-derived AGEs in diabetic rats. The fact that GLO-I overexpression is associated with better blood pressures and improved vascular function ex vivo, but not cardiac function, suggests that GLO-I protects arteriolar rather than cardiac function after STZ-induced diabetes in rats. 


\section{Introduction}

Cardiovascular complications are the major cause of morbidity and mortality associated with diabetes (1). Hyperglycaemia is the initial factor leading to dysfunction of vascular cells and consequently haemodynamic changes, although the precise pathways involved remain to be elucidated. The increased formation of advanced glycation endproducts (AGEs) has emerged as one of the potential contributors to the development of diabetic cardiovascular complications (2). One of the major intracellular AGE precursors is the highly reactive dicarbonyl methylglyoxal (MGO). Under physiological circumstances the glyoxalase system, in which the enzyme glyoxalase-I (GLO-I) is the rate-limiting step, efficiently detoxifies MGO to D-lactate (3). However in case of excessive MGO formation due to increased hyperglycaemia-induced glycolysis, GLO-I detoxification is not sufficient and increased levels of MGO and MGO-derived AGEs are associated with vascular complications (4-7).

The injection of streptozotocin (STZ) in rats leads to the development of a clinical syndrome characterised by hyperglycaemia, which is similar to human diabetes. Moreover, the diabetic rats develop the same chronic microvascular complications (neuropathy, nephropathy and retinopathy) as observed in diabetic patients. In addition, this diabetic rat model has also been used to investigate diabetes-induced cardiomyopathy and haemodynamic changes (8). Data on the role of AGEs in this pathogenesis were until now largely derived from studies with the AGE inhibitor aminoguanidine (AG) (9-11). However, the use of AG as an inhibitor of AGE formation is rather aspecific due to the effect of $A G$ on eNOS activity, lipid peroxidation and oxidative stress, independent of MGO scavenging $(12 ; 13)$.

Our group recently described the use of a novel GLO-I overexpressing model in rats to investigate the contribution of GO- and MGO-derived AGEs in diabetes (14). In addition, we also showed that this GLO-I overexpression improves endothelial function and thereby attenuates renal dysfunction in STZ-induced diabetes (Brouwers et al. 2011, submitted). The aim of the current study was to investigate the effects of diabetes on cardiovascular function and the potential beneficial effects of GLO-I overexpression therein.

\section{Methods}

\section{Induction of diabetes and GLO-I overexpression}

All animal studies were carried out in accordance with the guide for the care and use of laboratory animals of the national institutes of health. All experiments involving rats were reviewed and approved by the ethics committee for animal care and use of the Maastricht University, the Netherlands.

Human GLO-I overexpressing rats were bred as described elsewhere (14). Male weight matched Wistar rats were divided in three groups as follows: (i) wild type controls (C), (ii) 
wild type diabetics (D), and (iii) GLO-I transgenic diabetics (DG). Diabetes was induced in animals by a single tail vein injection with $45 \mathrm{mg} / \mathrm{kg} \mathrm{STZ} \mathrm{in} \mathrm{saline} \mathrm{containing} 0.02 \mathrm{M}$ citrate buffer ( $\mathrm{pH} 4.5$ ). After confirmation of hyperglycaemia (5 days later), rats were studied and sacrificed 24 weeks after the induction of diabetes.

\section{Measurement of oxo-aldehydes, AGE and oxidative stress markers}

Blood samples were collected in $11.6 \mathrm{M}$ per-chloric-acid (PCA) and concentrations of the dicarbonyls MGO, GO and 3-DG were determined by reversed-phase high-performance liquid chromatography (HPLC) with gradient elution after derivatization to their respective dimethoxyquinoxaline derivatives. Furthermore, protein-bound $\mathrm{CML}, \mathrm{CEL}$, and MG-H1 were measured in plasma and tissue by HPLC tandem mass spectrometry as described elsewhere (15). Malondialdehyde (MDA) levels were measured in rat urine by HPLC and fluorescent detection.

\section{Measurement of cardiac function and blood pressure}

Rats were anesthetized (isoflurane) and subjected to echocardiography (10 MHz probe; LA14; Esaote Biomedica; IN) just before termination. Left ventricular (LV) free wall thickness during diastole and LV ejection fraction were determined from M-mode images from the short axis of the heart. The ratio of the LV free wall thickness to the diameter of the LV cavity during diastole was used as a measure of eccentric or concentric hypertrophy. From heart rate and differences in internal LV diameter during systole and diastole, stroke volume and cardiac output were calculated, assuming that the ventricular cavity is ellipsoid in shape.

Intra-arterial mean arterial blood pressure was measured under anaesthesia by inserting a pressure tip catheter in the abdominal aorta.

\section{Quantitive Real-Time PCR (RT-PCR)}

Total cellular RNA was extracted from ventricle heart lysates using TRIzol $^{\circledR}$ isolation (Invitrogen). The amounts of RNA extracted were quantified by measuring the absorbance at $260 \mathrm{~nm}$ by spectrophotometry (Nanodrop). Reverse transcription from RNA to DNA was performed with a Reverse Transcriptase kit from Biorad under the following conditions: 25 ${ }^{\circ} \mathrm{C}$ for $10 \mathrm{~min}, 48{ }^{\circ} \mathrm{C}$ for $30 \mathrm{~min}$ and $94^{\circ} \mathrm{C}$ for $30 \mathrm{~s}$. The PCR was performed in a volume of $25 \mu \mathrm{l}$ in each well containing RNA, Quantas Universal PCR MasterMix and primers of the target, i.e. ANP, BNP, CTGF, Col1a1, MMP-2, MMP-9, PAI-1 or TNF $\alpha$ and primers of the housekeeping gene, actin, all purchased from Eurogentec. Each RT-PCR reaction ran at 50 ${ }^{\circ} \mathrm{C}$ for $2 \mathrm{~min}, 95^{\circ} \mathrm{C}$ for $10 \mathrm{~min}$ and in 40 cycles changing between $95^{\circ} \mathrm{C}$ for $15 \mathrm{~s}$ and $60{ }^{\circ} \mathrm{C}$ for $1.30 \mathrm{~min}$. Data were analysed with the ABI Prism 7000 Sequence Detector Software (Applied Biosystems). The output of amplification was measured in the exponential phase 
of the reaction as the threshold cycle/Ct-value, which is defined as the cycle number at which amplification products are detected, corresponding to the point where fluorescent intensity exceeds the background fluorescent intensity, which is 10 times the standard deviation of the baseline. The relative quantification of target gene was calculated using the formula: (1/2)Ct-target gene- Ct-housekeeping gene.

\section{Immunohistochemistry}

For immunohistochemistry hearts were fixed with phosphate-buffered ( $\mathrm{pH}$ 7.4) formaldehyde (4\%) overnight at room temperature. Subsequently, hearts were transferred to $70 \%$ ethanol embedded in paraffin and processed for histological examination. For this, tissue sections of $5 \mu \mathrm{m}$ were fixed at $56^{\circ} \mathrm{C}$ overnight, deparaffinised, rehydrated and stained with haematoxylin and eosin (H\&E) to determine cardiomyocyte cross-sectional surface area using Qwin version 3 morphometric software (Leica, Cambridge, UK). To visualize interstitial fibrosis, the sections were stained with Picro-Sirius Red. The percentage of the LV wall consisting of interstitial collagen was calculated as the ratio of Picro-Sirius-Red positively stained area over total LV tissue area, excluding blood vessels, using Qwin software (Leica).

\section{Measurement of vascular contractile function}

After sacrificing the rats by exsanguinations, viscera were exposed in order to remove the superior mesenteric vascular arcade which was then pinned onto a dissecting dish containing cold Krebs-Ringer bicarbonate solution (KRB): $118 \mathrm{mM} \mathrm{NaCl} ; 4.7 \mathrm{mM} \mathrm{KCl} ; 1.2$ $\mathrm{mM} \mathrm{KH}_{2} \mathrm{PO}_{4} ; 25 \mathrm{mM} \mathrm{NaHCO}_{3} ; 1.1 \mathrm{mM} \mathrm{MgSO}_{4} ; 2.5 \mathrm{mM} \mathrm{CaCl}$ and $5.0 \mathrm{mM}$ glucose. A second order branch of the superior mesenteric artery was cleaned of fat and connective tissue, and a segment of approximately $2.0 \mathrm{~mm}$ in length was removed and mounted in a myograph organ bath (model 610M Danish Myotechnology by J.P. Trading, Denmark) with two steel $40-\mu \mathrm{m}$ wires inserted through the lumen of the segments. After mounting the tissues, the organ bath solution was changed to fresh KRB maintained at $37^{\circ} \mathrm{C}$ and gassed continuously with $95 \% \mathrm{O}_{2}$ and $5 \% \mathrm{CO}_{2}(\mathrm{pH} 7.4)$. In addition a renal artery segment was mounted in a wire myograph. The internal diameter of each vessel was normalized as described by Halpern and Mulvany (16). Maximum contraction was measured by incubation with $125 \mathrm{mM} \mathrm{K}^{+}$and $10 \mu \mathrm{M}$ phenylephrine. For a phenylephrine-induced doseresponse curve concentrations ranging form $1 \mathrm{nM}$ to $10 \mu \mathrm{M}$ were used. In addition, doseresponse curves with increasing amounts of potassium (from $5 \mathrm{mM}$ to $45 \mathrm{mM}$ ) were investigated. 


\section{Statistics}

All values are expressed as mean \pm SEM. The statistical differences between groups was tested using one-way ANOVA with a post-hoc Bonferroni correction for the groups of interest. We also calculated a composite score of blood oxoaldehydes (GO and MGO) and circulating AGEs ( $C M L, C E L$ and $M G-H 1)$, by averaging the z-scores of each of the respective markers. Each z-score represents the distance between the raw score from the total mean in units of the standard deviation (assuming negative values when the raw score is below the mean, and positive when above), thus enabling the combination of several markers originally expressed in different units. The two composite scores obtained represent a good integral measure of the processes/mechanisms they represent and have the advantage of reducing the influence of the biological variability when each of their constituent markers are tested separately. Correlation was assessed by the Pearson correlation coefficient. A p-value of less than 0.05 was considered statistically significant.

\section{Results}

\section{General physical characteristics}

Injection of STZ resulted in glucose levels which were approximately five times higher in the diabetic rats compared with the non-injected control rats, irrespective of GLO-I overexpression (Table 6.1). In addition, the weight of the diabetic animals was significantly decreased compared with control rats and this also was not improved by GLO-I overexpression. Moreover diabetes resulted in increased levels of oxo-aldehydes (3-DG, GO and MGO), AGEs (CML, CEL and MG-H1) and urinary oxidative stress maker MDA. Overexpression of GLO-I normalized GO levels and MGO levels were only mildly, however not statistically significantly, decreased compared with the wild-type diabetic rats. The oxo-aldehyde 3-DG, which is not detoxified by GLO-I, was not altered by the GLO-I overexpression. Only MG-H1, and not $\mathrm{CML}$ and $\mathrm{CEL}$, was statistically significantly decreased by GLO-I overexpression. Both blood pressure and heart rate were significantly decreased in the diabetic rats compared with the control rats. GLO-I overexpression significantly prevented the fall in blood pressure, but did not statistically influence the heart rate of the animals (see Table 6.1). The Pearson correlation score showed a strong negative correlation between dicarbonyls or AGEs levels and mean arterial blood pressure (see figure 6.1). 

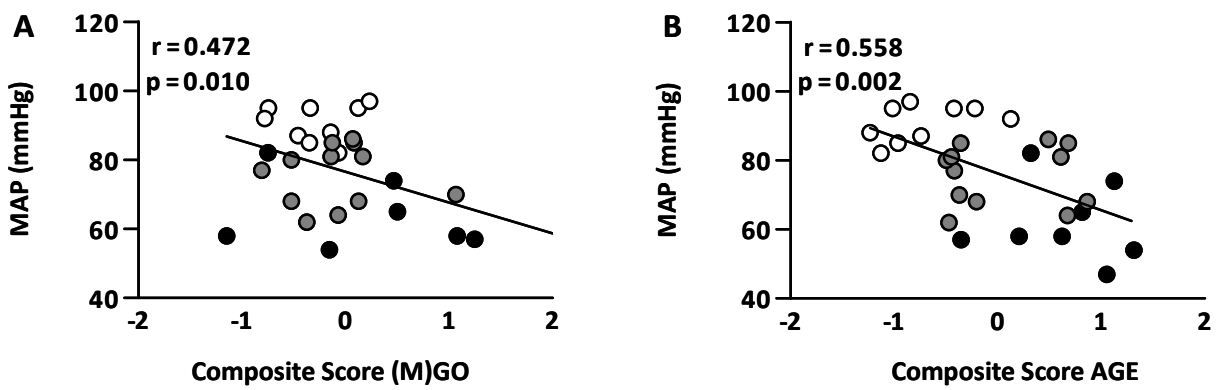

Figure 6.1. Correlation between oxoaldehydes, AGEs and mean arterial pressure (MAP). The white circles are the control rats, the black circles the wild-type diabetics and the grey circles are the diabetic rats with a GLO-I overexpression. A. The composite Z-score of blood GO and MGO is strongly negatively correlated with blood pressure $(r=-0,472 ; p=0.010)$. B. The composite $Z$-score of plasma CML, CEL and MG-H1 is strongly negatively correlated with blood pressure $(r=-0,558 ; p=0.002)$.

Table 6.1. Effect of GLO-I overexpression on general physical and biochemical characteristics in rats after 24 weeks of STZ.

\begin{tabular}{lccc}
\hline & Control & Diabetic & Diabetic GLO-I \\
\hline Body weight (g) & $475 \pm 12$ & $300 \pm 11^{*}$ & $300 \pm 12^{*}$ \\
Blood glucose (mM) & $6 \pm 1$ & $32 \pm 1^{*}$ & $31 \pm 1^{*}$ \\
\hline Glyoxal ( $\mu \mathrm{M})$ & $18.5 \pm 0.4$ & $19.7 \pm 0.9$ & $18.2 \pm 0.3^{\#}$ \\
Methylglyoxal ( $\mu \mathrm{M})$ & $6.3 \pm 1.3$ & $24.7 \pm 11^{*}$ & $16.8 \pm 3.7^{*}$ \\
3-Deoxyglucasone (nM) & $196 \pm 5$ & $619 \pm 48^{* * *}$ & $629 \pm 20^{* * *}$ \\
\hline CML (pmol/mg protein) & $4.8 \pm 0.5$ & $10.5 \pm 1.0^{* * *}$ & $10.6 \pm 1.1^{* * *}$ \\
CEL (pmol/mg protein) & $7.4 \pm 0.7$ & $15.1 \pm 1.3^{* * *}$ & $13.6 \pm 1.3^{* * *}$ \\
MG-H1 (pmol/mg protein ) & $211 \pm 10$ & $245 \pm 10^{*}$ & $201 \pm 9^{\# \#}$ \\
\hline MDA ( $\mu$ mol/mg creatinine) & $0.02 \pm 0.004$ & $2.9 \pm 0.2^{* * *}$ & $3.8 \pm 0.2^{* * * \# \#}$ \\
Heart rate (bpm) & $340 \pm 12$ & $261 \pm 6^{* * *}$ & $284 \pm 9^{* * *}$ \\
Mean arterial pressure (mmHg) & $91 \pm 2$ & $59 \pm 3^{* * *}$ & $76 \pm 3^{* * * \# \#}$ \\
\hline
\end{tabular}

" $p<0.05$ compared with control, ${ }^{* * *} p<0.001$ compared with control. ${ }^{\#} p<0.05$ compared with diabetic, ${ }^{\# \#} p<$ 0.01 compared with diabetic, ${ }^{\# \#} \mathrm{p}<0.001$ compared with diabetic.

\section{Cardiac effects of diabetes and glyoxalase-I overexpression}

After 24 weeks of hyperglycaemia the hearts of the diabetic animals were hypertrophic (indicated by the increased organ to bodyweight ratio) as compared with the control animals. GLO-I overexpression reduced this, although not statically significant (Table 2a). Overall, cardiac weight and the thickness and volume of the left ventricle cardiac wall were significantly decreased in the diabetic animals and could not be significantly improved by GLO-I overexpression (Table 2a). Morphometric analyses of LV tissue sections of the heart showed that the percentage of collagen was increased in diabetic rats and could be decreased by GLO-I overexpression. Nevertheless, cardiomyocyte cross-sectional surface area did not significantly differ between the three groups (Table 2a). 
Table 6.2a. Effect of STZ and GLO-I overexpression on cardiac morphology.

\begin{tabular}{lccc}
\hline & Control & Diabetic & Diabetic GLO-I \\
\hline Cardiac weight $(\mathrm{g})$ & $1.32 \pm 0.08$ & $1.080 \pm 0.05^{*}$ & $0.993 \pm 0.04^{* *}$ \\
Heart to bodyweight $(\%)$ & $0.276 \pm 0.01$ & $0.360 \pm 0.01^{* * *}$ & $0.333 \pm 0.01^{* *}$ \\
\hline Thickness ventricle wall & & & \\
during diastole $(\mathrm{cm})$ & $0.17 \pm 0.01$ & $0.14 \pm 0.01^{* *}$ & $0.15 \pm 0.01^{*}$ \\
during systole $(\mathrm{cm})$ & $0.19 \pm 0.01$ & $0.17 \pm 0.01^{* *}$ & $0.17 \pm 0.01^{*}$ \\
\hline Volume ventricle wall & & & $0.45 \pm 0.02$ \\
during diastole $\left(\mathrm{cm}^{3}\right)$ & $0.49 \pm 0.03$ & $0.40 \pm 0.03^{*}$ & $0.40 \pm 0.02$ \\
during systole $\left(\mathrm{cm}^{3}\right)$ & $0.43 \pm 0.03$ & $0.39 \pm 0.02$ & $4.14 \pm 0.6$ \\
Fibrosis & & & $260 \pm 11$ \\
Collagen $(\%)$ & $3.41 \pm 0.3$ & $4.67 \pm 0.6^{*}$ & $284 \pm 21$ \\
Cardiomyocyte CSA $\left(\mu \mathrm{m}^{3}\right)$ & $277 \pm 12$ & $\mathrm{p}<0.001 \mathrm{compared}^{*}$ with control.
\end{tabular}

To further characterize the diabetic heart, we analyzed mRNA expression levels of several genes involved in hypertrophy, fibrosis, and inflammation. On mRNA level, we measured an increase in the hypertrophic markers ANP and BNP, which could not be restored by the GLO-I intervention (Table 2b). Markers for cardiac fibrosis Col1a1 and MMP-2 were decreased in the diabetic rats and were normalized by GLO-I overexpression. Also MMP-9, which was elevated in the diabetic heart, could be normalized by GLO-I overexpression. However, the fibrotic marker CTGF and the inflammation markers PAI-1 and TNF $\alpha$ were increased in the diabetic animals, but this was not attenuated by GLO-I overexpression.

Table 6.2b. Effect of STZ and GLO-I overexpression on cardiac gene expression.

\begin{tabular}{|c|c|c|c|}
\hline & Control & Diabetic & Diabetic GLO-I \\
\hline \multicolumn{4}{|l|}{ Hypertrophy } \\
\hline ANP ( $\%$ from control) & $100 \pm 15$ & $499 \pm 139^{* *}$ & $385 \pm 48^{* * *}$ \\
\hline BNP & $100 \pm 16$ & $148 \pm 23^{*}$ & $157 \pm 13^{* *}$ \\
\hline \multicolumn{4}{|l|}{ Fibrosis } \\
\hline CTGF & $100 \pm 13$ & $180 \pm 15^{* * *}$ & $197 \pm 23^{* *}$ \\
\hline Col1a1 & $100 \pm 13$ & $65 \pm 7^{*}$ & $109 \pm 14^{\#}$ \\
\hline MMP-2 & $100 \pm 6$ & $78 \pm 6^{*}$ & $115 \pm 10^{\# \#}$ \\
\hline MMP-9 & $100 \pm 23$ & $149 \pm 35$ & $128 \pm 22$ \\
\hline \multicolumn{4}{|l|}{ Inflammation } \\
\hline PAI-1 & $100 \pm 13$ & $278 \pm 28^{* * *}$ & $360 \pm 44^{* * *}$ \\
\hline TNF $\alpha$ & $100 \pm 14$ & $167 \pm 23^{* * *}$ & $193 \pm 16^{* * *}$ \\
\hline
\end{tabular}

$* \mathrm{p}<0.05$ compared with control, $* * \mathrm{p}<0.01$ compared with control, $* * * p<0.001$ compared with control. \# $\mathrm{p}<$ 0.05 compared with diabetic, \#\# $p<0.01$ compared with diabetic.

Cardiac function of the animals was also monitored by ultrasound recordings of the heart under isoflurane anaesthesia. There were no significant differences in end diastolic volume, end systolic volume, stroke volume, fractional shortening and ejection fraction between the three groups. Nevertheless, diabetic animals showed a significant decrease in cardiac output, logically caused by the observed decrease in heart rate. Intervention with 
GLO-I overexpression did not improve this hyperglycaemia-induced decrease in cardiac output (see Table $2 c$ ).

Table 6.2c. Effect of STZ and GLO-I overexpression on cardiac function.

\begin{tabular}{lccc}
\hline & Control & Diabetic & Diabetic GLO-I \\
\hline End diastolic volume $(\mathrm{mL})$ & $0.534 \pm 0.04$ & $0.536 \pm 0.04$ & $0.492 \pm 0.02$ \\
End systolic volume $(\mathrm{mL})$ & $0.211 \pm 0.02$ & $0.218 \pm 0.01$ & $0.205 \pm 0.01$ \\
Stroke volume $(\mathrm{mL})$ & $0.322 \pm 0.03$ & $0.318 \pm 0.03$ & $0.287 \pm 0.02$ \\
Fractional shortening (\%) & $16.8 \pm 1.0$ & $16.0 \pm 1.2$ & $16.6 \pm 0.8$ \\
Ejection fraction (\%) & $59.4 \pm 3.1$ & $58.9 \pm 1.2$ & $58.0 \pm 2.2$ \\
CO (mL/min) & $110.5 \pm 9.8$ & $81.2 \pm 7.2^{*}$ & $77.8 \pm 6.5^{*}$ \\
\hline
\end{tabular}

*p $<0.05$ compared with control.

\section{Vascular effects of diabetes and glyoxalase-I overexpression}

The results so far suggests an improvement in vascular function, i.e. greater peripheral resistance, rather than cardiac function by GLO-I overexpression, thereby preventing the haemodynamic fall in blood pressure. To investigate this further, we measured the contractile function of two vascular beds involved in blood pressure regulation, i.e. the renal artery and mesenteric resistance arteries. In the mesenteric arteries the maximal contractions to a combination of high potassium $(125 \mathrm{mM})$ and phenylephrine $(10 \mu \mathrm{M})$ did not differ between the control and diabetic groups (maximum contraction for $C, D$ and DG were; $3.58 \pm 0.26,3.68 \pm 0.33$ and $3.31 \pm 0.25 \mathrm{~N} / \mathrm{m}$ respectively). Also the dose-response curve to increasing amounts of phenylephrine did not differ between groups (see figure $2 A)$. However, the dose-response curve to potassium was significantly shifted in diabetic rats $(p<0.05)$ and was normalized by the GLO-I overexpression $(p<0.05)$ (see figure $2 B$ ).

In the renal arteries, the maximal contraction also did not differ between the groups (maximum contraction for C, D and DG were; $5.65 \pm 0.70,5.67 \pm 0.76$ and $5.60 \pm 0.42 \mathrm{~N} / \mathrm{m}$ respectively). Although there was no difference in contraction between control and diabetic rats, GLO-I overexpression resulted in an enhancement of contraction by both phenylephrine $(p<0.05$; figure $2 C$ ) and potassium ( $p<0.05$; figure $2 D)$. 


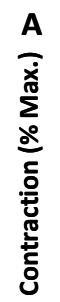

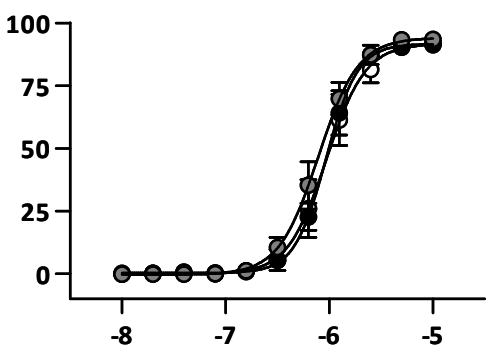

Log PHE (M)

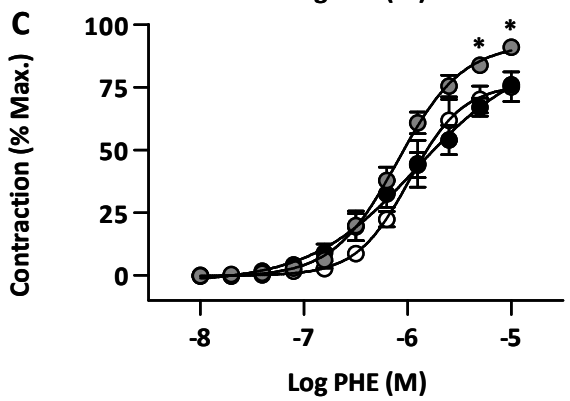

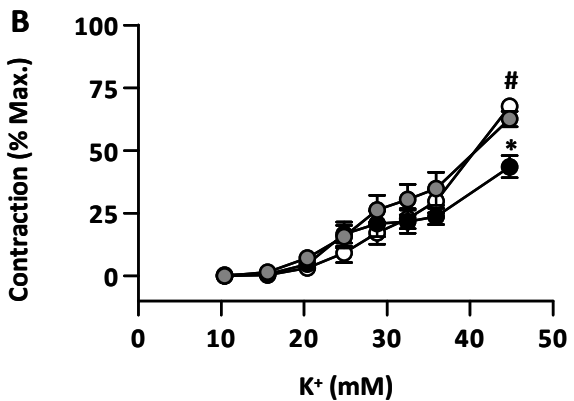

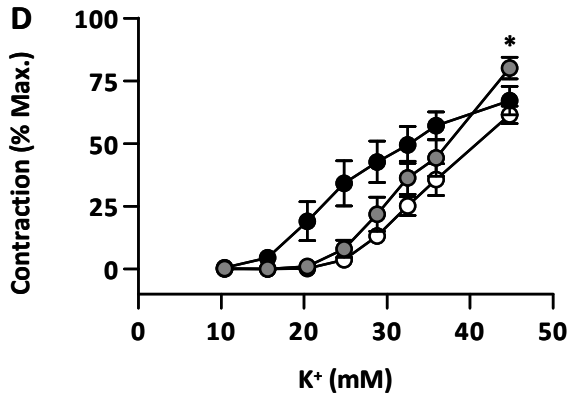

Figure 6.2. Effect of diabetes and GLO-I overexpression on vascular contraction in mesenteric and renal arteries. The white circles are the control rats, the black circles the wild-type diabetics and the grey circles are the diabetic rats with a GLO-I overexpression. A. Phenylephrine (PHE)-induced vasoconstriction after 24 weeks of diabetes in mesenteric arteries of control, wild-type diabetic, and GLO-I diabetic rats. B. Potassium-induced vasoconstriction after 24 weeks of diabetes in mesenteric arteries of control, wild-type diabetic, and GLO-I diabetic rats. C. PHE-induced vasoconstriction after 24 weeks of diabetes in renal arteries of control, wild-type diabetic, and GLO-I diabetic rats. D. Potassium-induced vasoconstriction after 24 weeks of diabetes in renal arteries of control, wild-type diabetic, and GLO-I diabetic rats.

\section{Discussion}

In this study we found that overexpression of GLO-I partially prevents the observed hyperglycaemia-induced mean arterial blood pressure depression in diabetic rats, without affecting cardiac function. The enhanced vascular contractile function of the diabetic GLOI overexpressing rat suggests a vascular rather than a cardiac compensation to prevent haemodynamic failing.

We previously reported that GLO-I overexpression in diabetic rats results in a decrease of oxo-aldehydes, AGEs and oxidative stress after 12 weeks of diabetes (14). We also reported that mesenteric arterial tissue levels of the major AGEs MG-H1 and CML are increased after 12 and 24 weeks of diabetes and that this can be prevented by GLO-I overexpression (Brouwers et al. 2011, submitted). In the present study we show that also after 24 weeks of diabetes GLO-I overexpression (partially) prevents (M)GO accumulation measured in blood and thereby normalizes circulating MG-H1 levels. However the 
decrease in circulating AGEs by the GLO-I overexpression was less effective after 24 weeks than after 12 weeks of STZ, indicated by the mild effect on CML and CEL. An overall increase of ROS during this prolonged exposure to hyperglycaemia and a subsequent decrease in the in vivo bioavailability and activity of both reduced glutathione and GLO-I could be the reasons for this observation.

Furthermore our current study shows that, despite some alterations in gene expression and morphology of the heart, 24 weeks of diabetes did not affect cardiac function, regarding to the amount of stroke volume produced by the heart. Also the fractional shortening and ejection fraction were not altered by the STZ injection. Nevertheless, the heart rate of the diabetic rats was decreased, which resulted in a decreased calculated cardiac output, without any effect of the GLO-I overexpression. Data about the effect of STZ on cardiac function and especially diabetes induced left ventricle dysfunction are conflicting. Some studies reported about early systolic LV dysfunction of the left ventricle in STZ-induced diabetic cardiomyopathy $(17 ; 18)$, while other studies $(19 ; 20)$, including our own data, showed no differences in LV function between diabetic and control rats. Also a study of Hoit et al. did not see any basal contractile dysfunction in STZ rats (21). Differences in strain, timeframe, or detection method can contribute to the divergent observations (22).

Diabetes-associated changes in cardiovascular function in the STZ-induced rat model are not entirely consistent with alterations observed in diabetic patients. STZ rats are characterized by a depression in heart rate and mean arterial blood pressure compared with control rats (23-25), which was also observed in our study. In contrast to the animal model, some clinical studies indicate that type I diabetes is associated with hypertension (26; 27) while others show no significant change in blood pressure $(28 ; 29)$. Reasons for this discrepancy could be that hypertension in humans is typically associated with longterm disease and that these individuals often have atherosclerotic changes and impaired vascular compliance. Observed differences between the STZ-induced rat model of diabetes and the clinical situation may also result from species variation and the origin of diabetes (single chemical insult versus naturally occurring). The animal model also differs from the clinical situation in that the hyperglycaemia in diabetic rats is not treated, and observed changes in cardiovascular and autonomic nervous function occur in the presence of continually elevated blood glucose, which is not the case in humans.

In STZ rats, the exact mechanisms underlying the heart rate and mean arterial blood pressure depression are not fully understood. Besides cardiac changes, also vascular dysfunction can contribute to the decrease in blood pressure in the STZ rat. This can be caused by a decrease in vascular contractile function, an increase in vasorelaxation or both. This is partially in agreement with our current results, which show that the vascular contraction in diabetic rats is impaired and that GLO-I overexpression prevents this in 
mesenteric arteries. In renal arteries there is no difference between wild-type diabetic and control rats, however vascular contraction is enhanced in the GLO-I transgenic rats.

Several studies conducted in aortas illustrate methodological explanations for this decreased vascular contractions and blood pressure in diabetes. A study by Rebolledo et al. demonstrated that the observed decrease in blood pressure was accompanied by alterations in the vascular response to noradrenalin in isolated rat aorta (30). The diabetic aorta exhibit a marked decrease of ${ }^{45}$ Calcium uptake, resulting in a depressed contraction in the slow, but not the fast, response to noradrenalin. The same group showed in a later study that also the relaxing function of the aorta is impaired in STZ rats (31). His study showed an accelerated relaxation of aortic smooth muscle when suddenly exposed to a $\mathrm{Ca}^{2+}$-free environment, which was associated with hyperactivity of sarco/endoplasmic reticulum $\mathrm{Ca}^{2+}$-ATPase. In respect to the involvement of MGO in this pathogenesis, MGO incubations of aorta segments resulted in impaired vascular contraction and increased endothelium-independent relaxation, both mediated via opening of smooth muscle BKca channels $(32 ; 33)$.

Furthermore increased relaxation and decreased contraction can also be the result of a increase in iNOS activity resulting in more NO production, and consequently ONOOformation. This nitrosative stress is also a mechanism of both heart and blood pressure depression $(34 ; 35)$.

In addition to the changes in vascular reactivity, there is at least one other mechanisms which can contribute to the depressed blood pressure in STZ rats. STZ-induced diabetes results in a depressed function of the autonomic nervous system already 24 hours after injection (36). In our diabetic GLO-I overexpressing model we did not directly address the function of the autonomic nervous function. Nevertheless unpublished data of our group in these animals show that diabetes results in sensory neuropathy, indicated by an increased latency during a hot plate assay. Interestingly GLO-I overexpression prevented this in the same magnitude as the blood pressure fall (approximately 50\%), indicating a role of AGEs in the development of diabetic neuropathy. Indeed, a recent study of DuranJimenez et al. showed that glycation of endoneurial extracellular matrix proteins impairs neurite outgrowth, thereby providing a mechanism for the failure of collateral sprouting and axonal regeneration in diabetic neuropathy (37). Another study by Bierhaus et al. shows that also prevention of the activation of the receptor for AGE (RAGE) prevents diabetes induced loss of pain perception in diabetic RAGE knock-out animals (38).

In summary, this study shows that overexpression of GLO-I detoxifies (methyl)glyoxal and thereby decreases (M)GO-derived AGEs in diabetic rats. The fact that GLO-I overexpression is associated with higher blood pressures and improved vascular function ex vivo, but not cardiac function, suggests that GLO-I protects arterial rather than cardiac function after STZ induced diabetes in rats. 


\section{Acknowledgements}

The authors gratefully acknowledge Jacques Debets (Department of Pharmacology and Toxicology, Maastricht University, Maastricht, the Netherlands), Theo Roemen (Department of Physiology, Maastricht University, Maastricht, the Netherlands), Marjo van den Waarenburg (Department of Internal Medicine, Maastricht University, Maastricht, the Netherlands), Jean Scheijen (Department of Internal Medicine, Maastricht University, Maastricht, the Netherlands), and Rob Barto (Department of Clinical Chemistry, VU University Medical Centre, Amsterdam, the Netherlands), for their excellent technical assistance. 


\section{References}

1. The effect of intensive treatment of diabetes on the development and progression of long-term complications in insulin-dependent diabetes mellitus. The Diabetes Control and Complications Trial Research Group. N Engl J Med 329:977-986, 1993

2. Brownlee M: Biochemistry and molecular cell biology of diabetic complications. Nature 414:813-820, 2001

3. Thornalley PJ: Glyoxalase l--structure, function and a critical role in the enzymatic defence against glycation. Biochem Soc Trans 31:1343-1348, 2003

4. Fosmark DS, Torjesen PA, Kilhovd BK, Berg TJ, Sandvik L, Hanssen KF, Agardh CD, Agardh E: Increased serum levels of the specific advanced glycation end product methylglyoxal-derived N $\delta$-(5-hydro-5-methyl-4-imidazolon2-yl)-L-ornithine are associated with retinopathy in patients with type 2 diabetes mellitus. Metabolism 55:232236, 2006

5. Ogawa S, Nakayama K, Nakayama M, Mori T, Matsushima M, Okamura M, Senda M, Nako K, Miyata T, Ito S: Methylglyoxal is a predictor in type 2 diabetic patients of intima-media thickening and elevation of blood pressure. Hypertension 56:471-476

6. Fosmark DS, Berg JP, Jensen AB, Sandvik L, Agardh E, Agardh CD, Hanssen KF: Increased retinopathy occurrence in type 1 diabetes patients with increased serum levels of the advanced glycation endproduct $\mathrm{N} \delta$-(5hydro-5-methyl-4-imidazolon-2-yl)-L-ornithine. Acta Ophthalmol 87:498-500, 2009

7. Beisswenger PJ, Drummond KS, Nelson RG, Howell SK, Szwergold BS, Mauer M: Susceptibility to diabetic nephropathy is related to dicarbonyl and oxidative stress. Diabetes 54:3274-3281, 2005

8. Bugger H, Abel ED: Rodent models of diabetic cardiomyopathy. Dis Model Mech 2:454-466, 2009

9. Norton GR, Candy G, Woodiwiss AJ: Aminoguanidine prevents the decreased myocardial compliance produced by streptozotocin-induced diabetes mellitus in rats. Circulation 93:1905-1912, 1996

10. Chang KC, Hsu KL, Tseng CD, Lin YD, Cho YL, Tseng YZ: Aminoguanidine prevents arterial stiffening and cardiac hypertrophy in streptozotocin-induced diabetes in rats. Br J Pharmacol 147:944-950, 2006

11. Wu MS, Liang JT, Lin YD, Wu ET, Tseng YZ, Chang KC: Aminoguanidine prevents the impairment of cardiac pumping mechanics in rats with streptozotocin and nicotinamide-induced type 2 diabetes. $\mathrm{Br} \mathrm{J} \mathrm{Pharmacol}$ 154:758-764, 2008

12. Corbett JA, Tilton RG, Chang K, Hasan KS, Ido Y, Wang JL, Sweetland MA, Lancaster JR, Jr., Williamson JR, McDaniel ML: Aminoguanidine, a novel inhibitor of nitric oxide formation, prevents diabetic vascular dysfunction. Diabetes 41:552-556, 1992

13. Giardino I, Fard AK, Hatchell DL, Brownlee M: Aminoguanidine inhibits reactive oxygen species formation, lipid peroxidation, and oxidant-induced apoptosis. Diabetes 47:1114-1120, 1998

14. Brouwers O, Niessen PM, Ferreira I, Miyata T, Scheffer PG, Teerlink T, Schrauwen P, Brownlee M, Stehouwer CD, Schalkwijk CG: Overexpression of glyoxalase-I reduces hyperglycaemia-induced levels of advanced glycation endproducts and oxidative stress in diabetic rats. J Biol Chem, 2010

15. Scheijen JL, van de Waarenburg MP, Stehouwer CD, Schalkwijk CG: Measurement of pentosidine in human plasma protein by a single-column high-performance liquid chromatography method with fluorescence detection. J Chromatogr B Analyt Technol Biomed Life Sci 877:610-614, 2009

16. Halpern W, Mulvany MJ: Tension responses to small length changes of vascular smooth muscle cells [proceedings]. J Physiol 265:21P-23P, 1977

17. Akula A, Kota MK, Gopisetty SG, Chitrapu RV, Kalagara M, Kalagara S, Veeravalli KK, Gomedhikam JP: Biochemical, histological and echocardiographic changes during experimental cardiomyopathy in STZ-induced diabetic rats. Pharmacol Res 48:429-435, 2003

18. Mihm MJ, Seifert JL, Coyle CM, Bauer JA: Diabetes related cardiomyopathy time dependent echocardiographic evaluation in an experimental rat model. Life Sci 69:527-542, 2001

19. Irlbeck M, Zimmer HG: Functional responses of the left and right heart of diabetic rats to alpha- and betaadrenergic receptor stimulation. Diabetes Res Clin Pract 31 Suppl:S79-86, 1996

20. Schenk J, Hebden RA, Dai S, McNeill JH: Integrated cardiovascular function in the conscious streptozotocindiabetic deoxycorticosterone-acetate-hypertensive rats. Pharmacology 48:211-215, 1994

21. Hoit BD, Castro C, Bultron G, Knight S, Matlib MA: Noninvasive evaluation of cardiac dysfunction by echocardiography in streptozotocin-induced diabetic rats. J Card Fail 5:324-333, 1999

22. Rodrigues B, Cam MC, Kong J, Goyal RK, McNeill JH: Strain differences in susceptibility to streptozotocininduced diabetes: effects on hypertriglyceridemia and cardiomyopathy. Cardiovasc Res 34:199-205, 1997 
23. Cheng X, Cheng XS, Kuo KH, Pang CC: Inhibition of iNOS augments cardiovascular action of noradrenaline in streptozotocin-induced diabetes. Cardiovasc Res 64:298-307, 2004

24. Szabo C, Zingarelli B, Salzman AL: Role of poly-ADP ribosyltransferase activation in the vascular contractile and energetic failure elicited by exogenous and endogenous nitric oxide and peroxynitrite. Circ Res 78:10511063, 1996

25. Bidani AK, Picken M, Hacioglu R, Williamson G, Griffin KA: Spontaneously reduced blood pressure load in the rat streptozotocin-induced diabetes model: potential pathogenetic relevance. Am J Physiol Renal Physiol 292:F647-654, 2007

26. Ambepityia G, Kopelman PG, Ingram D, Swash M, Mills PG, Timmis AD: Exertional myocardial ischemia in diabetes: a quantitative analysis of anginal perceptual threshold and the influence of autonomic function. J Am Coll Cardiol 15:72-77, 1990

27. Sundkvist G, Lilja B, Almer LO: Abnormal diastolic blood pressure and heart rate reactions to tilting in diabetes mellitus. Diabetologia 19:433-438, 1980

28. Mildenberger RR, Bar-Shlomo B, Druck MN, Jablonsky G, Morch JE, Hilton JD, Kenshole AB, Forbath N, McLaughlin PR: Clinically unrecognized ventricular dysfunction in young diabetic patients. J Am Coll Cardiol 4:234-238, 1984

29. Paillole C, Dahan M, Paycha F, Solal AC, Passa P, Gourgon R: Prevalence and significance of left ventricular filling abnormalities determined by Doppler echocardiography in young type I (insulin-dependent) diabetic patients. Am J Cardiol 64:1010-1016, 1989

30. Rebolledo A, Ayala-Paredes F, Milesi V, Grassi AO, Rinaldi GJ: Short-term streptozotocin-induced diabetes induces blood pressure decrease associated with reduced aortic $(45) \mathrm{Ca}(2+)$ uptake and selective depression of the sustained noradrenergic contraction. Diabetes Metab 27:40-48, 2001

31. Rinaldi GJ: Blood pressure fall and increased relaxation of aortic smooth muscle in diabetic rats. Diabetes Metab 31:487-495, 2005

32. Mukohda M, Yamawaki H, Nomura H, Okada M, Hara Y: Methylglyoxal inhibits smooth muscle contraction in isolated blood vessels. J Pharmacol Sci 109:305-310, 2009

33. Mukohda M, Yamawaki H, Okada M, Hara Y: Methylglyoxal enhances sodium nitroprusside-induced relaxation in rat aorta. J Pharmacol Sci 112:176-183

34. Takakura K, Xiaohong W, Takeuchi K, Yasuda Y, Fukuda S: Deactivation of norepinephrine by peroxynitrite as a new pathogenesis in the hypotension of septic shock. Anesthesiology 98:928-934, 2003

35. Nagareddy PR, Xia Z, MacLeod KM, McNeill JH: N-acetylcysteine prevents nitrosative stress-associated depression of blood pressure and heart rate in streptozotocin diabetic rats. J Cardiovasc Pharmacol 47:513-520, 2006

36. Monckton G, Pehowich E: Autonomic neuropathy in the streptozotocin diabetic rat. Can J Neurol Sci 7:135142,1980

37. Duran-Jimenez B, Dobler D, Moffatt S, Rabbani N, Streuli CH, Thornalley PJ, Tomlinson DR, Gardiner NJ: Advanced glycation end products in extracellular matrix proteins contribute to the failure of sensory nerve regeneration in diabetes. Diabetes 58:2893-2903, 2009

38. Bierhaus A, Haslbeck KM, Humpert PM, Liliensiek B, Dehmer T, Morcos M, Sayed AA, Andrassy M, Schiekofer S, Schneider JG, Schulz JB, Heuss D, Neundorfer B, Dierl S, Huber J, Tritschler H, Schmidt AM, Schwaninger M, Haering HU, Schleicher E, Kasper M, Stern DM, Arnold B, Nawroth PP: Loss of pain perception in diabetes is dependent on a receptor of the immunoglobulin superfamily. J Clin Invest 114:1741-1751, 2004 
General discussion

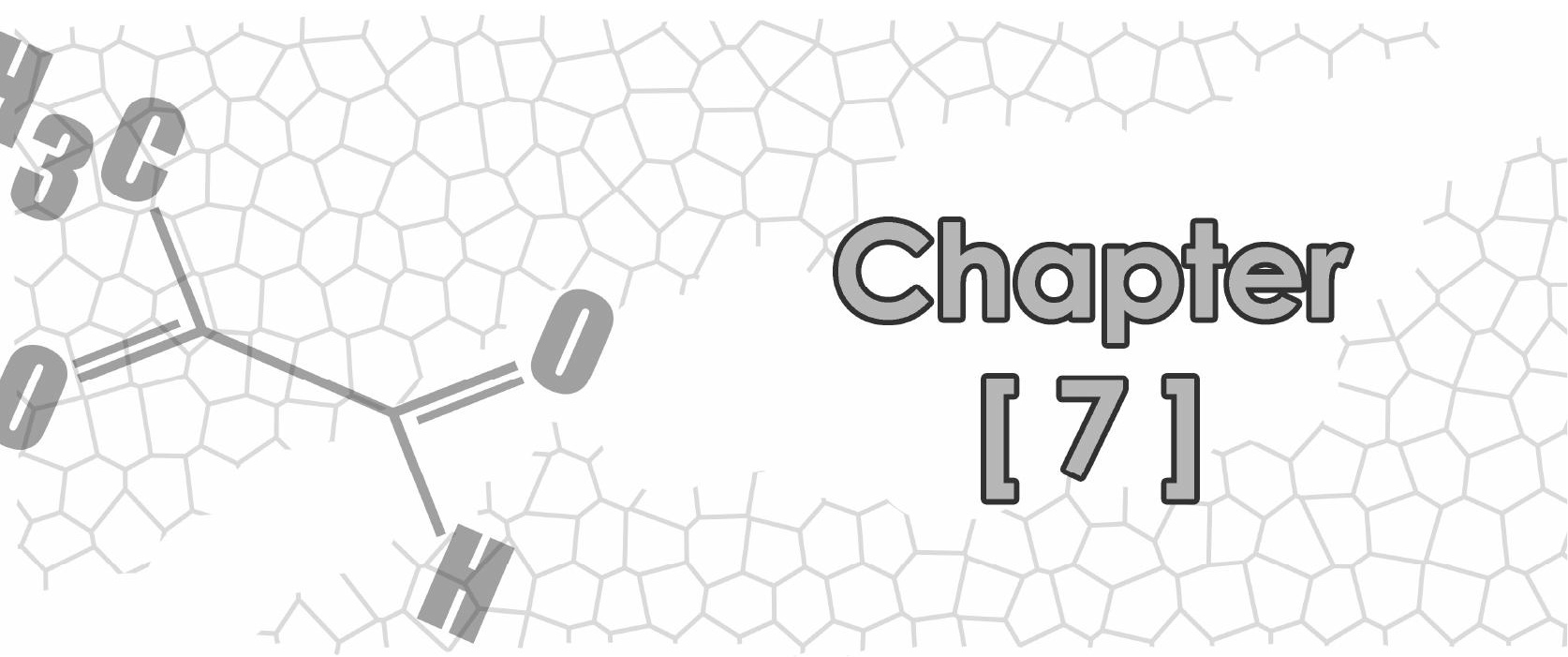




\section{Summary}

Vascular complications are responsible for most of the excess morbidity and mortality observed in diabetic patients. The primary causal factor in the majority of diabetic vascular complications is the prolonged exposure to hyperglycaemia and its detrimental effects on endothelial cell function. In this thesis we tested the hypothesis that intracellular formation of advanced glycation endproducts (AGEs) leads to endothelial dysfunction and consequently vascular complications. The highly reactive dicarbonyl compound methylglyoxal (MGO) is believed to be the major source of intracellular AGEs, and therefore this thesis mainly focuses on this pathway. Our studies provide important new insights in the effects of MGO and MGO-derived AGEs on endothelial cell function at the cellular (Chapter 2 and 3), but also at the vascular level (Chapter 3, 4 and 5). By making use of a novel glyoxalase-I (GLO-I) overexpressing model in rats we were also able to investigate the contribution of the GLO-I sensitive oxo-aldehyde MGO, but also of glyoxal (GO), and of (M)GO-derived AGEs, to the development of in vivo diabetic complications like nephropathy and altered haemodynamic function (Chapter 5 and 6). The current chapter will further summarize and discuss the findings of our studies. 


\section{MGO and endothelial cell damage in vitro}

To understand the involvement of MGO and MGO-derived AGEs in hyperglycaemiainduced vascular damage, several studies have focused on cellular pathways by direct stimulation of cultured endothelial or other vascular cells. The overall results of these studies show that MGO has cytotoxic properties, which are exerted due to oxidative stress $(1 ; 2)$, genotoxicity $(3 ; 4)$ and apoptosis $(5-7)$. The appearance of these effects in time seems to be consecutive, but somewhat overlapping.

However, one point of concern in these studies is the high concentration of MGO which is used. The in vivo concentration of MGO is under debate, and the relevance of the dose in cell studies therefore remains unclear. In general, MGO levels increase two- to four-fold in diabetic patients compared with controls (8-11). However, it is interesting to note that the increase in MGO levels in diabetic patients in the study of Beisswenger et al. (0.19 $\mu \mathrm{M})(8)$ is far below the control levels observed in the studies by Nemet et al. (0.65 $\mu \mathrm{M})(10)$ and Lapolla et al. (0.52 $\mu \mathrm{M})$ (9). In Chapter 4 we report blood MGO levels in non-diabetic rats of $0.66 \mu \mathrm{M}$ which increase to $0.87 \mu \mathrm{M}$ after 12 weeks of diabetes.

It should also be emphasized that extracellular and intracellular levels of MGO could be rather different. A study by Randell et al. showed that tissue MGO levels in rats were an order of a magnitude higher compared with their plasma levels (12). In cultured Chinese hamster ovary cells, Chaplen et al. reported that intracellular levels of MGO can be even up to $300 \mu \mathrm{M}$, which is considerably higher than the levels observed in blood samples (13). So it should be noticed that there is no consensus on the physiological concentration range of MGO yet and it is likely that the different methods used in different studies yield different results. With regard to selecting the appropriate concentration of MGO for in vitro incubations, two studies showed that only a fraction of the extracellularly added MGO reaches the cytoplasmatic environment. Experiments in a rat myoblast cell line with labelled MGO pointed out that only 3.1.\% of the added MGO reaches the cytoplasmatic region (14), while in aortic smooth muscle cells a comparable amount of $1.8 \%$ has been reported (15).

The effect of MGO on endothelial cells specifically is largely unknown. In Chapter $\mathbf{3}$ we demonstrated that incubation of endothelial cells with MGO in the concentrations 100, 330 and $1000 \mu \mathrm{M}$ for 60 minutes resulted in an increase of oxidative stress. Theoretically these concentrations may correspond with approximately 2.5, 10 and $25 \mu \mathrm{M}$ cytosolic $\mathrm{MGO}$, and this range is likely to be comparable with (patho)physiological concentrations in vivo. Under these conditions we measured a concentration-dependent increase of the fluorescent product of the probe $\mathrm{H}_{2}$ DCFDA. This probe is highly specific for the detection of oxidative stress, as evidenced by its inhibition by the antioxidant $\mathrm{N}$-acetylcysteine (NAC), but does not discriminate between the specific reactive oxygen species (ROS) which are involved. However, the observation that superoxide dismutase analogues EUK-134 
and $\mathrm{Mn}$ (III)TMP led to the reduction of MGO-induced oxidative stress, indicates that the formation of superoxide is involved. The results of our study were recently confirmed by Miyazawa et al. who focused on the involvement of the specific ROS and its origin in more detail (16). Their study showed that MGO incubation results predominantly in mitochondrial-derived superoxide production which further reacts to peroxynitrite and hydrogen-peroxide. They also demonstrate that the major cytosolic ROS source NADPH oxidase does not play a role in MGO-induced oxidative stress. However, elucidating the exact ROS source remains difficult due to the lack of specificity of most inhibitors. For example, the NADPH oxidase inhibitors DPI and apocynin also inhibit nitric oxide synthase (NOS) or have antioxidant properties respectively $(17 ; 18)$.

Despite the increase in MGO-induced oxidative stress, under the conditions which we used in our study, as described in Chapter 3, we could not detect any signs of decreased cell viability. The cell viability assay (MTT assay) showed no significant levels of cytotoxicity after 60 minutes of incubation with 100, 330 and $1000 \mu \mathrm{M}$ MGO. Therefore, we can conclude that the observed short-term effects with the used concentrations and cell type are independent of cell-cytotoxicity. In Chapter $\mathbf{3}$ we also describe that direct incubation of the $\mathrm{H}_{2}$ DCFDA probe with MGO (in a cell free setting) does not lead to any increase in fluorescent DCF signal, thereby excluding a non-specific MGO effect on the probe. This is in agreement with a study of Nakayama et al., who showed that MGO itself is not a free radical or ROS (19).

\section{Effects of MGO and MGO-derived AGEs on the eNOS pathway in vitro}

One of the possible effects of MGO or MGO-induced ROS in endothelial cells could be the interaction with nitric oxide (NO) production and/or bioavailability. In Chapter $\mathbf{3}$ we demonstrate that extracellularly added MGO results in intracellular accumulation of the MGO-derived AGE $\quad \mathrm{N}_{\delta}$-(5-hydro-5-methyl-4-imidazolon-2-yl)-L-ornithine (MG-H1). To investigate the possible direct effects of intracellular MGO and MGO-derived AGEs on eNOS activity and NO we performed two sets of non-cell culture based in vitro experiments. In Chapter $\mathbf{2}$ we showed that MGO, and the MGO-derived AGEs, MG-H1 and arginine-pyrimidine (AP), despite their high similarity with the endogenous eNOS inhibitor asymmetric dimethylarginine (ADMA), do not directly inhibit eNOS activity in whole cell homogenates of human endothelial cells. This lack of inhibition was not due to imperfections of the system, because in the same set of experiments ADMA, but not its inactive analogue symmetric dimethylarginine (SDMA), inhibited eNOS activity in the same manner as described earlier (20). However, Dhar et al. showed that MGO decreased eNOS activity by inhibiting phosphorylation on serine 1177 , a process which is also known to be regulated by ROS (21), without affecting total eNOS protein levels (22). The lack of effect of MGO in our study is probably due to the use of whole cell homogenates instead of 
intact cultured cells, which provide different experimental conditions. According to these results we can conclude that MGO has an inhibiting effect on eNOS activity, but this is not due to a direct effect.

The accumulation of AGEs in cells or the extracellular matrix can also result in increased quenching of NO (23). In Chapter 3 we showed in an in vitro setting that free MGO, but not highly modified MGO-albumin, also leads to increased breakdown of NO. The results show that MGO reduced the half-life of NO in buffer from $4.3 \pm 0.1$ minutes to approximately $0.8 \pm 0.2$ minutes, which corresponds with a rate constant of $1.1 \mathrm{M}^{-1} \mathrm{~s}^{-1}$. Under the same experimental conditions the rate constant found for the potent NO quenching molecule haemoglobin was $1.0 \times 10^{4} \mathrm{M}^{-1} \mathrm{~s}^{-1}$, thereby questioning the in vivo importance of NO quenching by MGO. The fact that Bucala et al. did find an effect of AGEs on NO could be due to the variation in the preparation of the AGE-modified proteins (23). The adducts they used were prepared by incubating albumin with glucose or glucose-6phosphate for 8 weeks, a method which is known to generate an increase in a wide variety of AGEs. Our adducts were specifically MGO-derived and characterisation of the modified albumin showed predominantly $\mathrm{N}_{\varepsilon}$-(1-carboxyethyl)lysine (CEL) and MG-H1 modifications.

\section{Receptor-mediated damage by MGO-modified proteins}

Increased formation of intracellular MGO can also theoretically contribute to the pool of circulating AGEs, which can subsequently activate cells via a RAGE-dependent pathway. Some research indeed shows that interaction of MGO-modified albumin with monocytes or macrophages results in activation and subsequent synthesis and secretion of tumour necrosis factor alpha (TNF- $\alpha$ ), interleukin-1 $\beta$ and monocyte colony stimulating factor (2426). However these studies did not investigate via which receptor this process was initiated. In addition, control experiments including blockage of the receptor involved, which could be found in later research concerning AGE/RAGE activation, were also absent. A study by Lieuw-a-Fa et al. showed that incubation of endothelial cells with MGOmodified albumin did not result in activation of these cells (27). They suggest that this is due to limitations of the in vitro single cell culture or a mutation in the endothelial RAGE receptor. In addition, it cannot be excluded that the previously observed effects of MGOderived adducts are biased due to bacterial contamination of the adducts. Therefore the puzzle whether MGO-derived AGEs can initiate signal transduction pathways via a receptor-mediated pathway remains to be solved. 


\section{Acute effects of MGO and MGO-derived AGEs on vascular function ex vivo}

Research in vitro has delivered us an enormous amount of data concerning the basic understanding of which pathways can be involved in hyperglycaemia-induced endothelial dysfunction. To actually address the function of the endothelium and its interplay with other cellular systems, like the smooth muscle cells, research in more complex systems is needed. To study the effect of MGO and MGO-derived AGEs on vascular function we used isolated mesenteric arteries. After precontraction, these arteries can be triggered with endothelium-dependent vasoactive compounds like acetylcholine (ACh) or endotheliumindependent compounds like the NO-donor sodium-nitroprusside (SNP). In the mesenteric arteries three pathways of ACh-induced relaxation are active: prostacyclin-, NO- and EDHF-dependent relaxation. In this thesis the eNOS/NO pathway was our main focus, and we addressed the effect of both ex vivo high glucose or MGO incubations and in vivo hyperglycaemia in rat mesenteric arteries.

In Chapter $\mathbf{3}$ we describe the effect of MGO incubations on mesenteric arteries. Under the same experimental conditions as we have used in the endothelial cell culture experiments, we measured a decrease in ACh-induced NO-mediated vascular relaxation. In agreement with the endothelial cell culture experiments, we also found an MGO-induced increase in oxidative stress, as indicated by elevated nitrotyrosine residues, in the mesenteric vascular wall. Prevention of this oxidative stress by preincubation with NAC, EUK-134, Mn(III)TMP and apocynin all normalised the impaired vascular reactivity. Additional experiments with a high glucose exposure of mesenteric arteries with GLO-I overexpressing confirmed the ROS-dependent impairment of NO-mediated relaxation by elevated intracellular MGO levels. Due to the very short exposure time of the arteries to elevated levels of MGO, glucose and ROS, alterations in the protein levels of eNOS can be excluded as an explanation for this impaired relaxation. Therefore, a decreased NO bioavailability by the interaction of NO with superoxide is a likely explanation for the observed impaired reactivity. However, a limitation of this study was that we did not investigate the effect of MGO/ROS on eNOS phosphorylation, and therefore it cannot be excluded that the impaired endothelial function was caused by decreased eNOS activity.

A study by Xu et al. showed that CML can impair vascular reactivity in rabbit aortic rings, by a RAGE-dependent pathway (28). However there is currently no evidence that MGOderived AGEs can activate the endothelial RAGE receptor and thereby lead to altered cell function (27). Because this lack of evidence could be due to limitations of the in vitro cell culture setting, we incubated mesenteric arteries ex vivo with MGO-modified albumin. We could not find any alterations in vascular reactivity under any of the conditions and modifications tested. Nevertheless, the observation that the RAGE ligand S100b did 
significantly impair vascular reactivity indicates that the system is active under our experimental settings.

In conclusion these data show that hyperglycaemia-induced impaired vascular reactivity is mediated by intracellular MGO levels in a pathway dependent on oxidative stress.

\section{Chronic effects of MGO and MGO-derived AGEs on vascular function ex vivo}

To investigate the chronic in vivo effects of MGO we first measured vascular reactivity in mesenteric arteries of rats after 12 and 24 weeks of streptozotocin (STZ)-induced diabetes. Our experiments in Chapter $\mathbf{5}$ show that diabetes impairs endotheliumdependent relaxation, which is in accordance with many other studies $(29 ; 30)$. We also used mesenteric arteries of diabetic rats with a GLO-I overexpression and showed that these rats have improved endothelial function compared with wild-type diabetic rats. After 24 weeks of diabetes endothelial dysfunction was more pronounced than after 12 weeks of diabetes, however the beneficial effect of GLO-I overexpression was comparable. Despite that other studies showed decreased protein and mRNA levels of eNOS after STZinduced diabetes, we could not confirm this in our study $(31 ; 32)$. Therefore it is reasonable to speculate that long-term hyperglycaemia results in decreased NO bioavailability by a MGO/ROS-dependent pathway. This is also supported by data of other groups which show increased ROS production and decreased endothelium-dependent relaxation in diabetes $(23 ; 29 ; 33 ; 34)$. However, an indirect effect of MGO on eNOS activity cannot be excluded. It should also be emphasized that phosphorylation of eNOS by Akt is an important eNOS regulating mechanism in response to insulin, vascular endothelial growth factor, and other ligands. However, because the signalling mechanism involved in ACh-stimulated NO production is mostly calcium-dependent (35) it is not likely that decreased eNOS phosphorylation is the cause of the observed impaired reactivity. This should be addressed in further studies.

\section{Effect of GLO-I overexpression on the levels of MGO and MGO-derived AGEs in vivo}

Microangiopathy is a direct result of chronic hyperglycaemia and the main course of major clinical microvascular complications, such as nephropathy, cardiomyopathy, retinopathy and neuropathy. In this pathogenesis, dysfunction of the endothelium is an important factor in both the initiation and progression of vascular complications. We showed that MGO contributes to the development of endothelial dysfunction and to investigate whether this leads to vascular complications in diabetes, we used the STZ-induced diabetes model in rats. 
These rats develop a clinical syndrome characterised by hyperglycaemia, which is similar to human diabetes. Moreover, STZ-diabetic rats develop the same chronic microvascular complications as observed in diabetic patients (36).

Phillips et al. characterised the glyoxalase system in several tissues of the STZ-induced diabetic rat (37). They observed that diabetes decreased GLO-I activity in the kidney and sciatic nerve, but GLO-I activity was increased in the lens, skeletal muscle and red blood cells. Nevertheless in all tissues, diabetes increased MGO levels, indicating that the minimally compensatory increase in GLO-I activity was not sufficient to decrease MGO levels. The same was also observed by Staniszewska et al. in diabetic mouse lenses (38). We used the STZ model to study the concept that transgenic GLO-I overexpression could possibly lead to decreased levels of AGEs in transgenic diabetic rats compared with wildtype diabetic rats. Indeed we found that after 12 weeks of diabetes (Chapter 4) GLO-I overexpression decreased circulating levels of GO and MGO by approximately $80 \%$ compared with wild-type animals, without affecting 3-DG levels. Furthermore GO- and MGO-derived AGEs were all decreased by $50 \%$ which also resulted in a equivalent decrease of oxidative stress markers. Despite comparable observations in vitro (39) and in lower organisms in vivo (40), our study was the first to describe that in vivo GLO-I overexpression in rats is a good model to investigate $\mathrm{GO}$ and MGO-derived AGE detoxification. In addition we also performed experiments in a prolonged model of diabetes. We therefore sacrificed the rats after 24 weeks of diabetes as described in Chapter $\mathbf{5}$ and $\mathbf{6}$. After 24 weeks, levels of GO,MGO and MG-H1 were again decreased by GLO-I overexpression, however the effect on CML and CEL was less than after 12 weeks of diabetes. A limitation in our study is that we did not measure levels of both reduced (GSH) and oxidized glutathione (GSSG). Depletion of GSH, and an increase in GSSG, could be a reason for the diminished effect of GLO-I overexpression after 24 weeks of diabetes compared with 12 weeks. If long-term diabetes indeed hampers the beneficial effect of GLO-I overexpression, supplementation with GSH could be an option to prevent this in future experiments.

\section{Effects of MGO on renal and cardiovascular function in vivo}

In Chapter $\mathbf{5}$ we showed that endothelial dysfunction and AGE levels are closely related to the development of early renal impairment. In our STZ model, early urinary markers of diabetic nephropathy (albumin, lipocalin, osteopontin and kidney injury molecule-1) were increased in wild-type diabetic rats and could be attenuated after 24 weeks by GLO-I overexpression. Until now the AGE-inhibitors aminoguanidine and pyridoxamine were the only tools used to investigate the role of intracellular glycation in diabetic nephropathy. Although both compounds lead to good results in animal models of vascular complications, aminoguanidine is known to be toxic for humans $(41 ; 42)$. 
Phase 2 trials for pyridoxamine demonstrate that it is well tolerated in patients and that it has a positive impact on serum creatinine and urinary TGF- $\beta$ levels (43).

However, aminoguanidine and pyridoxamine are not specific for MGO and therefore the observed effects cannot be directly attributed to MGO or MGO-derived AGEs. Our study therefore is the first study showing the important role of intracellular glycation by MGO in the development of early diabetic nephropathy. Nevertheless to address the involvement of MGO in more advanced diabetic nephropathy perhaps another model should be used. A study of Bidani et al. speculated that blood pressure depression in STZ rats is probably one of the reasons for the only modest nephrotic damage observed in the STZ diabetes model (44). Indeed we also observed this decrease in blood pressure in parallel with the mildly nephrotic phenotype in our study. Therefore diabetic models with increased blood pressure, like for example prolonged STZ combined with hypertension, are probably better models to investigate advanced diabetic nephropathy.

In diabetic patients there exists an increased risk for the development of heart failure, independent of coronary artery disease and hypertension. Although this diabetic cardiomyopathy is increasingly recognized, the underlying mechanisms are still incompletely understood. Nevertheless, research in animal models, and especially the STZ-induced diabetic model, resulted in conflicting results. One of the most important reasons for this is that functional alterations in heart function are not consistently found in the STZ model. Also our study, described in Chapter 6, did not observe any impairment of cardiac function in the diabetic animals regarding stroke volume, ejection fraction and fractional shortening. However we could observe differences in gene expression markers of fibrosis and also in fibrotic tissue in the left ventricle of the heart. These levels of fibrosis could be improved by GLO-I overexpression, indicating a role of intracellular glycation in this process. We also measured a decrease in intra-arterial blood pressure in the STZ-induced diabetic animals. The observation that GLO-I overexpression blunted the diabetes-induced fall in blood pressure independently of cardiac function suggests a vascular compensatory mechanism. Indeed, we observed enhanced contractions in the mesenteric and renal bed in the GLO-I compared with the wild-type diabetic rats. Despite that hypotension is not observed in humans with diabetes, these results show once again the beneficial effects of MGO detoxification on vascular function.

\section{Role of MGO in other vascular diseases}

Besides the beneficial effects of GLO-I overexpression on nephropathy and blood pressure regulation as we described in Chapter $\mathbf{5}$ and $\mathbf{6}$ of this thesis, we also collected preliminary data about the effects of GLO-I overexpression on the development of diabetic retinopathy and neuropathy. GLO-I overexpression was also shown to lower AGEs in the retina and thereby preventing the appearance of acellular capillaries and glial fibrillary 
acidic (GFAP) protein levels, both characteristics of diabetic retinopathy, after 12 and 24 weeks of diabetes (unpublished data in collaboration with Prof. Dr. A. Stitt, Queens University Belfast). Furthermore, recent observations in our own laboratory show that GLO-I activity in endothelial cells is decreased by hypoxia. Although the mechanism behind this is still elusive, it suggests that GLO-I also could be important in non-diabetic ischaemic diseases like myocardial infarctions, atherosclerosis and peripheral arterial disease. Indeed Kumagai et al. recently showed that GLO-I overexpression is useful in the prevention of renal ischaemia-reperfusion damage (45). Furthermore (M)GO detoxification by GLO-I could also play an important role in neuropathy, regarding our unpublished observation that GLO-I diabetic rats have an improved sensibility on a hot plate compared with wildtype diabetic rats. Indeed a recent study of Duran-Jimenez et al. shows that glycation impairs neurite outgrowth, thereby providing a mechanism for diabetic neuropathy (46).

\section{Alterations in the glyoxalase-I gene}

A study by Miyata et al. described a case report of a 69 years old lady which suffered from recurrent cardiovascular complications, despite the absence of significant risk factors. They observed very low levels and activities of GLO-I in this patient, which was associated with higher levels of (M)GO and their derived AGEs (47). Genetic analysis of the coding region of the GLO-I gene showed no alterations, and the cause of the reduced level of GLO-I remains therefore elusive. In general, the importance of single nucleotide polymorphisms (SNPS) in the GLO-I gene is still largely unknown. Some studies show that variability in the GLO-I gene is associated with cancer $(48 ; 49)$ and anxiety $(50)$. In addition a study of Junaid et al. identified a SNP in GLO-I as a risk factor for autism (51), while others could not confirm this association $(52 ; 53)$. With regard to cardiovascular diseases, SNPS in GLO-I were shown to be associated with cardiovascular and peripheral vascular disease in haemodialysis patients (54) and pro-thrombotic factors (55). The Ala111Glu change is the only SNP which directly changes the GLO-I structure and is commonly found to be associated with outcome, but also other SNPs could be involved. However our own group could not find any association of a range of SNPs, including Ala111Glu, with vascular complications in two different cohort studies of cardiovascular disease (56). If indeed alterations in the GLO-I gene only minimally contribute to the occurrence of cardiovascular complications, then this suggests that alterations on a functional level by post-transcriptional or even post-translational changes are probably more important (57). 


\section{Conclusion and further perspectives}

The knowledge about the involvement of dicarbonyls and AGEs in the development of diabetic complications has considerably expanded over the last years. Our findings in the novel STZ-induced diabetic GLO-I overexpressing rat have provided new insights concerning the contribution of the GLO-I sensitive oxo-aldehydes GO and MGO and their derived AGEs in the development of endothelial dysfunction and the progression to diabetic complications. Our studies show that MGO has detrimental effects on endothelial cell function, which is largely dependent on the formation of ROS. In vivo detoxification of MGO via GLO-I overexpression not only decreases the levels of MGO-derived AGEs, but also diminishes levels of oxidative stress in a rat model of diabetes. Furthermore, this detoxification improves endothelium-dependent relaxation, which is beneficial in preventing vascular complications like diabetic nephropathy. Furthermore GLO-I overexpression also prevents the observed diabetes-induced blood pressure depression in rats by increasing vascular resistance independently of cardiac function. The effects of MGO on the endothelial cell are summarized in figure 7.1.

The transgenic GLO-I overexpressing rat model pushes the glycation field forward. Nevertheless in future research we have to take it even one step further and try to measure vascular and organ function non-invasively in time. Our observations concerning vascular function in this model is largely restricted to cross-sectional data and mostly focuses on one organ or one vascular tree. With the current possibilities to use MRI, telemetry and contrast-enhanced ultrasound, integrating cardiovascular function longitudinally is within our possibilities and should be further addressed. Furthermore, another challenge should be to focus on MGO in other diseases. It is therefore noteworthy that the role of MGO emerges in non-hyperglycaemic diseases like renal ischaemia and hypertension $(45 ; 58)$.

Due to the development of novel animal models, excellent imaging techniques and reliable analytical tools the role of MGO and MGO-derived AGEs in cardiovascular diseases can be studied in more depth. Perhaps the most important additional challenge will be to elucidate how GLO-I activity is regulated. Knowledge about how to manipulate this important MGO-detoxifying enzyme will provide us with new therapeutic tools in the future. 


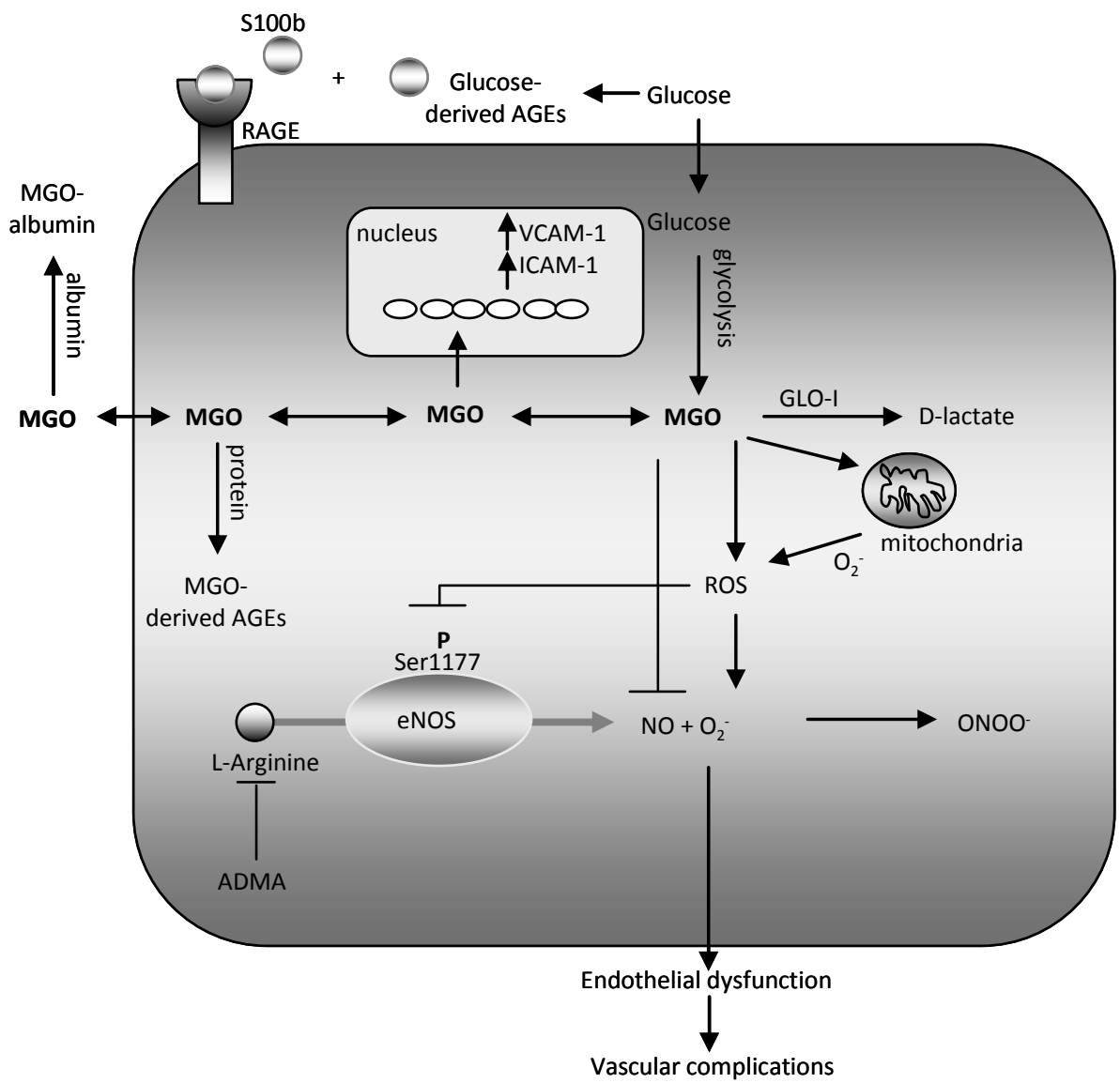

Figure 7.1. Mechanism of methylglyoxal-induced endothelial dysfunction

Diabetes-induced increased levels of MGO result in the accumulation of intracellular and extracellular MGOderived AGEs. These AGEs do not recognize the endothelial receptor for AGE (RAGE), and also do not directly inhibit the nitric oxide (NO) producing enzyme endothelial-NO-synthase (eNOS). However free MGO does lead to increased NO breakdown directly and via the production of superoxide (probably derived from the mitochondria) and consequently peroxynitrite. As a result these changes impair vascular reactivity in a pathway dependent on oxidative stress, thereby leading to systemic endothelial dysfunction and the development of vascular complications like nephropathy. These processes can be stopped by the detoxification of glyoxal (GO) and MGO by overexpression of the glyoxalase-I (GLO-I) enzyme. 


\section{References}

1. Vander Jagt DL, Hunsaker LA, Vander Jagt TJ, Gomez MS, Gonzales DM, Deck LM, Royer RE: Inactivation of glutathione reductase by 4-hydroxynonenal and other endogenous aldehydes. Biochem Pharmacol 53:11331140, 1997

2. $\mathrm{Wu} \mathrm{L}$, Juurlink $\mathrm{BH}$ : Increased methylglyoxal and oxidative stress in hypertensive rat vascular smooth muscle cells. Hypertension 39:809-814, 2002

3. Hou SM, Nori P, Fang JL, Vaca CE: Methylglyoxal induces hprt mutation and DNA adducts in human Tlymphocytes in vitro. Environ Mol Mutagen 26:286-291, 1995

4. Kang Y, Edwards LG, Thornalley PJ: Effect of methylglyoxal on human leukaemia 60 cell growth: modification of DNA G1 growth arrest and induction of apoptosis. Leuk Res 20:397-405, 1996

5. Chan WH, Wu HJ, Shiao NH: Apoptotic signaling in methylglyoxal-treated human osteoblasts involves oxidative stress, c-Jun N-terminal kinase, caspase-3, and p21-activated kinase 2. J Cell Biochem 100:1056-1069, 2007

6. Du J, Suzuki H, Nagase F, Akhand AA, Ma XY, Yokoyama T, Miyata T, Nakashima I: Superoxide-mediated early oxidation and activation of ASK1 are important for initiating methylglyoxal-induced apoptosis process. Free Radic Biol Med 31:469-478, 2001

7. Okado A, Kawasaki Y, Hasuike Y, Takahashi M, Teshima T, Fujii J, Taniguchi N: Induction of apoptotic cell death by methylglyoxal and 3-deoxyglucosone in macrophage-derived cell lines. Biochem Biophys Res Commun 225:219-224, 1996

8. Beisswenger PJ, Howell SK, Touchette AD, Lal S, Szwergold BS: Metformin reduces systemic methylglyoxal levels in type 2 diabetes. Diabetes 48:198-202, 1999

9. Lapolla A, Reitano R, Seraglia R, Sartore G, Ragazzi E, Traldi P: Evaluation of advanced glycation end products and carbonyl compounds in patients with different conditions of oxidative stress. Mol Nutr Food Res 49:685-690, 2005

10. Nemet I, Turk Z, Duvnjak L, Car N, Varga-Defterdarovic L: Humoral methylglyoxal level reflects glycemic fluctuation. Clin Biochem 38:379-383, 2005

11. Odani H, Shinzato T, Matsumoto Y, Usami J, Maeda K: Increase in three alpha,beta-dicarbonyl compound levels in human uremic plasma: specific in vivo determination of intermediates in advanced Maillard reaction. Biochem Biophys Res Commun 256:89-93, 1999

12. Randell EW, Vasdev S, Gill V: Measurement of methylglyoxal in rat tissues by electrospray ionization mass spectrometry and liquid chromatography. J Pharmacol Toxicol Methods 51:153-157, 2005

13. Chaplen FW, Fahl WE, Cameron DC: Evidence of high levels of methylglyoxal in cultured Chinese hamster ovary cells. Proc Natl Acad Sci U S A 95:5533-5538, 1998

14. Riboulet-Chavey A, Pierron A, Durand I, Murdaca J, Giudicelli J, Van Obberghen E: Methylglyoxal impairs the insulin signaling pathways independently of the formation of intracellular reactive oxygen species. Diabetes 55:1289-1299, 2006

15. Che W, Asahi M, Takahashi M, Kaneto H, Okado A, Higashiyama S, Taniguchi N: Selective induction of heparin-binding epidermal growth factor-like growth factor by methylglyoxal and 3-deoxyglucosone in rat aortic smooth muscle cells. The involvement of reactive oxygen species formation and a possible implication for atherogenesis in diabetes. J Biol Chem 272:18453-18459, 1997

16. Miyazawa N, Abe M, Souma T, Tanemoto M, Abe T, Nakayama M, Ito S: Methylglyoxal augments intracellular oxidative stress in human aortic endothelial cells. Free Radic Res 44:101-107

17. Heumuller S, Wind S, Barbosa-Sicard E, Schmidt HH, Busse R, Schroder K, Brandes RP: Apocynin is not an inhibitor of vascular NADPH oxidases but an antioxidant. Hypertension 51:211-217, 2008

18. Wang $\mathrm{YX}$, Poon $\mathrm{Cl}$, Poon KS, Pang CC: Inhibitory actions of diphenyleneiodonium on endothelium-dependent vasodilatations in vitro and in vivo. Br J Pharmacol 110:1232-1238, 1993

19. Nakayama M, Saito K, Sato E, Nakayama K, Terawaki H, Ito S, Kohno M: Radical generation by the nonenzymatic reaction of methylglyoxal and hydrogen peroxide. Redox Rep 12:125-133, 2007

20. Tsikas D, Boger RH, Sandmann J, Bode-Boger SM, Frolich JC: Endogenous nitric oxide synthase inhibitors are responsible for the L-arginine paradox. FEBS Lett 478:1-3, 2000

21. Hoshino S, Kikuchi Y, Nakajima M, Kimura H, Tsuyama S, Uemura K, Yoshida K: Endothelial NO Synthase (eNOS) phosphorylation regulates coronary diameter during ischemia-reperfusion in association with oxidative stress. Free Radic Res 39:481-489, 2005

22. Dhar A, Dhar I, Desai KM, Wu L: Methylglyoxal scavengers attenuate endothelial dysfunction induced by methylglyoxal and high concentrations of glucose. Br J Pharmacol 
23. Bucala R, Tracey KJ, Cerami A: Advanced glycosylation products quench nitric oxide and mediate defective endothelium-dependent vasodilatation in experimental diabetes. J Clin Invest 87:432-438, 1991

24. Abordo EA, Thornalley PJ: Synthesis and secretion of tumour necrosis factor-alpha by human monocytic THP1 cells and chemotaxis induced by human serum albumin derivatives modified with methylglyoxal and glucosederived advanced glycation endproducts. Immunol Lett 58:139-147, 1997

25. Westwood ME, Thornalley PJ: Induction of synthesis and secretion of interleukin 1 beta in the human monocytic THP-1 cells by human serum albumins modified with methylglyoxal and advanced glycation endproducts. Immunol Lett 50:17-21, 1996

26. Abordo EA, Westwood ME, Thornalley PJ: Synthesis and secretion of macrophage colony stimulating factor by mature human monocytes and human monocytic THP-1 cells induced by human serum albumin derivatives modified with methylglyoxal and glucose-derived advanced glycation endproducts. Immunol Lett 53:7-13, 1996

27. Lieuw-a-Fa ML, Schalkwijk CG, Engelse M, van Hinsbergh VW: Interaction of Nepsilon(carboxymethyl)lysineand methylglyoxal-modified albumin with endothelial cells and macrophages. Splice variants of RAGE may limit the responsiveness of human endothelial cells to AGEs. Thromb Haemost 95:320-328, 2006

28. Xu B, Chibber R, Ruggiero D, Kohner E, Ritter J, Ferro A: Impairment of vascular endothelial nitric oxide synthase activity by advanced glycation end products. FASEB J 17:1289-1291, 2003

29. Diederich D, Skopec J, Diederich A, Dai FX: Endothelial dysfunction in mesenteric resistance arteries of diabetic rats: role of free radicals. Am J Physiol 266:H1153-1161, 1994

30. Pflueger AC, Gross JM, Knox FG: Adenosine-induced renal vasoconstriction in diabetes mellitus rats: role of prostaglandins. Am J Physiol 277:R1410-1417, 1999

31. Hink U, Li H, Mollnau H, Oelze M, Matheis E, Hartmann M, Skatchkov M, Thaiss F, Stahl RA, Warnholtz A, Meinertz T, Griendling K, Harrison DG, Forstermann U, Munzel T: Mechanisms underlying endothelial dysfunction in diabetes mellitus. Circ Res 88:E14-22, 2001

32. Nagareddy PR, Xia Z, MacLeod KM, McNeill JH: N-acetylcysteine prevents nitrosative stress-associated depression of blood pressure and heart rate in streptozotocin diabetic rats. J Cardiovasc Pharmacol 47:513-520, 2006

33. Gao X, Zhang H, Schmidt AM, Zhang C: AGE/RAGE produces endothelial dysfunction in coronary arterioles in type 2 diabetic mice. Am J Physiol Heart Circ Physiol 295:H491-498, 2008

34. Su J, Lucchesi PA, Gonzalez-Villalobos RA, Palen DI, Rezk BM, Suzuki Y, Boulares HA, Matrougui K: Role of advanced glycation end products with oxidative stress in resistance artery dysfunction in type 2 diabetic mice. Arterioscler Thromb Vasc Biol 28:1432-1438, 2008

35. Fleming I, Busse R: Signal transduction of eNOS activation. Cardiovasc Res 43:532-541, 1999

36. Rees DA, Alcolado JC: Animal models of diabetes mellitus. Diabet Med 22:359-370, 2005

37. Phillips SA, Mirrlees D, Thornalley PJ: Modification of the glyoxalase system in streptozotocin-induced diabetic rats. Effect of the aldose reductase inhibitor Statil. Biochem Pharmacol 46:805-811, 1993

38. Staniszewska MM, Nagaraj RH: Upregulation of glyoxalase I fails to normalize methylglyoxal levels: a possible mechanism for biochemical changes in diabetic mouse lenses. Mol Cell Biochem 288:29-36, 2006

39. Shinohara M, Thornalley PJ, Giardino I, Beisswenger P, Thorpe SR, Onorato J, Brownlee M: Overexpression of glyoxalase-I in bovine endothelial cells inhibits intracellular advanced glycation endproduct formation and prevents hyperglycaemia-induced increases in macromolecular endocytosis. J Clin Invest 101:1142-1147, 1998

40. Schlotterer A, Kukudov G, Bozorgmehr F, Hutter H, Du X, Oikonomou D, Ibrahim Y, Pfisterer F, Rabbani N, Thornalley P, Sayed A, Fleming T, Humpert P, Schwenger V, Zeier M, Hamann A, Stern D, Brownlee M, Bierhaus A, Nawroth $\mathrm{P}$, Morcos M: C. elegans as model for the study of high glucose- mediated life span reduction. Diabetes 58:2450-2456, 2009

41. Bolton WK, Cattran DC, Williams ME, Adler SG, Appel GB, Cartwright K, Foiles PG, Freedman BI, Raskin P, Ratner RE, Spinowitz BS, Whittier FC, Wuerth JP: Randomized trial of an inhibitor of formation of advanced glycation end products in diabetic nephropathy. Am J Nephrol 24:32-40, 2004

42. Freedman BI, Wuerth JP, Cartwright K, Bain RP, Dippe S, Hershon K, Mooradian AD, Spinowitz BS: Design and baseline characteristics for the aminoguanidine Clinical Trial in Overt Type 2 Diabetic Nephropathy (ACTION II). Control Clin Trials 20:493-510, 1999

43. Williams ME, Bolton WK, Khalifah RG, Degenhardt TP, Schotzinger RJ, McGill JB: Effects of pyridoxamine in combined phase 2 studies of patients with type 1 and type 2 diabetes and overt nephropathy. Am J Nephrol 27:605-614, 2007 
44. Bidani AK, Picken M, Hacioglu R, Williamson G, Griffin KA: Spontaneously reduced blood pressure load in the rat streptozotocin-induced diabetes model: potential pathogenetic relevance. Am J Physiol Renal Physiol 292:F647-654, 2007

45. Kumagai T, Nangaku M, Kojima I, Nagai R, Ingelfinger JR, Miyata T, Fujita T, Inagi R: Glyoxalase I overexpression ameliorates renal ischemia-reperfusion injury in rats. Am J Physiol Renal Physiol 296:F912-921, 2009

46. Duran-Jimenez B, Dobler D, Moffatt S, Rabbani N, Streuli CH, Thornalley PJ, Tomlinson DR, Gardiner NJ: Advanced glycation end products in extracellular matrix proteins contribute to the failure of sensory nerve regeneration in diabetes. Diabetes 58:2893-2903, 2009

47. Miyata T, van Ypersele de Strihou C, Imasawa T, Yoshino A, Ueda Y, Ogura H, Kominami K, Onogi H, Inagi R, Nangaku M, Kurokawa K: Glyoxalase I deficiency is associated with an unusual level of advanced glycation end products in a hemodialysis patient. Kidney Int 60:2351-2359, 2001

48. Antognelli C, Del Buono C, Ludovini V, Gori S, Talesa VN, Crino L, Barberini F, Rulli A: CYP17, GSTP1, PON1 and GLO1 gene polymorphisms as risk factors for breast cancer: an Italian case-control study. BMC Cancer 9:115, 2009

49. Krechler T, Jachymova M, Mestek O, Zak A, Zima T, Kalousova M: Soluble receptor for advanced glycation end-products (sRAGE) and polymorphisms of RAGE and glyoxalase I genes in patients with pancreas cancer. Clin Biochem 43:882-886

50. Landgraf R, Kessler MS, Bunck M, Murgatroyd C, Spengler D, Zimbelmann M, Nussbaumer M, Czibere L, Turck CW, Singewald N, Rujescu D, Frank E: Candidate genes of anxiety-related behavior in HAB/LAB rats and mice: focus on vasopressin and glyoxalase-I. Neurosci Biobehav Rev 31:89-102, 2007

51. Junaid MA, Kowal D, Barua M, Pullarkat PS, Sklower Brooks S, Pullarkat RK: Proteomic studies identified a single nucleotide polymorphism in glyoxalase I as autism susceptibility factor. Am J Med Genet A 131:11-17, 2004 52. Rehnstrom K, Ylisaukko-Oja T, Vanhala R, von Wendt L, Peltonen L, Hovatta I: No association between common variants in glyoxalase 1 and autism spectrum disorders. Am J Med Genet B Neuropsychiatr Genet 147B:124-127, 2008

53. Wu YY, Chien WH, Huang YS, Gau SS, Chen CH: Lack of evidence to support the glyoxalase 1 gene (GLO1) as a risk gene of autism in Han Chinese patients from Taiwan. Prog Neuropsychopharmacol Biol Psychiatry 32:17401744,2008

54. Kalousova M, Jachymova M, Germanova A, Kubena AA, Tesar V, Zima T: Genetic predisposition to advanced glycation end products toxicity is related to prognosis of chronic hemodialysis patients. Kidney Blood Press Res 33:30-36

55. Gale CP, Futers TS, Summers LK: Common polymorphisms in the glyoxalase-1 gene and their association with pro-thrombotic factors. Diab Vasc Dis Res 1:34-39, 2004

56. Engelen L, Ferreira I, Brouwers O, Henry RM, Dekker JM, Nijpels G, Heine RJ, van Greevenbroek MM, van der Kallen CJ, Blaak EE, Feskens EJ, ten Cate H, Stehouwer CD, Schalkwijk CG: Polymorphisms in glyoxalase 1 gene are not associated with vascular complications: the Hoorn and CoDAM studies. J Hypertens 27:1399-1403, 2009

57. Birkenmeier G, Stegemann C, Hoffmann R, Gunther R, Huse K, Birkemeyer C: Posttranslational modification of human glyoxalase 1 indicates redox-dependent regulation. PLoS One 5:e10399

58. Ogawa S, Nakayama K, Nakayama M, Mori T, Matsushima M, Okamura M, Senda M, Nako K, Miyata T, Ito S: Methylglyoxal is a predictor in type 2 diabetic patients of intima-media thickening and elevation of blood pressure. Hypertension 56:471-476 
Chapter 7 


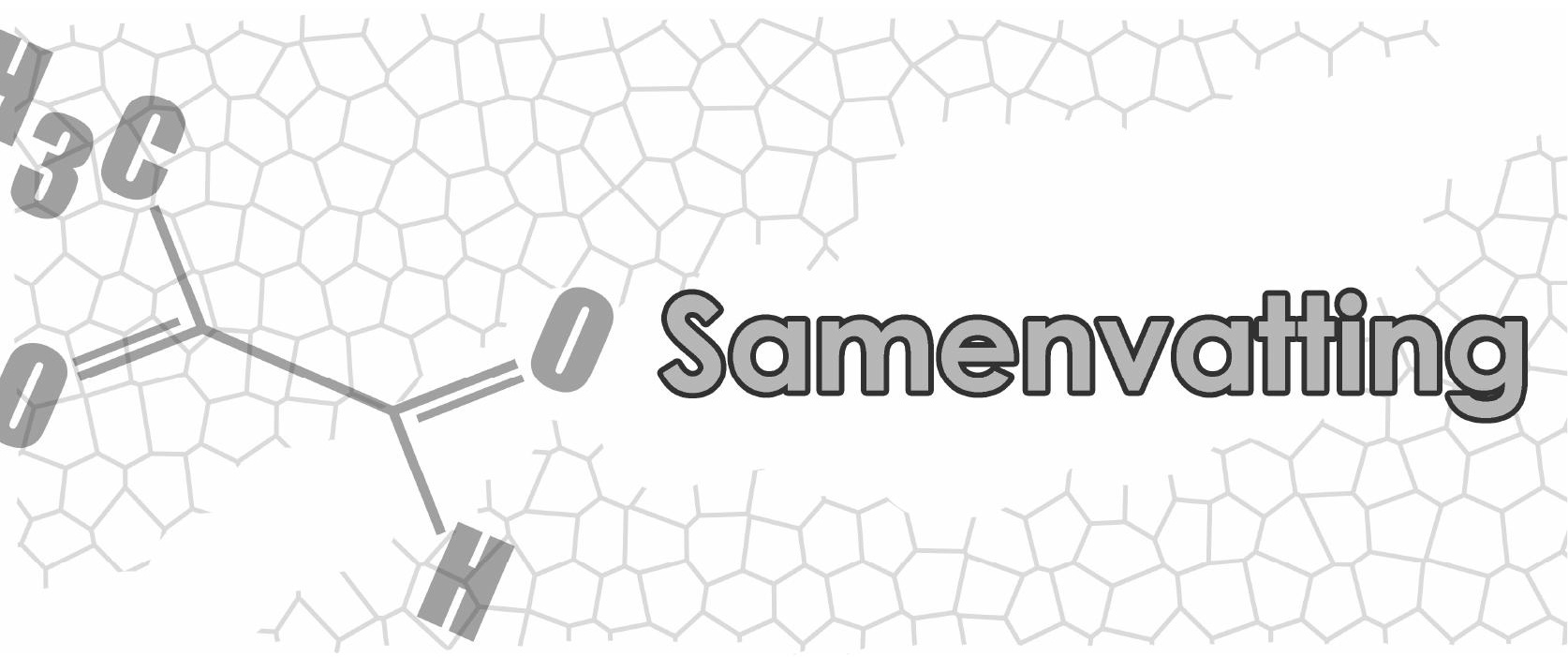


Diabetes mellitus is een metabole aandoening die gekarakteriseerd wordt door een chronische deficiëntie in de productie (type 1 diabetes; T1DM) of het effect van insuline (type 2 diabetes; T2DM). Dit defect resulteert in verhoogde bloedglucose spiegels, die vervolgens schade veroorzaken aan de bloedvaten en belangrijke sterk gevasculeerde organen, zoals de retina, de nieren, het hart en het zenuwstelsel. Langdurige blootstelling aan hyperglycemie is daardoor de belangrijkste causale factor in de ontwikkeling van diabetes gerelateerde complicaties.

Een belangrijk mechanisme waardoor hyperglycemie diabetische complicaties kan veroorzaken is de formatie van advanced glycation endproducts (AGEs). AGEs zijn een heterogene familie van niet-enzymatisch door koolhydraten gemodificeerde eiwitten, die verhoogd zijn in patiënten met diabetes. Er zijn meerdere mechanismen waardoor AGEs kunnen bijdragen aan het ontstaan van vasculaire complicaties. Deze zijn onder andere de interactie van glyceringsproducten met hun receptor, de formatie van cross-links in de basaal membraan en intracellulaire accumulatie van geglyceerde eiwitten.

Het reactieve oxoaldehyde methylglyoxaal (MGO), wat voornamelijk wordt gevormd als een bijproduct van de glycolyse, wordt gezien als de belangrijkste AGE "precursor" in de hyperglycemische endotheelcellen van de bloedvaten. De accumulatie van MGO wordt onder fysiologische omstandigheden voorkomen door het glyoxalase ontgiftingsmechanisme, waarin glyoxalase-I (GLO-I) het belangrijkste enzym is.

Er bestaat geen twijfel dat AGEs een rol spelen in de ontwikkeling van vasculaire complicaties in diabetes, maar de exacte modificaties en mechanismen die hierbij betrokken zijn, zijn nog grotendeels onbekend. Het belangrijkste doel van dit proefschrift was dan ook om de rol van intracellulair MGO en van MGO afkomstige AGEs in het ontstaan van endotheelschade en vasculaire complicaties te onderzoeken.

In Hoofdstuk 1, de algemene introductie van dit proefschrift, worden bovengenoemde onderwerpen, zoals hyperglycemische schade, de vorming en detoxificatie van MGO en de schadelijke effecten van glyceringsproducten uitgebreid besproken. Tevens wordt in dit hoofdstuk uiteengezet wat in dit proefschrift onderzocht is.

De mechanismen waardoor diabetes leidt to endotheeldisfunctie en vervolgens de ontwikkeling van orgaanschade zijn nog niet geheel opgehelderd. Een verstoring in de beschikbaarheid van het vaatverwijdende stikstof oxide (NO) lijkt echter een belangrijke stap te zijn in de ontwikkeling van vasculaire complicaties bij diabeten. Bij de synthese van $\mathrm{NO}$ is een oxidatie van het aminozuur L-arginine betrokken. L-Arginine analogen zoals het endogene asymmetrisch dimethylarginine (ADMA), staan bekend als remmers van het NO synthase enzym. Toename van intracellulair MGO in de endotheelcellen van diabeten leidt tot verhoogde spiegels van de arginine modificaties arginine-pyrimidine $(A P)$ en $\mathrm{N}_{\delta^{-}}(5-$ 
hydro-5-methyl-4-imidazolon-2-yl)-L-ornithine (MG-H1). Ondanks dat deze modificaties structurele homologie vertonen met bekende NO inhibitoren, laten we in Hoofdstuk 2 zien dat pathofysiologische waardes van vrij MGO, AP en MG-H1, in tegenstelling tot ADMA geen direct effect hebben op de activiteit van het endotheliale NO synthase enzym in vitro.

Een andere manier waarop de beschikbaarheid van NO verlaagd kan worden is door een versnelde afbraak. De vorming van oxidatieve stress door hyperglycemie en daarna de snelle reactie tussen vrije zuurstof radicalen met NO is een bekend fenomeen dat hiertoe bijdraagt. In Hoofdstuk $\mathbf{3}$ van dit proefschrift beschrijven we dat incubatie van endotheelcellen met MGO leidt tot een toename in MG-H1 en ook oxidatieve stress. Het feit dat we in aan MGO blootgestelde ex vivo geïsoleerde ratten arteriolen een toename van het oxidatieve NO product peroxynitriet detecteren, bevestigd de vermeende reactie tussen MGO geïnduceerde zuurstof radicalen (ROS) en NO. Ten gevolge van deze verlaagde beschikbaarheid van NO is de acetylcholine geïnduceerde endotheelafhankelijke vasculaire relaxatie beduidend lager in arteriolen die blootgesteld zijn aan MGO vergeleken met controle arteriolen. Experimenten in arteriolen afkomstig van ratten met een GLO-I overexpressie laten zien dat ook door hoog glucose geïnduceerde verslechtering van de vaatfunctie voor een groot deel afhankelijk is van MGO. Vermindering van oxidatieve stress door middel van antioxidanten normaliseert de vaatfunctie. Ondanks dat we ook aantonen dat MGO een klein direct effect heeft op de afbraaksnelheid van NO, lijkt de indirecte vorming van ROS de belangrijkste reden van de verslechterde vaatfunctie. De additionele observatie dat extracellulair MGO gemodificeerd albumine geen effect heeft op de vaatfunctie, versterkt de opvatting dat vooral intracellulaire verhoging van MGO belangrijke schadelijke effecten ten gevolge heeft.

Om de chronische effecten van hyperglycemie in vivo te bestuderen wordt er veelal gebruik gemaakt van ratten of muizen die diabeet gemaakt zijn met behulp van een streptozotocine (STZ) injectie. Om de rol van AGEs in de ontwikkeling van vasculaire complicaties te onderzoeken in dit proefdiermodel is er vóór het verschijnen van dit proefschrift vooral gebruik gemaakt van een interventie met de aspecifieke $\alpha$-oxoaldehyde "scavenger" aminoguanidine. In Hoofdstuk 4 beschrijven we een nieuw GLO-I overexpressie model in ratten met diabetes, waarmee het mogelijk werd om de betrokkenheid van MGO in de ontwikkeling van vasculaire complicaties nauwkeuriger te onderzoeken. Dit hoofdstuk laat zien dat GLO-I overexpressie in staat is om de door diabetes verhoogde spiegels van MGO, AGEs en oxidatieve stress te verlagen. De observatie dat essentiële eiwitten van de mitochondriële ademhalingsketen gereguleerd 
worden door MGO en GLO-I geeft weer dat mitochondriële ROS en glycering nauw aan elkaar verbonden zijn.

In Hoofdstuk 5 onderzochten we de potentiële positieve effecten van GLO-I overexpressie op vaat- en nierfunctie na 12 en 24 weken STZ geïnduceerde diabetes in ratten. Door gebruik te maken van geïsoleerde mesenteriale arteriolen ontdekten we dat de NO gemedieerde relaxatie verminderd was in de ratten met diabetes en dat dit verbeterd kon worden door GLO-I overexpressie. Ook de verhoogde expressie van markers voor endotheeldisfunctie zoals VCAM-1 en ICAM-1 kon worden voorkomen. Deze verbeterde vaatfunctie had verder tot gevolg dat de door diabetes veroorzaakte stijging in vroege markers van nefropathie afgezwakt kon worden. De sterke positieve correlatie tussen urinaire albumine, osteopontine, lipocaline- 2 en kidney injury molecule- 1 waardes en de plasma AGEs $\mathrm{N}_{\varepsilon}$-(1-carboxymethyl)lysine en $\mathrm{N}_{\varepsilon}$-(1-carboxyethyl)lysine, weerspiegelen de betrokkenheid van AGEs bij het ontstaan van vroege nierschade.

Ratten met STZ geïnduceerde diabetes worden gekenmerkt door hypotensie. Met behulp van hartechoscopie en intra-arteriele bloeddruk metingen ontdekten we dat ratten met diabetes en een GLO-I overexpressie, onafhankelijk van hun hartfunctie een hogere bloeddruk vertoonden dan de wildtype ratten met diabetes. Deze observatie staat beschreven in Hoofdstuk 6, en kan worden verklaard door een betere contractie van zowel mesenteriale als renale vaten in de GLO-I rat.

De resultaten van dit proefschrift worden in Hoofdstuk 7 samengevat en in relatie tot elkaar besproken. Er werd geconcludeerd dat MGO schadelijke effecten heeft op endotheelcel- en vaatfunctie, wat grotendeels afhankelijk is van de formatie van reactieve zuurstof radicalen. Verder laten we in een in vivo ratten model van diabetes zien dat detoxificatie van MGO door GLO-I overexpressie niet alleen de van MGO afkomstige AGEs verlaagd, maar ook oxidatieve stress gedeeltelijk voorkomt. Vervolgens verbetert deze detoxificatie ook de verminderde endotheelafhankelijke bloedvat relaxatie, de ontwikkeling van vroege nephropathie en diabetes geïnduceerde bloeddruk verlaging in ratten met type 1 diabetes. In de toekomst zal nieuwe kennis over de regulatie van het belangrijkste MGO detoxificerende enzym GLO-I, wellicht kunnen zorgen voor nieuwe aangrijpingspunten in de bestrijding van diabetes geïnduceerde vasculaire complicaties. 


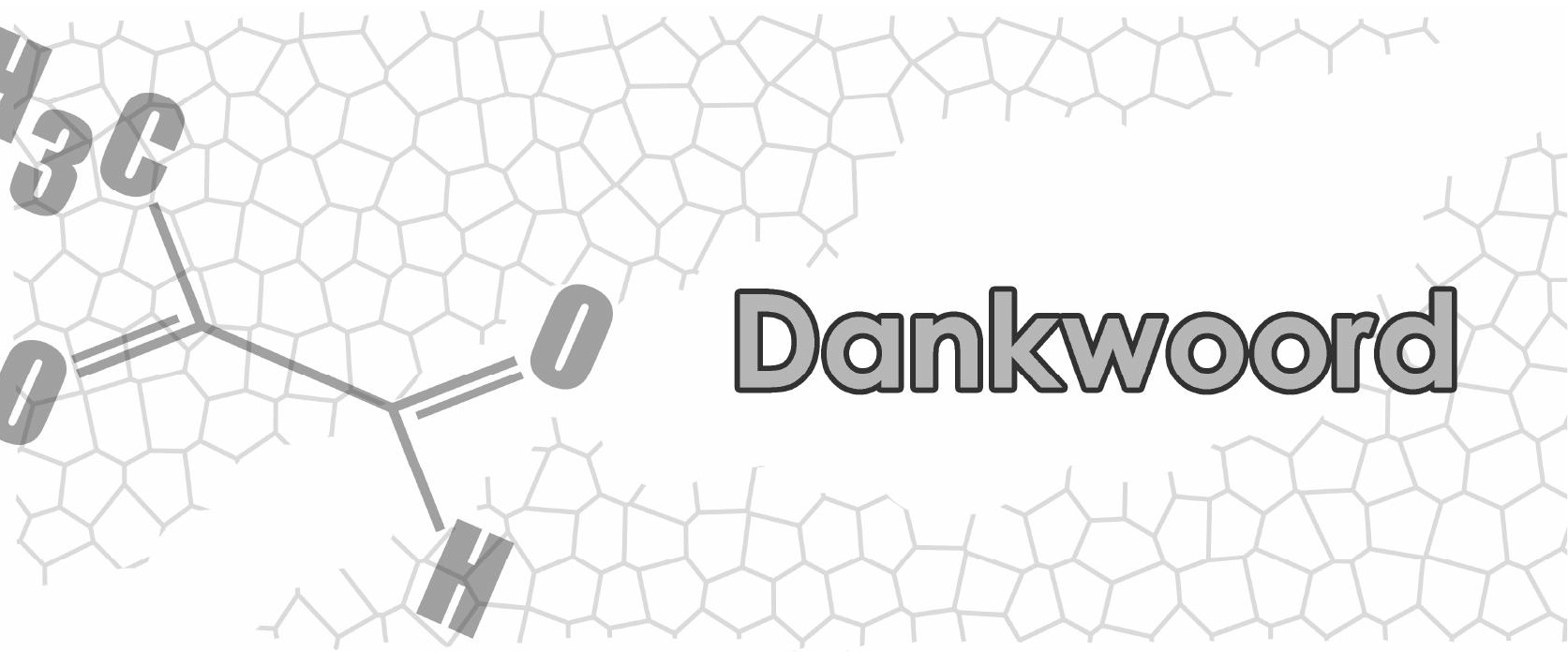


En dan nu... het meest gelezen stukje van dit proefschrift, en wellicht daarom ook één van de moeilijkste, het dankwoord! Dit stuk kan de reden zijn om je proefschrift toch maar "genaaid gebrocheerd" in plaats van "geplakt" te fabriceren (want voor dat andere populaire stukje, de losse stellingen, hoef je het boekje niet eens open te maken).

Naast een goed gelezen stuk, is het dankwoord natuurlijk ook een belangrijk onderdeel van het proefschrift. Onderzoek doe je namelijk niet alleen, en daarom deze parade van welgemeende bedankjes!

Als eerste Dr. C.G. Schalkwijk. Beste Casper, als ik maar één iemand zou mogen bedanken dan zou jij dat zeker zijn. Er is veel veranderd op de afdeling sinds ik in 2006 als eerste (mannelijke!) AIO in dienst trad, maar jouw betrokkenheid en heldere visie is nooit veranderd. Het aantal AIO's bleef zich maar uitbreiden, en daarmee ook de insteek in verschillende onderwerpen. Jij groeide echter mee, observeerde, begeleidde en corrigeerde, en dat allemaal op een hele prettige manier. De juiste mensen achter de knoppen en alle hoofden in de goede richting, dat heb je prima voor elkaar gekregen! Gelukkig is jouw richtingsgevoel in de wetenschap stukken beter dan dat in het dagelijkse leven. Of was dat een truc zodat jij je eens kon laten begeleiden door je eigen AIO('s)?

Ook wil ik graag mijn 2 promotores Prof. Dr. C.D.A. Stehouwer en Prof. Dr. J.G.R. De Mey bedanken. Beste Coen, bij goede begeleiding gaat het niet om de frequentie dat men elkaar spreekt, maar om de beschikbaarheid en de toewijding van de begeleider indien de te begeleiden AIO dit nodig heeft. We hebben niet vaak overleg (nodig) gehad, maar je was altijd $100 \%$ op de hoogte, duidelijk en zeer scherp (koppelingstekens!), dank hier voor! Beste Jo, je betrokkenheid bij mijn onderzoek en in het algemeen bij mijn begin in de academische wereld was groot. Als ik je zocht hoefde ik alleen het gezang op het lab te volgen! Onze gespreksonderwerpen varieerden van vasculaire functie tot reizen, maar waren altijd interessant, informeel en informatief.

I also would like to thank Prof. Dr. T. Miyata. Dear Toshio, without your pioneering vision and work constructing the GLO-I rodents, this work could not have been done. I hope that our current collaboration leads to (more) succesfull papers and will be continuated in the future. Furthermore, I would also like to thank all the co-authors of the accepted and submitted papers for their contribution, and the members of the assessment committee for reading this thesis.

Ik begon in augustus 2006, pfff wat gaat de tijd toch snel. Na een tijdje op het lab gezeten te hebben (daar waar een promovendus ook hoort... ;-)), mocht ik ruim 4 jaar geleden mijn intrede maken in kamertje 5.324. De brandjes van de verhitte discussies tussen Steven en Martijn waren net geblust toen ik de plaats naast Marjon kon innemen. Marjon, door alle 
proefpersonen zagen we elkaar meestal pas in de namiddag, maar we hadden een leuk tijd als kamergenootjes. ik hoop dat je nog even doorzet en nu de laatste loodjes kunt omzetten in goud.

Degene die het goud al binnen heeft wil ik zeker hartelijk bedanken voor al onze gesprekken over GLO-I, RAGE, AGEs, bruiloften, huizen en nog veel meer... Beste Katrien, nu weten we beide dat er in het leven minstens 3 dingen niet tot in detail te plannen zijn: promoveren, trouwen en bouwen! Maar ik zie jou als het goede voorbeeld en dan komt het seffens zeker en vast goe hè!

Verder wil ik ook kamergenoot Bas bedanken voor zijn (eigenwijze) discussies en interesse in alle biochemische mechanismes die eigenlijk ook achter de epidemiologie schuil gaan...

Natuurlijk wil ik ook de rest van de groep bedanken voor hun praktische en/of theoretische bijdrage en ook hun gezelligheid en interesse! En ach achteraf blijken zelfs computer Aio's ook gewoon mensen te zijn... ;-). Heel veel succes met promoveren, beurzen aanvragen, experimenteren en jullie verdere carrières.

Met name wil ik ook nog Jean en Marjo bedanken die van onschatbare waarde zijn geweest voor mijn onderzoek en voor onze hele afdeling!

In het bijzonder wil ik ook Petra bedanken. Het is dat ik geen kaas heb gegeten van sculpturen, anders maakte ik een standbeeld voor je. Daarom pak ik het iets kleiner aan, je krijgt een aparte alinea.... Wat is het toch een luxe, dat ik tijdens de leuke en minder leuke (coupes/vingers snijden... AUW!) onderdelen van het onderzoek zo een goede hulp heb gehad. Samenwerken met jou, een echte super "promotus analist", is echt synergie!

Naast mijn eigen afdeling heb ik ook vele (avond)uren doorgebracht in het farmacologie lab. Ik wil dan ook de mensen van die afdeling bedanken voor hun gastvrijheid en de interesse en bijdrage aan mijn onderzoek!

Ook wil ik alle medewerkers van het CPV, en met name Richard, Paulien en Rik bedanken voor hun belangrijke bijdrage aan de totstandkoming van dit boekje. Beste Richard, een veelgehoord antwoord op een vraag is: "Ik zoek het voor je op en dan laat ik het je weten." Jij onderscheid je echter van de rest door dit ook daadwerkelijk te doen! Hiervoor veel dank!

Buiten Maastricht ben ik ook nog een 4-tal maanden te gast geweest op de Fysiologie afdeling van het VUMC te Amsterdam. Helaas heeft het werk dat ik daar heb gedaan dit proefschrift niet gehaald, maar zou ik toch graag de hele afdeling en met name Ed, Wineke, en Caro-lynn willen bedanken voor een leerzame en gezellige tijd (borrelen en onderzoek gaan hand in hand!). 
Naast hun hulp, steun en collegialiteit wil ik ook graag een aantal mensen bedanken voor hun vriendschap. Amy, Matthijs, Rianne en Marcella met jullie heb ik een geweldig leuke tijd gehad. Dankzij de etentjes, drankjes, poppodium/bowl/pool/bioscoop bezoekjes (helaas zonder ridders), bewezen wij dat er meer is dan alleen werken! Maar eigenlijk zijn we nog veel te braaf geweest... we zullen in de toekomst er nog voor moeten zorgen dat de hele "bucket list" wordt afgewerkt! Jammer dat de afstanden langzaamaan steeds groter worden, maar gelukkig gaan Rianne en ik (logeerkamers genoeg straks) ervoor zorgen dat niet iedereen vanuit Limbabwe naar de Randstad trekt...

Verder wil ik ook graag mijn (schoon)familie bedanken. Pa en Ma, ik ben jullie ontzettend dankbaar voor de (studie) mogelijkheden die jullie mij in mijn leven geboden hebben. Claudia, mijn lieve zus, hartstikke bedankt voor je interesse en goede moed! Verder wil ik ook mijn "tentjes" bedanken voor hun steun; wat is het toch heerlijk om in het najaar even tot rust te komen in de Portugese zon!!! Ook mijn nieuwe ouders en broer, Jan, Anja en Bas (hoe is het met de ratjes?, goed!, ze slapen nu... ) wil ik ook heel graag bedanken voor hun interesse en steun.

Tot slot, mijn maatje, mijn vriendin en na dit jaar zelfs mijn vrouw, Corine! Ik ga niet zeggen dat het zonder jou niet gelukt was, want sommige dingen gaan zelfs sneller (maar niet altijd beter) als je niemand hebt om voor naar huis te gaan. Maar dit neemt niet weg dat de stelling "de beste onderzoekers zijn vrijgezel" het boekje terecht niet gehaald heeft. Door jouw positiviteit, zorg, steun en liefde weet ik nu één ding zeker: "de beste onderzoekers hebben juist een hele goede en fijne relatie".

Olaf 


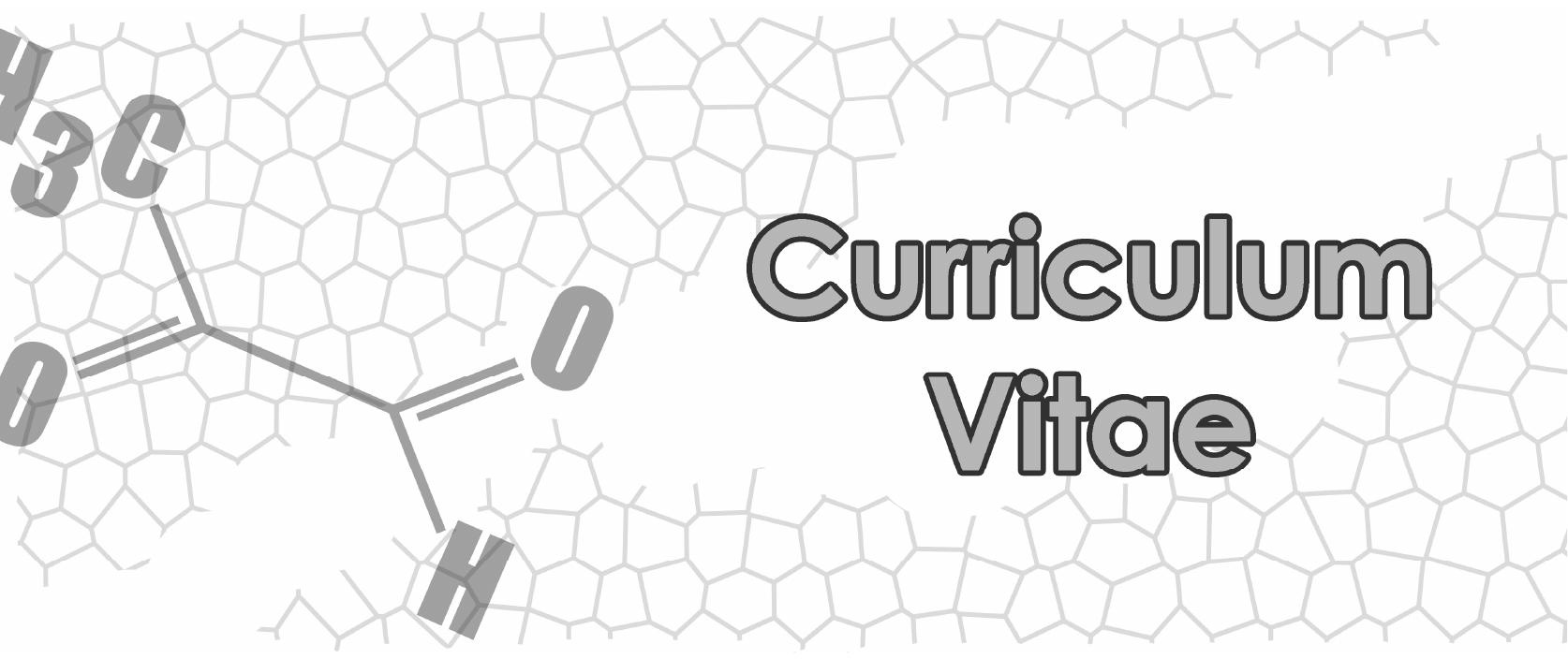


Olaf Brouwers werd geboren op 12 juni 1982 in het Atrium ziekenhuis te Heerlen. Hij groeide op in het kleine dorpje Ransdaal gelegen in het Limburgse heuvellandschap. In 1994 begon hij zijn middelbare schooltijd op het Bernardinuscollege te Heerlen, waaraan hij in 1999 zijn HAVO diploma behaalde. Zijn keuze voor het Hoger Laboratorium Onderzoek (HLO) als vervolgstudie kwam mede door zijn interesse in biologie en scheikunde niet als een verrassing. Tussen 1999 en 2003 doorliep hij het HLO en koos hij voor een biomedisch georiënteerde opleidingsrichting, die hij afsloot met een stage bij Klinische Genetica aan de Universiteit Maastricht. Hier werd zijn verlangen om ooit zelfstandig onderzoek uit te voeren vergroot en besloot hij om de Master Biomedische Wetenschappen op de Radboud Universiteit te Nijmegen te volgen. Na een academische (Departement of Cell Physiology) en farmaceutische (toenmalige Organon) stage kwam hij in 2006, met diploma op zak, weer terug naar Zuid-Limburg. Aan de Universiteit Maastricht begon hij toen zijn promotie onderzoek bij de vakgroep Interne Geneeskunde, waarvan de resultaten staan beschreven in dit proefschrift. 


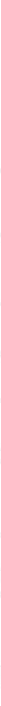


Brouwers O, Niessen P, Miyata T, Janssen B, De Mey J, Van Nieuwenhoven F, Stehouwer C, Schalkwijk C. Glyoxalase-I overexpression improves blood pressure independently of cardiac function.

Brouwers O, Niessen P, Miyata T, Brownlee M, De Mey J, Stehouwer C, Schalkwijk C. Glyoxalase-I overexpression improves endothelial dysfunction and attenuates renal impairment in diabetic rats.

Brouwers O, Niessen P, Ferreira I, Miyata T, Scheffer P, Teerlink T, Schrauwen P, Brownlee M, Stehouwer C, Schalkwijk C. Overexpression of glyoxalase-I reduces hyperglycaemiainduced levels of advanced glycation endproducts and oxidative stress in diabetic rats. $J$ Biol Chem. 2011 Jan; 286(2):1374-1380.

Brouwers O, Niessen P, Haenen G, Miyata T, Brownlee M, Stehouwer C, De Mey J, Schalkwijk C. Hyperglycaemia-induced impairment of endothelium-dependent vasorelaxation in rat mesenteric arteries is mediated by intracellular methylglyoxal levels in a pathway dependent on oxidative stress. Diabetologia. 2010 May; 53(5):989-1000.

Engelen L, Ferreira I, Brouwers O, Henry R, Dekker J, Nijpels G, Heine R, van Greevenbroek $M$, van der Kallen C, Blaak E, Feskens E, ten Cate $H$, Stehouwer C, Schalkwijk C. Polymorphisms in glyoxalase 1 gene are not associated with vascular complications: the Hoorn and CoDAM studies. J Hypertens. 2009 Jul;27(7):1399-1403.

Schalkwijk C, Brouwers O, Stehouwer C. Modulation of insulin action by advanced glycation endproducts: a new player in the field. Horm Metab Res. 2008 Sep;40(9):614619.

Brouwers O, Teerlink T, van Bezu J, Barto R, Stehouwer C, Schalkwijk C. Methylglyoxal and methylglyoxal-arginine adducts do not directly inhibit endothelial nitric oxide synthase. Ann N Y Acad Sci. 2008 Apr;1126:231-234. 


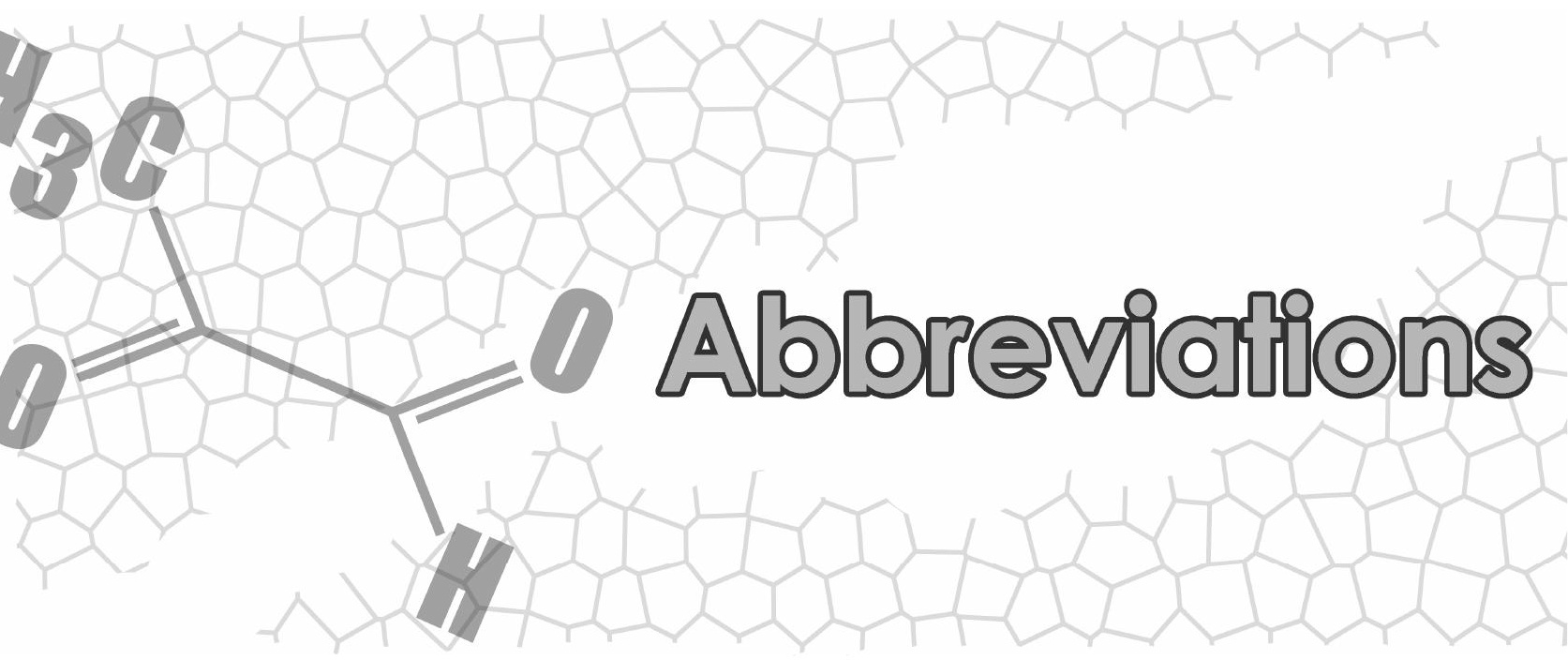


3-DG 3-deoxyglucosone

AD aldehyde dehydrogenase

ADMA asymmetric dimethylarginine

AG aminoguanidine

AGE advanced glycation endproduct

ALB albumin

ANP atrial natriuretic peptide

ANT-1 adenine nucleotide translocase-1

AP arginine-pyrimidine

Apo apolipoprotein

BNP brain natriuretic peptide

CEL $\quad \mathrm{N}_{\varepsilon}$-(1-carboxyethyl)lysine

CML $\quad \mathrm{N}_{\varepsilon}$-(1-carboxymethyl)lysine

CO cardiac output

Col1a1 collagen type I alpha 1

CTGF connective tissue growth factor

DAG diacylglycerol

DCFH-DA 5-carboxy-2',7'-dichlorodihydrofluorescin-diacetate

DHAP dihydroxyaceton phosphate

ECM extracellular matrix

EDHF endothelium-derived

hyperpolarizing factor

eNOS endothelial nitric oxide synthase

G3P glyceraldehyde-3-phosphate

GAPDH glyceraldehyde-3-phosphate

dehydrogenase

GO glyoxal

GSH reduced glutathione

GSSG oxidized glutathione

HIF hypoxia-induced factor

HNE hydroxynonenal

HSP hexosamine pathway

HUVECs human umbilical vein

endothelial cells

ICAM-1 intracellular adhesion molecule-1

iNOS inducible nitric oxide synthase

IP

KIM-1 kidney injury molecule-1

$\mathrm{KLH} \quad$ keyhole limpet hemocyanin

LDL low density lipoprotein

LIP lipocalin

LV left ventricle
MDA malondialdehyde

MG-H1 N $\mathrm{N}_{\delta}$-(5-hydro-5-methyl-4imidazolon-2-yl)-L-ornithine

MG-H2 2-amino-5-(2-amino-5-hydro-5methyl-4-imidazolon-1-yl)pentanoic acid

MG-H3 2-amino-5-(2-amino-4-hydro-4methyl-5-imidazolon-1-yl)pentanoic acid

MGO methylglyoxal

MMP matrix metalloproteinase

MODIC 2-ammonia-6-(\{2-[4-ammonio-5oxido-5-oxopentyl)amino]4methyl-4,5-dihydro- $1 \mathrm{H}$ imidazol-5ylidene\}ino)hexanoate

MOLD 1,3-di( $\mathrm{N}_{\varepsilon}$-lysino)-4-methylimidazolium

MTT 3-[4,5-dimethylthiazol-2-yl]-2,5diphenyl tetrazolium bromide

NADPH nicotinamide adenine dinucleotide phosphate

NO nitric oxide

NT nitrotyrosine

OST osteopontin

PAI-1 plasminogen activator inhibitor1

PARP poly(ADP-ribose) polymerases

PCA per chloric acid

PKC protein kinase $C$

RAGE receptor for $A G E$

ROS reactive oxygen species

SDMA symmetric dimethylarginine

SMC smooth muscle cell

SNP sodium nitroprusside

SOD super oxide dismutase

STZ streptozotocin

T1DM type 1 diabetes mellitus

T2DM type 2 diabetes mellitus

THP tetrahydropyrimidine

TNF $\alpha$ tumour necrose factor alpha

UCPs uncoupling proteins

VCAM-1 vascular adhesion molecule-1

VEGF vascular endothelial growth factor 


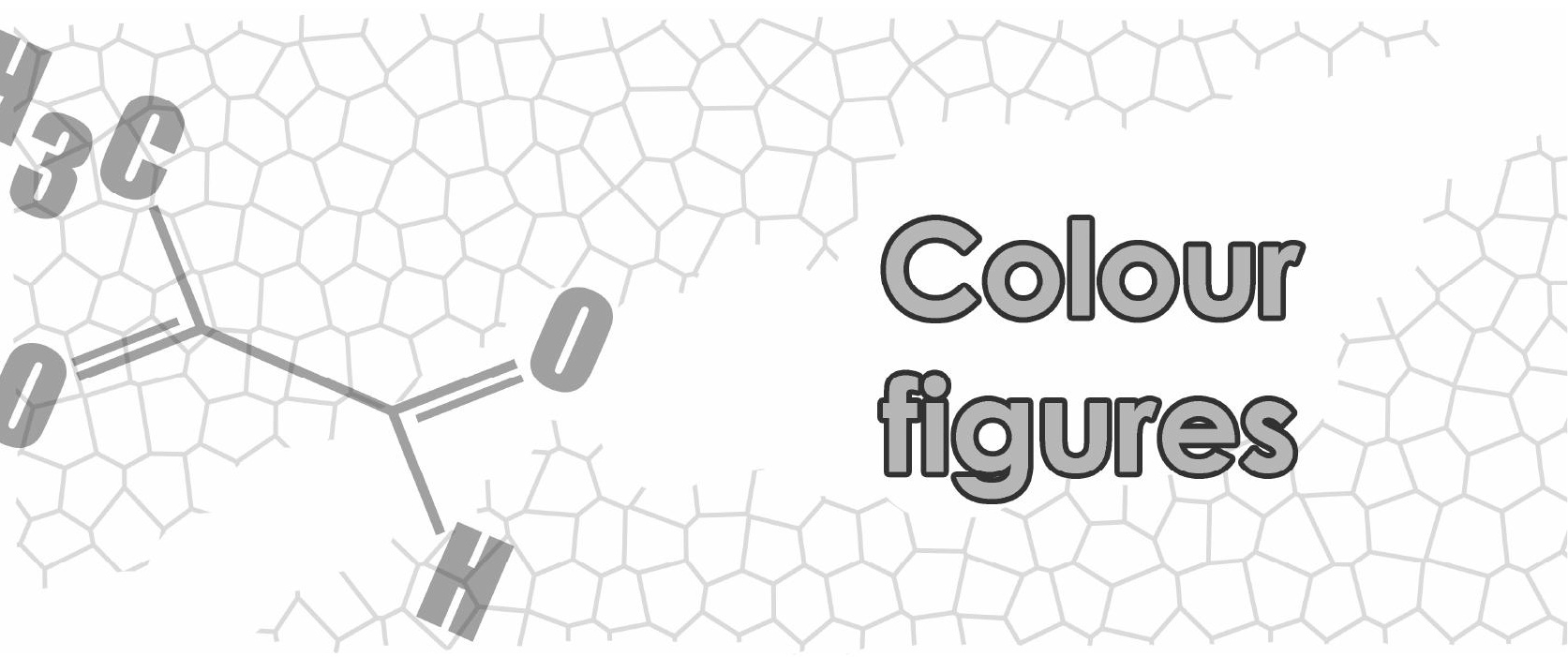




\section{Chapter 3}

3D
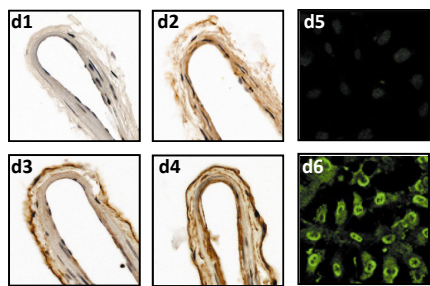

5A
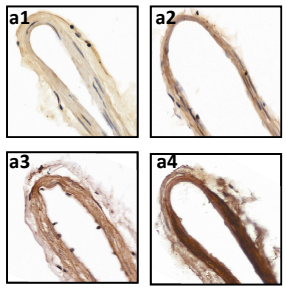

4A

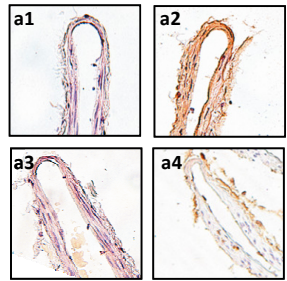

6B

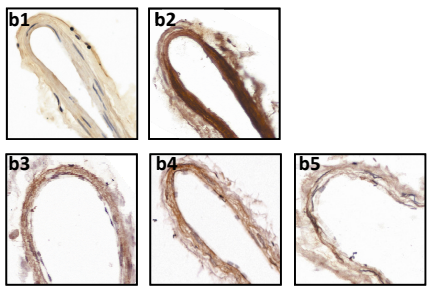

\section{Chapter 4}

1C
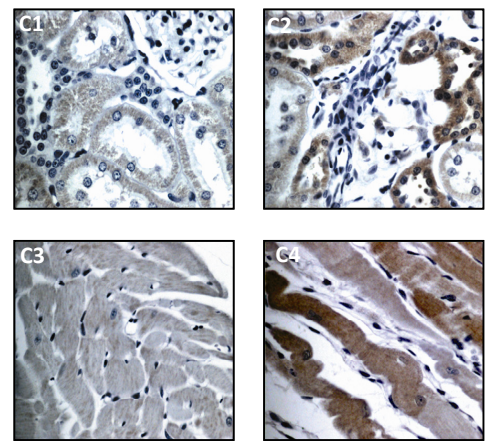


\section{Chapter 5}

1A
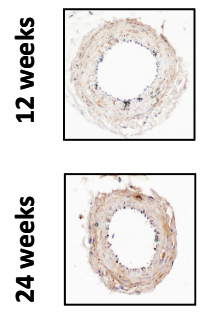

control
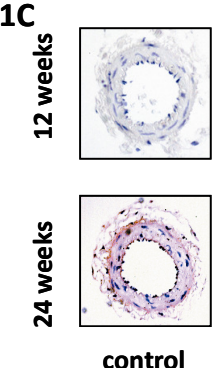
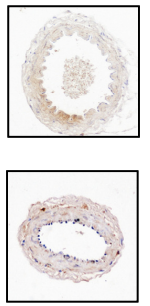

diabetic
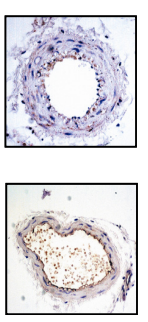

diabetic
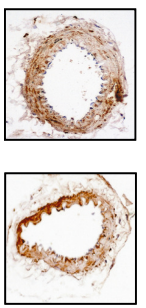

diabetic

GLO-I
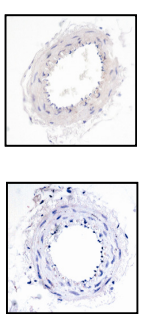

diabetic

GLO-I
1B
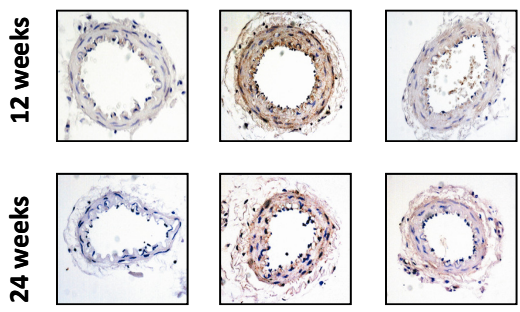

control

diabetic

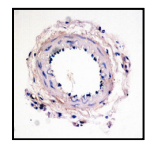

diabetic

GLO-I
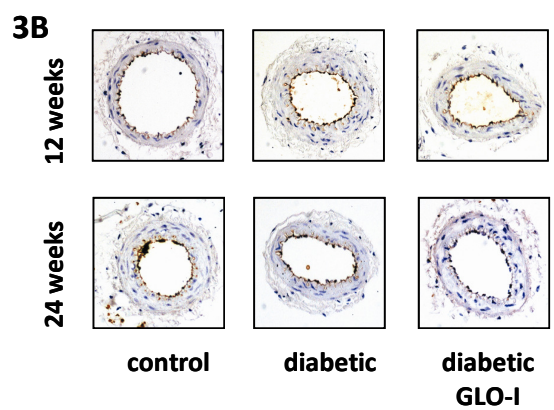

\section{Supplement Chapter 5}

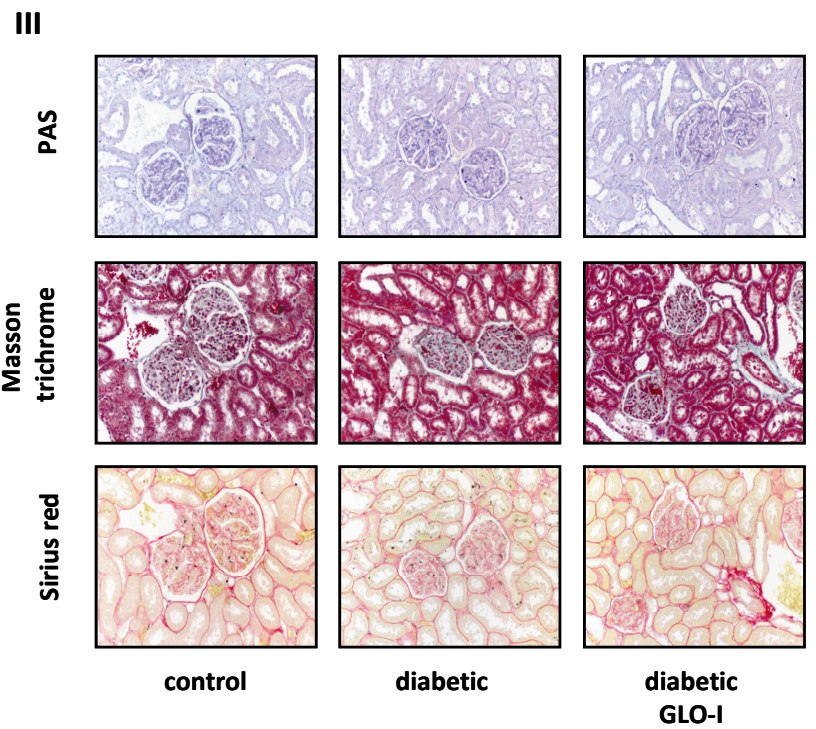


